Ricardo Shirota Filho

\title{
Processos de Decisão Markovianos com Probabilidades Imprecisas e Representações Relacionais: Algoritmos e Fundamentos
}

Tese apresentada a Escola Politécnica da Universidade de São Paulo para obtenção do Título de Doutor em Engenharia Mecânica. 
Ricardo Shirota Filho

\section{Processos de Decisão Markovianos com Probabilidades Imprecisas e Representações Relacionais: Algoritmos e Fundamentos}

Tese apresentada a Escola Politécnica da Universidade de São Paulo para obtenção do Título de Doutor em Engenharia Mecânica.

Área de concentração:

Controle e Automação Mecânica

Orientador:

Prof. Dr. Fabio Gagliardi Cozman 
Este exemplar foi revisado e alterado em relação à versão original, sob responsabilidade única do autor e com a anuência de seu orientador.

São Paulo, 29 de junho de 2012.

Assinatura do autor

Assinatura do orientador

FICHA CATALOGRÁFICA

Shirota Filho, Ricardo

Processos de decisão Markovianos com probabilidades imprecisas e representações relacionais: algoritmos e fundamentos / . -- ed.rev. -- São Paulo, 2012.

$148 \mathrm{p}$.

Tese (Doutorado) - Escola Politécnica da Universidade de São Paulo. Departamento de Engenharia Mecatrônica e de Sistemas Mecânicos.

1.Algoritmos 2.Cadeias de Markov 3.Probabilidade 4.Tomada de decisão I.Universidade de São Paulo. Escola Politécnica. Departamento de Engenharia Mecatrônica e de Sistemas Mecânicos II.t. 



\section{Agradecimentos}

Um projeto da dimensão de um doutorado dificilmente é um trabalho solitário, e apesar do esforço maior ser do próprio candidato, inúmeras colaborações, sugestões e discussões enriquecem o trabalho como um todo. Muitas vezes elas são fundamentais para correções de rumo, e as vezes, contribuem para novos (mas inspiradores) desvios. Não tenho a pretenção de lembrar cada colaboração individualmente, então àqueles que não se sentirem contemplados na listagem a seguir, fica, desde já, meus sinceros agradecimentos.

Primeiramente, gostaria de agradecer à minha família. A família é o começo de tudo, e seu apoio é fundamental em todos os momentos. Nas horas difíceis é a família que geralmente vem ao socorro, e vindo de uma casa onde a maioria optou pela carreira acadêmica, o apoio e a compreensão nunca falharam.

Professores, colegas, amigos, revisores, funcionários das mais diversas instâncias da universidade, também merecem seu reconhecimento. Cada um, a sua maneira, contribuiu para o desenvolvimento pessoal, profissional e acadêmico, e sabem o quanto foram importantes nessa caminhada.

Agradeço também a FAPESP, Fundação de Amparo à Pesquisa do Estado de São Paulo, que forneceu o suporte financeiro necessário para a realização desse projeto.

E por fim, gostaria de deixar a minha especial apreciação ao ilustríssimo Prof. Dr. Fabio G. Cozman, que apesar dos meus inúmeros desvios pelo caminho, nunca deixou de acreditar no meu potencial. 
"Valeu a pena? Tudo vale a pena Se a alma não é pequena." De Mar Português, Fernando Pessoa 


\section{Resumo}

Este trabalho é dedicado ao desenvolvimento teórico e algorítmico de processos de decisão markovianos com probabilidades imprecisas e representações relacionais. Na literatura, essa configuração tem sido importante dentro da área de planejamento em inteligência artificial, onde o uso de representações relacionais permite obter descrições compactas, e o emprego de probabilidades imprecisas resulta em formas mais gerais de incerteza. São três as principais contribuições deste trabalho. Primeiro, efetua-se uma discussão sobre os fundamentos de tomada de decisão sequencial com probabilidades imprecisas, em que evidencia-se alguns problemas ainda em aberto. Esses resultados afetam diretamente o (porém não restrito ao) modelo de interesse deste trabalho, os processos de decisão markovianos com probabilidades imprecisas. Segundo, propõe-se três algoritmos para processos de decisão markovianos com probabilidades imprecisas baseadas em programação (otimização) matemática. E terceiro, desenvolvem-se ideias propostas por Trevizan, Cozman e de Barros (2008) no uso de variantes do algoritmo Real-Time Dynamic Programming para resolução de problemas de planejamento probabilístico descritos através de versões estendidas da linguagem de descrição de domínios de planejamento (PPDDL).

Palavras-chave: Processo de decisão Markoviano. Probabilidades imprecisas. Representações relacionais. Algoritmos. Tomada de decisão sequencial. Fundamentos. 


\section{Abstract}

This work is devoted to the theoretical and algorithmic development of Markov Decision Processes with Imprecise Probabilities and relational representations. In the literature, this configuration is important within artificial intelligence planning, where the use of relational representations allow compact representations and imprecise probabilities result in a more general form of uncertainty. There are three main contributions. First, we present a brief discussion of the foundations of decision making with imprecise probabilities, pointing towards key questions that remain unanswered. These results have direct influence upon the model discussed within this text, that is, Markov Decision Processes with Imprecise Probabilities. Second, we propose three algorithms for Markov Decision Processes with Imprecise Probabilities based on mathematical programming. And third, we develop ideas proposed by Trevizan, Cozman e de Barros (2008) on the use of variants of Real-Time Dynamic Programming to solve problems of probabilistic planning described by an extension of the Probabilistic Planning Domain Definition Language (PPDDL).

Keywords: Markov Decision Process. Imprecise probabilities. Relational representations. Algorithm. Sequential decision making. Foundations. 


\section{Sumário}

Lista de Abreviaturas

1 Introdução

2 Processo de Decisão Markoviano

2.1 Formulação Matemática . . . . . . . . . . . . . . . . . . . . . . . . 19

2.2 Critérios de Otimalidade . . . . . . . . . . . . . . . . . 20

2.3 Algoritmos . . . . . . . . . . . . . . . . . . . . . 22

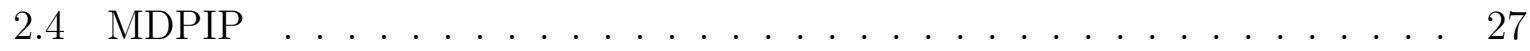

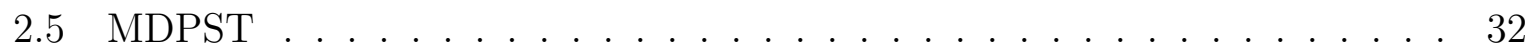

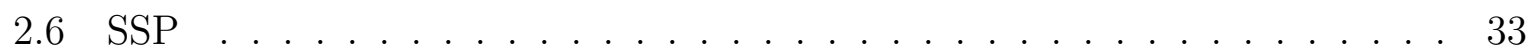

3 Fundamentos $\quad 35$

4 Planejamento $\quad 44$

4.1 Caracterização de Domínios . . . . . . . . . . . . . . 45

4.2 Planejamento Clássico . . . . . . . . . . . . . . . . . . . 50

4.3 Planejamento sob Incerteza $\ldots \ldots \ldots$. . . . . . . . . . . . . 51

4.3.1 Planejamento Não-determinístico . . . . . . . . . . . . . . . 52

4.3.2 Planejamento Probabilístico . . . . . . . . . . . . . 53 
4.4 Representação Enumerativa vs Fatorada . . . . . . . . . . . . . . . . . . . 54

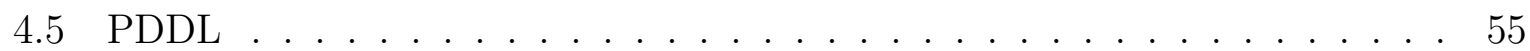

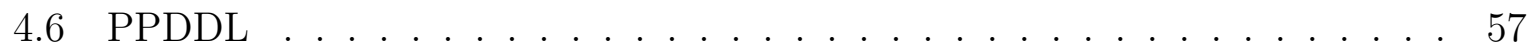

4.7 PDL1 e PDL2 . . . . . . . . . . . . . . . . . . 57

4.8 PDL3 f . . . . . . . . . . . . . . . . . . . . . . . . 59

5 Programação Matemática para MDPIP $\quad 61$

5.1 Programação Binível . . . . . . . . . . . . . . . . . . 62

5.2 Programação Bilinear . . . . . . . . . . . . . . . . . . . . 63

5.3 Programação Inteira . . . . . . . . . . . . . . . . . . . 64

6 RTDP com Probabilidades Imprecisas $\quad 67$

6.1 Algoritmo RTPD Modificado . . . . . . . . . . . . . . . . 69

$\begin{array}{lll}7 & \text { Trabalhos Futuros } & 73\end{array}$

8 Conclusões $\quad 76$

$\begin{array}{ll}\text { Referências Bibliográficas } & 78\end{array}$

$\begin{array}{ll}\text { Apêndices } & 85\end{array}$

I Artigo: ISIPTA $2007 \quad 85$

II Artigo: Sequential Decision Processes under Act-State Independence with Arbitrary Choice Functions (Versão completa) 96 


\section{Lista de Abreviaturas}

ADL Action Definition Language

AIPS Artificial Intelligence Planning Systems

AMDP Algebraic Markov Decision Process

BMDP Bounded-parameter Markov Decision Process

EBNF Extendend Backus-Naur Form

IA Inteligência Artificial

IPC International Planning Competition

LRTA* Learning Real-Time $A^{*}$

LRTDP Labeled Real-Time Dynamic Programming

MDP Markov Decision Process

MDPIP Markov Decision Process with Imprecise Probabilities

MDPST Markov Decision Process with Set-valued Transitions

PDDL Planning Domain Definition Language

PDL Planning Definition Language

PPDDL Probabilistic Planning Domain Definition Language

RDDL Relational Dynamic Influence Diagram Language

RTA* Real-Time $A^{*}$ 
RTDP Real-Time Dynamic Programming

SSP Stochastic Shortest Path

STRIPS Stanford Research Institute Problem Solver

UCPOP Universal Conditional Partial Order Planner

UMCP Universal Method-Composition Planner 
"Quase tudo que um ser humano faz envolve decisões. Portanto, teorizar sobre decisões é quase igual a teorizar sobre as atividades humanas." 


\section{Introdução}

Tomada de decisão sequencial é um paradigma importante dentro da ciência. O seguinte parágrafo, da autoria de Harmanec (2002), ilustra muito bem como ele pode ser aplicada a problemas do mundo real:

Todos os dias, enfrentamos a necessidade de tomar decisões que dependem das nossas ações passadas e que irão influenciar nosso bem-estar e as opções disponíveis no futuro. Temos que decidir entre dirigir para o trabalho ou utilizar transporte público; um médico precisa decidir qual tratamento indicar para um paciente; e assim por diante. Para tomar boas decisões em situações como essas, não podemos considerá-las em isolamento, mas no contexto de decisões passadas e futuras. Por exemplo, se eu gastar todo o meu dinheiro hoje, perco a opção de comprar qualquer alimento no dia de amanhã.

Decisões são eventos importantes na vida do ser humano. Na história, existem diversos exemplos de indivíduos que ficaram marcados pelas escolhas que fizeram, ou até mesmo por aquelas que deixaram de fazer - que, no final, nada mais é do que a decisão de não escolher. Durante a nossa existência, somos apresentados a inúmeras situações que requerem uma posição sobre um problema delicado, cujas consequências podem se estender pelo resto da vida. Errar, nesses casos, pode não ser uma alternativa viável.

A dificuldade em fazer escolhas também está, muitas vezes, relacionada à existência de incertezas. Incertezas são partes integrantes do mundo, e surgem pelos mais diversos motivos: ambiguidade, ignorância, preguiça, etc. Por sua causa, é comum não ter como 
antecipar plenamente as consequências de uma escolha, que acaba por dificultar a comparação entre alternativas e a eleição de uma opção superior. Nesses casos, geralmente vê-se obrigado a utilizar alguma técnica (que, em certas ocasiões, não deixa de ser o bom e velho "chute") para decidir qual estratégia é mais adequada. Fica nítido, também, que um melhor entendimento do problema pode contribuir muito para a realização de uma escolha melhor, o que naturalmente levaria a um desempenho superior.

Decisões, no entanto, não consistem apenas das escolhas difíceis. Atitudes menores, cotidianas, também são consequências de nossas decisões. A roupa que se veste, a comida que se come, o horário que se acorda, são todos exemplos de decisões. Pode-se associar uma decisão a praticamente tudo que se faz, pois a ação pode ser vista como a consequência da decisão por fazê-la (algumas exceções seriam, por exemplo, atitudes involuntárias como o batimento do coração). Dessa forma, entender como essas escolhas são feitas poderia permitir simular, prever ou até mesmo substituir o ser humano em certas tarefas, liberando-o de atividades trabalhosas e permitindo que ele se envolva em tarefas mais importantes.

A investigação da razão humana no contexto das decisões está contido no campo da ciência conhecida como teoria da decisão. Talvez mais do que um mero campo da ciência, teoria da decisão pode ser vista como um tema de estudo que agrega pesquisadores das mais diversas especialidades. Conceitos relevantes da psicologia, filosofia, matemática, economia e engenharia, só para citar algumas, são fundamentais para a construção das teorias hoje vigentes, cuja diversidade de idéias fornece um rico campo para ser explorado.

A teoria da decisão possui dois paradigmas. O primeiro, conhecido como teoria descritiva ou comportamental, estuda o comportamento humano e a sua forma (muitas vezes imperfeita) de realizar escolhas. Aqui, o objetivo é obter uma descrição da razão humana, de forma a compreender melhor como os humanos se comportam. Isso é importante, por exemplo, para tentar antecipar mudanças comportamentais que possam ter grandes impactos (sejam econômicos, na saúde, ou outros) em um grupo de pessoas, informação que pode ser usado por empresas (e.g. para prever como mudanças de preço interferem na 
demanda de um produto) ou governos (e.g. para propor políticas públicas antecipando um problema iminente ou realizar interferências econômicas preventivas evitando catástrofes maiores).

A segunda linha é conhecida como teoria normativa. Na teoria normativa, visa-se obter um conjunto de normas regendo as ações de um agente, que levam a um comportamento ótimo (ou seja, realizar as melhores escolhas possíveis). Como ela prescreve um comportamento - que é determinado pelas normas - também é conhecida como teoria prescritiva. Em geral, o intuito é ajudar o ser humano a realizar decisões melhores, ou ainda, apresentando uma alternativa viável em situações em que um ser humano não teria condições de avaliar todas as possibilidades disponíveis. Técnicas derivadas dessa abordagem são atualmente predominantes para lidar com tomada de decisão sequencial sob incerteza, e muitas soluções tecnológicas são hoje possíveis graças à aplicação de seus modelos.

Planejamento, ou seja, a ciência da construção de planos, é um problema central em inteligência artificial. Partindo de uma situação inicial pré-determinada, o problema consiste em obter um plano de ação que leve o agente até uma situação final desejada. Diversos fatores influenciam na dificuldade em elaborar esse plano, além da forma do plano em si. Por exemplo, nos casos em que as incertezas do problema não são significativos (isto é, a dinâmica do sistema é bem definida e conhecida), um plano pode ser dado por uma sequência de ações, enquanto que nos casos em que as incertezas são significativas, tornase necessário utilizar uma política (ou seja, um mapa de estados para ações, especificando uma ação para cada estado possível do sistema).

O problema de planejamento é essencialmente um problema de decisão sequencial, e atualmente o modelo mais importante em planejamento é derivado de um procedimento para escolha sequencial, chamado de processo de decisão markoviano. A adaptação deste modelo para a realidade de planejamento exigiu, no entanto, diversas modificações na formulação original para adequar a ferramenta para os requisitos da área. Neste trabalho, investiga-se o emprego de duas modificações: 
1. o uso de representações fatoradas ou relacionais para estados e ações e

2. probabilidades imprecisas para descrever as incertezas.

O modelo base utiliza uma representação de estados e ações com transições probabilísticas para descrever o problema de decisão sequencial de interesse. A solução do problema consiste em uma política, que é um mapa de ações para estados, ou seja, uma atribuição de uma ação a ser executada em cada estado possível. Quando as ações especificadas pela política determinam um comportamento ótimo, que é avaliado através de uma função designada, a política é dita ótima. Em geral, é essa política que se deseja quando se resolve um processo de decisão markoviano.

Existem dois grandes problemas com essa formulação. Primeiro, o número de atribuições de probabilidades é exponencial com o tamanho do espaço de estados e ações. A tarefa de especificar cada um desses valores logo torna-se inviável, seja pela quantidade enorme de valores necessários, seja pela falta de dados ou de conhecimento sobre o processo para determinar tais valores. Segundo, a necessidade dos algoritmos clássicos em percorrer a totalidade do espaço de possibilidades torna a solução de problemas computacionalmente muito custoso mesmo para problemas médios, dificultando sua utilização em problemas mais complexos ou em casos com fortes requisitos de desempenho.

As contribuições deste trabalho seguem duas linhas. Na primeira, apresenta-se uma breve análise dos fundamentos de tomada de decisão sequencial com probabilidades imprecisas, em que são ressaltados alguns aspectos da teoria que merecem investigações mais profundas. Em grande parte, essas constatações são fruto do estágio realizado na Universidade de Durham, sob tutela do Dr. Matthias C. M Troffaes. Em um segundo momento, desenvolvem-se algoritmos para processos de decisão markovianos com probabilidades imprecisas visando sua utilização em problemas de planejamento, em que são aproveitadas algumas idéias desenvolvidas por Trevizan, Cozman e de Barros (2008).

O texto é estruturado da seguinte forma. O modelo básico é apresentado no Capítulo 2. No capítulo seguinte, apresenta-se a discussão sobre os fundamentos de decisões 
sequenciais com probabilidades imprecisas, baseada no trabalho realizado em Durham. O Capítulo 4 introduz o problema de planejamento. O Capítulo 5 contém três algoritmos baseados em programação matemática para MDPIP. No capítulo seguinte apresenta-se os resultados necessários para estender o algoritmo RTDP para probabilidades imprecisas. Por fim, apresentam-se algumas sugestões de trabalhos futuros e as conclusões deste trabalho. 


\section{Processo de Decisão Markoviano}

Processo de decisão markoviano (MDP, do inglês Markov Decision Processes), é um modelo matemático de estados e ações com transições estocásticas para problemas de decisão sequencial sob incerteza (WHITE, 1993; PUTERMAN, 1994; RUSSELL; NORVIG, 2010). Também chamado de programa dinâmico estocástico, problema de controle estocástico ou cadeia de Markov controlada (PUTERMAN, 1994), foi desenvolvido por Richard Bellman dentro da pesquisa operacional como sub-produto da investigação de programação dinâmica (BELLMAN, 2003, 1957). Sua flexibilidade permitiu a propagação para os mais diversos campos da ciência, tendo aplicações em áreas como engenharia, medicina, ecologia, economia e mais recentemente, inteligência artificial (WHITE, 1985, 1988; PUTERMAN, 1994; RUSSELL; NORVIG, 2010). Atualmente, é tido como o modelo de facto padrão para problemas de planejamento probabilístico (BOUTILIER; REITER; PRICE, 2001), e muitos dos progressos recentes derivam de pesquisas dessa área. Puterman (1994, Capítulo 2, p. 17) descreve o problema da seguinte forma:

Um tomador de decisão, agente, ou controlador é apresentado a um problema, ou como diriam alguns, uma oportunidade, de influenciar o comportamento de um sistema probabilístico que evolui no tempo. Isso é feito através da tomada de decisão ou escolha de ações. Seu objetivo é escolher uma sequência de ações que levam o sistema a um desempenho ótimo com relação a um critério de desempenho predeterminado. Como o sistema modelado é progressivo, o estado do sistema prévio à decisão de amanhã depende da decisão de hoje. Consequentemente, $[\ldots]$ decisões devem antecipar oportunidades e custos (ou 
recompensas) associados aos estados futuros do sistema.

Nesse momento, convém introduzir a formulação matemática utilizada no restante do capítulo. Mais adiante, abordam-se os diferentes critérios para avaliar soluções, e os algoritmos que permitem a obtenção de resultados ótimos. Logo a seguir, apresenta-se uma generalização do modelo, chamada de MDPIP, que relaxa a suposição de valores precisos de probabilidades. Como será visto, essa generalização possui algumas vantagens em comparação ao modelo tradicional. Apresenta-se, ainda, uma subclasse de MDPIPs que permite a unificação de problemas de planejamento probabilístico e não-determinístico.

\subsection{Formulação Matemática}

Um MDP consiste em uma tupla $\langle\mathcal{T}, \mathcal{S}, \mathcal{A}, P, C\rangle$, em que (PUTERMAn, 1994):

- $\mathcal{T}$ é um conjunto finito ou infinito de estágios de decisão. Em cada estágio, é esperado que o agente escolha uma ação a ser executada. Quando finito, diz-se que o problema é de horizonte finito. Quando infinito, diz-se de horizonte infinito.

- $\mathcal{S}$ é o conjunto de estados do sistema. Cada estado representa uma configuração específica de interesse, e em cada estágio o sistema encontra-se em um desses estados. Pode ser um conjunto finito ou infinito (PUTERMAn, 1994). Por simplificação, neste trabalho consideram-se conjuntos finitos.

- $\mathcal{A}$ é o conjunto de ações disponíveis ao agente. Em determinados casos, pode ser interessante estabelecer um conjunto $A(s)$ de ações para cada estado $s \in \mathcal{S}$. Novamente, consideram-se conjuntos finitos.

- $P$ é uma medida probabilística sobre os possíveis estados futuros. A notação utilizada é $P(r \mid s, a)$, indicando a probabilidade de transição para o estado $r$, partindo do estado $s$ e aplicando a ação $a$. Assume-se que $P(r \mid s, a) \geq 0$, para todo $r, s \in \mathcal{S}$ e $a \in A(s)$, e $\sum_{r \in \mathcal{S}} P(r \mid s, a)=1$. 
- $C$ é uma função que devolve o custo incorrido pelo agente. Pode depender do estado $(C(s))$, da ação $(C(a))$ ou ambos $(C(s, a))$. Recompensas são custos negativos, e são denotadas por $R$.

O histórico $H_{t}$ em um estágio $t$ é uma sequência de estados e ações visitadas pelo processo $\left[s_{0}, a_{0}, \ldots, a_{t-1}, s_{t}\right]$. Denota-se essa expressão recursivamente por $H_{t}=\left[H_{t-1}, a_{t-1}, s_{t}\right]$. A hipótese de Markov (de onde o nome do modelo é derivado (PUTERMAN, 1994)), é adotada no modelo, e determina que $P\left(s_{t} \mid H_{t-1}, a_{t}\right)=P\left(s_{t} \mid s_{t-1}, a_{t}\right)$, e consequentemente,

$$
P\left(h_{t}\right)=P\left(s_{t} \mid s_{t-1}, a_{t-1}\right) P\left(s_{t-1} \mid s_{t-2}, a_{t-2}\right) \ldots P\left(s_{1} \mid s_{0}, a_{0}\right) .
$$

Uma regra de decisão $d_{t}(s)$ indica a ação que deverá ser tomada num estado $s$ em um estágio t. Uma política $\pi$ é uma sequência de regras de decisões, uma para cada estágio. Uma política pode ser determinística ou aleatória, isto é, pode indicar ações com certeza, ou pode apenas indicar distribuições de probabilidades sobre as ações. Uma política pode ser ainda dependente ou não do histórico, isto é, pode depender de todos os estados e ações visitados em instantes anteriores ou apenas no estado atual. Uma política que não é dependente do histórico é chamada de markoviana. Uma política markoviana induz uma distribuição de probabilidades sobre históricos através da Expressão (2.1). A seleção de políticas depende do critério de avaliação que é utilizado para calcular o valor de uma política.

\subsection{Critérios de Otimalidade}

Para cada estado e estágio, um política indica qual ação o agente deve executar. Ao executar essa política, o agente recebe as recompensas (ou incorre os custos), que podem ser utilizadas para avaliar seu desempenho. A política com o melhor desempenho é chamada de política ótima, e é a solução para o MDP associado. A avaliação desse desempenho é feita através da função valor ou utilidade, que é determinada segundo um dos três critérios de otimalidade (PUTERMAN, 1994) explicados a seguir. 
Custo Total Esperado O critério do custo total esperado (Eq. (2.2)) pode ser visto como a extensão probabilística para a simples soma dos custos recebidos durante todo o processo. Dessa forma, a utilidade (ou valor) de uma política é computada através da soma dos valores esperados dos custos recebidas ao aplicar a política $\pi$. Quando o problema é de horizonte finito, obtém-se uma soma finita, cujo resultado é limitado desde que cada parcela também o seja. Porém, quando o problema é de horizonte infinito, a soma pode não ser limitada e torna-se necessário tomar outras precauções para que o problema seja bem formulado (BARTO; BRADTKE; SINGH, 1995). A expressão que representa esse critério é:

$$
v_{R T E}^{\pi}(s)=\lim _{N \rightarrow \infty} E_{s}^{\pi}\left\{\sum_{t=1}^{N} C\left(s_{t}, a_{t}\right)\right\},
$$

em que $E$ denota a esperança matemática.

Custo Médio Esperado O segundo critério é chamado de custo médio esperado, pois avalia a política através da média sobre os valores esperados dos custos. Esse critério (Eq. 2.3) é bastante utilizado em problemas nas quais decisões são feitas com grande frequência (PUterman, 1994). Por esse motivo, é bastante utilizado na área de telecomunicações (ALTMAN, 1999):

$$
v_{R M E}^{\pi}(s)=\lim _{N \rightarrow \infty} \frac{1}{N} E_{s}^{\pi}\left\{\sum_{t=1}^{N} C\left(s_{t}, a_{t}\right)\right\} .
$$

Custo Total Descontado Esperado O último critério, custo total descontado esperado (ou apenas custo descontado esperado), é provavelmente o critério mais popular em MDP, e será adotado neste trabalho. Esse critério faz uso de um fator de desconto $\gamma, \gamma \in[0,1]$, que pondera o efeito de transições futuras sobre o valor da utilidade do estado. Note que se $\gamma=1$, a expressão se reduz ao critério de custo total esperado. Uma interessante interpretação econômica é associada a esse critério, em que $\gamma$ é visto como um indicador da aversão (preferência) do agente por custos (recompensas) a curto ou longo prazo. Valores de $\gamma$ menores (mais próximos de zero), indicam que o agente tem preferência pelo presente, e não se importa com o futuro. Por outro lado, valores maiores 
de $\gamma$ indicam que o agente se preocupa também com o futuro, e estaria disposto a abrir mão de ganhos no presente se isso implicar em ganhos maiores no futuro. A expressão fica da seguinte forma:

$$
v_{R T D E}^{\pi}(s)=\lim _{N \rightarrow \infty} E_{s}^{\pi}\left\{\sum_{t=1}^{N} \gamma^{t-1} C\left(s_{t}, a_{t}\right)\right\} .
$$

O uso de um critério de avaliação associado a um processo de otimização origina a Equação de Otimalidade, mais conhecida como Equação de Bellman. Essa equação é de fundamental importância em MDP, e todos os algoritmos derivam dela de alguma forma. Para o critério de recompensas descontadas esperadas, a Equação de Bellman é:

$$
V^{*}(s)=\min _{a \in A(s)}\left(C(s, a)+\gamma \sum_{r \in \mathcal{S}} P(r \mid s, a) V^{*}(r)\right) .
$$

\subsection{Algoritmos}

Tradicionalmente, três algoritmos são utilizados para obtenção de políticas ótimas em MDPs. A seguir, descreve-se cada uma delas, sem entrar em muitos detalhes. Uma discussão mais profunda pode ser encontrada em Puterman (1994). Ao final, o algoritmo RTDP é apresentado, que será utilizado mais para frente em conexão com planejamento.

Iteração de Valor A aplicação direta de programação dinâmica ou indução regressiva ao contexto de MDP é chamada de iteração de valor. Esse algoritmo baseia-se na aplicação recursiva da equação de Bellman (Eq. (2.5)), o que resulta em um mapa de contrações na função valor (BELLMAN, 2003; DENARDO, 1967; PUTERMAN, 1994; RUSSELL; NORVIG, 2010). Isso significa que a cada nova aplicação da equação de Bellman, o comportamento do erro $\epsilon$ da aproximação $V_{n+1}$ é monotônico decrescente, ou seja, o erro sempre diminui. O algoritmo pode ser descrito da seguinte forma (extraído de Puterman (1994, p. 161)):

1. Selecione (arbitrariamente) $V_{0}$, especifique $\epsilon>0$ e estabeleça $n=0$. 
2. Para cada $s \in \mathcal{S}$, calcule $V_{n+1}(s)$ através de:

$$
V_{n+1}(s)=\min _{a \in A(s)}\left(C(s, a)+\sum_{r \in \mathcal{S}} \gamma P(r \mid s, a) V_{n}(r)\right) .
$$

3. (Critério de parada) Se

$$
\left\|V_{n+1}(s)-V_{n}(s)\right\|<\frac{\epsilon(1-\gamma)}{2 \gamma}
$$

para todos $s \in \mathcal{S}$, vá para o passo 4. Caso contrário, incremente $n$ uma unidade e retorne ao passo 2 .

4. Para cada $s \in \mathcal{S}$, escolha

$$
d_{\epsilon}(s) \in \underset{a \in A(s)}{\operatorname{argmin}}\left(C(s, a)+\sum_{r \in \mathcal{S}} \gamma P(r \mid s, a) V_{n}(r)\right)
$$

e pare.

A política ótima consiste na regra de decisão $d_{\epsilon}(s)$ aplicada em todo estágio.

Formas mais eficientes dos algoritmos são obtidos ao utilizar os novos valores de utilidade assim que eles se tornam disponíveis. Por exemplo, se o valor de $V_{n+1}\left(s_{1}\right)$ já tiver sido calculado, ele pode ser subsituído no lugar de $V_{n}\left(s_{1}\right)$ nos cálculos subsequentes. Formas assíncronas desse algoritmo também foram desenvolvidas, em que a atualização de certos estados ocorre com mais frequência que em outros (BERTSEKAS, 1982), como será explicado adiante.

Iteração de Políticas Enquanto o algoritmo de iteração de valor procura encontrar pontos fixos, o algoritmo de iteração de políticas ou aproximações no espaço de políticas está mais diretamente relacionado à estrutura particular de MDPs (PUTERMAN, 1994). O algoritmo é dividido em duas partes. Na primeira, chamada de avaliação da política, utiliza-se uma política escolhida para atualizar os valores dos estados. A segunda fase, aperfeiçoamento da política, emprega essas utilidades atualizadas para tentar encontrar uma política que tenha desempenho melhor. Se for possível encontrar uma política supe- 
rior, ela é usada para recalcular os valores dos estados e o processo se repete. Se nenhuma política exibir melhor desempenho, a política ótima foi encontrada e o algoritmo termina. O pseudo-código é apresentado a seguir (extraído de Puterman (1994)).

1. Estabeleça $n=0$, e selecione uma regra de decisão arbitrária $d_{0} \in D$.

2. (Avaliação da política) Obtenha $V_{n}$, através do cálculo

$$
\left(I-\gamma P_{d_{n}}\right) v=c_{d_{n}}
$$

3. (Aperfeiçoamento da política) Escolha $d_{n+1}$ para satisfazer

$$
d_{n+1} \in \underset{d \in D}{\operatorname{argmin}}\left(c_{d}+\gamma P_{d} v^{n}\right)
$$

estabelecendo $d_{n+1}=d_{n}$ se possível.

4. Se $d_{n+1}=d_{n}$, pare e estabeleça $d^{*}=d_{n}$. Caso contrário incremente $\mathrm{n}$ e retorne ao passo 2 .

Uma das vantagens desse algoritmo em comparação ao algoritmo de iteração de valor é que ele devolve explicitamente uma política, enquanto a iteração de valor devolve apenas os valores das utilidades dos estados (a política está implícita e precisa ser extraída da utilidade dos estados). Porém, ele mostra-se ineficiente e não é recomendado para problemas de horizonte finito (PUTERMAN, 1994, Seção 6.4, p. 174)

Programação Linear O terceiro algoritmo clássico para MDP é baseado em programação linear, que possui a seguinte forma:

$$
\begin{array}{ll}
\underset{V}{\operatorname{minimizar}} & \sum_{s} V(s) \\
\text { sujeito a } & V(s) \leq C(s, a)+\gamma \sum_{r} P(r \mid s, a) V(r) \quad \forall s, r \in \mathcal{S}, a \in A(s) .
\end{array}
$$

Essa formulação foi inicialmente proposta em D'Epenoux (1963), mas é considerada uma alternativa ineficiente em comparação aos outros dois métodos (PUTERMAN, 1994). Po- 
rém, algumas razões foram apontadas na literatura surgerindo que ela é importante em algumas situações. Primeiro, programação linear fornece uma maneira de lidar com MDPs com restrições (Constrained MDP) de uma forma bastante natural (FEINBERG; SHWARTZ, 2002). Inclusive, durante muito tempo programação linear foi a única forma de lidar com esse tipo de problema (PUTERMAn, 1994; ALTMAN, 1994). Segundo, diversos algoritmos baseados na aproximação da função valor por funções de menor dimensão são baseados em programação linear (SCHWEITZER; SEIDMANN, 1985; GUESTRIN et al., 2003). Programação linear também é interessante devido a facilidade em se realizar análise de sensibilidade (PUTERMAN, 1994), e essa abordagem mostra-se válida em contextos bastante gerais (FEINBERG; SHWARTZ, 2002). Segundo Hordijk e Kallenberg (1984), existem problemas em que a abordagem de programação dinâmica não é possível, e soluções podem ser obtidas apenas através de programação linear. Por fim, programação linear permite obter soluções exatas sem a necessidade de especificar um critério de parada (condições necessárias para os algoritmos de iteração de valor e de políticas), que é particularmente importante para avaliar o desempenho de algoritmos.

Mais recentemente, técnicas baseadas em programação linear foram utilizadas com sucesso para resolver uma forma do modelo chamado de MDP fatorado (e.g. Guestrin et al. (2003)), que serão apresentados adiante.

RTDP RTDP (Real-Time Dynamic Programming) é um algoritmo baseado em programação dinâmica que permite a melhora do desempenho com a experiência (BARTO; BRADTKE; SINGH, 1995). O algoritmo foi resultado da constatação que o algoritmo Learning Real-Time A* (LRTA*) (KORF, 1990) é parecido com uma forma de programação dinâmica chamada de programação dinâmica assíncrona (BARTO; BRADTKE; SINGH, 1995). RTDP é a generalização do LRTA* para problemas envolvendo incertezas (BARTO; BRADTKE; SINGH, 1995). LRTA*, por sua vez, é derivado do algoritmo A*, um algoritmo bastante conhecido para realizar busca em profundidade (KORF, 1990; RUSSELL; NORVIG, 2010), usado com bastante sucesso em problemas de busca heurística de agente simples 
(KORF, 1990). Duas desvantagens de $\mathrm{A}^{*}$ e suas variantes (e.g. Iterative Deepening A*) são que na prática elas exigem tempo exponencial de execução, e precisam realizar a busca até que a solução completa seja encontrada antes de escolher qualquer ação (o algoritmo precisa rodar até obter a solução completa para o problema antes de escolher até mesmo a primeira ação). Korf 1987, 1988, 1990 introduziu duas variações de A*. Real-Time A* (RTA*) é uma modificação de $A^{*}$ que permite o algoritmo escolher uma ação sem ter que realizar a busca completa no espaço, reduzindo drasticamente o tempo necessário para obter uma solução. Learning Real-Time A* (LRTA*) mantém as vantagens do RTA* e explora o benefécio de se aprender uma heurística durante repetidas tentativas de se resolver o problema. Ao notar que LRTA* possui fortes conexões com programação dinâmica assíncrona, Barto, Bradtke e Singh (1995) sugeriu modificações ao algoritmo original que permitiu sua aplicação em problemas estocásticos. Esse novo algoritmo foi chamado de RTDP.

O RTDP funciona da seguinte maneira. Considere $h$ uma função de avaliação dos estados (ou seja, uma heurística). O Q-value de uma ação $a$ em um estado $s$ pode ser calculado por:

$$
Q(s, a)=C(s, a)+\gamma \sum_{r \in \mathcal{S}} P(r \mid s, a) h(r) .
$$

Esse $Q$-value é usado para fazer uma atualização gulosa da estimativa $h_{t}$ da função ótima $f^{*}$ após o passo de computação do estágio t do programa dinâmico assíncrono. Dessa forma,

$$
h_{t+1}=\max _{a \in \mathcal{A}}\left[C(s, a)+\sum_{r \in \mathcal{S}} P(r \mid s, a) h_{t}(r)\right] .
$$

A convergência do programa dinâmico assíncrono e do RTDP é garantido desde que quatro condições sejam satisfeitas (BARTO; BRADTKE; SINGH, 1995):

- o custo inicial de cada estado meta é zero,

- existe pelo menos uma política própria ${ }^{1}$,

\footnotetext{
${ }^{1}$ Uma política própria é uma política que implica em uma probabilidade não-nula de eventualmente alcançar um dos estados que deseja-se atingir, qualquer que seja o estado inicial.
} 
- todos os custos imediatos das transições partindo de um estado que não seja meta são positivos, e

- o custo inicial de todos os estados não superestimam seu custo real.

Uma forma de garantir a satisfação dessas condições é assegurar que cada estado seja sempre visitado. Isso é feito utilizando o procedimento chamado de Trial-based RTDP, que roda repetidas tentativas em que existe uma probabilidade não-nula de começar em cada estado.

Algumas variações de RTDP foram sugeridos na literatura. Um esquema que utilizou marcadores foi introduzido no algoritmo por Bonet e Geffner (2002) e Bonet e Geffner (2003), que melhorou a taxa de convergência do algoritmo. Focused RTDP (SMITH; SIMMONS, 2006) e Bounded RTDP (MCMAHAN; LIKHACHEV; GORDON, 2005) exploraram o uso dos dois limites (inferior e superior) da estimativa da função valor ótima para acelerar a taxa de convergência e "anytime performance" (habilidade do algoritmo apresentar uma política parcial que tenha um desempenho razoável em qualquer instante após o início da computação). Numa abordagem diferente, Buffet e Aberdeen (2005) e Trevizan, Cozman e de Barros $(2007,2008)$ investigaram a aplicação de RTDP para as variações de MDP com probabilidades imprecisas (que serão vistos adiante). Buffet e Aberdeen (2005) modificaram o algoritmo de RTDP para os modelos chamados BMDP, e Trevizan, Cozman e de Barros (2007, 2008) para MDPST.

\subsection{MDPIP}

O sucesso do modelo MDP não significa, no entanto, que não haja limitações. Uma das maiores limitações de MDP, que na verdade acompanha qualquer modelo probabilístico (WALLEY, 1996), é a necessidade de especificar uma grande quantidade de valores de probabilidades. Na prática, tais valores podem ser difíceis de se obter, e essa condição pode não ser atendida (UTKIN; AUGUSTIN, 2005). Uma alternativa é substituir o modelo clássico de probabilidades por probabilidades imprecisas, que permite o uso de conjuntos 
ou intervalos de probabilidades na modelagem de incertezas.

O emprego de probabilidades imprecisas em MDPs mostra-se interessante por diversos motivos. Probabilidades imprecisas facilitam a especificação do grande número de atribuições necessárias para se determinar um modelo completo (WALLEY, 1996); fornecem uma forma de modelar opiniões conflitantes de um grupo de especialistas (WALLEY, 1996); permitem abstrair detalhes menos importantes do modelo para reduzir a complexidade computacional (HARMANEC, 2002); e quando necessário, fornecem uma forma simples de realizar análise de sensibilidade em um modelo base (SHIROTA FILHO et al., 2007). Em outras ocasiões, permite lidar com dados ambíguos ou conflitantes, situações em que a forma clássica não apresenta solução satisfatória.

O primeiro a investigar imprecisão nas transições em MDP foi a tese de Silver (1963). Silver desenvolveu o processo de decisão markoviano com probabilidades ou recompensas incertas (Markov Decision Process with Uncertain Transition Probabilities or Rewards), e propôs uma formulação baseada em atualizações Bayesianas das probabilidades com a observação de novas transições. A consecutiva observação das transições permite a convergência dos valores de probabilidade para o valor real, o que resulta na construção de políticas melhores. Ele desenvolveu uma matriz de transição baseada numa distribuição multinomial beta (atualmente conhecida como distribuição de Dirichlet) para simplificar o tratamento matemático dessas atualizações. Pelo emprego do teorema de Bayes, é conhecida como abordagem Bayesiana.

O alto custo computacional das atualizações Bayesianas fez com que essa abordagem não fosse muito popular. Mais sucesso foi obtido com a formulação de teoria dos jogos de Satia e Lave Jr. (1973). Nessa construção, admite-se que a probabilidade de transição pertença a um conjunto de probabilidades, mas o valor real é incerto. O problema dessa construção é que a equação de Bellman (Eq. (2.5)) não pode ser diretamente utilizada, uma vez que a existência de um conjunto de probabilidades inviabiliza o cálculo da esperança matemática. O uso de teoria dos jogos cria uma forma de escolher uma dessas probabilidades, que pode então ser aplicada à equação de Bellman para a avaliação de 
políticas. O conceito consiste em interpretar o processo de decisão como um jogo entre o agente e a natureza, em que o agente escolhe as ações e a natureza escolhe as probabilidades. Para que a solução desse problema seja possível, é preciso supor um comportamento para a natureza, e duas formas (ou critérios) foram consideradas. A primeira, chamada de pessimista, considera que a natureza escolhe de forma a prejudicar o agente, e as probabilidades minimizam o desempenho do agente. O segundo critério é o extremo oposto. Chamado de critério otimista, supõe que a natureza escolhe a favor do agente, e os melhores valores são selecionados. Na prática, apenas o primeiro critério é utilizado pois ele determina um limite inferior para o desempenho do agente. Essa formulação é bastante parecida com técnicas utilizadas em estatística robusta, sob o nome de $\Gamma$-minimax (BERGER, 1985).

A maioria dos trabalhos recentes se baseiam em uma dessas duas formulações. Uma exceção notável é a tentativa de Harmanec (2002) de generalizar MDPs para probabilidades imprecisas que utiliza um critério de preferência parcial para lidar com as especificações imprecisas de probabilidade. Entretanto, essa abordagem apenas identifica e exclui alternativas dominadas (ou seja, as ações cujo desempenho é inferior a outra ação para qualquer valor de probabilidade), mas não diz como escolher dentre aquelas que restarem. Outro problema com essa formulação foi apontada por Cooman e Troffaes (2005), que identificaram uma falha grave no critério, que pode não ser compatível com decisões sequenciais. Outra exceção interessante é o estudo de POMDP (Partially Observable Markov Decision Process - processos de decisão markovianos sob observabilidade parcial) de Itoh e Nakamura (2007), que adotam outro critério de preferência parcial chamado de Eadmissibilidade. E-admissibilidade também foi utilizada por Cozman e Krotov (1996), em que conjuntos de distribuições Quasi-bayesianas são usadas em problemas de planjamento.

A abordagem de MDPIPs neste trabalho segue a formulação de teoria dos jogos de Satia e Lave Jr. (1973). A formulação matemática não difere muito daquela empregada para MDPs. Basiciamente, um MDPIP é um MDP em que o modelo probabilístico tradicional é substituído por um conjunto de probabilidades. Assim, um MDPIP consiste 
de uma tupla $\langle\mathcal{T}, \mathcal{S}, \mathcal{A}, K, R\rangle$, em que $\mathcal{T}, \mathcal{S}, \mathcal{A}$ e $C$ possuem os mesmos significados de MDP e:

- $K(r \mid s, a)$ é o conjunto de possíveis medidas de probabilidades de transição entre o estado $s$, para o estado $r$ quando a ação $a$ é aplicada. Adota-se que $K$ é um conjunto fechado e convexo. Nesse caso, também é chamado de conjunto credal (LEVI, 1980).

A aplicação dos critérios otimista e pessimista de Satia e Lave Jr. permitem escrever o problema de decisão sequencial como a busca de políticas que otimizem as seguintes expressões:

$$
\min _{a} \max _{P \in K}(\text { Custo Total Descontado Esperado), }
$$

quando se aplica o critério pessimista, ou então:

$$
\min _{a} \min _{P \in K}(\text { Custo Total Descontado Esperado), }
$$

quando o critério otimista é adotado. A equação de Bellman resultante para o caso pessimista é:

$$
V^{*}(s)=\min _{a} \max _{P \in K}\left(C(s, a)+\gamma \sum_{r} P(r \mid s, a) V^{*}(r)\right) .
$$

Diversos algoritmos foram propostos para MDPIP. Satia e Lave Jr. (1973) adaptaram o algoritmo de iteração de políticas ao empregar a equação de Bellman modificada da Eq. (2.16). Eles também investigaram a abordagem Bayesiana, para o qual propuseram um algoritmo baseado em árvores de decisão e aproximações sucessivas. White III e ElDeib (1994) formularam um algoritmo baseado em aproximações sucessivas e revisão de recompensas para MDPIPs cujas probabilidades são dadas através de restrições determinadas por um número finito de inequações lineares. Um algoritmo de iteração de políticas modificada usando revisão de recompensas também foi apresentada.

Cozman e Krotov (1996) consideraram um conjunto de probabilidades Quasi-Bayesiana e o critério de E-admissibilidade para decisões sequenciais em problemas de planejamento. O enfoque, nesse caso, era o planejamento online, então as fases de planejamento e execução eram intercaladas. Kurano, Yasuda e Nakagami (1999) investigaram a obtenção de 
políticas maximin Pareto-ótimas em problemas com recompensa média esperada. Bagnell, Ng e Schneider (2001) estudaram a conexão de MDPIP com controle ótimo estocástico e controle robusto $H_{\infty}$, que resultou no algoritmo que chamaram de iteração de valor robusta. Nilim e Ghaoui (2005) estudaram um problema parecido, mas permitiram o uso de conjunto não-convexos de probabilidades. O resultado foi um algoritmo de programação dinâmica robusta. Outra forma de iteração de políticas foi apresentada por Kalyanasundaram, Chong e Shroff (2002) para MDPs com recompensa média, tempo contínuo, critério maximin e transições especficidas por conjuntos compactos. Xu e Mannor (2007) apresentaram uma formulação que permite obter políticas Pareto-ótimas que ponderam o desempenho e a robustez, utilizando um algoritmo baseado em programação linear. Esse resultado, no entanto, se aplica apenas para problemas com recompensas incertas, e não probabilidades incertas. Iyengar (2005) deriva um programa linear para MDPs ambíguos (ou seja, cujas probabilidades são desconhecidas), mas requer que o problema respeite uma propriedade chamada de retangularidade.

Uma subclasse de MDPIPs foi proposta por Givan, Leach e Dean (2000), chamado de Bounded-parameter Markov Decision Process (BMDP). Nesse modelo, as incertezas das probabilidades e recompensas não são dadas por conjuntos, mas por intervalos. Esse modelo exibe uma estrutura que permite que seja resolvido com maior eficiência. Isso requer, no entanto, uma ordenação das transições. Uma forma adaptada de iteração de valor foi apresentada para esse modelo. Outro subconjunto de MDPIPs é o conjunto de MDPSTs, proposto por Trevizan, Cozman e de Barros (2007). Veremos esse modelo com um pouco mais detalhe na seção seguinte devido a sua aplicação em problemas de planejamento como forma de unificar modelos probabilísticos e não determinísticos.

Uma formulação menos relacionada é o AMDP, Algebraic Markov Decision Process. Essa é uma proposta de Perny, Spanjaard e Weng (2005) na tentativa de generalizar MDPs e incluir vários modelos quantitativos e qualitativos dentro de um mesmo formalismo. Para tal, eles abandonam certas premissas do modelo tradicional e se valem de uma estrutura baseada em semi-anéis para representação de recompensas e incertezas. Isso resulta no uso 
de conjuntos para especificação de recompensas e medidas de plausibilidade para modela incertezas. Perny, Spanjaard e Weng notam que essas são diferentes de plausibilidade e funções crença da teoria de Dempster-Shafer, sendo na verdade relacionadas ao conceito apresentado por Friedman e Halpern (1995).

\subsection{MDPST}

Um MDPST, Markov Decision Process with Set-valued Transitions, é um MDP em que as transições probabilísticas levam a um conjunto de estados em vez de um único estado. Nesse caso, a probabilidade pode ser vista como uma massa de probabilidade associada ao conjunto de estados, que no entanto não é determinado para cada estado em particular. Um MDPST é, então, especificado através de uma tupla $\langle\mathcal{T}, \mathcal{S}, \mathcal{A}, F, m, C\rangle$, em que $\mathcal{T}, \mathcal{S}$, $\mathcal{A}$ e $C$ são os mesmos para MDP e MDPIP e (TREVIZAN; COZMAn; DE BARRos, 2007):

- $F(s, a) \subseteq 2^{\mathcal{S}} \backslash \emptyset$ é uma função de transição de estados mapeando pares de estado $s$ e ação $a \in A(s)$ em conjuntos de estados alcançáveis em $\mathcal{S}$,

- $m(k \mid s, a)$ são atribuições de massa para todo $s \in \mathcal{S}, a \in A(s)$ e $k \in F(s, a)$.

Esse modelo foi proposto por Trevizan, Cozman e de Barros (2007) em conexão com problemas de planejamento probabilístico e não-determinístico. Note que o modelo se reduz a um MDP quando o conjunto $F(s, a)$ é um conjunto unitário para todo $s \in \mathcal{S}$ e $a \in A(s)$, e torna-se puramente não-determinístico quando $m(k \mid s, a)=1$ para todo $s \in \mathcal{S}$, $a \in A(s)$ e alguma transição $F(s, a)$. Dessa forma, estabelece-se um formalismo comum para ambos os modelos, que até então não tinha sido explorado.

Por ser uma forma especial de MDPIP, qualquer método desenvolvido para MDPIP pode ser usado para resolver um MDPST. Por isso, basta utilizar uma forma modificada da equação de Bellman (TREVIZAN; COZMAN; DE BARROS, 2007). Utilizando uma abordagem 
pessimista, essa equação pode ser escrita como:

$$
V^{*}(s)=\min _{a \in A(s)}\left(C(s, a)+\gamma \sum_{k \in F(s, a)} m(k \mid s, a) \min _{s^{\prime} \in k} V^{*}\left(s^{\prime}\right)\right) .
$$

O resultado dessa expressão é que toda a massa de probabilidade $m(k \mid s, a)$ é atribuída ao estado $s^{\prime}$ que possui a menor utilidade. Esse procedimento claramente obtém um resultado conservador, que pode ser utilizado como uma solução robusta para o problema. No entanto, assim como em MDPIP, em muitas situações essa abordagem é considerada demasiadamente pessimista, e criticada por abrir mão de melhores desempenhos.

Em termos de algoritmos específicos para MDPST, Trevizan, Cozman e de Barros (2008) apresentaram um algoritmo baseado em (L)RTDP, que na verdade resolve uma forma especial do modelo chamado de SSP (esse problema será explicado na seção a seguir).

\subsection{SSP}

Problemas de menor caminho estocástico (SSP, do inglês Stochastic Shortest Path), consistem na generalização do problema clássico de menor caminho (BERTSEKAS; TSITSIKLIS, 1991), que geralmente recorrem ao uso de MDP como modelo subjacente. Nesse caso, o estado meta é absorvente, ou seja, $P\left(s_{G} \mid s_{G}, a\right)=1$ e o custo $C\left(s_{G}, a\right)=0$ para todo $a \in A\left(s_{G}\right)$ (BONET; GEFFNER, 2002). A existência de uma solução é garantida sob duas condições (BERTSEKAS; TSITSIKLIS, 1991; BONET; GEFFNER, 2002):

1. existe uma política que, partindo de qualquer estado, atinge o estado meta com probabilidade 1 , e

2. todos os custos são positivos (ou possivelmente, negativos).

A importância de SSPs para o presente trabalho está no fato de que o problema de planejamento probabilístico geralmente assume essa forma. Como os estados inicial e objetivo são fixos, a solução ótima pode ser dada na forma de uma política ótima parcial, 
que escolhe as melhores ações $a^{*}(s)$ para todos os estados relevantes (ou seja, alcançáveis) segundo a política $\pi^{*}$ (BONET; GEFFNER, 2002). Um algoritmo bastante eficiente para obter soluções dessa forma é o RTDP, que será visto no próximo capítulo. 


\section{Fundamentos}

Esta seção é fruto do estágio realizado junto ao Grupo de Estatística da Universidade de Durham (Inglaterra), sob orientação do Dr. Matthias C. M. Troffaes e colaboração de Nathan Huntley. A idéia inicial era investigar os fundamentos de tomada de decisão sequencial, mais especificamente o estudo de processos de decisão markovianos, em casos em que as incertezas são modeladas através de probabilidades imprecisas.

A primeira referência a esse modelo na literatura data da década de 1960, com o trabalho de Silver (1963). Já naquela época, havia uma preocupação com a dificuldade em se determinar valores precisos para as inúmeras distribuições de probabilidades necessárias para a especificação de um processo de decisão markoviano. Nesse sentido, Silver propôs que tais incertezas fossem especificadas através de um conjunto de probabilidades, e que, através da aplicação de atualizações bayesianas com dados obtidos pela observação das transições, eventualmente convergissem para o valor preciso real.

A abordagem bayesiana mostrou-se, na época, computacionalmente inviável e tornouse relevante apenas décadas depois com o florescimento das técnicas de aprendizado por reforço. Nesse meio tempo, o uso de probabilidades imprecisas dentro do modelo markoviano de decisão tornou-se popular através da abordagem de teoria dos jogos apresentada por Satia e Lave Jr. (1973). A interpretação do problema nessa abordagem baseia-se em um jogo entre a natureza e o agente, em que o agente escolhe as ações e a natureza, os valores de probabilidade. Nessa formulação, torna-se importante a escolha para o comportamento da natureza (também chamado de critério de otimalidade), sem a qual o problema de decisão sequencial não pode ser resolvido. Em geral, adota-se uma das 
seguintes opções: o cenário otimista, também chamado de max-max, em que a natureza age de forma a colaborar com o agente (ou seja, escolhe as distribuições de probabilidade que mais favorecem o desempenho do agente), ou o cenário pessimista, ou max-min, na qual ela age de forma a competir com o agente (prejudicando o desempenho deste).

Apesar de Satia e Lave Jr. (1973) ter introduzido a abordagem de teoria dos jogos para processos de decisão markovianos com probabilidades imprecisas, os resultados mais importantes de otimalidade de convergência do algoritmo proposto são apresentado em Satia (1968). Esses resultados baseiam-se na análise do programa dinâmico derivado do problema de decisão sequencial, na qual as devidas transformações são aplicadas para satisfazer o critério escolhido para o comportamento da natureza.

É nesse ponto que inicia-se a discussão proposta no estágio realizado na Universidade de Durham. A análise de Satia (1968) concentra-se no programa dinâmico modificado pela aplicação do critério de otimalidade adotado. O problema é que a teoria desenvolvida por Bellman (2003) baseia-se em normas fundamentadas na teoria clássica (precisa) de probabilidades, que sem uma investigação mais profunda, não podem ser naturalmente estendidas para o caso com probabilidades imprecisas. A desconfiança sobre os resultados estabelecidos são reforçados por trabalhos recentes, nas quais algumas suposições adotadas por Satia (1968) mostram-se adequadas em problemas de decisão sequencial com probabilidades imprecisas. Apenas como exemplo, Seidenfeld (2004) mostra que o critério max-min (também chamado de $\Gamma$-maximin) pode levar a soluções sub-ótimas.

Apesar de ser um assunto bastante interessante, não seria viável efetuar uma discussão profunda sobre os fundamentos de decisões sequenciais neste trabalho. Dessa forma, não existe a pretensão de apresentar um texto completo e fechado sobre o assunto, até pela falta de tempo hábil para tal pesquisa. Ao invés disso, a seguir apresentam-se as principais constatações levantadas durante o trabalho efetuado, e, em alguns casos, possíveis caminhos que futuramente mereçam ser investigados. Um artigo resultante desta pesquisa, apresentado no 13th International Conference on Information Processing and Management of Uncertainty in Knowledge-Based Systems (IPMU'2010) encontra-se nos 
anexos.

\section{O critério $\Gamma$-maximin viola a equação de Bellman $\Gamma$-maximin considera que}

opções sejam classificadas pelo seu menor valor esperado, sendo posteriormente, escolhido o maior desses valores (SEIDENFELD, 2004). Esse é o critério pessimista de Satia e Lave Jr. (1973), que é adotado na maioria dos trabalhos em MDPIP e bastante popular em estatística robusta. No entanto, $\Gamma$-maximin deve ser utilizado com cautela em decisões sequenciais, pois viola o axioma de independência de von Neumann e Morgenstern ou o postulado (ou princípio) da certeza de Savage (SEIDENFELD, 2004).

Violar os axiomas que regem a teoria de escolha racional é, obviamente, uma falha grave. Isso significa que os resultados estabelecidos e geralmente aceitos de decisão racional não podem ser diretamente aplicados para o critério $\Gamma$-maximin em decisões sequenciais. Seidenfeld (2004) mostra que é possível criar situações em que um agente é induzido a realizar escolhas sub-ótimas sob esse critério. Em MDPIP, isso equivale a violar a Equação de Bellman.

Uma série de questões tornam-se aparentes com essa discussão. Primeiro, o cenário criado por Seidenfeld (2004) é relevante no contexto de MDPs? Na hipótese de se reconhecer que $\Gamma$-maximin seja uma alternativa inviável, existem critérios que possam substituí-lo à altura? Será que existem condições sob as quais $\Gamma$-maximin possa ser empregado sem violar os respectivos axiomas?

De uma forma ou outra, Г-maximin é um critério de escolha razoável em muitas situações. Casos de aversão total a risco é uma delas. Isso surge, por exemplo, em decisões médicas envolvendo a possibilidade de morte. Nesse sentido, compreender as limitações a encontrar novas maneiras de adequar o critério aos axiomas (ou talvez até modificar os axiomas de forma a comportar o critério) torna-se preferível a excluir o critério por completo. 
Alternativas ao $\Gamma$-maximin não são práticas Na literatura, existem três critérios alternativos a $\Gamma$-maximin: dominância por intervalos, maximalidade e E-admissibilidade (KIKUTI; COZMAN; CAMPOS, 2005; TROFFAES, 2007). Esses critérios são diferentes de $\Gamma$-maximin por determinarem ordenações parciais das alternativas. Ordenações parciais permitem a incomparabilidade de alternativas, que resulta em indecisão.

Para ilustrar essa situação, considere o exemplo de Seidenfeld (2004). Nesse exemplo, um agente é apresentado a três opções, $A, B$ e $C$. Os ganhos devolvidos por essas opções são dependentes da realização de um evento $e$, cuja probabilidade é desconhecida. Assim, a probabilidade $P(e)$ pode ser qualquer valor entre 0 e $1 . A$ vale 1 unidade se $e$ é verdadeiro e 0 se for falso. $B$ é a opção contrária, vale 1 se $e$ for falso e 0 se for verdadeiro. A opção $C$ vale 0,4 , independentemente da realização de $e$.

Nessa configuração, os valores esperados para $A$ e $B$ variam entre 0 e 1 (note que Seidenfeld (2004) se limita ao intervalo $0,25 \leq P(e) \leq 0,75$, mas que para o exemplo isso não é importante), enquanto que $C$ possui um ganho preciso igual a 0,4 . $\Gamma$-maximin, ao selecionar a alternativa com maior valor para o pior valor esperado, claramente opta pela solução $C$. Isso porque no pior caso, tanto $A$ quanto $B$ possuem o valor 0 , enquanto que $C$ tem um valor constante igual a 0,4 .

E-admissibilidade restringe o conjunto de opções admissíveis ao agente àquelas que são ótimas para pelo menos um valor de probabilidade. No exemplo, apenas $A$ ou $B$ exibem essa característica. O problema, nesse caso, é que E-admissibilidade mostra-se incapaz de optar entre essas duas alternativas, e um agente E-admissível fica indeciso.

Indecisão é perversa em tomada de decisão por estagnar o processo. Mesmo que ao final de um estágio um agente seja obrigado a fazer uma escolha, o critério não prescreve uma forma de realizá-lo. Isso leva a comportamentos incoerentes, ilustrado no exemplo do asno de Buridan, apresentado em Sen (1985): o asno morreu de fome ao ficar indeciso entre duas pilhas de feno.

A utilização de critérios de ordenações parciais (ou seja, qualquer alternativa atual- 
mente disponível para $\Gamma$-maximin) depende, portanto, de uma solução para indecisão. No momento, a forma que parece ser mais coerente é a linha de Cozman e Krotov (1996), em que o agente observa novas transições sempre que indecisão é atingida. Isso, no entanto, depende da possibilidade do agente postergar sua decisão, e requer que transições passadas sejam armazenadas e as probabilidades atualizadas.

\section{A forma de se escolher critérios e as implicações dessa escolha não são muito}

claras A discussão anterior leva ao claro favorecimento do critério $\Gamma$-maximin para efeitos práticos, mas em termos de comportamento do agente, em que situações deve-se considerar as demais alternativas? Na literatura, existe uma forte conexão entre políticas $\Gamma$-maximin e soluções robustas. Assim, entende-se que $\Gamma$-maximin deva ser utilizado em casos de aversão a risco, mesmo que isso implique em desempenhos inferiores. Para os demais, é difícil estabelecer os casos em que a aplicação do critério torna-se interessante. Troffaes (2007) sugere uma escolha baseada nas propriedades desejadas da solução ótima e na complexidade computacional do problema enfrentado, mas não são discutidas as implicações no desempenho obtido.

\section{Não existem algoritmos que implementem esses outros critérios Harmanec} (2002) apresenta uma solução para MDPIP baseado no critério de dominação por intervalos. Nesse critério, são comparados os intervalos de utilidade determinados pelo conjunto de probabilidades das diferentes ações, e caso uma ação seja dominada, ou seja, seu valor superior seja inferior à alguma outra opção, ela é considerada inadmissível. No entanto, Cooman e Troffaes (2005) identificaram um problema com esse critério, e foi mostrado que ele é inadequado para problemas de decisão sequencial.

Em Kikuti, Cozman e Shirota Filho (2011), são apresentados alguns algoritmos para tomada de decisão sequencial com preferências parciais. Esses algoritmos não foram propostos em conexão com a teoria de MDP, mas já apresentam um primeiro passo para a viabilização do emprego de critérios de ordenação parcial em MDPIP. Alguns resul- 
tados desse artigos (mais especificamente, a Seção 4.3) foram obtidos em conjunto com desenvolvimentos desta tese.

$\Gamma$-maximin é realmente robusto? Uma das principais defesas ao $\Gamma$-maximin é a obtenção de soluções robustas. Essa noção é tão presente na literatura, que dificilmente ela é questionada.

Um estudo que foi realizado durante esta pesquisa em conjunto com Dr. Matthias C. M. Troffaes e Nathan Huntley durante uma visita à Universidade de Durham, na Inglaterra, sugere que, pelo menos em certas situações, essa noção pode ser equivocada. Em um exemplo prático simples consistindo no arremesso sequencial de uma moeda, o desempenho de um agente $\Gamma$-maximin mostrou-se menos robusto do que um agente sob maximalidade ou E-admissibilidade. Igualmente surpreendente foi notar que o desempenho de $\Gamma$-maximin foi, em média, superior ao agente maximal ou E-admissível, contrariando toda a intuição que existe sobre o desempenho dos diferentes critérios. A parte teórica dessa pesquisa pode ser consultada no artigo Troffaes, Huntley e Shirota Filho (2010). O documento resultante desse trabalho, que ainda não foi publicado em sua forma integral, encontra-se no Apêndice II. Nota-se que o conteúdo do artigo se estende ao exemplo explicado abaixo, e diversas discussões técnicas são apresentadas. Recomenda-se a leitura do texto em anexo para maiores esclarecimentos.

Um agente aposta sequencialmente sobre o arremesso de uma moeda. A correta previsão do resultado rende o ganho de uma unidade de utilidade ao agente, enquanto que a previsão incorreta o priva de uma unidade. O agente deseja obter um desempenho ótimo, ou seja, maximizar os lucros esperados sobre toda a sequência de arremessos. Infelizmente, a moeda não pode ser considerada justa, e o viés é desconhecido.

Foram considerados 5 agentes: Bayesiano clássico, $\Gamma$-maximin, maximal, E-admissível, e dominância por intervalos. Para tornar o problema mais interessante, foi permitido que os agentes aprendessem com as transições (ou seja, com os arremessos da moeda). O agente Bayesiano, restrito ao uso de probabilidades precisas, inicia o processo com uma 


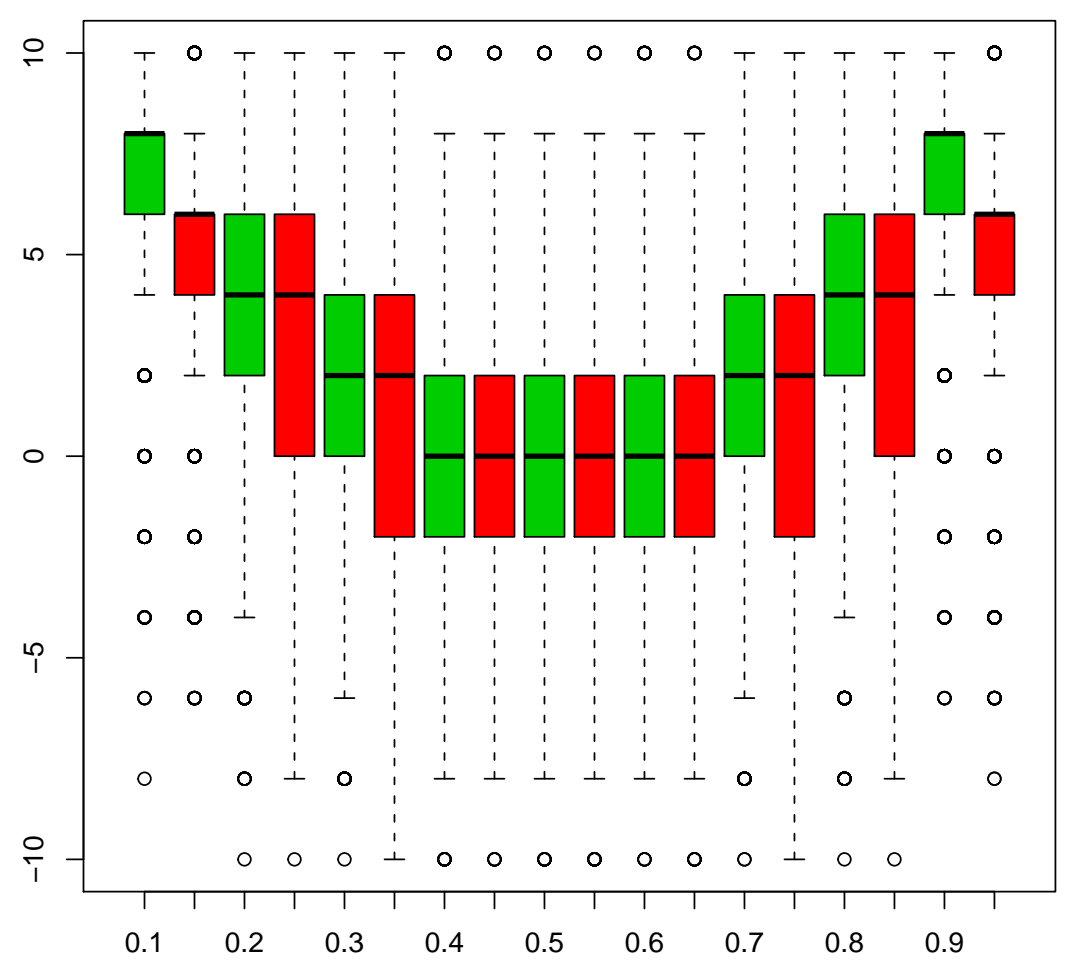

Figura 3.1: Box-plot do desempenho médio do agente em 10.000 experimentos de 10 arremessos de moeda.

distribuição uniforme. Os demais agentes consideram o modelo impreciso de Dirichlet (WALLEY, 1996; BERNARD, 2005), e empregam uma distribuição priori vacuosa (vacuous prior, todos os valores de probabilidade são permitidos). Nesse caso simples, o agente Bayesiano clássico e $\Gamma$-maximin prescrevem as mesmas ações (chamaremos de agente sob ordenação completa), fato que também ocorre entre o maximal, E-admissível e dominância por intervalos (que indicaremos como agente sob ordenação parcial). 10,000 sequências de 10 arremessos foram simulados para diferentes valores de probabilidade de viés da moeda, e o resultado do ganho acumulado é apresentado em um box-plot na Figura 3.1. O eixo horizontal representa os diferentes viés da moeda. Foram consideradas 9 discretizações, de 0,1 a 1 . O eixo vertical representa o ganho acumulado durante a sequência de 10 arremessos. Os dados em verde representam o agente sob ordenação completa e em vermelho, o agente sob ordenação parcial. Sendo um box-plot, a linha central grossa é a 
mediana do conjunto de dados, e a caixa compreende a região delimitada pelos quartis superior e inferior. As hastes se estendem até as cercas superior e inferior, a partir da qual os dados são considerados valores atípicos. Esses são representados pelos círculos vazios.

Na simulação da Figura 3.1, o agente sob ordenação parcial foi obrigado a fazer uma escolha mesmo sob indecisão. Como os critérios não prescrevem formas de desempate, foi utilizado um sorteio aleatório sobre as opções adimissíveis. Para esse caso simples essa abordagem é suficiente, mas um problema mais complexo provavelmente exigiria uma melhor investigação.

Analisando-se o resultado, nota-se que os desempenhos foram bastante semelhantes, o que era esperado pela simplicidade do problema considerado. Um fato interessante é que o agente sob ordenação completa (verde) obteve um desempenho consideravelmente superior para valores extremos de viés. Essa diferença não era esperada, e acredita-se que seja devido à convergência mais rápida do modelo Bayesiano clássico.

Obtendo-se esse resultado um pouco decepcionante para probabilidades imprecisas, decidiu-se investigar uma outra situação, um pouco mais favorável ao modelo impreciso. Uma ação foi adicionada, e quando o agente sob ordenação parcial encontrasse uma situação de indecisão, foi permitido que ele se abstesse da decisão e observasse uma transição. O resultado dessa simulação é apresentado na Figura 3.2

Novamente, os dados em verde correspondem ao agente sob ordenação completa, e em vermelho, ao agente sob ordenação parcial. Como não houve mudança para o agente sob ordenação completa (verde), o resultado nesse caso é exatamente igual ao da Figura 3.1. O desempenho do agente sob ordenação parcial (vermelho), no entanto, modificou-se profundamente. Primeiro, o desempenho nos casos de viés extremos foram reduzidos levemente. Segundo, nota-se que a dispersão diminuiu bastante. Essa redução da dispersão é tão significativa que o agente sob ordenação parcial na verdade tornou-se mais robusto que o agente sob ordenação completa. Como nesse exemplo um agente $\Gamma$-maximin age da mesma forma que um agente sob ordenação completa, o agente $\Gamma$-maximin não exibe a 


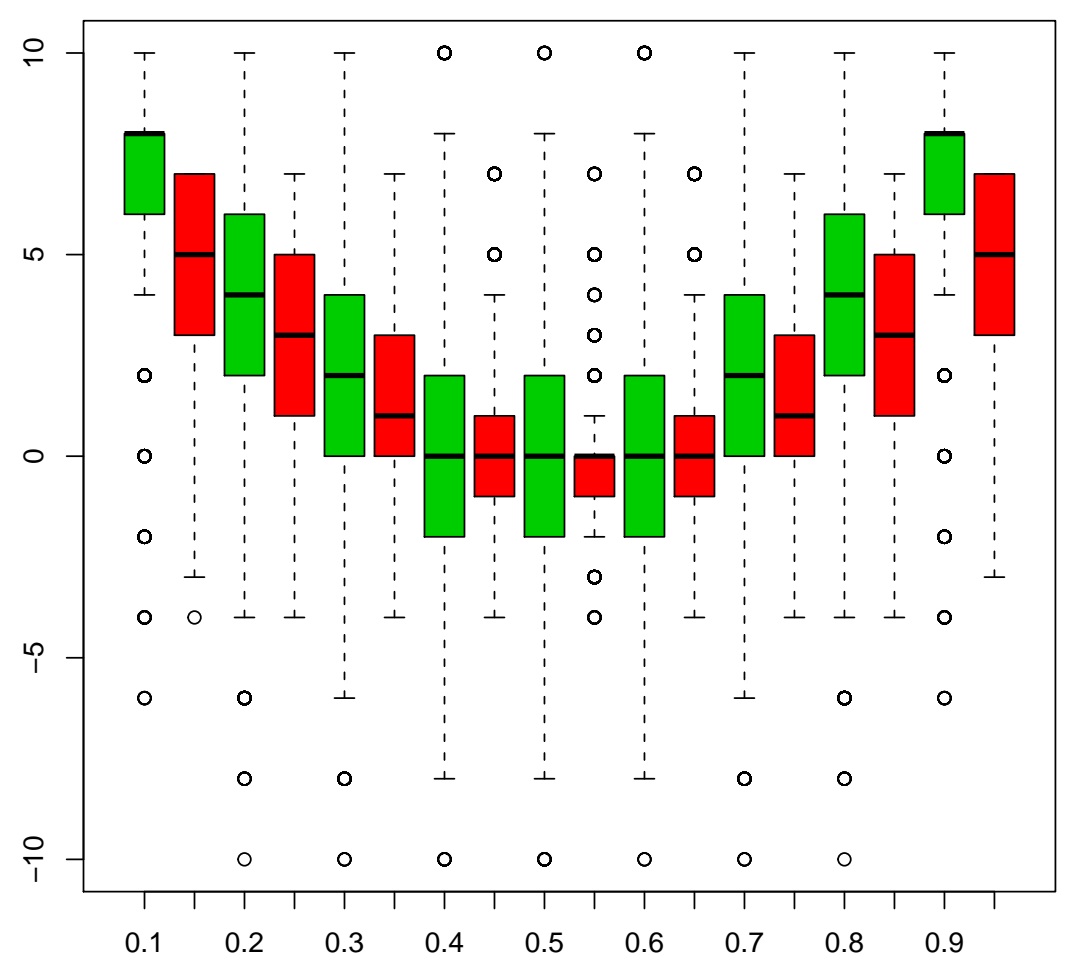

Figura 3.2: Box-plot do desempenho médio do agente permitindo a observação de transições em situações de indecisão.

robustez tão característica desse critério. 


\section{Planejamento}

Planejamento é a área da inteligência artificial interessada na elaboração de planos, ou seja, na organização de passos visando o alcance de um objetivo. Originalmente, o problema de planejamento consistia na busca de uma sequência de operadores lógicos ou ações que transformassem um estado inicial em um estado objetivo desejado (LAVALLE, 2006). Essa construção pressupunha, além de estados inicial e objetivo(s) bem definidos, a inexistência de qualquer incerteza associada ao problema investigado. Suposições como esta última eventualmente mostraram-se demasiadamente restritivas, e novas abordagens permitindo a investigação de problemas mais complexos passaram a ser desenvolvidas. Como resultado, houve uma segmentação e especialização do problema de planejamento, surgindo, assim, classes como planejamento não-determinístico e planejamento probabilístico.

Conforme apontado por LaValle (2006, Seção 1.1), o termo planejamento possui vários significados dependendo da abordagem utilizada - a comunidade de robótica entende de uma forma, a de controle de outra, que difere levemente do entendimento da IA. Este trabalho adota a noção geralmente aceita em inteligência artificial, e assim, considera os seguintes elementos básicos na descrição de um problema de planejamento (LAVALLE, 2006):

Estados: Representam todas as possíveis situações, ou seja, as configurações do ambiente.

Tempo: Indica estágios de decisão. Muitas vezes, uma representação explícita do tempo não é necessária (o instante particular não é importante), bastando uma referência para a correta sequência das ações. 
Ações: Manipulam os estados, afetas as probabilidades, e são prescritas através de planos.

Estado inicial e estado meta: São os elementos do conjunto de estados que identificam, respectivamente, as configurações inicial e final do processo. Em alguns casos, podem ser dados na forma de conjuntos.

Critério: Codifica o resultado desejado do plano em termos dos estados e das ações que são executadas. Duas linhas são geralmente seguidas: a) viabilidade, quando se busca um plano que causa a obtenção de um estado meta independentemente da sua eficiência; b) otimalidade, quando o interesse é encontrar um plano viável mas que também otimiza o desempenho segundo uma norma de avaliação definida.

Plano: Especifica a estratégia ou o comportamento do agente ou tomador de decisão. No caso mais simples, um plano limita-se a uma sequência de ações. Porém, quando não é possível prever estados futuros, ele se apresenta na forma de uma função (ou mapa) que atribui ações para estados, que é chamada de política.

Alguns autores (e.g. LaValle (2006)) ainda definem as noções de algoritmos, planejadores e as formas de emprego de planos, mas postergamos essas explicações para seções futuras. A seguir, são apresentadas características do problema que são importantes para a identificação da classe de planejamento à qual ele pertence.

\subsection{Caracterização de Domínios}

A larga gama de aplicações da IA e de planejamento leva a um rol bastante extenso de domínios de interesse. Consequentemente, as mais diversas exigências são encontradas, que no entanto, podem ser agrupadas em classes e tratadas de forma semelhante. Russell e Norvig (2010) listam algumas características que permitem determinar as classes de domínios sob as quais as mesmas técnicas podem ser utilizadas. A seguir, revisam-se brevemente essas características para identificar o problema investigado neste trabalho. 
Observabilidade A observabilidade indica a forma com que o ambiente é percebido pelo agente. Pode ser completamente observável, quando o agente tem acesso a todas as informações necessárias para determinar em qual estado ele se encontra; parcialmente observável, quando apenas parte dessas informações estão disponíveis; ou não-observável, quando elas não estão presentes ou deseja-se obter planos que não dependam da sua disponibilidade.

A questão da observabilidade é de fundamental importância dentro de problemas de planejamento e IA, principalmente quando são considerados agentes físicos atuando no mundo real. Ao contrário de ambientes simulados, em que informações sobre o meio estão sempre disponíveis (pois são determinados pela simulação), no mundo real essa disponibilidade depende, por exemplo, da existência e da confiabilidade de sensores ou a entrada manual de dados por um operador. Quando uma parte desses sensores são inexistentes (seja pela impossibilidade técnica de se realizar uma medição, a falta de recursos para adquirir o equipamento, ou o simples esquecimento de incluir o sensor durante a construção), o agente deixa de ter acesso às informações correspondentes, e o estado do ambiente não pode ser diretamente determinado. No entanto, mesmo com a presença dos sensores os dados podem não ser confiáveis (devido à presença de ruído ou mal-funcionamento), e o agente deve dispor de técnicas para avaliar essas informações. Problemas desse tipo são ditos parcialmente observáveis, e geralmente requerem o uso de uma função para estimar o estado em que o agente se encontra (MONAHAN, 1982; BOUTILIER; HANKS; DEAN, 1999; ROSS; CHAIB-DRAA; PINEAU, 2008; RUSSELL; NORVIG, 2010). Um caso extremo é a ausência completa de sensoreamento (não-observabilidade), que, associado a um ambiente cuja dinâmica não é bem conhecida, torna a tarefa de planejamento bastante difícil. Nesses casos, é comum que se exija que o agente consiga alcançar o objetivo a partir de qualquer estado atingível (RUSSELL; NORVIG, 2010). Problemas parcialmente observáveis são importantes em conexão com a robótica, e não serão investigadas neste trabalho. O enfoque será em problemas simulados, e portanto, em problemas completamente observáveis. 
Sequencialidade Em um cenário episódico, a experiência do agente é dividida em episódios atômicos. Tudo que acontece durante um episódio fica contido nele mesmo, e não afeta episódios futuros. Por outro lado, em um ambiente sequencial, os efeitos das ações extrapolam a duração do episódio e afetam (possivelmente todos os) estados futuros.

Problemas de planejamento são essencialmente sequenciais. Apesar de cada decisão poder ser encarada como um problema episódico, é a interação entre as escolhas passadas e as possibilidades futuras que torna o problema interessante. Além disso, problemas episódicos podem ser tratados utilizando técnicas mais simples, que fogem do escopo de planejamento.

Dinâmica Se o ambiente se modifica enquanto o agente delibera sobre a sua próxima ação, ele é considerado dinâmico. Caso contrário, ele é estático. Problemas dinâmicos são mais difíceis, pois o agente precisa constantemente verificar o estado em que ele se encontra (RUSSELL; NORVIG, 2010). Apesar de ser o caso mais simples, neste trabalho adota-se a suposição de ambientes estáticos. Essa suposição não impede, por exemplo, a modelagem de eventos independentes do agente (também chamados de eventos exógenos), que podem modificar a evolução esperada do sistema (BOUTILIER; HANKS; DEAN, 1999).

Continuidade Um parâmetro discreto é aquele que possui um conjunto (finito) bem determinado de valores. Ao contrário, um parâmetro contínuo pode ser associado a um número infinito de valores dentro de um intervalo. Esses conceitos podem ser aplicados ao estado do ambiente, à forma com que se lida com o tempo, e às ações e percepções do agente. Sem perda de expressividade pode-se adotar o caso discreto, bastando escolher uma discretização do parâmetro contínuo tão pequena quanto necessária.

Quantidade de Agentes Problemas em que existe apenas um agente atuando sobre o sistema são ditos de agentes simples. Quando existe mais de um agente, o problema é considerado multiagente. Alguns problemas multiagentes podem ser modelados usando 


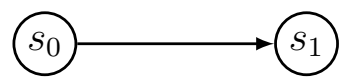

Figura 4.1: Representação gráfica de uma transição determinística

modelos de agentes simples ao incorporar a influência dos demais agentes dentro da dinâmica do sistema ou como perturbações às ações do agente principal.

Modelo de Transição Uma transição indica a mudança do sistema de um estado de existência para um outro. Dentre as características dos domínios, essa será a mais explorada neste trabalho. O modelo de transição está fortemente associado à evolução do sistema, e portanto, à influência exercida pelas escolhas do agente sobre a modificação do estado. Dessa forma, transições são dadas também em função da ação aplicada, e não somente em termos dos estados passados. Assim, por exemplo, observa-se a transição do estado $s$ para o estado $s^{\prime}$ quando o agente executa a ação $a$. Três casos são considerados:

- Quando o próximo estado $s^{\prime}$ puder ser completamente determinado pelo estado inicial $(s)$ e a ação executada $(a)$, ou seja, $s^{\prime}=T(s, a)$, em que $T$ denota uma função de transição, o domínio é chamado de determinístico. Utilizando uma representação gráfica em que círculos denotam estados e arcos denotam transições, a Figura 4.1 representa uma transição determinística.

- Ambientes estocásticos são aqueles em que distribuições de probabilidades são utilizadas para indicar as chances de ocorrência dos estados futuros, o que permite apenas uma expectativa ou esperança sobre a realização do próximo estado. Dessa forma, diferentemente do caso determinístico, é impossível antecipar o próximo estado. A função de transição, nesse caso, devolveria um valor de probabilidade, ou seja, $T\left(s, a, s^{\prime}\right) \rightarrow[0,1]$. A Figura 4.2 apresenta um exemplo de representação gráfica de uma transição probabilística.

- Uma outra forma de assinalar incertezas é através do modelo não-determinístico. Esse modelo diferencia-se do estocástico ao não especificar probabilidades aos possí- 


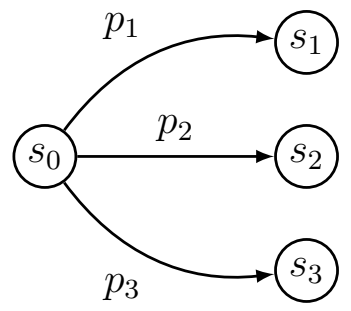

Figura 4.2: Representação gráfica de uma transição probabilística

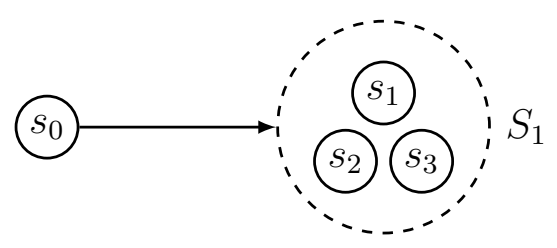

Figura 4.3: Representação gráfica de uma transição não-determinística

veis estados futuros. Ao invés disso, considera-se uma lista, e nenhuma suposição é feita sobre a possibilidade de ocorrência dos estados particulares. $T(s, a) \rightarrow 2^{\mathcal{S}}$, em que $2^{\mathcal{S}}$ é o conjunto das partes de $\mathcal{S}$, denota esse tipo de transição. Um exemplo de representação gráfica de uma transição não-determinísica é apresentado na Figura 4.3 .

Idealmente, todo problema seria suficientemente conhecido para que os efeitos das ações sobre os estados fossem precisamente determinados (ou seja, determinísticos). Infelizmente, a realidade não é tão simples. Existem casos em que o tempo e os recursos são escassos, ou o problema é demasiadamente complexo, tornando impossível o trabalho de coletar dados e detalhar o modelo a ponto de se obter o determinismo (e.g. previsão do tempo). Ou ainda, pode ser que o ambiente seja naturalmente probabilístico, e sob a influência de uma ação, ora evolua para um estado, ora evolua para outro (e.g. um sorteio, o arremesso de um dado ou de uma moeda). Nessas situações, tornou-se padrão utilizar modelos probabilísticos de transição. Mesmo sendo adequada para a maioria dos problemas, a representação de incertezas através de probabilidades não é um consenso e existem outras formas de representação. O não-determinismo é um caso, e será discutido adiante. Nas próximas seções, também será detalhado como a escolha do modelo de 
transição afeta o problema de planejamento.

O restante deste trabalho assume que os problemas são completamente observáveis, sequenciais, estáticos, discretos e de agente simples. Alguma liberdade será dada para o modelo de transição, já que será permitido determinismo, não-determinismo e estocasticidade. Para esclarecer como essa escolha influi no problema, as próximas seções introduzem as classes de planejamento que ela determina.

\subsection{Planejamento Clássico}

Problemas determinísticos (completamente observáveis, estáticos, sequenciais e de agente simples) são objeto de estudo de planejamento clássico (GEFFNER, 2002). Considerados a forma mais simples de planejamento, são caracterizados pelos seguintes elementos (BONET; GEFFNER, 2001a; GEFFNER, 2002):

C1. Um conjunto finito e discreto de estados $\mathcal{S}$

C2. Uma situação inicial dada por um estado $s_{0} \in \mathcal{S}$

C3. Uma meta dada na forma de um conjunto não-vazio $S_{G} \subseteq \mathcal{S}$

C4. Um conjunto de ações $A(s) \subseteq \mathcal{A}$, aplicáveis em cada estado $s \in \mathcal{S}$

C5. Uma função de transição de estados determinística $T: \mathcal{S} \times \mathcal{A} \rightarrow \mathcal{S}$

C6. Uma função custo positiva $C(a, s)$, o custo de executar a ação $a$ no estado $s$

A observabilidade completa e o determinismo das transições implicam em um problema sem incerteza. Dessa forma, dado um estado inicial e uma ação nele aplicado, o próximo estado torna-se completamente determinado e dado por $s_{i}=T\left(s_{i-1}, a_{i-1}\right)$. Uma solução consiste em uma sequência de ações, que deve ser aplicada a partir do estado inicial. Soluções são ótimas quando o custo acumulado $\sum_{i=0}^{n} C\left(s_{i}, a_{i}\right)$ sobre todo o processo de comprimento $n$ for mínimo, que no modelo de planejamento clássico (em que os custos são iguais para todas as transições) é equivalente à solução de caminho mínimo (GEFFNER, 
2002). Alguns autores (e.g. Russell e Norvig (2010)), se referem a planejamento clássico apenas quando a representação de estados e ações for fatorada (a definição de uma representação fatorada será apresentada na Seção 4.4), mas não adota-se essa convenção neste trabalho.

A previsibilidade do processo determinístico faz com que a obtenção de soluções seja possível através de uma simples busca pelo espaço de estados (GEFFnER, 2002). Tradicionalmente, os métodos de solução têm sido baseados em algoritmos de divisão e conquista (BONET; GEFFNER, 2001a), mas formas alternativas incluem resolução através de satisfazibilidade booleana, satisfação de restrições e cálculo de situações (BONET; GEFFNER, 2001a; GEFFNER, 2002; RUSSELL; NORVIG, 2010). As formas clássicas consideram buscas progressivas a partir do estado inicial, buscas regressivas a partir dos estados meta, ou buscas bidirecionais, sendo que estas últimas incorrem o custo de encontrar um ponto de conexão entre as duas árvores de busca (LAVALLE, 2006). Para expandir a árvore, pode-se usar métodos tradicionais como busca em nível ou busca em profundidade, ou ainda busca gulosa, busca heurística ou o algoritmo de Dijkstra (LAVALLE, 2006).

\subsection{Planejamento sob Incerteza}

O mundo real está repleto de incertezas, e em muitos problemas, esse fato não pode ignorado. Na verdade, a maioria dos problemas reais requerem o reconhecimento dessas incertezas, e métodos de planejamento clássico tornam-se inapropriados (CIMATTI; ROVERI; TRAVERSO, 1998; ZHAO; SUN; YIN, 2007).

Incertezas podem afetar o problema de duas formas (GEFFNER, 2002; LAVALLE, 2006; RUSSELL; NORVIG, 2010): alteram a confiabilidade dos sensores (observabilidade) e a previsibilidade do processo (modelo de transição). Como já foi dito anteriormente, este trabalho não abordará o problema da observabilidade, apesar de ser uma questão importante. Concentra-se no tratamento de incertezas do modelo de transição. A seguir, são introduzidas as duas principais classes de planejamento sob incerteza, o modelo não- 
determinístico e o modelo probabilístico.

\subsubsection{Planejamento Não-determinístico}

O termo 'não-determinismo' (às vezes 'não-determinismo puro') usado em planejamento é, de certa forma, uma denominação infeliz (TREVIZAN; COZMAN; DE BARROS, 2007). Nãodeterminismo usualmente se contrapõe ao determinismo, e na literatura essa concepção está geralmente associado à teoria de probabilidades ${ }^{1}$. Porém, dentro de planejamento esse termo tem uma conotação própria, e constitui uma importante classe de problemas.

No modelo de planejamento clássico, ações induzem efeitos que levam o sistema de um estado inicial para um novo estado. Esse novo estado é único, e determinável através de uma função de transição. Por outro lado, no modelo não-determinístico os efeitos induzidos pelas ações podem levar o sistema a um conjunto de possíveis estados, sem que haja nenhuma ordenação sobre suas ocorrências (BONET; GEFFNER, 2001b). A definição de um problema de planejamento não-determinístico é dado em termos de (TREVIZAN; COZMAN; DE BARROS, 2007):

C1. Um conjunto finito e discreto de estados $\mathcal{S}$

C2a. Um conjunto não-vazio de possíveis situações iniciais $\mathcal{S}_{0} \subset \mathcal{S}$

C3. Uma meta dada na forma de um conjunto não-vazio $S_{G} \subseteq \mathcal{S}$

C4. Um conjunto de ações $A(s) \subseteq \mathcal{A}$, aplicáveis em cada estado $s \in \mathcal{S}$

C6. Uma função custo positiva $C(a, s)$, o custo de executar a ação $a$ no estado $s$

NDET. uma função de transição de estados valorada em conjuntos $F: \mathcal{S} \times \mathcal{A} \rightarrow 2^{\mathcal{S}}$

A área de planejamento não-determinístico é separada em diferentes sub-áreas conforme a abordagem adotada ao problema descrito acima. Planejamento contingente resolve esse

\footnotetext{
${ }^{1}$ Nesse sentido, é válida a sugestão de Trevizan, Cozman e de Barros (2007) que defende o termo 'incerteza Knightiana pura' como uma alternativa mais apropriada.
} 
problema através de um plano condicional gerado a partir da incerteza sobre o estado inicial e os efeitos das ações, dada a possibilidade de observar algumas variáveis do problema (ZHAO; SUN; YIN, 2007; RUSSELL; NORVIG, 2010). Planejamento conformante estende esse problema para o caso sem nenhum sensoreamento (GEFFNER, 2002; RUSSELL; NORVIG, 2010), e planos devem levar a um estado meta independentemente do estado inicial ou das transições realizadas (CIMATTI; ROVERI; TRAVERSO, 1998; GEFFNER, 2002; ZHAO; SUN; YIN, 2007). Planejamento contínuo e replanejamento podem ser usados quando o ambiente é desconhecido (RUSSELL; NORVIG, 2010).

\subsubsection{Planejamento Probabilístico}

Uma outra forma de lidar com incertezas é através de modelos probabilísticos. Planejamento probabilístico adota essa representação como forma de especificar as incertezas associadas às transições entre estados. Formalmente, utiliza-se uma atribuição ou uma distribuição de probabilidades para indicar as chances de ocorrência de certo evento. O problema é descrito através de (TREVIZAN; COZMAN; DE BARROS, 2007):

C1. Um conjunto finito e discreto de estados $\mathcal{S}$

C2a. Um conjunto não-vazio de possíveis situações iniciais $\mathcal{S}_{0} \subset \mathcal{S}$

C3. Uma meta dada na forma de um conjunto não-vazio $S_{G} \subseteq \mathcal{S}$

C4. Um conjunto de ações $A(s) \subseteq \mathcal{A}$, aplicáveis em cada estado $s \in \mathcal{S}$

C6. Uma função custo positiva $C(a, s)$, o custo de executar a ação $a$ no estado $s$

PROB1. uma distribuição de probabilidades $P_{0}$ sobre os possíveis estados iniciais $\mathcal{S}_{0}$

PROB2. uma função de transição de estados probabilística $F: \mathcal{S} \times \mathcal{A} \times \mathcal{S} \rightarrow[0,1]$

A presença de incertezas faz com que os estados futuros deixem de ser completamente previsíveis, e sequências de ações tornam-se inadequadas como soluções para o problema. Em vez disso, usam-se políticas, que são mapas de estados para ações. A forma mais 
comum de lidar com esses problemas é através de um processo de decisão Markoviano, que será analisado no próximo capítulo.

\subsection{Representação Enumerativa vs Fatorada}

Os modelos até agora considerados utilizam representação explícita, extensiva ou enumerativa do espaço de estados (BOUTILIER; HANKS; DEAN, 1999; BOUTILIER; DEARDEN; GOLDSZMIDT, 2000; DELGADO, 2010). Nesse tipo de representação, os estados são listados explicitamente através de uma enumeração, sendo que o índice $i$ da notação $s_{i}$ (ou seja, a posição na lista) é suficiente para identificar cada estado.

Um problema da representação enumerativa é sofrer a maldição da dimensionalidade (BOUTILIER; DEARDEN; GOLDSZMIDT, 2000), um conceito introduzido por Bellman (RUSSELL; NORVIG, 2010), e que é de grande importância em problemas que lidam com espaços de grandes dimensões. Segundo esse conceito, a quantidade de estados necessários para representar o sistema de interesse aumenta exponencialmente com a dimensionalidade, ou seja, com a quantidade de características necessárias para descrever o problema (BOUTILIER; DEARDEN; GOLDSZMIDT, 2000).

Imagine, por exemplo, o problema de localização unidimensional. Apenas uma variável é necessária para caracterizar esse domínio, justamente a posição do objeto ao longo da dimensão. Se o espaço for discretizado em $N$ elementos, teremos então $N$ estados. Considere agora um problema de localização bidimensional. Nesse caso, são necessários dois parâmetros para caracterizar o espaço. Se cada dimensão for dividida em $N$ partições, serão necessários $N^{2}$ estados. Em $d$ dimensões, serão $N^{d}$ estados. Para domínios com poucos parâmetros e partições pequenas, a quantidade de estados ainda é manejável. Mas se algumas poucas partições forem numerosas, podem tornar o problema inviável.

Representações enumerativas são populares em pesquisa operacional, pois é comum que o modelo seja elaborado por um especialista exclusivamente para resolver o problema tratado (BOUTILIER; HANKS; DEAN, 1999). Dessa forma, estados considerados irrelevantes 
são eliminados, e novos estados podem ser adicionados de forma a agrupar conjuntos de estados semelhantes. Assim, o espaço enumerativo mantém-se manejável (BOUTILIER; HANKS; DEAN, 1999). Planejamento, porém, tem um enfoque diferente, e frequentemente o problema é proposto na ausência de um especialista (BOUTILIER; HANKS; DEAN, 1999), fazendo com que representações implícitas ou fatoradas passem a ser de maior interesse.

Em representações fatoradas, características importantes do problema são identificadas e associadas a variáveis (também chamada de variáveis de estado (DELGADO, 2010)). Essas variáveis passam a ser utilizadas para descrever o problema. Assim, em vez de uma única variável que pode assumir muitos valores, trabalha-se com algumas variáveis que assumem poucos valores (BOUTILIER; HANKS; DEAN, 1999).

Representações Relacionais Representações fatoradas (ou estruturadas (SANNER, 2008)) utilizam características; representações relacionais estendem essa formulação para permitir que propriedades de estados e ações sejam especificadas através de relações entre objetos do domínio (SANNER, 2004). Uma das linguagens mais populares para representação relacional é o PDDL (SANNER, 2004), que é discutido na próxima seção.

\subsection{PDDL}

A Linguagem de Definição de Domínios de Planejamento, ou PDDL (Planning Domain Definition Language), surgiu da necessidade de se estabelecer uma abordagem padrão para a descrição e desenvolvimento de problemas em planejamento (MCDERMOTT, 2000). Até então, cada iniciativa admitia suas próprias premissas, tornando impossível a comparação de resultados. A constatação desse problema pela comunidade resultou na criação de uma competição de planejamento, e na elaboração de uma linguagem padrão para descrição de problemas.

Assim, surgiu o PDDL. Concebida para ser a linguagem oficial para especificação de problemas na competição de planejamento do AIPS'98 (que posteriormente foi conside- 
rada a primeira edição da Competição Internacional de Planejamento (IPC - International Planning Competition)) (MCDERMOTT et al., 1998), PDDL foi elaborada para ser uma especificação neutra, em que cada parte da representação fosse uma peça necessária da descrição das possíveis ações e seus efeitos (MCDERMOTT, 2000). Incorporando muitas idéias usadas no planejador de ordem parcial UCPOP (Universal Conditional Partial Order Planner) desenvolvido pela Universidade de Washington (BARRETT et al., 1995), a expressividade da linguagem é comparável ao ADL de Pednault para proposições e UMCP para ações (MCDERMOTT et al., 1998). A linguagem também suporta ações básicas STRIPS, efeitos condicionais, quantificação universal sobre universos dinâmicos, axiomas de domínio sobre teorias estratificadas, especificações sobre restrições de segurança, especificações de ações hierárquicas compostas por subações e submetas, e gerenciamento de múltiplos problemas em múltiplos domínios usando differentes subconjuntos da linguagem (MCDERMOTT et al., 1998). A notação sintática utiliza a forma estendida Bacchus-Naur (EBNF), que é descrita em McDermott et al. (1998).

Versões do PDDL PDDL ocorre em várias versões, sendo que praticamente uma nova versão era liberada a cada edição da competição. A versão original, utilizada na competição de 1998, foi a versão 1.2, descrita em McDermott et al. (1998). Versões subsequentes, 2.1 (FOX; LONG, 2003), 2.2 (EDELKAMP; HOFFMANN, 2004), 3.0 (GEREVINI; LONG, 2005, 2006) e 3.1 (HELMERT, 2008) constroem sobre uma versão simplificada, que foi utilizada na segunda edição, mas que não foi liberada como uma versão oficial. A versão 2.1 trouxe inovações bastante controversas, gerando uma série de críticas negativas da comunidade (vide (BACCHUS, 2003; BODDY, 2003; GEFFNER, 2003; MCDERMOTT, 2003; SMITH, 2003)). Porém, essa é a versão base utilizada neste trabalho, pois é sobre ela que a extensão probabilística é construída. A sintaxe e a semântica completa podem ser encontradas em Fox e Long (2003). 


\subsection{PPDDL}

Até a edição de 2004, a competição de planejamento contemplava apenas domínios determinísticos, e portanto, problemas de planejamento clássico. Porém, a partir dessa edição, domínios probabilísticos (e posteriormente, também outras formas de planejamento sob incerteza) passaram a fazer parte da competição, o que exigiu a adequação da linguagem. Para tal, foi criado o PPDDL, a Linguagem de Definição de Domínios de Planejamento Probabilístico, a extensão probabilística do PDDL. Em essência, PPDDL é a versão 2.1 do PDDL com a adição de efeitos probabilísticos e algumas outras construções necessárias para determinar um MDP. Descrevemos a seguir a sintaxe e a semântica pretendida de efeitos probabilísticos, que permitem a utilização de probabilidades para modelar incertezas. As demais estruturas da linguagem podem ser encontradas no manual escrito por Younes e Littman (2004).

Efeitos probabilísticos O suporte a efeitos probabilísticos é feito através da seguinte construção:

(probabilistic $p_{1} e_{1} \ldots p_{k} e_{k}$ )

A semântica pretendida é que cada efeito $e_{i}$ ocorre com probabilidade $p_{i}$ (YOUNES; LITTMAN, 2004). As restrições usuais sobre probabilidades são exigidas, isto é, $p_{i} \geq 0 \mathrm{e}$ $\sum_{i=1}^{k} p_{i}=1$.

\subsection{PDL1 e PDL2}

A quinta edição do IPC inaugurou as sessões de planejamento não-determinístico e planejamento conformante (BONET; GIVAN, 2005). A inclusão dessas classes exigiu uma revisão do PPDDL para o acréscimo de efeitos não-determinísticos, necessários para a descrição desses problemas. Esses efeitos foram adicionados por Bonet e Givan (2005) à sintaxe da 
linguagem através da seguinte construção:

$$
\text { (oneof } \left.e_{1} e_{2} \ldots e_{n}\right)
$$

em que $e_{i}$ são efeitos determinísticos em PDDL. A semântica é que, quando for executado tal efeito, um dos $e_{i}$ é aleatoriamente escolhido e aplicado ao estado atual (BONET; GIVAN, 2005).

A sintaxe proposta por Bonet e Givan (2005) era muito clara em um ponto. Os efeitos $e_{i}$ que compunham tanto os efeitos probabilísticos quanto os efeitos não-determinísticos deveriam ser determinísticos. Isso significa que a única forma sintaticamente correta de obter efeitos probabilísticos e não-determinísticos em um mesmo domínio é utilizando ações probabilítiscas e ações não-determinísticas em um mesmo problema. Contudo, essa construção não foi utilizada, e os domínios da competição sempre se mostraram puramente probabilísticos ou não-determinísticos (TREVIZAN; COZMAN; DE BARROS, 2008).

Os primeiros a notarem a possibilidade de uma extensão semântica foram Trevizan, Cozman e de Barros (2008). Ao perceberem que o encadeamento de efeitos probabilísticos e não determinísticos em PPDDL possibilitavam a obtenção de modelos mais gerais de incertezas, propuseram uma formulação que resultava em um MDPST (vide Seção 2.5). Chamando o PPDDL 1.0 com a extensões não-determinísticas de Bonet e Givan (2005) de Planning Definition Language $1.0\left(\mathrm{PDL}_{1}\right)$, ou seja, Linguagem de Definição de Planejamento 1.0, permitiram a ocorrência de efeitos não-determinísticos dentro de efeitos probabilísticos, o que resultou no $\mathrm{PDL}_{2}$. Essa linguagem, além de permitir a descrição de incertezas mais gerais que o $\mathrm{PDL}_{1}$, também permitia a unificação de problemas de planejamento probabilístico e não determinístico. A sintaxe de uma ação não-determinísticas segundo Trevizan, Cozman e de Barros (2008) é:

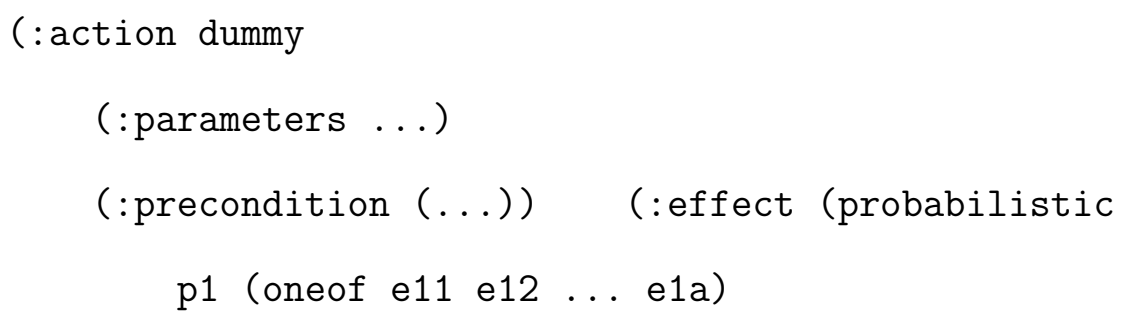




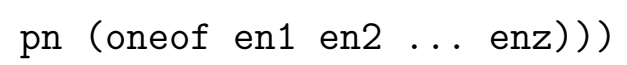

\subsection{PDL3}

Uma forma natural de estender o $\mathrm{PDL}_{2}$ é permitir que efeitos ocorram encadeados em qualquer ordem. A pergunta passa a ser qual a estrutura base necessária para codificar esse tipo de problema. Trevizan, Cozman e de Barros (2008) mostram que um MDPST não é mais adequado, e um MDPIP deve ser considerado. Veremos a seguir como isso ocorre.

A ocorrência de efeitos não-determinísticos dentro de efeitos probabilísticos foi vista na seção anterior e continua a mesma. Agora, a diferença é a possibilidade de operadores probabilísticos dentro de não-determinísticos. A construção (extraída de Trevizan, Cozman e de Barros (2008)) é:

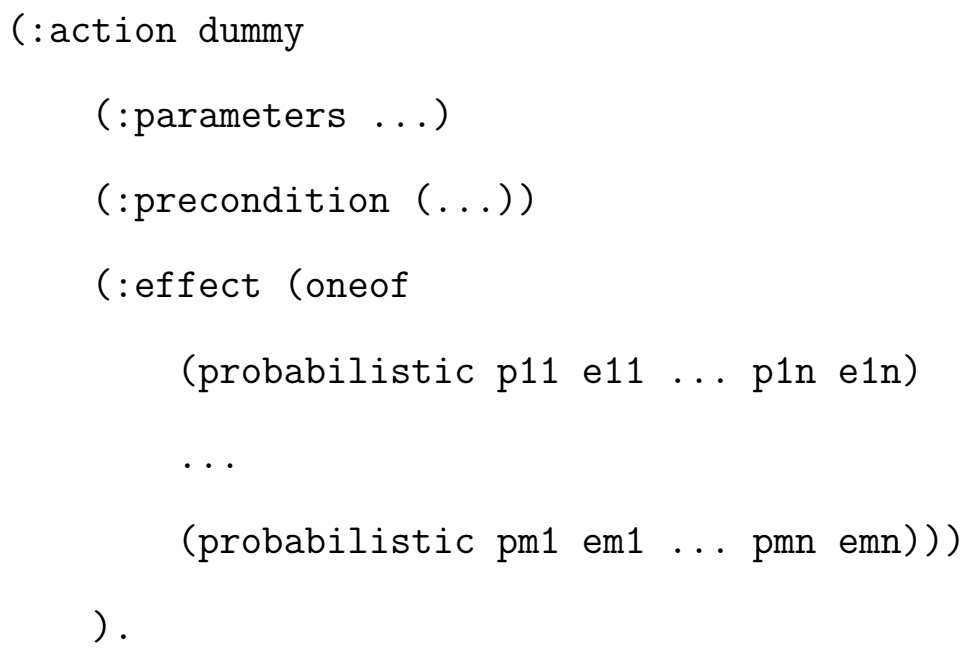

Naturalmente, a semântica dessa construção é a escolha aleatória sobre os diferentes operadores probabilísticos. Assim, a cada realização dessa ação, um dos efeitos probabilísticos é sorteado, e aplicado conforme a semântica usual da linguagem. Essa contrução obtém um MDPIP pois cada efeito probabilístico é uma distribuição de probabilidades, e o conjunto desses efeitos gera um conjunto de distribuições de probabilidades. 
Uma questão que poderia ser levantada é se novos encadeamentos de efeitos probabilísticos e não-determinísticos poderiam gerar formas ainda mais gerais de incertezas. A resposta é negativa. Qualquer nível de encadeamento de efeitos probabilísticos e nãodeterminísticos resulta, no máximo, em conjuntos de probabilidades. Para provar isso, note que efeitos probabilísticos induzirem distribuições de probabilidades, enquanto que efeitos não-determinísticos induzem mapas multivalorados (vide (DEMPSTER, 1967) ou (WALlEY, 1991, Seção 4.3.5, p. 182)). O encadeamento de efeitos da mesma natureza é trivial, que inclusive podem ser resumidos em um único efeito expandido. A utilização de efeitos não-determinísticos dentro de probabilísticos leva a uma função crença (WALLEY, 1991, Seção 5.13.2, p. 273), ou ainda a uma capacidade de Choquet de ordem infinita (TREVIZAN; COZMAN; DE BARROS, 2008). Efeitos probabilísticos dentro de efeitos nãodeterminísticos resultam em conjuntos de probabilidades, que sendo fechado e convexo leva a conjuntos credais (LEVI, 1980). Encadeamentos de ordens maiores podem ser analisadas desenvolvendo essas construções. 


\section{Programação Matemática para MDPIP}

Na Seção 2.4 foi feita uma revisão dos algoritmos existentes para MDPIP. Uma constatação feita ao analisar esses algoritmos é que não existiam abordagens baseadas em programação matemática para esse modelo. Note-se que Xu e Mannor (2007) desenvolveram um programa linear para lidar com MDP com parâmetros imprecisos, mas seus resultados se aplicaram apenas para o caso de recompensas incertas.

Acredita-se que programação matemática possa ser relevante para MDPIP da mesma forma que programação linear é para MDP. Em particular, acredita-se que possam ser empregados em MDP com múltiplos objetivos. Na literatura, esse problema é conhecido como Constrained MDP (MDP com restrições), pelo fato de cada função objetivo aparecer na formulação do problema como uma restrição adicional (PUTERMAN, 1994; ALTMAN, 1999).

Foram desenvolvidos três programas matemáticos para MDPIP: um programa binível, um programa bilinear e um programa multilinear. Esses resultados forma publicados no International Symposium on Imprecise Probabilities and Their Applications (ISIPTA) de 2007. Uma cópia do artigo publicado encontra-se no Apêndice I, e contém maiores detalhes técnicos e exemplos. 


\subsection{Programação Binível}

O programa linear (2.11) consiste na otimização da função valor $V(s)$ sujeito à Equação de Bellman sobre todos os possíveis pares estado-ação. Obtém-se, dessa forma, o menor valor de $V(s)$ que é maior ou igual do que todos os possíveis valores de $V(s, a)$, ou seja, igual ao valor máximo de $V(s, a)$. O problema para MDPIPs é basicamente o mesmo, porém com a forma modificada da Equação de Bellman (Equação (2.16), considerando o critério $\Gamma$-maximin). Assim, em analogia ao programa linear (2.11), pode-se escrever para MDPIPs:

$$
\begin{array}{ll}
\underset{V}{\operatorname{minimizar}} & \sum_{s} V(s) \\
\text { sujeito a } & V(s) \geq R(s, a)+\gamma \min _{P} \sum_{r} P(r \mid s, a) V(r),
\end{array}
$$

com óbvias restrições sobre as probabilidades $0 \geq P(r \mid s, a) \geq 1$ e $\sum_{r} P(r \mid s, a)=1, \forall s, a$. Note que a diferença para (2.11) consiste na otimização que agora consta na restrição do problema de otimização (5.1a). Isso caracteriza um problema de programação binível (VICENTE; CALAMAI, 1994).

Problemas de programação binível têm recebido um grande interesse na literatura, principalmente após 1980 (WHITE, 1993; VICENTE; CALAMAI, 1994), apesar dos primeiros trabalhos introduzindo o problema datarem da década de 1970 (VICENTE; CALAMAI, 1994; COLSON; SAVARD, 2007). Kolstad (1985) fornece uma das primeiras revisões sobre o tema, e desde então diversos trabalhos foram publicados com investigações atualizadas (e.g. (VICENTE; CALAMAI, 1994),(DEMPE, 2003),(COLSON; SAVARD, 2007)). Muitas vezes aparecem na literatura como a versão estática dos jogos de Stackelberg (EDMUNDS; BARD, 1991; WHITE, 1993), e possuem fortes conexões com problemas em economia. Problemas similares foram investigadas em conexão com programação linear, com óbvias aplicações para $\Gamma$-minimax (BEN-TAL; NEMIROVSKI, 1999; AVERBAKH, 2001; BEN-TAL; NEMIROVSKI, 2002).

Programas biníveis são, porém, geralmente não-convexos e não-diferenciáveis (COL- 
SON; SAVARD, 2007), e portanto intrinsicamente difíceis de resolver. Segundo Kolstad (1985), "infelizmente, bons métodos de solução para problemas binível não são geralmente disponíveis". Diversos algoritmos foram propostos para problemas dessa natureza (e.g. pontos extremos para o caso linear, branch and bound, pivotamento complementar, descent methods, penalty function e trust-region methods) (VICENTE; CALAMAI, 1994; COLSON; SAVARD, 2007), mas a complexidade é NP-Hard mesmo para o caso linear (VICENTE; CALAMAI, 1994; DEMPE, 2003; COLSON; SAVARD, 2007).

\subsection{Programação Bilinear}

A dificuldade de resolver o problema binível motivou a busca por uma forma mais simples de programa matemático para solução de MDPIPs. Uma simples manipulação do programa matemático permitiu a obtenção de um programa multilinear, apresentado a seguir:

$$
\begin{array}{ll}
\underset{V, P}{\operatorname{minimizar}} & \sum_{s} V(s) \\
\text { sujeito a } & V(s) \geq R(s, a)+\gamma \sum_{r} P(r \mid s, a) V(r) .
\end{array}
$$

A seguir, é provado que a solução do programa (5.2) é igual à solução do programa (5.1), e portanto, pode ser usado para resolver o MDPIP. Denota-se $\left(P_{B N}, V_{B N}\right)$ a solução do programa binível, e $\left(P_{B L}, V_{B L}\right)$ a solução do programa bilinear.

Teorema 1. $V_{B L}=V_{B N}$

Demonstração. Sejam $\Omega_{B N}$ e $\Omega_{B L}$ respectivamente os espaços de soluções para os Programas (5.1) e (5.2). Prova-se que $\Omega_{B N}$ é um subconjunto de $\Omega_{B L}$. Mostra-se então que nenhuma solução de $\Omega_{B L} \backslash \Omega_{B N}$ pode ter desempenho melhor que uma em $\Omega_{B N}$. Tem-se:

$$
\begin{array}{r}
\Omega_{B N}=\left\{(V, P): V \in \mathcal{V}, P=\underset{P \in \mathcal{P}}{\operatorname{argmin}} \sum_{r} P(r \mid s, a) V(r)\right\}, \\
\Omega_{B L}=\{(V, P): V \in \mathcal{V}, P \in \mathcal{P}\} .
\end{array}
$$

Dado que o espaço de soluções do segundo caso é o espaço inteiro $\mathcal{V} \times \mathcal{P}$, enquanto que 
no primeiro caso $P$ pode estar apenas no subespaço $\mathcal{V} \times \mathcal{P}_{B N}$ de $\mathcal{V} \times \mathcal{P}$, o Programa (5.2) produz uma função valor pelo menos tão baixo quanto o Programa (5.1). Então $V_{B L} \leq$ $V_{B N}$, pois $\Omega_{B L} \supset \Omega_{B N}$. Agora suponha que $V_{B L}<V_{B N}$. Para um estado $s \in \mathcal{S}, V_{B L}(s)=$ $R(s, a)+\gamma \sum_{r} P_{B L}(r \mid s, a) V_{B L}(r), \operatorname{com} P_{B L} \neq \operatorname{argmin}_{P} \sum_{r} P(r \mid s, a) V(r)$. Se tomarmos $P^{\prime}(r \mid s, a)=\operatorname{argmin}_{P} \sum_{r} P(r \mid s, a) V(r)$, então $V^{\prime}(s)=R(s, a)+\gamma \sum_{r} P^{\prime}(r \mid s, a) V_{B L}(r)<$ $V_{B L}(s)$, e $V_{B L}$ não é ótimo. Como $V_{B L}$ é ótimo (dado que ele considera todo o espaço $\mathcal{V} \times \mathcal{P}$ ), então $V_{B L} \nless V_{B N}$. Isso implica que $V_{B L}=V_{B N}$.

Um exemplo de aplicação desse algoritmo pode ser encontrado em Delgado et al. (2009), que utiliza o algoritmo acima em MDPIP fatorados.

\subsection{Programação Inteira}

Um resultado ainda mais interessante é obtido quando assume-se que os vértices do conjunto credal $K(r \mid s, a)$ são conhecidos. Considere a lista de vértices (cada vértice é uma distribuição sobre $\mathcal{S}$ ) para um conjunto credal $K(r \mid s, a),\left\{p_{1}, \ldots, p_{M}\right\}$. Cada distribuição nesse conjunto credal pode ser expressada como uma combinação convexa $\sum_{i=1}^{M} \alpha_{i} p_{i}$ em que $\alpha_{i} \geq 0$ e $\sum_{i} \alpha_{i}=1$. Pode-se escrever o objetivo como:

$$
\begin{array}{ll}
\underset{V, \alpha_{i, s, a}}{\operatorname{minimizar}} & \sum_{s} V(s) \\
\text { sujeito a } & V(s) \geq R(s, a)+\gamma \sum_{r} \sum_{i} \alpha_{i, s, a} p_{i}(r \mid s, a) V(r), \\
& \alpha_{i, s, a} \geq 0, \\
& \sum_{i} \alpha_{i, s, a}=1,
\end{array}
$$

em que indica-se explicitamente que $\alpha_{i, s, a}$ depende de $(s, a)$.

Agora utiliza-se o fato de que o máximo de um programa multilinear ocorre em um dos vértices do conjunto credal; portanto, necessariamente ocorre que $\alpha_{i, s, a} \in\{0,1\}$ na solução. Recorre-se então à seguinte transformação para, a partir do programa multilinear (5.3), produzir um programa inteiro, apenas assumindo que é possível limitar $V$ superior 
e inferiormente (tais limites podem ser produzidas usando os resultados de White III e El-Deib (1994)). Primeiro, $V(r) \in[l, u]$ é substituído por $l+(V(r)-l)$, e uma nova variável $\beta_{r}=V(r)-l \in[0, u-l]$ é criada. Cada $\alpha_{i, s, a} p_{i}(r \mid s, a) V(r)$ é então substituído por $\alpha_{i, s, a} p_{i}(r \mid s, a) l+\alpha_{i, s, a} p_{i}(r \mid s, a) \beta_{r}$. Note que $\alpha_{i, s, a} p_{i}(r \mid s, a) l$ é fácil de computar. Conforme $\alpha_{i, s, a}$ é restrito a 0 ou 1, pega-se cada termo $\alpha_{i, s, a} p_{i}(r \mid s, a) \beta_{r}$ e substitui-se $\alpha_{i, s, a} \beta_{r}$ por uma nova variável $\beta_{i, r, s, a}$. Para garantir que essa substituição não altera o problema original, as seguintes restrições lineares adicionais são introduzidas:

$$
\begin{aligned}
& 0 \leq \beta_{i, r, s, a} \leq \beta_{r}, \\
& \beta_{i, r, s, a} \leq \alpha_{i, s, a}(u-l), \\
& \beta_{r}-(u-l)+\alpha_{i, s, a}(u-l) \leq \beta_{i, r, s, a} .
\end{aligned}
$$

A primeira e a segunda restrições são óbvias (limitações de $\beta_{r}$ e $\alpha_{i, s, a}$. A última restrição implica o seguinte. Quando $\alpha_{i, s, a}=1, \beta_{r} \leq \beta_{i, r, s, a}$. Porém, como $\beta_{i, r, s, a} \leq \beta_{r}$ da primeira restrição, então podemos apenas ter que $\beta_{i, r, s, a}=\beta_{r}$, e podemos considerar o valor completo de $V(r)$. Se $\alpha_{i, s, a}=0$, então $\beta_{i, r, s, a} \leq 0$, mas $0 \leq \beta_{i, r, s, a}$ da primeira restrição, então $\beta_{i, r, s, a}=0$ e esse par estado-ação não é ótimo e não será considerado. 
Assim, o seguinte programa inteiro é obtido:

$$
\begin{array}{ll}
\underset{V, \alpha_{i, s, a}}{\operatorname{minimizar}} & \sum_{s} V(s) \\
\text { sujeito a } & V(s) \geq R(s, a)+\gamma \sum_{r} \sum_{i} \alpha_{i, s, a} p_{i}(r \mid s, a) V(r), \\
& \alpha_{i, s, a} \geq 0, \\
& \sum_{i} \alpha_{i, s, a}=1, \\
& \beta_{r}=V(r)-l, \\
& 0 \leq \beta_{r} \leq u-l, \\
& 0 \leq \beta_{i, r, s, a} \leq \beta_{r}, \\
& \beta_{i, r, s, a} \leq \alpha_{i, s, a}(u-l), \\
& \beta_{r}-(u-l)+\alpha_{i, s, a}(u-l) \leq \beta_{i, r, s, a} .
\end{array}
$$

Alguns exemplos de aplicação desses algoritmos podem ser encontrados em Shirota Filho et al. (2007), que encontra-se anexo no Apêndice I. 


\section{RTDP com Probabilidades Imprecisas}

RTDP pode ser adaptado para probabilidades imprecisas de forma simples. O procedimento apresentado a seguir é bastante semelhante ao utilizado por Trevizan, Cozman e de Barros (2008) para adaptar RTDP para MDPST. Alguns resultados são emprestados de Buffet e Aberdeen (2005), que analisaram o emprego de RTDP para resolver BMDP, que é forma especial de MDPIP em que as probabilidades são especificadas por intervalos. Entretanto, o algoritmo resultante é diferente pois Buffet e Aberdeen empregam uma técnica de ordenação de transições exibidas por BMDP que não são aplicáveis para MDPIP.

O primeiro passo para a adaptação de RTDP para problemas com probabilidades imprecisas é a modificação do Q-value (Eq. (2.12)). Essa modificação se assemelha àquela feita na equação de Bellman para MDPIP, e adota o critério pessimista para o comportamento da natureza:

$$
Q(s, a)=C(s, a)+\gamma \min _{P} \sum_{r \in \mathcal{S}} P(r \mid s, a) h(r) .
$$

Sob o critério $\Gamma$-maximin, esse Q-value pode ser usado para fazer a atualização gulosa da estimativa $h_{t}$ em um MDPIP da mesma forma que o Q-value original fazia para um MDP. Assim, basta substituir essa expressão no lugar da Eq. (2.12) no algoritmo Trial-based MDP.

O problema passa a ser então o ajuste das condições para que a convergência do algoritmo. Foi visto que para um MDP as seguintes condições 
1. o custo inicial de cada estado meta é zero,

2. existe pelo menos uma política própria,

3. todos os custos imediatos das transições partindo de um estado que não seja meta são positivos, e

4. o custo inicial de todos os estados não superestimam seu custo real,

devem ser satisfeitas. O único desses pontos que é mais complicado é o item 2. Segundo Barto, Bradtke e Singh (1995), uma política é própria se ela implica numa probabilidade não-nula de eventualmente atingir um estado meta a partir de qualquer estado inicial. Dessa forma, ao se adotar uma política própria, o estado meta será alcançado eventualmente de qualquer estado com probabilidade 1. Isso é o que Trevizan, Cozman e de Barros (2008) chamam de hipótese de alcançabilidade.

Uma forma de garantir a convergência do algoritmo é, então, respeitar essa hipótese. Esse foi o caminho adotado por Trevizan, Cozman e de Barros (2008). Uma forma mais simples é garantir que o algoritmo nunca deixe de visitar um estado, que segundo Barto, Bradtke e Singh (1995), é a única condição necessária para a convergência do RTDP. Essa é a motivação do Trial-based RTDP, e o caminho seguido por Buffet e Aberdeen (2005).

O problema passa então a ser garantir que nenhum estado deixe de ser visitado em múltiplas execuções do Trial-based RTDP. Como cada nova execução do algoritmo é iniciada em um novo estado, isso não necessariamente requer que todos os estados sejam alcançáveis do estado inicial. Basta que os estados sejam visitados em algum momento, ou seja, exista uma probabilidade não nula de partir de algum estado e chegar em cada um dos estados do sistema. Em BMDP, Buffet e Aberdeen (2005) mostraram que evitar as bordas do politopo determinado pelos intervalos de probabilidades é o suficiente para garantir essa condição. Isso porque é apenas nas bordas que a probabilidade de transição para algum estado pode ser zero (e portanto, esse estado deixaria de ser visitado pelo algoritmo). Para MDPIP, o mesmo raciocínio se aplica. Porém, o politopo é determinado 
pelo fecho convexo dos pontos do conjunto de probabilidade. Assim, evitando as bordas (ou seja, os extremos) desse politopo garante-se nenhuma probabilidade de transição é nula e que o algoritmo Trial-based RTDP com probabilidades imprecisas converge.

\subsection{Algoritmo RTPD Modificado}

A seguir, apresentamos o pseudo-código do algoritmo RTDP, baseado na implementação de Bonet e Geffner (2003). O Algoritmo 1 apresenta as rotinas básicas de chamada. Como o interesse é no Trial-based RTDP, a rotina RTDP realiza as seguidas chamadas do RTDPTRIAL.

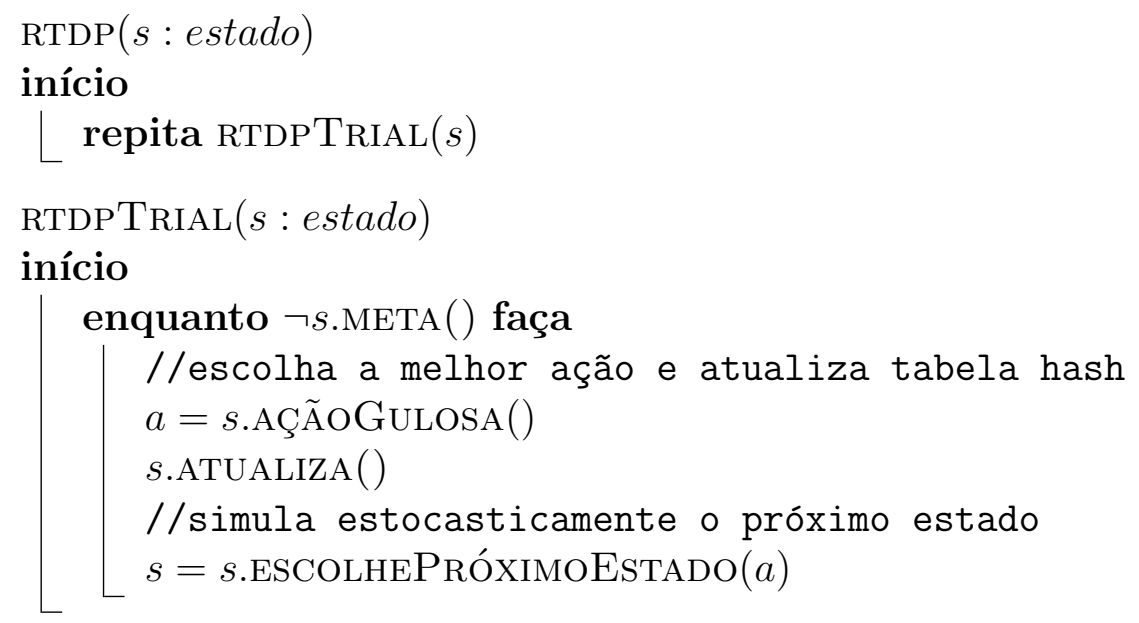

Algoritmo 1: Algoritmo RTDP, segundo Bonet e Geffner (2003)

No Algoritmo 2, o pseudo-código de algumas rotinas auxiliares são apresentadas. Note que o Q-value está adaptado para MDPIP, e que a escolha do próximo estado evita as bordas do conjunto credal.

No Algoritmo 3, apresenta-se o código para a rotina CHECKSolved. Essa rotina é utilizada no LRTDP para verificar se a utilidade do estado já convergiu, e portanto, pode ser marcado como resolvido.

Por fim, o Algoritmo 4 apresenta o código para o LRTDP, que é o RTDP com marcadores que utiliza o CHECKSOLVED para marcar estados resolvidos e assim, evitar atualizá-los de novo (BONET; GEFFNER, 2003). Com isso, ganha-se em eficiência e tempo de execução. 


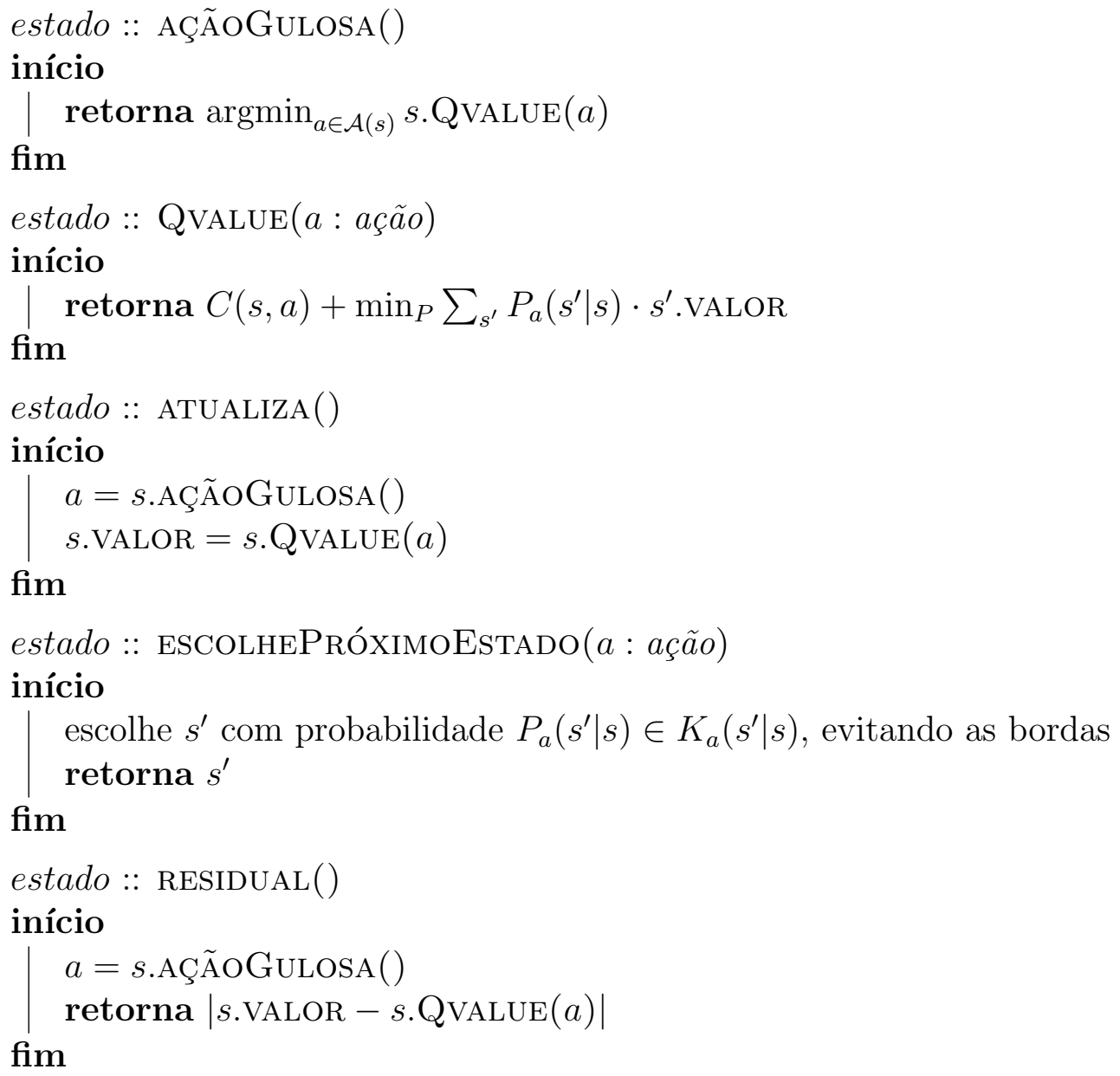

Algoritmo 2: Rotinas adicionais, segundo Bonet e Geffner (2003) 


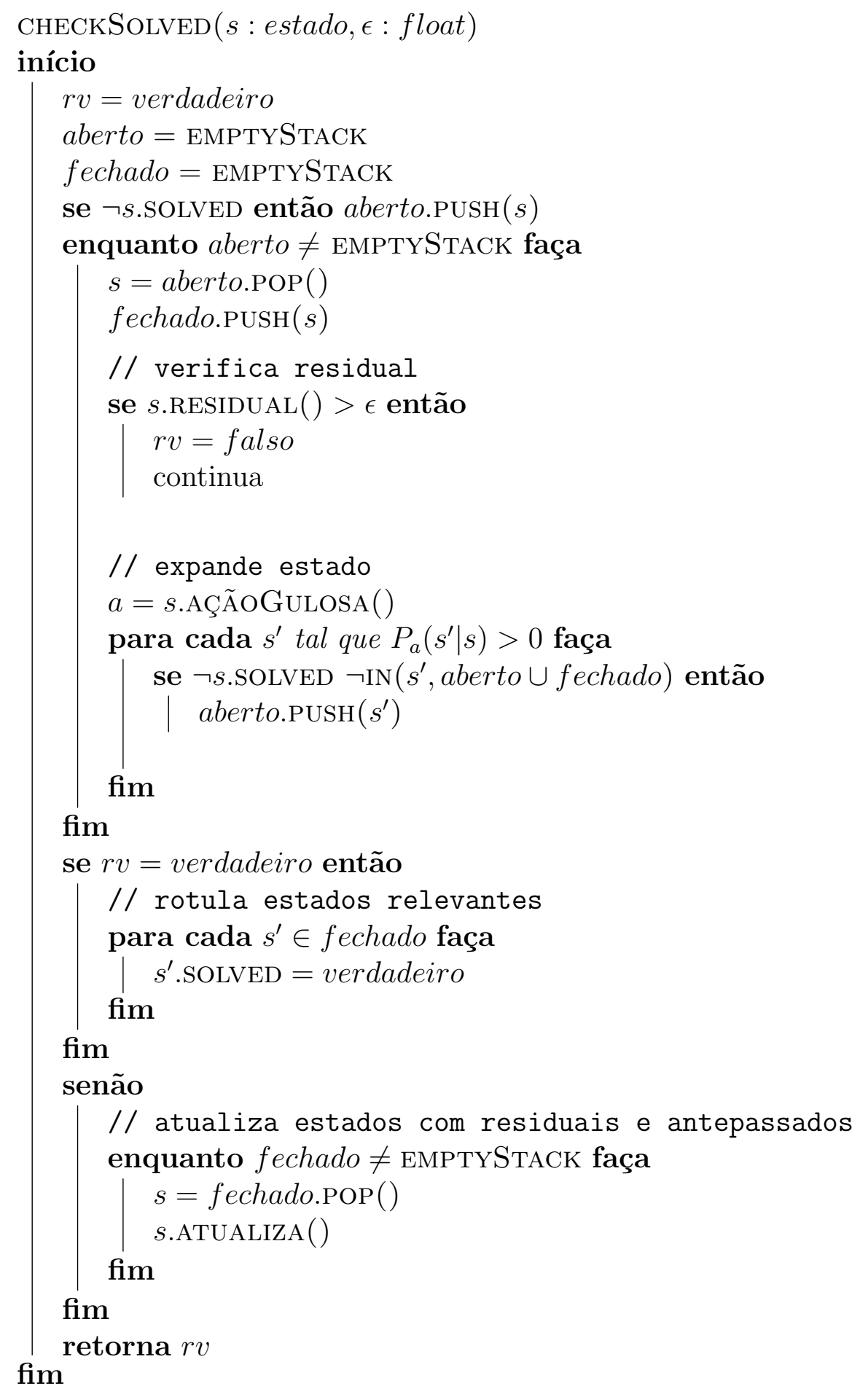

Algoritmo 3: checkSolved, segundo Bonet e Geffner (2003) 


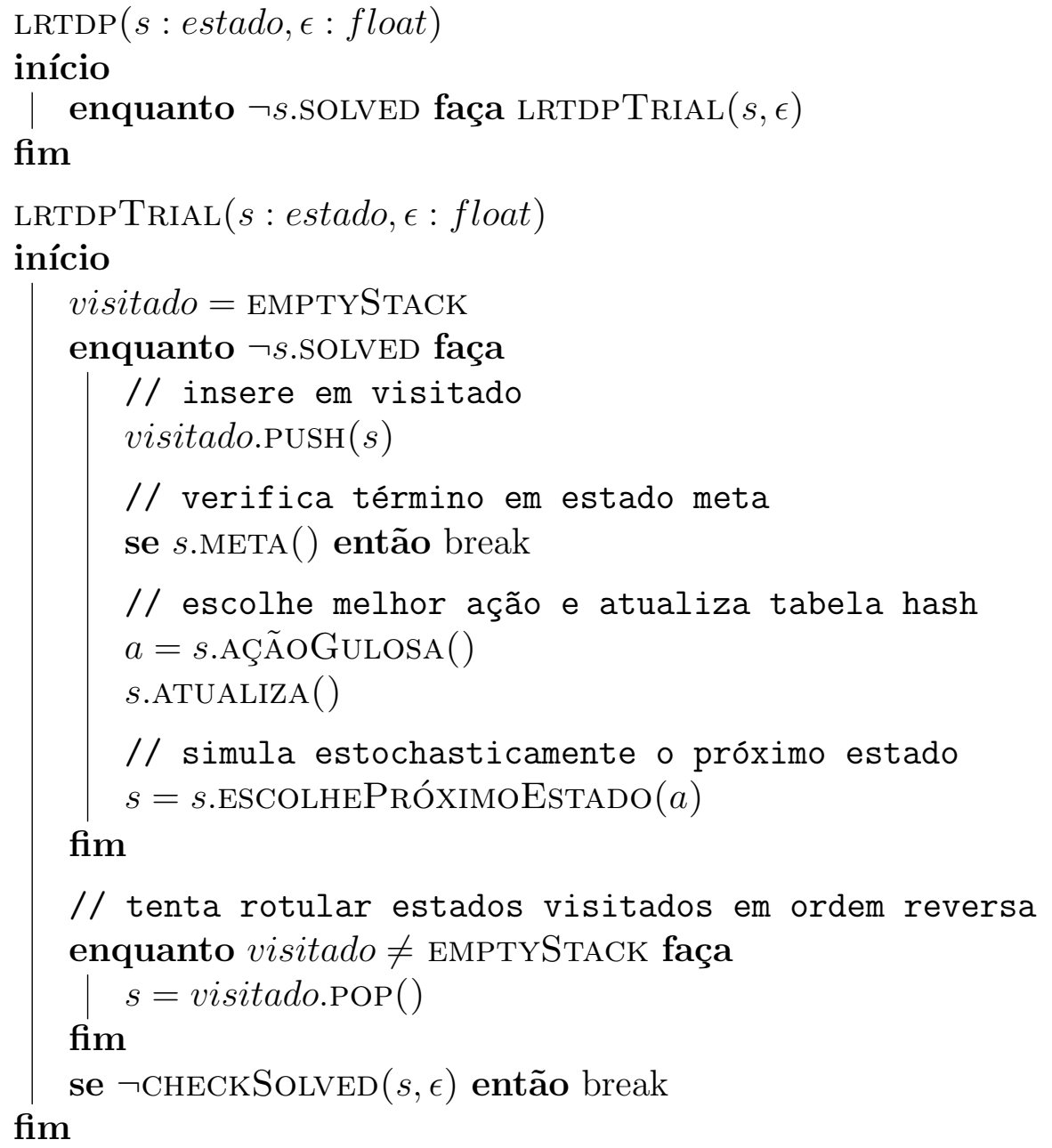

Algoritmo 4: Algoritmo LRTDP, segundo Bonet e Geffner (2003) 


\section{Trabalhos Futuros}

Durante o desenvolvimento dessa pesquisa, inúmeros assuntos foram abordados e diversas idéias foram desenvolvidas, algumas com sucesso, e outras não. No entanto, não deixam de ser idéias interessantes, que com algum esforço adicional possam chegar a resultados expressivos. Infelizmente o tempo é um recurso escasso, e não foi possível dar a devida atenção para cada uma. A seguir, listam-se algumas idéias com potencial em estudos futuros.

Constrained MDPIP Constrained MDP, ou MDPs com restrições, são resultado da existência de múltiplos objetivos em um problema modelado por um MDP. Apesar de programação dinâmica ter sido desenvolvida para esses problemas, programação linear tem sido a forma padrão para obter soluções. Especula-se que os programas matemáticos desenvolvidos neste trabalho possam ser relevantes para MDPIPs com restrições.

First-order MDPIP A abordagem utilizada neste trabalho não explora todo o potencial da linguagem de descrição do problema. O algoritmo RTDP trabalha com estados enumerativos, e a descrição relacional/fatorada em PPDDL precisa ser parseada, ou seja, interpretada e traduzida para uma estrutura adequada ao algoritmo. Mais sensato seria explorar esse potencial e elaborar formas de lidar com essas descrições. Algumas tentativas nesse sentido foram os trabalhos de Boutilier, Hanks e Dean (1999), Boutilier, Dearden e Goldszmidt (2000), Guestrin et al. (2003), Patrascu (2004) em MDPs fatorados, Boutilier, Reiter e Price (2001) em First-order MDP, Guestrin et al. (2003) em Relational MDP e Kersting e Raedt (2003) em Logical MDP. Mais recentemente, Delgado (2010) 
obteve uma formulação baseada no trabalho de Patrascu (2004) utilizando diagramas de decisão algebraicos parametrizados e probabilidades imprecisas com relativo sucesso. Porém, o desempenho mostra-se dependente da escolha de umas funções base, cuja seleção pode não ser trival na prática.

\section{Aplicações dos métodos desenvolvidos em problemas reais Aplicações são} sempre importantes para desmonstar a viabilidade do método desenvolvido. Além disso, apesar de ser um modelo recorrente na literatura, muitos poucos trabalho apresentam aplicações reais de MDPIP. Assim, seria interessante perseguir problemas em que o emprego de probabilidades imprecisas tornam-se fundamentais para a obtenção de uma solução.

Critérios de preferência parcial Preferências parciais possuem um problema que limitam sua aplicação em problemas reais: elas fornecem uma maneira de eliminar alternativas ruins, mas frequentemente esbarram em indecisão e tornam-se incapazes de escolher uma alternativa dentro um conjunto de opções admissíveis. Acredita-se que esse seja um dos grandes motivos da popularidade do $\Gamma$-maximin, que nunca falha em escolher uma alternativa. No entanto, é possível que se possa utilizar esses critérios de forma mais eficiente em conjunto com abordagens Bayesianas para MDPIP, de forma similar àquela adotada em Cozman e Krotov (1996).

\footnotetext{
Abordagem Bayesiana A abordagem Bayesiana é pouco explorada na literatura, mas é uma alternativa sensata para casos em que as probabilidades não são bem conhecidas. É necessário desenvolver algoritmos modernos, e identificar casos em que essa abordagem mostra-se vantajosa. Também sugere-se investigar o modelo Dirichlet impreciso (Imprecise Dirichlet Model) (BERNARD, 2005; WALLEY, 1996) em MDPIPs, que generaliza o modelo multinomial beta de Silver (1963).
}

Fundamentos Pelos motivos apresentados no Capítulo 3, uma maior investigação dos fundamentos de decisão sequencial com o uso de probabilidades imprecisas torna-se 
necessária. Sem a resposta a alguns desses pontos, é difícil confiar plenamente nos métodos atualmente empregados (e.g. inconsistência dinâmica de minimax). 


\section{Conclusões}

Neste trabalho, investigou-se o uso de processos de decisão markovianos com probabilidades imprecisas em problemas de planejamento. Processos de decisão markovianos são atualmente considerados o modelo padrão para planejamento probabilístico, e adotam um modelo de incertezas baseadas na teoria probabilística tradicional. Um problema dessa formulação é que especificações precisas de probabilidades, exigidas pela teoria clássica, muitas vezes não estão disponíveis e mostram-se difíceis ou inviáveis de obter.

O modelo impreciso relaxa essa suposição ao permitir que as probabilidades (e portanto, as incertezas) sejam especificadas de forma imprecisa, ou seja, através de conjuntos de valores. A precisão das atribuições refletem a quantidade de informação existente sobre o evento, e quanto mais informação estiver disponível, melhor e mais precisos podem ser as especificações. Naturalmente, especificações menos precisas levam a resultado de pior qualidade.

Pode-se considerar como três os resultados apresentados neste trabalho. Primeiro, obtiveram-se três algoritmos baseados em programação matemática para MDPIP. Apesar de ser considerada uma opção geralmente pouco eficiente frente às abordagens de programação dinâmica, programação matemática consiste em uma formulação elegante para o problema e mostra-se interessante em diversas situações. Em particular, espera-se que sejam relevantes para problemas multi-objetivos com probabilidades imprecisas, uma vez que problemas multi-objetivos clássicos (que utilizam o modelo probabilístico tradicional) são geralmente tratados através de programação linear.

Em um segundo momento, foi investigada a aplicação do modelo impreciso em pro- 
blemas de planejamento. Aproveitando-se os resultados obtidos por Trevizan, Cozman e de Barros (2008) para MDPST, obteve-se uma adaptação do algoritmo RTDP para problemas com probabilidades imprecisas. A descrição do problema foi feita na extensão de PPDDL inicialmente proposta por Trevizan, Cozman e de Barros (2008), que pode apenas ser captada através de um MDPIP. Mostramos como o encadeamento de efeitos probabilísticos e não-determinísticos levam a conjuntos de probabilidades, e provamos que futuros encadeamentos não alteram a expressividade da linguagem. Dessa forma, em termos de efeitos em PPDDL, um MDPIP é suficiente.

Por fim, apresentou-se uma discussão sobre o uso de probabilidades imprecisas e tomada de decisão sequencial. Provavelmente, esse é o ponto mais importante do trabalho, pois ressalta a necessidade de se desenvolver alguns aspectos dos fundamentos antes de aplicar esse modelo de incerteza ao âmbito de decisões sequencias. Em particular, notase que o critério $\Gamma$-minimax, bastante utilizado em estatística robusta e onipresente em MDPIP, foi mostrado ser dinamicamente inconsistente em decisões sequenciais. O que não está claro é se essa inconsistência também se aplica a MDPIP, ou se esse modelo exibe alguma propriedade que a protege contra essa situação. Na verdade essa não é uma questão específica a MDPs ou MDPIPs, mas que se aplica a toda teoria de decisão que utilize probabilidades imprecisas. 


\section{Referências Bibliográficas}

ALTMAN, E. Denumerable constrained Markov decision processes and finite approximations. Mathematics of Operations Research, v. 19, n. 1, p. 169-191, 1994.

ALTMAN, E. Constrained Markov decision processes. Boca Raton, Florida: Chapman \& Hall, 1999.

AVERBAKH, I. On the complexity of a class of combinatorial optimization problems with uncertainty. Mathematical Programming, v. 90, n. 2, p. 263-272, 2001.

BACCHUS, F. The power of modeling-a response to PDDL2.1. Journal of Artificial Intelligence Research, v. 20, p. 125-132, 2003.

BAGNELL, J. A.; NG, A. Y.; SCHNEIDER, J. G. Solving uncertain Markov decision processes. Pittsburgh PA, 2001.

BARRETT, A. et al. UCPOP user's manual. Seattle, WA, Novembro 1995.

BARTO, A. G.; BRADTKE, S. J.; SINGH, S. P. Learning to act using real-time dynamic programming. Artificial Intelligence, v. 72, n. 1-2, p. 81-138, 1995.

BELLMAN, R. A Markovian decision process. Journal of Mathematics and Mechanics, v. 6, n. 4, p. 679-684, 1957.

BELLMAN, R. Dynamic programming. Mineola, Nova York: Dover Publications, Inc., 2003.

BEN-TAL, A.; NEMIROVSKI, A. Robust solutions of uncertain linear programs. Operations Research Letters, v. 25, n. 1, p. 1-13, 1999.

BEN-TAL, A.; NEMIROVSKI, A. Robust optimization - methodology and applications. Mathematical Programming, v. 92, n. 1, p. 453-480, 2002.

BERGER, J. O. Statistical Decision Theory and Bayesian Analysis. EUA: SpringerVerlag, 1985.

BERNARD, J.-M. An introduction to the imprecise Dirichlet model for multinomial data. International Journal of Approximate Reasoning, v. 39, n. 2-3, p. 123-150, 2005.

BERTSEKAS, D. P. Distributed dynamic programming. IEEE Transactions on Automatic Control, v. 27, n. 3, p. 610-616, 1982.

BERTSEKAS, D. P.; TSITSIKLIS, J. N. An analysis of stochastic shortest path problems. Mathematics of Operations Research, v. 16, n. 3, p. 580-595, 1991. 
BODDY, M. Imperfect match: PDDL 2.1 and real applications. Journal of Artificial Intelligence Research, v. 20, p. 133-137, 2003.

BONET, B.; GEFFNER, H. GPT: a tool for planning with uncertainty and partial information. Em: CIMATTI, A. et al. (Ed.). Proc. IJCAI-01 Workshop on Planning with Uncertainty and Partial Information. Seattle, WA: Morgan Kaufmann, 2001. p. 82-87.

BONET, B.; GEFFNER, H. Planning and control in artificial intelligence: A unifying perspective. Applied Intelligence, v. 14, p. 237-252, 2001.

BONET, B.; GEFFNER, H. Solving Stochastic Shortest-Path Problems with RTDP. Venezuela, 2002.

BONET, B.; GEFFNER, H. Labeled RTDP: Improving the convergence of real-time dynamic programming. Em: GIUNCHIGLIA, E.; MUSCETTOLA, N.; NAU, D. (Ed.). ICAPS 2003: Proceedings of the Thirteenth International Conference on Automated Planning and Scheduling. Menlo Park, California: AAAI Press, 2003. p. 12-21.

BONET, B.; GIVAN, R. 5th international planning competition: Non-deterministic track - call for participation. IPC-2005. 2005.

BOUTILIER, C.; DEARDEN, R.; GOLDSZMIDT, M. Stochastic dynamic programming with factored representations. Artificial Intelligence, v. 121, n. 1-2, p. 49-107, 2000.

BOUTILIER, C.; HANKS, S.; DEAN, T. Decision-theoretic planning: Structural assumptions and computational leverage. Journal of Artificial Intelligence Research, v. 11, p. 1-94, 1999.

BOUTILIER, C.; REITER, R.; PRICE, B. Symbolic dynamic programming for first-order MDPs. Em: NEBEL, B. (Ed.). IJCAI-01: Proceedings of the Seventeenth International Joint Conference on Artificial Intelligence. São Francisco, CA: Morgan Kaufmann, 2001. p. 690-700.

BUFFET, O.; ABERDEEN, D. Robust planning with (L)RTDP. Em: KAELBLING, L. P.; SAFFIOTTI, A. (Ed.). IJCAI-05: Proceeding of the Nineteenth International Joint Conference on Artificial Intelligence. Edinburgh, Scotland: Professional Book Center, 2005. p. 1214-1219.

CIMATTI, A.; ROVERI, M.; TRAVERSO, P. Strong planning in non-deterministic domains via model checking. Em: AIPS 1998 Proceedings. Pittsburgh, EUA: AAAI, 1998. p. 36-43.

COLSON, P. M. B.; SAVARD, G. An overview of bilevel optimization. Annals of Operations Research, v. 153, n. 1, p. 235-256, 2007.

COOMAN, G. de; TROFFAES, M. C. M. Dynamic programming for deterministic discrete-time systems with uncertain gain. International Journal Approximate Reasoning, v. 39, n. 2-3, p. 257-278, 2005.

COZMAN, F.; KROTOV, E. Quasi-bayesian strategies for efficient plan generation: Application to the plan to observe problem. Em: ASSOCIATION FOR UNCERTAINTY IN ARTIFICIAL INTELLIGENCE. Proceedings of the 12th Annual Conference on Uncertainty in Artificial Intelligence (UAI-96). San Francisco, CA: Morgan Kaufmann, 1996. p. 186-193. 
DELGADO, K. V. Processo de decisão Markoviano fatorado com probabilidades imprecisas. Tese (Doutorado) — Universidade de São Paulo, 2010.

DELGADO, K. V. et al. Representing and solving factored markov decision processes with imprecise probabilities. Em: AUGUSTIN, T. et al. (Ed.). Proceedings of the 6th International Symposium on Imprecise Probabilities: Theories and Applications. Durham, UK: Society for Imprecise Probability: Theories and Applications, 2009. p. 169-178.

DEMPE, S. Annotated bibliography on bilevel programming and mathematical programs with equilibrium constraints. Optimization, v. 52, n. 3, p. 333-359, 2003.

DEMPSTER, A. P. Upper and lower probabilities induced by a multivalued mapping. The Annals of Mathematical Statistics, v. 38, n. 2, p. 325-339, 1967.

DENARDO, E. V. Contraction mappings in the theory underlying dynamic programming. SIAM Review, v. 9, n. 2, p. 165-177, 1967.

D'EPENOUX, F. A probabilistic production and inventory problem. Management Science, v. 10, n. 1, p. 98-108, 1963.

EDELKAMP, S.; HOFFMANN, J. PDDL2.2: The Language for the Classical Part of the 4th International Planning Competition. Friburgo, Alemanha, Janeiro 2004.

EDMUNDS, T. A.; BARD, J. F. Algorithms for nonlinear bilevel mathematical programs. IEEE Transactions on Systems, Man and Cibernetics, v. 21, n. 1, p. 83-89, 1991.

FEINBERG, E. A.; SHWARTZ, A. (Ed.). Handbook of Markov decision processes: methods and applications. USA: Kluwer Academic Publishers, 2002. (International series in operations research and management sciences).

FOX, M.; LONG, D. PDDL2.1: An extension to PDDL for expressing temporal planning domains. Journal of Artificial Intelligence Research, v. 20, p. 61-124, 2003.

FRIEDMAN, N.; HALPERN, J. Plausibility measures: A user's guide. Em: Proceedings of the Eleventh Conference Annual Conference on Uncertainty in Artificial Intelligence (UAI-95). San Francisco, CA: Morgan Kaufmann, 1995. p. 175-184.

GEFFNER, H. Perspectives on artificial intelligence planning. Em: DECHTER, R.; KEARNS, M.; SUTTON, R. (Ed.). Proceedings of the Eighteenth National Conference on Artificial Intelligence. Edmonton, Alberta: AAAI Press, 2002. p. 1013-1023.

GEFFNER, H. PDDL 2.1: Representation vs. computation. Journal of Artificial Intelligence Research, v. 20, p. 139-144, 2003.

GEREVINI, A.; LONG, D. Plan Constraints and Preferences in PDDL3. Itália, Agosto 2005 .

GEREVINI, A.; LONG, D. Preferences and soft constraints in PDDL3. Em: Proceedings of the ICAPS 2006 Workshop on Preferences and Soft Constraints in Planning. Menlo Park, California: AAAI Press, 2006. p. 46-54. 
GIVAN, R.; LEACH, S. M.; DEAN, T. Bounded-parameter Markov decision processes. Artificial Intelligence, v. 122, n. 1-2, p. 71-109, 2000.

GUESTRIN, C. et al. Efficient solution algorithms for factored MDPs. Journal of Artificial Intelligence Research, v. 19, p. 399-468, 2003.

HANSSON, S. O. Decision Theory: A brief introduction. Estocolmo, Suécia, 2005.

HARMANEC, D. Generalizing Markov decision processes to imprecise probabilities. Journal of Statistical Planning and Inference, v. 105, p. 199-213, 2002.

HELMERT, M. PddlResources - IPC-2008, Deterministic Part. 2008. http: //ipc.informatik.uni-freiburg.de/PddlResources.

HORDIJK, A.; KALLENBERG, L. C. M. Constrained undiscounted stochastic dynamic programming. Mathematics of Operations Research, v. 9, n. 2, p. 276-289, 1984.

ITOH, H.; NAKAMURA, K. Partially observable Markov decision processes with imprecise parameters. Artificial Intelligence, v. 171, n. 8-9, p. 453-490, 2007.

IYENGAR, G. N. Robust dynamic programming. Mathematics of Operations Research, v. 30, n. 2, p. 257-280, 2005.

KALYANASUNDARAM, S.; CHONG, E. K. P.; SHROFF, N. B. Markov decision processes with uncertain transition rates: Sensitivity and robust control. Em: Proceedings of the 41st IEEE Conference on Decision and Control. Las Vegas, Nevada: IEEE, 2002. p. 3799-3804.

KERSTING, K.; RAEDT, L. D. Logical Markov decision programs. Em: IJCAI Workshop on Learning Statistical Models from Relational Data. [S.l.: s.n.], 2003. p. 63-70.

KIKUTI, D.; COZMAN, F. G.; CAMPOS, C. P. de. Partially ordered preferences in decision trees: computing strategies with imprecision in probabilities. Em: BRAFMAN, R.; JUNKER, U. (Ed.). IJCAI Workshop on Advances in Preference Handling. Edinburgo, Reino Unido: IJCAI, 2005. p. 118-123.

KIKUTI, D.; COZMAN, F. G.; SHIROTA FILHO, R. Sequential decision making with partially ordered preferences. Artificial Intelligence, v. 175, n. 7-8, p. 1346-1365, Maio 2011.

KOLSTAD, C. D. A Review of the Literature on Bi-level Mathematical Programming. Los Alamos, Novo Mexico, EUA, 1985.

KORF, R. E. Real-time heuristic search: First results. Em: FORBUS, K.; SHROBE, H. (Ed.). AAAI-87: Proceedings of the Sixth National Conference on Artificial Intelligence. Seattle, Washington: MIT Press, 1987. p. 133-138.

KORF, R. E. Real-time heuristic search: New results. Em: MITCHELL, T. M.; SMITH, R. G. (Ed.). AAAI-88: Proceedings of the Seventh National Conference on Artificial Intelligence. Saint Paul, Minnesota: MIT Press, 1988. p. 139-144.

KORF, R. E. Real-time heuristic search. Artificial Intelligence, v. 42, n. 2-3, p. 189-211, Março 1990. 
KURANO, M.; YASUDA, M.; NAKAGAMI, J. Interval methods for uncertain markov decision processes. Em: The International Workshop on Markov Processes and Controlled Markov Chains. Holanda: Kluwer Academic Publishers, 1999. p. 22-28.

LAVALLE, S. M. Planning Algorithms. New York, NY, USA: Cambridge University Press, 2006.

LEVI, I. The Enterprise of Knowledge: An Essay on Knowledge, Credal Probability, and Chance. Cambridge, Massachusetts: MIT Press, 1980.

MCDERMOTT, D. The 1998 AI planning systems competition. AI Magazine, v. 21, n. 2 , p. $35-55,2000$.

MCDERMOTT, D. PDDL 2.1 - The art of the possible? Commentary on Fox and Long. Journal of Artificial Intelligence Research, v. 20, p. 145-148, 2003.

MCDERMOTT, D. et al. PDDL - The Planning Domain Definition Language. New Haven, CT, Outubro 1998.

MCMAHAN, H. B.; LIKHACHEV, M.; GORDON, G. J. Bounded real-time dynamic programming: RTDP with monotone upper bounds and performance guarantees. Em: RAEDT, L. D.; WROBEL, S. (Ed.). ICML 2005: Proceedings of the 22nd International Conference on Machine Learning. USA: ACM Press, 2005. p. 569-576.

MONAHAN, G. E. A survey of partially observable Markov decision processes: Theory, models and algorithms. Management Science, v. 28, n. 1, p. 1-16, 1982.

NILIM, A.; GHAOUI, L. E. Robust control of Markov decision processes with uncertain transition matrices. Operations Research, v. 53, n. 5, p. 780-798, 2005.

PATRASCU, R.-E. Linear Approximations for Factored Markov Decision Processes. Tese (Doutorado) - Universidade de Waterloo, 2004.

PERNY, P.; SPANJAARD, O.; WENG, P. Algebraic Markov decison processes. Em: KAELBLING, L. P.; SAFFIOTTI, A. (Ed.). Proceedings of the Nineteenth International Joint Conferences on Artificial Intelligence - IJCAI'05. Edimburgo: Professional Book Center, 2005. p. 1326-1331.

PUTERMAN, M. L. Markov Decision Processes. New York: John Wiley and Sons, 1994. (Wiley series in probability and mathematical statistics).

ROSS, S.; CHAIB-DRAA, B.; PINEAU, J. Bayesian reinforcement learning in continuous POMDPs with application to robot navigation. Em: Proceedings of the 2008 IEEE International Conference on Robotics and Automation. Pasadena, CA: IEEE, 2008. p. $2845-2851$.

RUSSELL, S. J.; NORVIG, P. Artificial Intelligence: a Modern Approach. Terceira edição. Nova Jersey: Prentice Hall, 2010.

SANNER, S. Relational and first-order decision-theoretic planning: Foundations and future directions. Depth Oral Report. 2004.

SANNER, S. P. First-Order Decision-Theoretic Planning in Structured Relational Environments. Tese (Doutorado) — Universidade de Toronto, 2008. 
SATIA, J. K. Markovian decision process with uncertain transition matrices or/and probabilistic observation of states. Tese (Doutorado) — Stanford University, 1968.

SATIA, J. K.; LAVE JR., R. E. Markovian decision processes with uncertain transition probabilities. Operations Research, v. 21, n. 3, p. 728-740, 1973.

SAVAGE, L. J. The Foundations of Statistics. Second revised. New York: Dover Publications, Inc., 1954.

SCHWEITZER, P. J.; SEIDMANN, A. Generalized polynomial approximations in Markovian decision processes. Journal of Mathematical Analysis and Applications, v. 110, n. 2, p. 568-582, 1985.

SEIDENFELD, T. A constrast between two decision rules for use with (convex) sets of probabilities: Gamma-maximin versus E-admissibility. Synthese, v. 140, p. 69-88, 2004.

SEN, A. Rationality and uncertainty. Theory and Decision, v. 18, p. 109-127, 1985.

SHIROTA FILHO, R. et al. Multilinear and integer programming for Markov decision processes with imprecise probabilities. Em: COOMAN, G. de; VEJNAROVÁ, J.; ZAFFALON, M. (Ed.). ISIPTA'0\%: Proceedings of the Fifth International Symposium on Imprecise Probability: Theory and Applications. Praga, República Tcheca: Action M Agency, 2007. p. 395-404.

SILVER, E. A. Markovian decision processes with uncertain transition probabilities or rewards. Cambridge, MA, 1963.

SMITH, D. E. The case for durative actions: A commentary on PDDL2.1. Journal of Artificial Intelligence Research, v. 20, p. 149-154, 2003.

SMITH, T.; SIMMONS, R. Focused real-time dynamic programming for MDPs: Squeezing more out of a heuristic. Em: GIL, Y.; MOONEY, R. J. (Ed.). AAAI-06: Proceedings of the Twenty-First National Conference on Artificial Intelligence. Boston, MA: AAAI Press, 2006. p. 1227-1232.

TREVIZAN, F. W.; COZMAN, F. G.; DE BARROS, L. N. Planning under risk and Knightian uncertainty. Em: VELOSO, M. M. (Ed.). IJCAI-0\%: Proceedings of the 20th International Joint Conference on Artificial Intelligence. São Francisco, CA, EUA: Morgan Kaufmann Publishers Inc., 2007. p. 2023-2028.

TREVIZAN, F. W.; COZMAN, F. G.; DE BARROS, L. N. Mixed probabilistic and nondeterministic factored planning through Markov decision processes with set-valued transitions. Em: ICAPS Workshop: A Reality Check for Planning and Scheduling Under Uncertainty. Menlo Park, Califórnia: AAAI Press, 2008.

TROFFAES, M. C. M.; HUNTLEY, N.; SHIROTA FILHO, R. Sequential decision processes under act-state independence with arbritrary choice functions. Em:

HüLLERMEIER, E.; KRUSE, R.; HOFFMANN, F. (Ed.). Information Processing and Management of Uncertainty in Knowledge-Based Systems. Theory and Methods - 13th International Conference, IPMU 2010, Dortmund, Alemanha. Proceedings, Parte I. Dortmund, Alemanha: Springer, 2010. (Communications in Computer and Information Science, v. 80), p. 98-107. 
TROFFAES, M. M. C. Decision making under uncertainty using imprecise probabilities. International Journal of Approximate Reasoning, v. 45, n. 1, p. 17-29, 2007.

UTKIN, L. V.; AUGUSTIN, T. Powerful algorithms for decision making under partial prior information and general ambiguity attitudes. Em: COZMAN, F. G.; NAU, R.; SEIDENFELD, T. (Ed.). ISIPTA'05: Proceedings of the Fourth International Symposium on Imprecise Probabilities and Their Applications. Pittsburgh, PA: SIPTA, 2005. p. 359-368.

VICENTE, L. N.; CALAMAI, P. H. Bilevel and multilevel programming: A bibliography review. Journal of Global Optimization, v. 5, n. 3, p. 291-306, 1994.

VON NEUMANN, J.; MORGENSTERN, O. Theory of Games and Economic Behavior. Third. Princeton, NJ: Princeton University Press, 1953.

WALLEY, P. Statistical Reasoning with Imprecise Probabilities. London: Chapman and Hall, 1991.

WALLEY, P. Inferences from multinomial data: Learning about a bag of marbles. Journal of the Royal Statistics Society. Series B (Methodological), v. 58, n. 1, p. 3-57, 1996.

WHITE, D. J. Real applications of Markov decision processes. Interfaces, v. 15, n. 6, p. 73-83, Nov.-Dec. 1985.

WHITE, D. J. Further real applications of Markov decision processes. Interfaces, v. 18, n. 5, p. 55-61, Sep.-Oct. 1988.

WHITE, D. J. Markov Decision Processes. England: John Wiley and Sons, 1993.

WHITE III, C. C.; EL-DEIB, H. K. Markov decision processes with imprecise transition probabilities. Operations Research, v. 42, n. 4, p. 739-749, Julho-Agosto 1994.

XU, H.; MANNOR, S. The robustness-performance tradeoff in Markov decision processes. Em: SCHÖLKOPF, B.; PLATT, J.; HOFFMAN, T. (Ed.). Advances in Neural Information Processing Systems 19. Cambridge, MA: MIT Press, 2007. p. $1537-1544$

YOUNES, H. L. S.; LITTMAN, M. L. PPDDL1.0: An Extension to PDDL for Expressing Planning Domains with Probabilistic Effects. Pittsburgh, PA, 2004.

ZHAO, J.; SUN, J.; YIN, M. Recent advances in conformant planning. Em: Proceedings of the 2007 IEEE International Conference on Robotics and Biomimetics. Sanya, China: IEEE, 2007. p. 2001-2006. 
Apêndice I - Artigo: ISIPTA 2007 


\title{
Multilinear and Integer Programming for Markov Decision Processes with Imprecise Probabilities
}

\author{
Ricardo Shirota Filho \\ Escola Politécnica, \\ Universidade de São Paulo, SP, Brazil \\ ricardo.shirota@poli.usp.br \\ Felipe Werndl Trevizan \\ Departamento de Tecnología, \\ Universitat Pompeu Fabra, Barcelona, Spain \\ felipe.trevizan@upf.edu
}

Fabio Gagliardi Cozman

Escola Politécnica,

Universidade de São Paulo, SP, Brazil

fgcozman@usp.br

Cassio Polpo de Campos

Escola de Artes, Ciências e Humanidades

Universidade de São Paulo, SP, Brazil cassiopc@usp.br

\author{
Leliane Nunes de Barros \\ Instituto de Matemática e Estatística \\ Universidade de São Paulo, SP, Brazil \\ leliane@ime.usp.br
}

\begin{abstract}
Markov Decision Processes (MDPs) are extensively used to encode sequences of decisions with probabilistic effects. Markov Decision Processes with Imprecise Probabilities (MDPIPs) encode sequences of decisions whose effects are modeled using sets of probability distributions. In this paper we examine the computation of $\Gamma$-maximin policies for MDPIPs using multilinear and integer programming. We discuss the application of our algorithms to "factored" models and to a recent proposal, Markov Decision Processes with Set-valued Transitions (MDPSTs), that unifies the fields of probabilistic and "nondeterministic" planning in artificial intelligence research.
\end{abstract}

Keywords. Markov Decision Processes with Imprecise Probabilities, $\Gamma$-maximin criterion, multilinear and integer programming.

\section{Introduction}

In this paper we are concerned with the computation of policies, or plans, that aim at maximizing reward over a possibly countably infinite sequence of stages. At each stage, our decision maker finds herself in a state and she must select an action. As a result of this decision, she gets a reward, and she moves to a new state. The process is then repeated. We focus on situations where transitions between states are modeled by credal sets; that is, by sets of probability distributions. Thus we focus on Markov Decision Processes with Imprecise Probabilities (MDPIPs), following a sizeable literature that has steadily grown in the last few decades. We review the basic concepts on MDPIPs in Section 2; we offer a relatively long review as we attempt to capture, in a somewhat organized form, various concepts dispersed in the literature.

There are several possible criteria that we might use to evaluate policies in an MDPIP. The term optimal pol$i c y$ is used in this paper in connection with $\Gamma$-maximin expected total discounted reward; that is, highest expected total discounted reward under the worst possible selection of probabilities.

We show how to reduce the generation of optimal policies for an MDPIP to multilinear/integer programming in Section 3. We also discuss in that section the practical reasons to pursue such a programming solution. We comment on the relationship between multilinear programming and "factored" models in Section 4. We then move, in Section 5, to a recently proposed special type of MDPIP that has particularly pleasant properties and important applications, the Markov Decision Process with Set-valued Transitions (MDPSTs).

\section{Background}

In this section we review basic facts about MDPs, MDPIPs, evaluation criteria, and algorithms.

\subsection{MDPs}

Markov Decision Processes (MDPs) are used in many fields to encode possibly infinite sequences of decisions under uncertainty. For historical review, basic technical development, and substantial reference to related 
literature, the reader may consult books by Puterman [29] and Bertsekas [5]. In this paper we consider MDPs that are described by:

- a countable set $\mathcal{T}$ of stages; a decision is made at each stage.

- a finite set $\mathcal{S}$ of states.

- a finite set of actions $\mathcal{A}$; the set of actions may be indexed by states, but we simplify notation here by assuming a single set of actions for all states.

- a conditional probability distribution $P_{t}$ that specifies the probability of transition from state $s$ to state $r$ given action $a$ at stage $t$. We assume that probabilities are stationary (do no depend on $t$ ) and write $P(r \mid s, a)$.

- a reward function $R_{t}$ that indicates how much is gained (or lost, by using a negative value) when action $a$ is selected in state $s$ at stage $t$. We assume the reward function to be stationary and write $R(s, a)$.

We refer to the state obtained at stage $t$, in a particular realization of the process, as $s_{t}$; likewise, the action selected at stage $t$ is referred to as $a_{t}$.

The history $h_{t}$ of an MDP at stage $t$ is the sequence of states and actions visited by the process, $\left[s_{1}, a_{1}, \ldots, a_{t-1}, s_{t}\right]$. The Markov assumption that is adopted for MDPs is that $P\left(s_{t} \mid h_{t-1}, a_{t}\right)=$ $P\left(s_{t} \mid s_{t-1}, a_{t}\right)$; consequently:

$$
\begin{aligned}
P\left(h_{t} \mid s_{1}\right)= & P\left(s_{t} \mid s_{t-1}, a_{t-1}\right) P\left(s_{t-1} \mid s_{t-2}, a_{t-2}\right) \\
& \ldots \times P\left(s_{3} \mid s_{2}, a_{2}\right) P\left(s_{2} \mid s_{1}, a_{1}\right) .
\end{aligned}
$$

A decision rule $d_{t}(s, t)$ indicates the action that is to be taken in state $s$ at stage $t$. A policy $\pi$ is a sequence of decision rules, one for each stage. A policy may be deterministic or randomized; that is, it may prescribe actions with certainty, or rather it may just prescribe a probability distribution over the actions. A policy may also be history-dependent or not; that is, it may depend on all states and actions visited in previous stages, or just on the current state. A policy that is not history-dependent is called Markovian. A Markovian policy induces a probability distribution over histories through Expression (1).

We also assume that an MDP with infinite horizon (that is, with infinite $\mathcal{T}$ ) may always stop with some probability. In fact, we assume that the process stops with geometric probability: the process stops at stage $t$ with probability $(1-\gamma) \gamma^{t-1}$ (independently of all other aspects of the process). Then $\gamma$ is called the discount factor of the MDP [29, p. 125].

\subsection{MDPIPs}

Additional realism and flexibility can be attached to MDPs by allowing imprecision and indeterminacy in the assessment of transition probabilities. A decision process with states, actions, stages and rewards as described before, but where a set of probability distributions is associated with each transition, has been called a Markov Decision Process with Imprecise Probabilities (MDPIP) by White III and Eldeib [44], a name we adopt in this paper. Satia and Lave Jr. use instead the name MDP with Uncertain Transition Probabilities [31], in what may be the first thorough analysis of this model in the literature; Harmanec uses the term generalized MDP to refer to MDPIPs [21].

MDPIPs can represent incomplete and ambiguous beliefs about transitions between states; conflicting assessments by a group of experts; and situations where one wishes to investigate the effect of perturbations in a "base" model. MDPIPs have also been investigated as representations for abstracted processes, where details about transition probabilities are replaced by an enveloping set of distributions [17, 20]. Similar models are encoded by the controlled Markov set-chains by Kurano et al [26, 24]. Slightly less related are the vector-valued MDPs by Wakuta [41]. Some of these efforts have also adopted interval-valued rewards; in this paper we focus on imprecision/indeterminacy only in transition probabilities.

Thus an MDPIP is composed of a set of stages $\mathcal{T}$, a set of states $\mathcal{S}$, a set of actions $\mathcal{A}$, a reward function $R_{t}$ and sets of probability distributions, each containing transition probabilities $P_{t}$. We assume $\mathcal{T}$ to be the non-negative integers, $\mathcal{S}$ and $\mathcal{A}$ to be finite, and $\mathcal{A}$ to be constant for all states. We assume $R_{t}$ to be a stationary function $R(s, a)$. We also assume stationarity for the sets $K(r \mid s, a)$ of probability distributions. Note, however, that now we have to distinguish two situations. First, the sets of transition probabilities may be identical across stages, while a history of the process may be associated with different draws within these sets (that is, probabilities are selected from sets that do not depend on $t$, but the selection depends on $t$ ). We might refer to these MDPIPs as set-stationary. Alternatively, it may be that each history $h_{t}$ is associated with stationary probability distributions $P\left(s_{t} \mid s_{t-1}, a_{t-1}\right)$ that themselves satisfy the Markov condition (and of course $\left.P\left(s_{t} \mid s_{t-1}, a_{t-1}\right) \in K\left(s_{t} \mid s_{t-1}, a_{t-1}\right)\right)$. We might refer to the second MDPIPs as elementwise-stationary or simply stationary. In this paper we only deal with elementwise-stationary MDPIPs; in fact it does not seem that set-stationary MDPIPs have received any attention in the literature. 
In the remainder of this paper we will use the following notation and terminology regarding sets of probability distributions. A set of probability distributions is called a credal set [27]. The credal set $K(X)$ contains distributions for variable $X$, and the conditional credal set $K(X \mid A)$ contains conditional distributions for variable $X$ given event $A$. Conditioning is elementwise: $K(X \mid A)$ is obtained from $K(X)$ by conditioning every distribution in $K(X)$ on the event $A$. The notation $K(X \mid Y)$ represents a set of credal sets: there is a credal set $K(X \mid Y=y)$ for each nonempty event $\{Y=y\}$. A set of credal sets $K(X \mid Y)$ is separately specified if the joint credal set $K(X, Y)$ is such that, whenever $P\left(X \mid Y=y_{1}\right) \in K\left(X \mid Y=y_{1}\right)$, $P\left(X \mid Y=y_{2}\right) \in K\left(X \mid Y=y_{2}\right)$, then $P\left(X \mid Y=y_{1}\right)$ and $P\left(X \mid Y=y_{2}\right)$ are conditional distributions obtained from a single $P(X, Y)$ in $K(X, Y)$. That is, $K(X \mid Y)$ is separately specified if we can select conditional distributions independently from its sets, an assumption we make throughout for our credal sets. We loosely use $K(r \mid s, a)$ to indicate a separately specified collection of credal sets, for a given action $a$, where $r$ and $s$ refer to states.

Given a credal set $K(X)$, we can compute lower and upper probabilities respectively as $\underline{P}(A)=$ $\inf _{P \in K} P(A)$ and $\bar{P}(A)=\sup _{P \in K} P(A)$. We can also compute lower and upper expectations for any bounded function $f(X)$ as $\underline{E}[f]=\inf _{P \in K} E[f]$ and $\bar{E}[f]=\sup _{P \in K} E[f]$, and likewise for conditional lower/upper probabilities/expectations. We assume all credal sets to be closed, so infima and suprema can be replaced by minima and maxima.

\subsection{Evaluation criteria and algorithms}

Given an MDP that starts at state $s$, we might evaluate a policy $\pi$ by its expected reward:

$$
V_{\pi}(s)=E_{s, \pi}\left[E_{T}\left[\sum_{t=1}^{T} R\left(s_{t}, a_{t}\right)\right]\right] ;
$$

that is, the expectation of the expected reward assuming the process stops at stage $T$. Now if the process has a geometric probability of stopping at $T$, with parameter $\gamma$, we have [29, p. 126]:

$$
V_{\pi, \gamma}(s)=E_{s, \pi}\left[\sum_{t=1}^{\infty} \gamma^{t-1} R\left(s_{t}, a_{t}\right)\right] .
$$

We refer to $V_{\pi, \gamma}(s)$ as the expected total discounted reward. There are other criteria to evaluate policies in MDPs; for example, the expected total reward $E_{s, \pi}\left[\sum_{t=1}^{\infty} R\left(s_{t}, a_{t}\right)\right]$, and the average reward $\lim _{T \rightarrow \infty}(1 / T) E_{s, \pi}\left[\sum_{t=1}^{T} R\left(s_{t}, a_{t}\right)\right][5,29]$. These criteria may be useful in specific problems but they are usually less realistic than Expression (2) and the associated discounted reward (3). We focus on the latter in this paper.

When we move to MDPIPs, we find that several criteria may be used to evaluate policies, even if we adopt total discounted reward. Three possible criteria are:

- Select the policy that yields the largest value of $\min V_{\pi}(s)$, where the minimum applies to all transition probabilities, subject to the fact that these probabilities must belong to given credal sets [4]. That is, the optimal policy produces the highest expected total discounted reward even when probabilities are most unfavorable. This is the $\Gamma$-maximin total discounted reward, where an optimal policy starting from state $s$ must yield

$$
\max _{\pi} \min _{P} V_{\pi, \gamma}(s)
$$

where we append a subscript $P$ in the minimization operator, to emphasize that it applies with respect to all transition probabilities that are imprecise/indeterminate.

- Select the policy that yields, when starting from state $s$,

$$
\max _{\pi} \max _{P} V_{\pi, \gamma}(s) .
$$

That is, both decisions and probabilities can be selected so as to maximize expected total discounted reward. This criterion is referred to as $\Gamma$-maximax total discounted reward.

- Select any policy (or perhaps select all of those policies) that maximizes $V_{\pi, \gamma}(s)$ for at least one choice of transition probabilities. This is the criterion of E-admissibility [27].

Note that $\Gamma$-maximin and $\Gamma$-maximax create a complete order over policies, while E-admissibility is content to explore the partial order of policies induced by credal sets in any convenient way. To date, most authors have adopted the $\Gamma$-maximin criterion. An exception is Harmanec's algorithm [21] which employs interval dominance (Harmanec presents his algorithm as providing maximal policies, however [14, 38] argue that in fact is adopts interval dominance). Several other criteria can be found in the literature $[14,37,38]$.

In this paper we focus on $\Gamma$-maximin total discounted reward; we refer to it as $\Gamma E T D R$ (for Expected Total Discounted Reward $)^{1}$. The work of Satia and Lave

\footnotetext{
${ }^{1}$ It is not our goal to discuss here the adequacy of the $\Gamma$ maximin criterion; it is investigated in this paper because of its wide application in MDP problems. Other criteria will be investigated by the authors in the future. For discussions on the different criterions see [4, 25, 34, 32, 37, 42].
} 
Jr. has derived several important results for this situation [31]. First, there exists a deterministic stationary policy that is optimal. Second, the optimal policy induces a value function that is the unique solution of

$$
V^{*}(s)=\sup _{a} \inf _{P}\left(R(s, a)+\gamma \sum_{r} P(r \mid s, a) V^{*}(r)\right) \text {. }
$$

We can take maximum and minimum in this equation whenever the set of actions $\mathcal{A}$ is finite and the credal sets $K(r \mid s, a)$ have finitely many vertices. We assume this to be true in the remainder of this paper.

Expression (4) can be compactly written as $V^{*}=$ $\mathbf{V} V^{*}$, by lumping the supremum, infimum, and summation into the operator $\mathbf{V}$. Whenever the transition probabilites are fixed (or are precisely specified) at some value $P$, we indicate it through the operator $\mathbf{V}_{P}$ (where the infimum is either suppressed or unnecessary). In fact, for an MDP with transition probabilities $P$, the optimal policy satisfies $V^{*}=\mathbf{V}_{P} V^{*}$, the Bellman equation.

\subsection{Algorithms for MDPs and MDPIPs}

Consider now algorithms that solve the Bellman equation. There are three "classic" algorithms for generating optimal policies in MDPs: value iteration, policy iteration, and reduction to linear programming [5, 29]. Most of the literature focuses on value or policy iteration. However, there are at least three reasons to pay attention to linear programming solutions to MDPs. First, a linear program produces an exact solution without the need to specify any stopping criteria (as needed for value and policy iteration). This property is useful in practice and particularly important while testing other algorithms. Second, several algorithms based on approximating the value function by lower dimensional functions are based on linear programming $[19,22,33]$. Third, and perhaps more importantly, linear programs seem to offer the only organized way to deal with problems where maximization of expected total discounted reward is subject to additional constraints on expected rewards [1, 29].

The linear programming algorithm for MDPs solves the equation $V^{*}=\mathbf{V}_{P} V^{*}$ for the precisely specified transition probabilities as follows [16]:

$$
\begin{array}{ll}
\min _{V^{*}} & \sum_{s} V^{*}(s) \\
\text { s.t. } & V^{*}(s) \geq R(s, a)+\gamma \sum_{r} P(r \mid s, a) V^{*}(r),
\end{array}
$$

where each pair $(s, a)$ corresponds to a constraint.

Policy and value iteration have known counterparts for $\Gamma E T D R$. Satia and Lave Jr. presented a policy iteration algorithm for $\Gamma E T D R$. The results by Satia and Lave Jr., and by Denardo [15], produce a value iteration algorithm as indicated by White III and Eldeib [44]; the same algorithm was later derived in the special case of Bounded-parameter Markov Decision Processes (BMDPs) [17]. The value iteration algorithm starts with a candidate value function $V_{0}^{\prime}(s)$ and iterates:

$$
V_{i+1}^{\prime}=\mathbf{V} V_{i}^{\prime}
$$

until $\left\|V_{i+1}^{\prime}-V_{i}^{\prime}\right\|$ is sufficiently small. ${ }^{2}$ Convergence of this procedure is based on the fact that the operator $\mathrm{V}$ is a contraction mapping. ${ }^{3}$

\section{A multilinear/integer solution for TETDR}

Expression (5) describes the linear program for solving MDPs with precisely specified probabilities. It does not seem possible to produce a linear programming solution for $\Gamma E T D R$; however, as we show in this section, it is possible to generate solutions using well known programming problems. We do not attempt to produce algorithms that surpass value/policy iteration in execution time; rather, our reasons to pursue a programming solution mirror the reasons why others have investigated linear programming for MDPs (summarized in Section 2.4). First, the results produced by multilinear and integer programming, and in particular the latter, depend on combinatorial properties of credal sets, and can be produced exactly; this is useful, for instance, while evaluating other algorithms that only promise $\epsilon$-optimal policies. Second, several approximate algorithms for MDPs that can possibly be extended to MDPIPs depend on linear programming; we conjecture that these potential extensions to MDPIPs will depend on the results in this section. In fact, it seems that multilinear programming is unavoidable in factored models, as we discuss in Section 4. Third, solutions based on optimization seem to be the only way to handle constraints on expected rewards, a topic we wish to pursue in connection with planning (Section 5).

Our main result is, in essence, simple. We start from Expression (4), and note that its solution can be found by solving the following optimization problem:

$$
\begin{array}{ll}
\min _{V^{*}} & \sum_{s} V^{*}(s) \\
\text { s.t. } & V^{*}(s) \geq R(s, a)+\gamma \min _{P} \sum_{r} P(r \mid s, a) V^{*}(r) .
\end{array}
$$

${ }^{2}$ The norm $\|V\|=\max _{s} V(s)$ is typically used in the literature

${ }^{3} \mathrm{~A}$ mapping $\mathbf{V}: U \rightarrow U$, where $U$ is a complete normed linear space, is a contraction mapping iff $\left\|\mathbf{V} u_{1}-\mathbf{V} u_{2}\right\| \leq$ $\gamma\left\|u_{1}-u_{2}\right\|$ for some $\gamma \in[0,1)$. 
This can be shown to be an instance of bilevel programming $[8,40]$. Similar problems have been tackled before in connection with linear programming with uncertainty, with obvious application to $\Gamma E T D R$ $[2,3]$. Current algorithms for bilevel programming are complex, and convergence guarantees are not as sharp as one would like. It would be interesting to reduce Program (7) to a form that were closer to existing, well studied optimization problems. We do this by reducing Program (7) to multilinear and then to integer programming.

The multilinear program we consider is:

$$
\begin{array}{ll}
\min _{V^{*}, P} & \sum_{s} V^{*}(s) \\
\text { s.t. } & V^{*}(s) \geq R(s, a)+\gamma \sum_{r} P(r \mid s, a) V^{*}(r) .
\end{array}
$$

Denote by $\left(V_{R}^{*}, P_{R}^{*}\right)$ a solution of Program (7) and by $\left(V_{G}^{*}, P_{G}^{*}\right)$ a solution of Program (8). In order to use Program (8), we must prove that $V_{G}^{*}$ and $V_{R}^{*}$ are identical.

\section{Theorem $1 \quad V_{G}^{*}=V_{R}^{*}$}

Proof. Let $\Omega_{R}$ and $\Omega_{G}$ be the solution spaces for Programs (7) and (8) respectively. We prove that $\Omega_{R}$ is a subset of $\Omega_{G}$. Then, we show that no solution in $\Omega_{G} \backslash \Omega_{R}$ can have better performance than one in $\Omega_{R}$. We have:

$$
\begin{gathered}
\Omega_{R}=\left\{(V, P): V \in \mathcal{V}, P=\arg \min _{P \in \mathcal{P}} \sum_{r} P(r \mid s, a) V(r)\right\}, \\
\Omega_{G}=\{(V, P): V \in \mathcal{V}, P \in \mathcal{P}\} .
\end{gathered}
$$

Given that the solution space in the second case is the whole space $\mathcal{V} \times \mathcal{P}$, while in the first case $P$ can only be in a subspace $\mathcal{V} \times \mathcal{P}_{R}$ of $\mathcal{V} \times \mathcal{P}$ (hence restricted), Program (8) produces a value function at least as low as Program (7). So, $V_{G}^{*} \leq V_{R}^{*}$, because $\Omega_{G} \supset \Omega_{R}$. Now suppose $V_{G}^{*}<V_{R}^{*}$. For a state $s \in \mathcal{S}$ we have $V_{G}^{*}(s)=R(s, a)+\gamma \sum_{r} P_{G}^{*}(r \mid s, a) V_{G}^{*}(r)$, with $P_{G}^{*}(r \mid s, a) \neq \arg \min _{P} \sum_{r} P(r \mid s, a) V(r)$. If we take $P^{\prime}(r \mid s, a)=\arg \min _{P} \sum_{r} P(r \mid s, a) V(r)$, then $V^{\prime}(s)=$ $R(s, a)+\gamma \sum_{r} P^{\prime}(r \mid s, a) V_{G}^{*}(r)<V_{G}^{*}(s)$ and $V_{G}^{*}$ is not optimal. Since $V_{G}^{*}$ is optimal (given that it considers the whole state space), then $V_{G}^{*} \nless V_{R}^{*}$. This implies that $V_{G}^{*}=V_{R}^{*}$.

Apparently we have moved from a difficult problem (bilevel programming) to another difficult problem (multilinear programming). However, the significance of this result is that multilinear programming is a widely studied field, with close connections to geometric and linear programming [18, 23, 28, 35, 39]. Implementations can deal with hundreds of variables; in our tests we resort to Sherali and Adams' algorithm [35], a branch-and-bound scheme based on linear programming relaxations. Our implementation is an optimized version of this algorithm, that has been used to solve a variety of large and challenging multilinear programs $[10,11,13,12]$. The examples presented later in this section were solved using this implementation.

An even more interesting result obtains if we assume that the vertices of credal sets $K(r \mid s, a)$ are known. Consider a list of vertices (each vertex is a distribution over $\mathcal{S}$ ) for a credal set $K(r \mid s, a),\left\{p_{1}, \ldots, p_{M}\right\}$. Every distribution in this credal set can be expressed as a convex combination $\sum_{i=1}^{M} \alpha_{i} p_{i}$ where $\alpha_{i} \geq 0$ and $\sum_{i} \alpha_{i}=1$. We can then write our goal as:

$$
\begin{aligned}
\min _{V^{*}, \alpha_{i, s, a}} & \sum_{s} V^{*}(s) \\
\text { s.t. } & V^{*}(s) \geq R(s, a)+ \\
& \gamma \sum_{r} \sum_{i} \alpha_{i, s, a} p_{i}(r \mid s, a) V^{*}(r), \\
& \alpha_{i, s, a} \geq 0, \quad \sum_{i} \alpha_{i, s, a}=1,
\end{aligned}
$$

where we explicitly indicate that $\alpha_{i, s, a}$ depends on $(s, a)$.

We now use the fact that a multilinear program has a maximum at the vertices of the credal sets; thus we necessarily have $\alpha_{i, s, a} \in\{0,1\}$ at a solution. We then resort to the following transformation to produce an integer program out of the multilinear program (9), just assuming that we can bound $V^{*}$ from above and below (such bounds can be produced quite generally using results by White III and Eldeib [44]). First, we replace $V^{*}(r) \in[l, u]$ by $l+\left(V^{*}(r)-l\right)$, and create a new variable $\beta_{r}=$ $V^{*}(r)-l \in[0, u-l]$. Each $\alpha_{i, s, a} p_{i}(r \mid s, a) V^{*}(r)$ is thus replaced by $\alpha_{i, s, a} p_{i}(r \mid s, a) l+\alpha_{i, s, a} p_{i}(r \mid s, a) \beta_{r}$. Note that $\alpha_{i, s, a} p_{i}(r \mid s, a) l$ is easy to evaluate. As $\alpha_{i, s, a}$ can be restricted to 0 or 1 , we take each term $\alpha_{i, s, a} p_{i}(r \mid s, a) \beta_{r}$ and replace $\alpha_{i, s, a} \beta_{r}$ by a new variable $\beta_{i, r, s, a}$. To ensure that this replacement does not change the original problem, we introduce linear restrictions:

$$
\begin{gathered}
0 \leq \beta_{i, r, s, a} \leq \beta_{r}, \\
\beta_{i, r, s, a} \leq \alpha_{i, s, a}(u-l), \\
\beta_{r}-(u-l)+\alpha_{i, s, a}(u-l) \leq \beta_{i, r, s, a} .
\end{gathered}
$$

The first and second restrictions are obvious (limitations on $\beta_{r}$ and $\alpha_{i, s, a}$. The last restriction imposes the following. When $\alpha_{i, s, a}=1, \beta_{r} \leq \beta_{i, s, a}$. However, since from the first restriction $\beta_{i, s, a} \leq \beta_{r}$, then $\beta_{i, s, a}=\beta_{r}$, and the full $V^{*}(r)$ will be considered. If $\alpha_{i, s, a}=0$, then $\beta_{r}-(u-l) \leq \beta_{i, r, s, a}$, but 
$\beta_{r}-(u-l)<0$ (since $\left.\beta_{r} \leq(u-l)\right)$, so $\beta_{i, r, s, a}=0$, and this non-optimal pair state-action will not be considered.

We end up with the following integer program:

$$
\begin{aligned}
\min _{V^{*}, \alpha_{i, s, a}} & \sum_{s} V^{*}(s) \\
\text { s.t. } & V^{*}(s) \geq R(s, a)+ \\
& \gamma \sum_{r} \sum_{i}\left[\alpha_{i, s, a} p_{i}(r \mid s, a) l+\right. \\
& \left.p_{i}(r \mid s, a) \beta_{i, r, s, a}\right] \\
& \alpha_{i, s, a} \geq 0, \quad \sum_{i} \alpha_{i, s, a}=1 \\
& \beta_{r}=V^{*}(r)-l \\
& 0 \leq \beta_{r} \leq u-l \\
& 0 \leq \beta_{i, r, s, a} \leq \beta_{r} \\
& \beta_{i, r, s, a} \leq \alpha_{i, s, a}(u-l) \\
& \beta_{r}-(u-l)+\alpha_{i, s, a}(u-l) \leq \beta_{i, r, s, a}
\end{aligned}
$$

We close this section with two examples of MDPIPs. We focus on multilinear programming solutions; later we will consider examples where integer programming is used.

\subsection{A small MDPIP}

This is a very simple, abstract example. Consider two states, $s_{1}$ and $s_{2}$. In each state, the decision maker can choose between two actions. In $s_{1}$ the transition probability for both actions are imprecisely specified, while transition probabilities in $s_{2}$ are precisely specified. Probabilities and rewards are presented in Table 1 (left). The transition probabilities are defined from the states in the first column (origin states) to the states on the first row under $P$ (destination states). The solution given by multilinear programming leads to the optimal solution; the value function $V^{*}$ is shown in Table 1 (right).

\subsection{Planning airplane maintenance through MDPIPs}

This example is based on a problem described by White [43, p. 171]:

An airline classifies the condition of its planes into three categories, viz. excellent, good and poor. The annual running costs for each category are $0.25 \times 10^{6}, 10^{6}$ and $2 \times 10^{6}$ [monetary units] respectively. At the beginning of each year the airline has to decide whether or not to overhaul each plane individually. With no overhaul a plane in excellent condition has probabilities of 0.75 and 0.25 of its condition being excellent or good, respectively, at the beginning of the next year. A plane in good condition has probabilities of 0.67 and 0.33 of its condition being good or poor, respectively, at the beginning of the next year. A plane in poor condition will remain in a poor condition at the beginning of the next year. An overhaul costs $2 \times 10^{6}$ and takes no significant time to do. It restores a plane in any condition to an excellent condition with probability 0.75 , and leaves it in its current condition with probability 0.25 . The airline also has an option of scrapping a plane and replacing it with a new one at a cost of $5 \times 10^{6}$. Such a new plane will be in excellent condition initially. There is an annual discount factor of $\gamma=0.5$.

We consider a variant of this problem where probabilities are specified as in Table 2 (left). Multilinear programming produces the value function in Table 2 (right).

\section{Factored MDPs}

The specification of transitions between states is particularly burdensome in large MDPs. One strategy that has been often employed is to encode transition probabilities in factored form; usually this means that transition probabilities are encoded by Bayesian networks [7]. Here the state space is defined by the configurations of variables $\left\{X_{1}, \ldots, X_{n}\right\}$. We denote by $X_{i, t}$ the $i$ th variable at stage $t$. For each action $a$, we specify a bipartite directed acyclic graph containing $2 n$ nodes denoted by $X_{i}^{+}$and $X_{i}^{-}$; node $X_{i}^{-}$ and $X_{i}^{+}$represent respectively $X_{i, t-1}$ and $X_{i, t}$ for any $t>0$. One layer of the graph contains nodes $X_{i}^{-}$for all $i$, and no edge between them. The other layer contains nodes $X_{i}^{+}$for all $i$, and edges between them. Edges are allowed from nodes in the first layer into the second layer, and also between nodes in the second layer. We denote by $\mathrm{pa}\left(X_{i}^{+}\right)$the parents of $X_{i}^{+}$ in the graph. The graph is assumed endowed with the following Markov condition: a variable $X_{i}^{+}$is conditionally independent of its nondescendants given its parents. This implies the following factorization of transition probabilities:

$$
P\left(X_{1}^{+}, \ldots, X_{n}^{+}\right)=\prod_{i=1}^{n} P\left(X_{i}^{+} \mid \operatorname{pa}\left(X_{i}^{+}\right)\right) .
$$

Now suppose that conditional probability distributions $P\left(X_{i}^{+} \mid \mathrm{pa}\left(X_{i}^{+}\right)\right)$, or a subset of them, are not known precisely, but rather up to inclusion in credal sets $K\left(X_{i}^{+} \mid \mathrm{pa}\left(X_{i}^{+}\right)\right)$. We assume the Markov condition to operate over all combinations of distributions from these credal sets, thus producing a possibly large set of joint distributions, each one of them satisfying 


\begin{tabular}{c|c|c|c|c}
$\mathcal{S}$ & $\mathcal{A}$ & \multicolumn{2}{|c|}{$P$} & \multirow{2}{*}{$R(s, a)$} \\
\cline { 3 - 4 } & & $s_{1}$ & $s_{2}$ & \\
\hline \multirow{2}{*}{$s_{1}$} & $a_{1,1}$ & {$[0,0.5]$} & {$[0.5,1]$} & 7 \\
& $a_{1,2}$ & {$[0,0.2]$} & {$[0.8,1]$} & 3 \\
\hline$s_{2}$ & $a_{2,1}$ & 0.3 & 0.7 & -1 \\
& $a_{2,2}$ & 0.6 & 0.4 & 9
\end{tabular}

\begin{tabular}{|c|c|}
\hline$V^{*}\left(s_{1}\right)$ & 21.486474 \\
\hline$V^{*}\left(s_{2}\right)$ & 18.108099 \\
\hline$\sum_{s} V^{*}(s)$ & 39.594573 \\
\hline
\end{tabular}

Table 1: Specification of simple MDPIP example (left), and value function $V^{*}$ (right).

\begin{tabular}{c|c|c|c|c|c}
\multirow{2}{*}{$\mathcal{S}$} & $\mathcal{A}$ & \multicolumn{3}{|c|}{$P$} & $R(s, a)$ \\
\cline { 3 - 5 } & & $s_{1}$ & $s_{2}$ & $s_{3}$ & \\
\hline \multirow{3}{*}{$s_{1}$} & $a_{1,1}$ & {$[0.5,1]$} & {$[0,0.4]$} & {$[0,0.1]$} & $-0.25 \times 10^{6}$ \\
& $a_{1,2}$ & 1 & 0 & 0 & $-2 \times 10^{6}$ \\
& $a_{1,3}$ & 1 & 0 & 0 & $-5 \times 10^{6}$ \\
\hline \multirow{3}{*}{$s_{2}$} & $a_{2,1}$ & 0 & {$[0.67,1]$} & {$[0,0.33]$} & $-10^{6}$ \\
& $a_{2,2}$ & {$[0.75,1]$} & {$[0,0.25]$} & 0 & $-2 \times 10^{6}$ \\
& $a_{2,3}$ & 1 & 0 & 0 & $-5 \times 10^{6}$ \\
\hline \multirow{3}{*}{$s_{3}$} & $a_{3,1}$ & 0 & 0 & 1 & $-2 \times 10^{6}$ \\
& $a_{3,2}$ & {$[0,0.25]$} & {$[0.5,0.8]$} & {$[0,0.25]$} & $-2 \times 10^{6}$ \\
& $a_{3,3}$ & 1 & 0 & 0 & $-5 \times 10^{6}$
\end{tabular}

\begin{tabular}{|c|c|}
\hline$V^{*}\left(s_{1}\right)$ & -1265664.1604 \\
\hline$V^{*}\left(s_{2}\right)$ & -2496240.6015 \\
\hline$V^{*}\left(s_{3}\right)$ & -4000000.0 \\
\hline$\sum_{s} V^{*}(s)$ & -7761904.7619 \\
\hline
\end{tabular}

Table 2: Specification of MDPIP for plane maintenance (left), and value function $V^{*}$ (right).

the factorization in Expression (11) - the resulting structure is a credal network for each action [9].

The main point of this section is to indicate that Expression (11) defines a multilinear product for the probabilities that appear in Program (8). Thus, the multilinear character of Program (8) is left unchanged: the computation of $\Gamma$-maximin policies is still a matter of multilinear programming. The development of algorithms that produce optimal policies and that exploit the factorization in Expression (11) is left for the future; this is a promising avenue of research as the most advanced algorithms for factored MDPs do use all available structure encoded in the factorization $[19,22]$.

\section{$5 \quad$ MDPSTs}

In this section we explore the properties of a class of MDPIPs that have an important application in the field of artificial intelligence planning. Roughly speaking, planning in artificial intelligence focuses on sequential decision making problems that are specified using high-level languages. There are many variants of AI planning, depending on the properties of the specification language; for example, we have $d e-$ terministic planning, where actions have deterministic effects; probabilistic planning, where actions have probabilistic effects; and nondeterministic planning, where an action may cause a transition to a set of states without any clue at to what state will be moved into [30]. The latter name is somewhat unfortunate as "nondeterminism" is an overloaded term, but it is the usual terminology in the field. Typically deterministic and nondeterministic planning are tackled by search through state spaces, while probabilistic planning is tackled by generation of equivalent MDPs.

There has been considerable effort in the field of AI planning to develop general algorithms that can be instantiated for different types of planning problems [6]. However, until recently no model considered actions with simultaneously "probabilistic" and "nondeterministic" effects. In response to this situation, Trevizan et al. have proposed a jointly probabilistic/nondeterministic framework, based on MDPIPs [36]. Their proposal is based on a class of MDPIPs, called Markov Decision Processes with Setvalued Transitions (MDPSTs), defined as follows.

An MDPST is composed by a set of stages $\mathcal{T}$, a set of states $\mathcal{S}$, a set of actions $\mathcal{A}$, a reward function $R$, a state transition function $F(s, a)$ mapping states $s$ and actions $a \in \mathcal{A}$ into reachable sets of $\mathcal{S}$, i.e., into nonempty subsets of $\mathcal{S}$, and a set of mass assignments $m(k \mid s, a)$ for all $s, a \in \mathcal{A}$, and $k \in F(s, a)$. Here we also assume $\mathcal{T}$ to be the non-negative integers, $\mathcal{S}$ and $\mathcal{A}$ to be finite, $\mathcal{A}$ to be constant for all states, and $R(s, a)$ to be a stationary function. The state transition function $F(s, a)$ and mass assignments $m(k \mid s, a)$ are also stationary. MDPSTs satisfy a simplified ver- 
sion of Expression (4) [36]:

$V^{*}(s)=\max _{a \in \mathcal{A}}\left(R(s, a)+\gamma \sum_{k \in F(s, a)} m(k \mid s, a) \min _{r \in k} V^{*}(r)\right)$.

MDPSTs form a strict subset of MDPIPs [36]; thus Programs (8) or (10) can be used to solve MDPSTs. These solutions require an enumeration on mass assignments $m(k \mid s, a)$. However we can produce simpler programs if we study Expression (12) carefully.

Given any action $a \in \mathcal{A}$, we can collect all feasible $k \in F(s, a)$, and define a binary vector $I(s, a)$ with as many elements as sets of states in $F(s, a)$, such that $I_{i}(s, a) \in\{0,1\}$ for $i \in\{1, \ldots, N\}$, and $\sum_{i} I_{i}(s, a)=1$. Because each $I_{i}(s, a)$ can only be equal to 0 or 1 , and their sum is equal to one, only an unique $I_{i}(s, a)$ can be equal to one at a time. We now write Expression (12) as:

$$
\begin{aligned}
V^{*}(s)= & \max _{a \in \mathcal{A}} R(s, a)+ \\
& \gamma \sum_{k \in F(s, a)} m(k \mid s, a) \sum_{i=1}^{k} I_{i}(s, a) V^{*}\left(r_{i}\right) .
\end{aligned}
$$

We now transform each product $I_{i}(s, a) V^{*}\left(r_{i}\right)$ into a new variable, following the procedure outlined in Section 3. We first replace $V^{*}\left(r_{i}\right)$ by $l+\left(V^{*}\left(r_{i}\right)-l\right)$, where $V^{*}\left(r_{i}\right) \in[l, u]$; we then define $\beta_{i}=V^{*}\left(r_{i}\right)-l$, with $\beta_{i} \in[0, u-l]$. We define a variable $\beta_{i, s, a}=$ $I_{i}(s, a) \beta_{i}$, and add the necessary constraints to the optimization problem. The final integer program is very similar to the Program (10):

$$
\begin{array}{ll}
\min _{V^{*}, I} & \sum_{s} V^{*}(s) \\
\text { s.t. } & V^{*}(s) \geq R(s, a)+ \\
& \gamma \sum_{k} \sum_{i}\left[I_{i}(s, a) m(k \mid s, a) l+\right. \\
& \left.m(k \mid s, a) \beta_{i, s, a}\right] \\
& I_{i}(s, a) \geq 0, \sum_{i} I_{i}(s, a)= \\
& \beta_{i}=V^{*}\left(r_{i}\right)-l \\
& 0 \leq \beta_{i} \leq u-l \\
& 0 \leq \beta_{i, s, a} \leq \beta_{i} \\
& \beta_{i, s, a} \leq I_{i}(s, a)(u-l) \\
& \beta_{i}-(u-l)+I_{i}(s, a)(u-l) \leq \beta_{i, s, a} .
\end{array}
$$

This is a very useful transformation, once integer programming is much simpler than multilevel programming. There are many powerful integer program solvers that guarantee global optimal solutions, where multilevel program solvers only achieve global optimals in certain specific cases.

\subsection{A small MDPST}

Consider 3 states, $s_{1}, s_{2}$ and $s_{3}$. At state $s_{i}$, there are actions $a_{i, 1}$ and $a_{i, 2}$. All actions define probabilistic transitions from one state to itself or to the set composed by the other 2 states, however with different assignments of rewards and transition probabilities. The values assigned to each state and action can be found in Table 3. The optimal solution was obtained by solving an integer program.

\subsection{Probabilistic/nondeterministic planning of airplane maintenance}

Consider the example of airplane maintenance in Section 3. Suppose that transition probabilities follow Table 4 (left); a transition that "fills" more than a column is a nondeterministic one. The optimal solution obtained can be seen in Table 4 (right).

\section{Conclusion}

We have reviewed the basic theory of MDPIPs under the criterion of $\Gamma$-maximin expected total discounted reward, and we have shown how to produce policies using multilinear and integer programming. This type of solution may be useful to handle problems with further constraints on expected rewards, and to deal with factored models and factored approximations. We plan to continue the present work by exactly addressing such constraints and factorizations.

We have then looked into the recently proposed MDPSTs. We have briefly reviewed the application of these processes as a unifying language for "probabilistic" and "nondeterministic" planning, and then showed how these processes nicely lead to integer programming solutions. As indicated previously, one of the reasons to investigate a programming solution for MDPIPs is the promise it holds for treating problems with constraints on policy. For instance, it may be required that a policy, besides maximizing minimum expected total discounted reward, also guarantees the probability of some set of states to be higher than some value (in practice: maximization of profit for a company, subject to the probability that a client is left unattended being smaller than a given value). Markov decision processes subject to such constraints are called constrained MDPs [1, 29], and the main method of solution there is linear programming. We conjecture that constrained MDPIPs will require solutions based on multilinear/integer programming. This will be even more important in the context of MDPSTs, because "nondeterministic" planning is usually associated with contraints on policies. 


\begin{tabular}{c|c|c|c|c}
$\mathcal{S}$ & $\mathcal{A}$ & \multicolumn{2}{|c|}{$P$} & $R(s, a)$ \\
\cline { 3 - 4 } & & $s_{i}$ & $\mathcal{S} \backslash\left\{s_{i}\right\}$ & \\
\hline \multirow{2}{*}{$s_{1}$} & $a_{1,1}$ & 0.8 & 0.2 & 5 \\
& $a_{1,2}$ & 0.1 & 0.9 & -1 \\
\hline$s_{2}$ & $a_{2,1}$ & 0.8 & 0.2 & 4 \\
& $a_{2,2}$ & 0.3 & 0.7 & 7 \\
\hline \multirow{2}{*}{$s_{3}$} & $a_{3,1}$ & 0.7 & 0.3 & 3 \\
& $a_{3,2}$ & 0.25 & 0.75 & 9
\end{tabular}

\begin{tabular}{|c|c|}
\hline$V^{*}\left(s_{1}\right)$ & 17.670251 \\
\hline$V^{*}\left(s_{2}\right)$ & 19.820789 \\
\hline$V^{*}\left(s_{3}\right)$ & 22.153796 \\
\hline$\sum_{s} V^{*}(s)$ & 59.644836 \\
\hline
\end{tabular}

Table 3: Specification of small MDPST (left), and value function $V^{*}$ (right).

\begin{tabular}{|c|c|c|c|c|c|}
\hline \multirow[t]{2}{*}{$\mathcal{S}$} & \multirow[t]{2}{*}{$\mathcal{A}$} & \multicolumn{3}{|c|}{$P$} & \multirow[t]{2}{*}{$R(s, a)$} \\
\hline & & $s_{1}$ & $s_{2}$ & $s_{3}$ & \\
\hline \multirow{3}{*}{$s_{1}$} & $a_{1,1}$ & 0.5 & \multicolumn{2}{|c|}{0.5} & $-0.25 \times 10^{6}$ \\
\hline & $a_{1,2}$ & 1 & 0 & 0 & $-2 \times 10^{6}$ \\
\hline & $a_{1,3}$ & 1 & 0 & 0 & $-5 \times 10^{6}$ \\
\hline \multirow{3}{*}{$s_{2}$} & $a_{2,1}$ & 0 & \multicolumn{2}{|c|}{1} & $-10^{6}$ \\
\hline & $a_{2,2}$ & 0.75 & 0.25 & 0 & $-2 \times 10^{6}$ \\
\hline & $a_{2,3}$ & 1 & 0 & 0 & $-5 \times 10^{6}$ \\
\hline \multirow{3}{*}{$s_{3}$} & $a_{3,1}$ & 0 & 0 & 1 & $-2 \times 10^{6}$ \\
\hline & $a_{3,2}$ & \multicolumn{2}{|c|}{0.8} & 0.2 & $-2 \times 10^{6}$ \\
\hline & $a_{3,3}$ & 1 & 0 & 0 & $-5 \times 10^{6}$ \\
\hline
\end{tabular}

\begin{tabular}{|c|c|}
\hline$V^{*}\left(s_{1}\right)$ & -1666666.6666 \\
\hline$V^{*}\left(s_{2}\right)$ & -3000000.0 \\
\hline$V^{*}\left(s_{3}\right)$ & -4000000.0 \\
\hline$\sum_{s} V^{*}(s)$ & -8666666.666 \\
\hline
\end{tabular}

Table 4: Specification of MDPST for plane maintenance (left), and value function $V^{*}$ (right).

\section{Acknowledgements}

This work has been supported by FAPESP grant 2004/09568-0; the first author is supported by FAPESP grant $05 / 58090-9$; the second author is partially supported by CNPq grant 3000183/98-4; the third author is supported by CAPES grant BEX 424805-8; the fifth author is partially supported by CNPq grant 308530/03-9.

Tests were run in a multilinear programming solver that calls AMPL and CPLEX in its internal loops.

\section{References}

[1] E. Altman. Constrained Markov decision processes. Chapman \& Hall, Boca Raton, Florida, 1999.

[2] I. Averbakh. On the complexity of a class of combinatorial optimization problems with uncertainty. Mathematical Programming, 90(2):263-272, 2001.

[3] A. Ben-Tal and A. Nemirovski. Robust solutions of undertain linear programs. Operations Research Letters, 25(1):1-13, 1993.

[4] J. O. Berger. Statistical Decision Theory and Bayesian Analysis. Springer-Verlag, 1985.

[5] D. P. Bertsekas. Dynamic Programming and Optimal Control (Vol. 1, 2). Athena Scientific, Belmont, Massachusetts, 1995.

[6] B. Bonet and H. Geffner. Learning Depth-First Search: A unified approach to heuristic search in deterministic and non-deterministic settings, and its application to MDPs. In Proc. of the 16th ICAPS, 2006.

[7] C. Boutilier, S. Hanks, and T. Dean. Decisiontheoretic planning: Structural assumptions and computational leverage. Journal of Artificial Intelligence Research, 11:1-94, 1999.

[8] B. Colson, P. Marcotte and G. Savard. Bilevel programming: A survey. Quaterly Journal of Operations Research, 3(2):87-107, 2005.

[9] F. G. Cozman. Graphical models for imprecise probabilities. International Journal of Approximate Reasoning, 39(2-3):167-184, 2005.

[10] F. G. Cozman, C. P. de Campos, J. S. Ide, and J. C. F. da Rocha. Propositional and relational Bayesian networks associated with imprecise and qualitative probabilistic assessments. In Uncertainty in Artificial Intelligence, pages 104-111. AUAI Press, 2004.

[11] C. P. de Campos and F. G. Cozman. Inference in credal networs using multilinear programming. In Second Starting AI Researchers' Symposium (STAIRS), pages 50-61, Amsterdam, IOS Press, 2004.

[12] C. P. de Campos and F. G. Cozman. Belief updating and learning in semi-qualitative probabilistic networks. In Uncertainty in Artificial Intelligence, pages 153-160, Edinburgh, United Kingdom, 2005.

[13] C. P. de Campos and F. G. Cozman. Computing lower and upper expectations under epistemic independence. In Fourth International Symposium on 
Imprecise Probabilities and Their Applications, pages 78-87, Dulles, Virginia, 2005.

[14] G. de Cooman and M. C. M. Troffaes. Dynamic programming for deterministic discrete-time systems with uncertain gain. International Journal Approximate Reasoning, 39(2-3):257-278, 2005.

[15] E. V. Denardo. Contraction mappings in the theory underlying dynamic programming. SIAM Review, 9(2):165-177, 1967.

[16] F. D'Epenoux. A probabilistic production and inventory problem. Management Science, 10(1):98-108, 1963.

[17] R. Givan, S. M. Leach, and T. Dean. Boundedparameter Markov decision processes. Artificial Intelligence, 122(1-2):71-109, 2000.

[18] W. Gochet and Y. Smeers. A branch-and-bound method for reversed geometric programming. Operations Research, 27(5):983-996, September/October 1979.

[19] C. Guestrin, D. Koller, R. Parr, and S. Venkataraman. Efficient solution algorithms for factored MDPs. Journal of Artificial Intelligence Research, 19:399468, 2003.

[20] V. Ha and P. Haddawy. Theoretical foundations for abstraction-based probabilistic planning. In Uncertainty in Artificial Intelligence, pages 291-298, San Francisco, California, United States, 1996. Morgan Kaufmann.

[21] D. Harmanec. Generalizing Markov decision processes to imprecise probabilities. Journal of Statistical Planning and Inference, 105:199-213, 2002.

[22] M. Hauskrecht and B. Kveton. Linear program approximations for factored continuous-state Markov decision processes. In Advances in Neural Information Processing Systems 16, pages 895-902. 2004.

[23] R. Horst and H. Tuy. Global Optimization: Deterministic Approaches. Springer-Verlag, 1995.

[24] M.Hosaka, J. Nakagami, and M. Kurano. Controlled Markov set-chains with set-valued rewards - the average case. International Transactions in Operations Researach, 9:113-123, 2002.

[25] D. Kikuti, F. G. Cozman, and C. P. de Campos. Partially ordered preferences in decision trees: computing strategies with imprecision in probabilities. In $I J$ CAI Workshop on Advances in Preference Handling, pages 118-123, Edinburgh, United Kingdom, 2005.

[26] M. Kurano, J. Song, M. Hosaka, and Y. Huang. Controlled Markov set-chains with discounting. Journal Applied Probability, 35:293-302, 1998.

[27] I. Levi. The Enterprise of Knowledge. MIT Press, Cambridge, Massachusetts, 1980.

[28] C.D. Maranas and C.A. Floudas. Global optimization in generalized geometric programming. Computers and Chemical Engineering, 21(4):351-370, 1997.

[29] M. L. Puterman. Markov Decision Processes. John Wiley and Sons, New York, 1994.
[30] S. J. Russell and P. Norvig. Artificial Intelligence: a Modern Approach. Prentice Hall, New Jersey, 1995.

[31] J. K. Satia and R. E. Lave Jr. Markovian decision processes with uncertain transition probabilities. $O p$ erations Research, 21:728-740, 1970.

[32] M. Schervish, T. Seidenfeld, J. Kadane, and I. Levi. Extensions of expected utility theory and some limitations of pairwise comparisons. In Third International Symposium on Imprecise Probabilities and their Applications, pages 496-510. Carleton Scientific, 2003.

[33] P. J. Schweitzer and A. Seidmann. Generalized polynomial approximations in Markovian decision processes. Journal of Mathematical Analysis and Applications, 110(2):568-582, 1985.

[34] T. Seidenfeld. A contrast between two decision rules for use with (convex) sets of probabilities: $\gamma$-maximin versus $e$-admissibility. Synthese, 140(1-2), 2004.

[35] H.D. Sherali and W.P. Adams. A ReformulationLinearization Technique for Solving Discrete and Continuous Nonconvex Problems. Kluwer Academic Publishers, 1999.

[36] F. W. Trevizan, F. G. Cozman, and L. N. de Barros. Planning under risk and Knightian uncertainty. In 20th IJCAI, pages 2023-2028, 2007.

[37] M. C. M. Troffaes. Decision making with imprecise probabilities: A short review. SIPTA Newsletter, pages $4-7,2004$.

[38] M. C. M. Troffaes. Learning and optimal control of imprecise Markov decision processes by dynamic programming using the imprecise Dirichlet model. pages 141-148, Berlin, 2004. Springer.

[39] H. Tuy. Convex Analysis and Global Optimization, volume 22 of Nonconvex Optimization and Its Applications. Kluwer Academic Publishers, 1998.

[40] L. N. Vicente and P. H. Calamai. Bilevel and multilevel programming: A bibliography review. Journal of Global Optimization, 5(3):291-306, 1994.

[41] K. Wakuta. Vector-valued Markov decision processes and the system of linear inequalities. Stochastic Processes and their Applications, 56:159-169, 1995.

[42] P. Walley. Statistical Reasoning with Imprecise Probabilities. Chapman and Hall, London, 1991.

[43] D. J. White. Markov Decision Processes. John Wiley and Sons, 1993.

[44] C. C. White III and H. K. El-Deib. Markov decision processes with imprecise transition probabilities. $O p$ erations Research, 42(4):739-749, July-August 1994. 


\section{Apêndice II - Artigo: Sequential Decision Processes under Act-State Independence with Arbitrary Choice Functions (Versão completa)}




\title{
Sequential Decision Processes under Act-State Independence with Arbitrary Choice Functions
}

\author{
Matthias C. M. Troffaes, Nathan Huntley, Ricardo Shirota Filho
}

February 14, 2012

\section{Introduction}

Making decisions is a fundamental aspect of daily life, and receives, unsurprisingly, increasing attention by the scientific community. Applications of decision theory abound in many areas, including operations research [7, 27], economics [16], engineering $[1,11]$, artificial intelligence planning and scheduling $[4,8]$ and medicine [12].

The traditional approach to decision making is to model uncertainty via probability, and to model preferences via utility. This leads to the principle of maximising expected utility: an agent is rational if she acts as to maximise expected utility (e.g. see Savage [17]). However, it has been argued that classical (or precise) probability assessments cannot represent the kind of uncertainty usually involved in complex decision making problems. For this reason, more general models for uncertainty and utility have been advocated, such as Dempster-Shafer belief functions [5, 20], imprecise probability [26, 24], gametheoretic probability [21], info-gap theory [2], and many more. In this paper we simply consider arbitrary choice functions. Choice functions originate from social choice theory (see for instance Sen [19]), and provide an elegant and unifying framework to study most (if not all) existing decision theories.

A notable, yet often ignored, problem in any theory of decision making is act-state dependence. Act-state dependence arises when decisions influence the agent's beliefs regarding the occurrence of future states: mathematically, in the classical theory, act-state dependence occurs when the agent's probabilities depend on her action. Although this occurs frequently in practical situations, act-state dependence violates Savage's independence axiom or sure-thing principle (as shown in [14]), and has important consequences to the underlying decision process and how one might solve it. For instance, as pointed out by LaValle and Wapman [14], in the precise case one could argue that backward induction in extensive form only makes sense if you enforce the independence axiom and thus obtain a solution which is consistent with the normal form counterpart. However, one could alternatively accept the fact that normal and extensive form need not to agree, and that normal form solutions might suggest suboptimal choices after uncertainties are resolved and the referred axiom is 
violated, as defended by Hazen [9]. Although, in our opinion, this interesting topic certainly requires further examination and discussion, in this paper we have no ambition for solving it. Therefore, throughout this paper, we always assume act-state independence, and focus on the important theoretical results, with clear practical implications, that we can obtain for this case.

The aim of this paper is thus to investigate how sequential decision processes can be solved, under act-state independence, and using arbitrary choice functions. This builds further on previous work by Huntley and Troffaes [10] on subtree perfectness in decision trees. Subtree perfectness roughly says that the restriction of a normal form solution to a subtree coincides with the normal form solution of that subtree, and is similar to the concept of subgame perfectness introduced by Selten Selten [18] in game theory.

We first identify a reasonable requirement that such solutions ought to satisfy under act-state independence, which we call locality, and which essentially represents a very strong form of subtree perfectness for particular decision problems: locality means that the global sequential problem can be solved by solving a local problem at each stage, for each stage taking only into account rewards incurred locally at that stage, and any events observed from previous stages. An important special case of a sequential decision problem which ought to satisfy locality is a sequence of unrelated (in the sense of rewards and information) decision problems. The latter example is philosophically particularly important, because it means that the intuitively logical answer - solving each decision problem separately - coincides with the answer where one models the sequential problem in full and solves them jointly in the normal form. Locality implies this not only for unrelated decision problems, but also for slightly more general situations where information and rewards at each stage depend on previous stages.

We identify necessary and sufficient conditions on choice functions for locality to be satisfied. As an example, we find necessary and sufficient conditions under which the typical choice functions employed in imprecise probability theory ( $\Gamma$-maximin, maximality, E-admissibility, and interval dominance) satisfy locality. Doing so, marginal extension turns out to play a crucial role.

Before we start our investigation, it is useful to consider a classical Markov decision process with discount rate $0 \leq \gamma<1$, and transition probabilities $p_{s t}^{d}$ and rewards $r_{s t}^{d}$ (real-valued, i.e. utilities) for going from state $s$ to $t$ under decision $d$. For a one-stage Markov decision process starting in state $s_{1}$, the optimal expected utility is

$$
V_{1}\left(s_{1}\right)=\max _{d_{1}} \sum_{s_{2}} p_{s_{1} s_{2}}^{d_{1}} r_{s_{1} s_{2}}^{d_{1}}
$$


For a two-stage process starting in state $s_{1}$, the optimal expected utility is

$$
\begin{aligned}
V_{2}\left(s_{1}\right) & =\max _{d_{1}, d_{2}(\cdot)} \sum_{s_{2} s_{3}} p_{s_{1} s_{2}}^{d_{1}}\left(r_{s_{1} s_{2}}^{d_{1}}+\gamma p_{s_{2} s_{3}}^{d_{2}\left(s_{2}\right)} r_{s_{2} s_{3}}^{d_{2}\left(s_{2}\right)}\right) \\
& =\max _{d_{1}} \sum_{s_{2}} p_{s_{1} s_{2}}^{d_{1}}\left(r_{s_{1} s_{2}}^{d_{1}}+\gamma \max _{d_{2}} \sum_{s_{3}} p_{s_{2} s_{3}}^{d_{2}} r_{s_{2} s_{3}}^{d_{2}}\right) \\
& =\max _{d_{1}} \sum_{s_{2}} p_{s_{1} s_{2}}^{d_{1}}\left(r_{s_{1} s_{2}}^{d_{1}}+\gamma V_{1}\left(s_{2}\right)\right)
\end{aligned}
$$

In general,

$$
V_{0}(s)=0 \quad V_{n+1}(s)=\max _{d} \sum_{t} p_{s t}^{d}\left(r_{s t}^{d}+\gamma V_{n}(t)\right)
$$

This corresponds to the usual value iteration algorithm [22, Sec. 4.4] for finding optimal policies in infinite horizon Markov decision processes, with precisely one policy evaluation step and one policy improvement step at each stage.

Under act-state independence, transition probabilities do not depend on the decisions, and the solution turns out to be extremely simple:

$$
V_{n+1}(s)=\left(\max _{d} \sum_{t} p_{s t} r_{s t}^{d}\right)+\gamma \sum_{t} p_{s t} V_{n}(t)
$$

In other words, at every stage, the optimal decision $d^{*}$ can be obtained by solving a simple one-stage problem, and the sequential decision problem reduces to a sequence of static decision problems. In essence, this property is what we will call locality. Of course, under act-state dependence, locality is clearly no longer a reasonable requirement, since in such case Markov decision processes cannot be solved just locally, and backward induction techniques are required.

As stated before, we will not be concerned with the act-state dependent case, however, we will generalise the above result to the case where

- no probabilities are assumed and rewards do not need to be expressed in terms of utility - instead, our knowledge is expressed via an arbitrary choice function,

- rewards can depend on the full state history,

- state spaces and decision spaces can depend on the stage.

Throughout the paper, we clarify the concepts presented, by solving the following toy problem: an agent sequentially bets on the outcome of a coin toss, and aims to maximise his profit over the full sequence.

The paper is structured as follows. Section 2 introduces basic notions such as choice functions, gambles, and events. Section 3 provides precise notation and definitions for sequential decision processes, and explains how choice functions induce optimal policies. Section 4 defines locality, and presents our main 
result: a simple necessary and sufficient condition on choice functions for locality to be satisfied. Section 5 explores these conditions further in case of four specific choice functions induced by coherent lower previsions: maximality, Eadmissibility, $\Gamma$-maximinity, and interval dominance. We conclude with final remarks and a summary of the main results in Section 6 .

\section{Choice Functions on Gambles}

We first introduce some definitions and notation. Let $\Omega$ be the possibility space: the set of all possible states of the world. In this paper, we shall only consider finite possibility spaces. Note that no probabilities over $\Omega$ are assumed at all. Subsets of $\Omega$ are called events.

Let $\mathcal{R}$ be a set of rewards. Commonly, rewards are measured in utiles, and hence $\mathcal{R}=\mathbb{R}$, but this assumption is not necessary for the results in this paper.

A gamble is a function $X: \Omega \rightarrow \mathcal{R}$; in other words, gambles are $\Omega-\mathcal{R}$ functions. Gambles are interpreted as uncertain rewards: should $\omega \in \Omega$ be the true state of the world, the gamble $X$ will yield the reward $X(\omega)$.

When solving a decision problem, ideally one would like to identify a single decision that the subject considers the best, but there is no reason to suppose that this is always possible. The subject might, however, still be able to identify some decisions that he would never consider choosing, and eliminate these. This leaves a subset of normal form decisions that the subject would be willing to choose from. We say that the subject considers elements of this subset to be optimal.

For instance, in classical decision theory, each decision induces a random realvalued gain, and assuming that all probabilities are fully specified, a decision is considered optimal if its expected gain is maximised. As another example, consider the situation where the probabilities are not precisely known, but a set $\mathcal{M}$ of plausible probability distributions can be specified. Then the subject might consider as optimal any of those decisions whose expected gain is maximal under at least one probability distribution in $\mathcal{M}$. In other situations one might use a different optimality criterion; various criteria will be explored in Section 5 .

In these two examples, optimal decisions are determined by comparison of gambles. This is a common approach, and one we follow here. We therefore suppose that the subject has some way of determining an optimal subset of any set of gambles, conditional upon an event $A$ :

Definition 1. A choice function opt is an operator that, for any non-empty event $A$, maps each non-empty finite set $\mathcal{X}$ of gambles to a non-empty subset of this set:

$$
\emptyset \neq \operatorname{opt}(\mathcal{X} \mid A) \subseteq \mathcal{X}
$$

Note that common uses of choice functions in social choice theory, such as by Sen $[19$, p. 63, ll. 19-21] do not consider conditioning, and define choice functions for arbitrary sets of options (not for gambles only). We follow De Cooman and Troffaes [3] in denoting choice functions by opt. 
Also note that our use of a choice function for solving decision problems enforces act-state independence: if decisions would influence states, then decisions could not be compared by merely considering their gambles.

\section{Solving Sequential Decision Processes Using Arbitrary Choice Functions}

\subsection{Sequential Decision Processes}

As we will be concerned with sequential decision processes, we will consider for $\Omega$ a Cartesian product of state spaces $S_{0}, S_{1}, S_{2}, \ldots, S_{n}$ :

$$
\Omega=S_{0} \times S_{1} \times \cdots \times S_{n}
$$

where $S_{0}$ is the set of possible states of the system at time 0 , and so on. Particular elements of these spaces are denoted by $s_{0}, s_{1}, \ldots, s_{n}$. We identify any such element $s_{k}$ also with an event $E_{s_{k}}=\left\{\left(s_{0}^{\prime}, \ldots, s_{n}^{\prime}\right): s_{k}^{\prime}=s_{k}\right\}$. For brevity, we will sometimes write $s_{k}$ instead of $E_{s_{k}}$ when no confusion is possible, for instance when conditioning.

The states are observed sequentially, and after each observation, we can take a decision from some set, and receive a reward from a set $\mathcal{R}$. With $h_{k}=$ $\left(s_{0}, \ldots, s_{k}\right)$, we can describe the process as:

- observe $s_{0} \in S_{0}$,

- choose $d_{1} \in D_{1}$, observe $s_{1} \in S_{1}$, receive $r_{1}\left(s_{0} d_{1} s_{1}\right)=r_{1}\left(h_{0} d_{1} s_{1}\right)$,

- choose $d_{2} \in D_{2}$, observe $s_{2} \in S_{2}$, receive $r_{2}\left(s_{0} s_{1} d_{2} s_{2}\right)=r_{2}\left(h_{1} d_{2} s_{2}\right)$,

-...

- choose $d_{n} \in D_{n}$, observe $s_{n} \in S_{n}$, receive $r_{n}\left(h_{n-1} d_{n} s_{n}\right)$.

The total reward resulting from the above process is again assumed to be an element of $\mathcal{R}$. More precisely, we assume an operator + on $\mathcal{R}$ which maps every two elements $r$ and $r^{\prime}$ of $\mathcal{R}$ to another element $r+r^{\prime}$ of $\mathcal{R}$. To avoid some technical details in results, we may assume that + has an identity element 0 ; no further properties of + are assumed. To avoid many brackets in our notation, we always let + evaluate from right to left. The total reward is assumed to be:

$$
r_{1}\left(h_{0} d_{1} s_{1}\right)+r_{2}\left(h_{1} d_{2} s_{2}\right)+\cdots+r_{n}\left(h_{n-1} d_{n} s_{n}\right)
$$

Finally, we assume that our preferences over any finite set $\mathcal{X}$ of gambles on $F_{k+1}=S_{k+1} \times \cdots \times S_{n}$ (where $\left.0 \leq k<n\right)$, given any event $h_{k}=s_{0} \ldots s_{k}$, can be represented by a choice function opt $\left(\mathcal{X} \mid h_{k}\right)$.

The process described above is a special case of a sequential decision problem (or, decision tree), in the sense that: 
- chance and decision nodes follow each other consecutively, hence the problem consists of clearly defined stages,

- a variable is observed regardless of the history (for instance, you cannot decide to observe a different variable),

- at each stage, a decision incurs a reward, which may depend on the state history, the current decision, and the next state, but not on anything else,

- the final reward is obtained through combining local rewards.

In our toy example, at each stage, the agent must bet on the outcomehence, there are also two possible decisions: heads or tails - and the agent loses one utile if wrong, but gains one utile if right. So,

$$
\begin{gathered}
S_{1}=S_{2}=\cdots=S_{n}=\{H, T\}, \\
D_{1}=D_{2}=\cdots=D_{n}=\left\{d_{H}, d_{T}\right\},
\end{gathered}
$$

and

$$
r_{k}\left(h_{k-1} d_{k} s_{k}\right)= \begin{cases}1 & \text { if } d_{k} \text { matches } s_{k}, \\ -1 & \text { otherwise }\end{cases}
$$

Note that the initial state $s_{0}$ is not of relevance in this problem. For mathematical convenience, we can simply let $S_{0}$ be a singleton.

A traditional approach is to express the agent's beliefs about the coin by means of probabilities. In the simplest case, heads or tails are equally likely to occur. However, in a more realistic setting one usually cannot make this assumption: there might be insufficient information to affirm the coin's fairness. Therefore, we allow the agent to learn about the possible bias of the coin whilst still performing optimally with respect to his existing knowledge.

Before we go on solving our toy problem in detail, we introduce a few more ideas.

\subsection{Normal Form Solution}

\subsubsection{Policies}

A conceptually straightforward way of solving the sequential decision process that we just described, goes as follows. Consider our decision process after a particular sequence of decisions and states $s_{0} d_{1} s_{1} \ldots d_{k-1} s_{k-1}$ has already occurred. A policy for our sequential decision process consists of a specification of a decision $d_{k} \in D_{k}$ and decision functions $d_{k+1}(\cdot): S_{k} \rightarrow D_{k+1}, \ldots$, and $d_{n}(\cdot): S_{k} \times \cdots \times S_{n-1} \rightarrow D_{n}$ :

- $d_{k} \in D_{k}$,

- $d_{k+1}\left(s_{k}\right) \in D_{k+1}$, 
- $d_{n}\left(s_{k} \ldots s_{n-1}\right) \in D_{n}$,

In the more general context of sequential decision making, a policy is also called a normal form decision. The set of all policies is denoted by $\Pi_{k}^{n}$ :

$$
\Pi_{k}^{n}=D_{k} \times D_{k+1}^{S_{k}} \times \cdots \times D_{n}^{S_{k} \times \cdots \times S_{n-1}}
$$

In our toy example, a policy could be 'always bet tails', or 'always bet heads', or 'bet tails on odd stages, and heads on even stages', or even 'bet tails if we have observed more tails than heads in the past, otherwise bet heads'. Of course there are many other policies, and these are just a few.

\subsubsection{Gambles}

Each state history $s_{0} s_{1} \ldots s_{k-1}=h_{k-1}$ in $H_{k-1}$ and each policy $\pi_{k}^{n}=\left(d_{k}, d_{k+1}(\cdot), \ldots, d_{n}(\cdot)\right)$ in $\Pi_{k}^{n}$ incurs a gamble $X_{k}^{n}\left(h_{k-1}, \pi_{k}^{n}\right)$, that is, a mapping from $S_{k} \times \cdots \times S_{n}$ to $\mathcal{R}:$

$$
\begin{array}{r}
\bigoplus_{s_{k}} E_{s_{k}} \bigoplus_{s_{k+1}} E_{s_{k+1}} \ldots \bigoplus_{s_{n}} E_{s_{n}}\left(r_{k}\left(h_{k-1} d_{k} s_{k}\right)+r_{k+1}\left(h_{k} d_{k+1}\left(h_{k}\right) s_{k+1}\right)+\ldots\right. \\
\left.\cdots+r_{n}\left(h_{n-1} d_{n}\left(h_{n-1}\right) s_{n}\right)\right)
\end{array}
$$

which we can also write as

$$
\begin{array}{r}
\bigoplus_{s_{k}} E_{s_{k}}\left(r_{k}\left(h_{k-1} d_{k} s_{k}\right)+\bigoplus_{s_{k+1}} E_{s_{k+1}}\left(r_{k+1}\left(h_{k} d_{k+1}\left(h_{k}\right) s_{k+1}\right)+\ldots\right.\right. \\
\left.\left.\cdots+\bigoplus_{s_{n}} E_{s_{n}} r_{n}\left(h_{n-1} d_{n}\left(h_{n-1}\right) s_{n}\right)\right) \ldots\right)
\end{array}
$$

For the sake of brevity, we have slightly abused notation: $d_{\ell}\left(h_{\ell-1}\right)$ denotes of course simply $d_{\ell}\left(s_{k} \ldots s_{\ell-1}\right)$.

The gamble $X_{k}^{n}\left(h_{k-1}, \pi_{k}^{n}\right)$ describes the reward $X_{k}^{n}\left(h_{k-1}, \pi_{k}^{n}\right)\left(f_{k}\right)$ that we receive for each possible sequence of future states $f_{k}=\left(s_{k}, \ldots, s_{n}\right)$ when we follow policy $\pi_{k}^{n}$ after having observed state history $h_{k-1}$.

The gamble $X_{k}^{n}\left(h_{k-1}, \pi_{k}^{n}\right)$ generalises the classical value function $V\left(h_{k-1}, \pi_{k}^{n}\right)$ of a policy at a given state history to the case where probabilities are not given and rewards are not assumed to be expressed in utiles. Indeed, if we were given a probability $p\left(\cdot \mid h_{k-1}\right)$ on $F_{k}=S_{k} \times \cdots \times S_{n}$, and rewards were expressed in utiles, then the expected value of a given policy $\pi_{k}^{n}$ at state history $h_{k-1}$ would be precisely

$$
V\left(h_{k-1}, \pi_{k}^{n}\right)=\sum_{f_{k}} p\left(f_{k} \mid h_{k-1}\right) X_{k}^{n}\left(h_{k-1}, \pi_{k}^{n}\right)\left(f_{k}\right)
$$


Again, considering our toy example, the gamble corresponding to the policy 'always bet tails' would be:

$$
X_{k}^{n}\left(h_{k-1}, \pi_{k}^{n}\right)\left(f_{k}\right)=n_{T}-n_{H}
$$

where $n_{T}$ is the number of tails in $f_{k}$, and $n_{H}$ the number of heads in $f_{k}$, because under the given policy 'always bet tails', we gain one utile for each tail in $f_{k}$, and lose one utile for each head in $f_{k}$. As an other example, in a single stage problem, the policy 'bet heads' has gamble:

$$
X_{k}^{k}\left(h_{k-1}, d_{H}\right)\left(s_{k}\right)= \begin{cases}1 & \text { if } s_{k}=H \\ -1 & \text { if } s_{k}=T\end{cases}
$$

\subsubsection{Optimal Policies}

A policy $\pi_{k}^{n}$ is optimal for a given state history $h_{k-1}$ if

$$
X_{k}^{n}\left(h_{k-1}, \pi_{k}^{n}\right) \in \operatorname{opt}\left(\mathcal{X}_{k}^{n}\left(h_{k-1}\right) \mid h_{k-1}\right)
$$

where $\mathcal{X}_{k}^{n}\left(h_{k-1}\right)$ is the set of gambles incurred by all policies when we start from state history $h_{k-1}$. The set of all optimal policies, for a given state history $h_{k-1}$, is denoted by $\Pi_{k}^{n}\left(h_{k-1}\right)$. So,

$$
\begin{aligned}
\Pi_{k}^{n}\left(h_{k-1}\right) & =\underset{\pi_{k}^{n} \in \Pi_{k}^{n}}{\arg \operatorname{opt}}\left(X_{k}^{n}\left(h_{k-1}, \pi_{k}^{n}\right) \mid h_{k-1}\right) \\
& =\left\{\pi_{k}^{n} \in \Pi_{k}^{n}: X_{k}^{n}\left(h_{k-1}, \pi_{k}^{n}\right) \in \operatorname{opt}\left(\mathcal{X}_{k}^{n}\left(h_{k-1}\right) \mid h_{k-1}\right)\right\}
\end{aligned}
$$

The set of optimal gambles opt $\left(\mathcal{X}_{k}^{n}\left(h_{k-1}\right) \mid h_{k-1}\right)$ generalises of course the classical (optimal) value function $V\left(h_{k-1}\right)$ at a given state history to the case where probabilities are not given and rewards are not assumed to be expressed in utiles. Indeed, if we were given a joint probability $p$ on $F_{k}=S_{k} \times \cdots \times S_{n}$, and rewards were expressed in utiles, then the (optimal) value function at $h_{k-1}$ would be

$$
V\left(h_{k-1}\right)=\max _{\pi_{k}^{n} \in \Pi_{k}^{n}} V\left(h_{k-1}, \pi_{k}^{n}\right)
$$

which is, for this case, the expectation of (any element of) $\operatorname{opt}\left(\mathcal{X}_{k}^{n}\left(h_{k-1}\right) \mid h_{k-1}\right)$.

\section{Locality}

As seen previously, under act-state independence, we can solve an $n$-stage Markov decision process simply by solving $n$ one-stage ones. We now generalise this idea.

To express locality conveniently, we first introduce some further notation. Let $\Pi_{k}^{k}(\cdot)$ denote all locally optimal decision functions:

$$
\Pi_{k}^{k}(\cdot)=\left\{d_{k}(\cdot) \in\left(D_{k}\right)^{H_{k-1}}: d_{k}\left(h_{k-1}\right) \in \Pi_{k}^{k}\left(h_{k-1}\right)\right\}
$$


(It may be useful at this point to recall that $\Pi_{k}^{k}=D_{k}$.) More generally,

$$
\begin{aligned}
& \Pi_{k}^{n}(\cdot)=\left\{\left(d_{k}(\cdot), d_{k+1}(\cdot), \ldots, d_{n}(\cdot)\right) \in\left(\Pi_{k}^{n}\right)^{H_{k-1}}:\right. \\
&\left.\left(d_{k}\left(h_{k-1}\right), d_{k+1}\left(h_{k-1} \cdot\right), \ldots, d_{n}\left(h_{k-1} \cdot\right)\right) \in \Pi_{k}^{n}\left(h_{k-1}\right)\right\}
\end{aligned}
$$

where we used the identity

$$
\begin{aligned}
\left(\Pi_{k}^{n}\right)^{H_{k-1}} & =\left(D_{k} \times D_{k+1}^{S_{k}} \times \cdots \times D_{n}^{S_{k} \times \cdots \times S_{n-1}}\right)^{H_{k-1}} \\
& =D_{k}^{H_{k-1}} \times D_{k+1}^{H_{k-1} \times S_{k}} \times \cdots \times D_{n}^{H_{k-1} \times S_{k} \times \cdots \times S_{n-1}} \\
& =D_{k}^{H_{k-1}} \times D_{k+1}^{H_{k}} \times \cdots \times D_{n}^{H_{n-1}}
\end{aligned}
$$

With any opt on $S_{0}, \ldots, S_{n}$, we can associate the following property:

Property 1 (Locality). We say that opt satisfies locality (on $S_{0}, \ldots, S_{n}$ ) whenever, for each sequential decision process on $S_{0}, \ldots, S_{n}$ and each $1 \leq k<$ $n$,

$$
\Pi_{k}^{n}(\cdot)=\Pi_{k}^{k}(\cdot) \times \Pi_{k+1}^{k+1}(\cdot) \times \cdots \times \Pi_{n}^{n}(\cdot) .
$$

Perhaps surprisingly, locality on two stage problems is (necessary and) sufficient for locality on problems of any number of stages:

Lemma 2. For every $1 \leq k<n$,

$$
\Pi_{k}^{n}(\cdot)=\Pi_{k}^{k}(\cdot) \times \Pi_{k+1}^{n}(\cdot)
$$

if and only if, for every $1 \leq k<n$,

$$
\Pi_{k}^{n}(\cdot)=\Pi_{k}^{k}(\cdot) \times \Pi_{k+1}^{k+1}(\cdot) \times \cdots \times \Pi_{n}^{n}(\cdot) .
$$

Proof. Apply the given equality recursively:

$$
\begin{aligned}
\Pi_{k}^{n}(\cdot) & =\Pi_{k}^{k}(\cdot) \times \Pi_{k+1}^{n}(\cdot) \\
& =\Pi_{k}^{k}(\cdot) \times \Pi_{k+1}^{k+1}(\cdot) \times \Pi_{k+2}^{n}(\cdot) \\
& =\ldots \\
& =\Pi_{k}^{k}(\cdot) \times \Pi_{k+1}^{k+1}(\cdot) \times \cdots \times \Pi_{n}^{n}(\cdot)
\end{aligned}
$$

\subsection{Sequential Distributivity}

Recall, $H_{k}=S_{0} \times \cdots \times S_{k}$, and $F_{k}=S_{k} \times \cdots \times S_{n}$. With any opt on $S_{0}, \ldots$, $S_{n}$, we can associate the following property:

Property 2 (Sequential Distributivity). For any $1 \leq k<n$, any value $h_{k-1}$ of $H_{k-1}$, all finite sets of gambles $\mathcal{X}$ on $S_{k}$, all finite sets of gambles $\mathcal{Y}\left(s_{k}\right)$ on $F_{k+1}$ (one such set for each $s_{k} \in S_{k}$ ), and all $X \in \mathcal{X}$ and $Y\left(s_{k}\right) \in \mathcal{Y}\left(s_{k}\right)$ :

$$
\begin{aligned}
X+\bigoplus_{s_{k}} E_{s_{k}} Y\left(s_{k}\right) \in \operatorname{opt}\left(\mathcal{X}+\bigoplus_{s_{k}} E_{s_{k}} \mathcal{Y}\left(s_{k}\right) \mid h_{k-1}\right) \\
\Longleftrightarrow X \in \operatorname{opt}\left(\mathcal{X} \mid h_{k-1}\right) \text { and } Y\left(s_{k}\right) \in \operatorname{opt}\left(\mathcal{Y}\left(s_{k}\right) \mid h_{k-1} s_{k}\right) \text { for all } s_{k} .
\end{aligned}
$$


We shall show that the above property is necessary and sufficient for our normal form solution induced by opt to reduce to a sequence of $n$ single-stage normal form solutions.

Theorem 3. opt satisfies locality if and only if opt satisfies sequential distributivity.

Proof. Before we start the proof, note that we can write $\Pi_{k}^{n}$ as

$$
\begin{aligned}
\Pi_{k}^{n} & =D_{k} \times D_{k+1}^{S_{k}} \times \cdots \times D_{n}^{S_{k} \times \cdots \times S_{n-1}} \\
& =D_{k} \times\left(D_{k+1} \times D_{k+2}^{S_{k+1}} \times \cdots \times D_{n}^{S_{k+1} \times \cdots \times S_{n-1}}\right)^{S_{k}} \\
& =D_{k} \times\left(\Pi_{k+1}^{n}\right)^{S_{k}}
\end{aligned}
$$

In other words, any policy $\pi_{k}^{n}$ can be interpreted as a 2-stage policy, where we first choose $d_{k} \in D_{k}$, and then $\pi_{k+1}^{n}\left(s_{k}\right) \in \Pi_{k+1}^{n}$ depending on the event $s_{k}$ that occurred. Throughout the proof, we will make use of this correspondence, and typically write $\pi_{k}^{n}$ as $\left(d_{k}, \pi_{k+1}^{n}(\cdot)\right)$ instead of the more usual $\left(d_{k}, d_{k+1}(\cdot), \ldots, d_{n}(\cdot)\right)$.

"if". We must show that opt satisfies locality, given that it satisfies sequential distributivity. By Lemma 2, it suffices to show that $\Pi_{k}^{n}(\cdot)=\Pi_{k}^{k}(\cdot) \times \Pi_{k+1}^{n}(\cdot)$ for all $1 \leq k<n$.

Consider any $1 \leq k<n$. For every $h_{k-1}$, we must show that $\pi_{k}^{n *}=$ $\left(d_{k}^{*}, \pi_{k+1}^{n *}(\cdot)\right) \in \Pi_{k}^{n}\left(h_{k-1}\right)$ if and only if $d_{k}^{*} \in \Pi_{k}^{k}\left(h_{k-1}\right)$ and $\pi_{k+1}^{n *}\left(s_{k}\right) \in \Pi_{k+1}^{n}\left(h_{k-1} s_{k}\right)$ for every $s_{k}$. Equivalently, we show that, regardless of $h_{k-1}$,

$$
X_{k}^{n}\left(h_{k-1}, \pi_{k}^{n *}\right) \in \operatorname{opt}\left(\mathcal{X}_{k}^{n}\left(h_{k-1}\right) \mid h_{k-1}\right)
$$

if and only if

$$
\begin{aligned}
X_{k}^{k}\left(h_{k-1}, d_{k}^{*}\right) & \in \operatorname{opt}\left(\mathcal{X}_{k}^{k}\left(h_{k-1}\right) \mid h_{k-1}\right) \text { and } \\
X_{k+1}^{n}\left(h_{k-1} s_{k}, \pi_{k+1}^{n *}\left(s_{k}\right)\right) & \in \operatorname{opt}\left(\mathcal{X}_{k+1}^{n}\left(h_{k-1} s_{k}\right) \mid h_{k-1} s_{k}\right) \text { for all } s_{k} .
\end{aligned}
$$

First, note that, for any $\pi_{k}^{n}=\left(d_{k}, \pi_{k+1}^{n}(\cdot)\right) \in \Pi_{k}^{n}$,

$$
X_{k}^{n}\left(h_{k-1}, \pi_{k}^{n}\right)=X_{k}^{k}\left(h_{k-1}, d_{k}\right)+\bigoplus_{s_{k}} E_{s_{k}} X_{k+1}^{n}\left(h_{k-1} s_{k}, \pi_{k+1}^{n}\left(s_{k}\right)\right)
$$

simply by the definitions of $X_{k}^{n}\left(h_{k-1}, \pi_{k}^{n}\right), X_{k}^{k}\left(h_{k-1}, d_{k}\right)$, and $X_{k+1}^{n}\left(h_{k-1} s_{k}, \pi_{k+1}^{n}\left(s_{k}\right)\right)$. Taking the union over all $\pi_{k}^{n} \in \Pi_{k}^{n}$, it follows that also

$$
\mathcal{X}_{k}^{n}\left(h_{k-1}\right)=\mathcal{X}_{k}^{k}\left(h_{k-1}\right)+\bigoplus_{s_{k}} E_{s_{k}} \mathcal{X}_{k+1}^{n}\left(h_{k-1} s_{k}\right) \text {. }
$$

Now, simply apply sequential distributivity, and use Eqs. (12) and (13) to see that Eq. (9) holds if and only if Eqs. (10) and (11) hold.

"only if". Let $1 \leq k<n$. By the locality assumption, we know that $\Pi_{k}^{n}(\cdot)=\Pi_{k}^{k}(\cdot) \times \Pi_{k+1}^{n}(\cdot)$. We show that sequential distributivity holds. 
Indeed, consider any value $h_{k-1}$ of $H_{k-1}$, any finite set of gambles $\mathcal{X}=$ $\left\{X_{1}, \ldots, X_{p}\right\}$ on $S_{k}$, any finite sets of gambles $\mathcal{Y}\left(s_{k}\right)=\left\{Y_{1}\left(s_{k}\right), \ldots, Y_{q}\left(s_{k}\right)\right\}$ on $F_{k+1}$ (one such set for each $s_{k} \in S_{k}$ ) -for simplicity of notation, for each $s_{k}$ we use an index set for $\mathcal{Y}\left(s_{k}\right)$ of the same size $q$ : this goes without loss of generality as we can always allow some of the $Y_{j}\left(s_{k}\right)$ to be equal to one another - and any $X_{i} \in \mathcal{X}$ and $Y_{j\left(s_{k}\right)}\left(s_{k}\right) \in \mathcal{Y}\left(s_{k}\right)$ - so $j(\cdot)$ is a mapping from $S_{k}$ to $\{1, \ldots, q\}$.

We must show that

$$
\begin{aligned}
& X_{i}+\bigoplus_{s_{k}} E_{s_{k}} Y_{j\left(s_{k}\right)}\left(s_{k}\right) \in \operatorname{opt}\left(\mathcal{X}+\bigoplus_{s_{k}} E_{s_{k}} \mathcal{Y}\left(s_{k}\right) \mid h_{k-1}\right) \\
& \Longleftrightarrow X_{i} \in \operatorname{opt}\left(\mathcal{X} \mid h_{k-1}\right) \text { and } Y_{j\left(s_{k}\right)}\left(s_{k}\right) \in \operatorname{opt}\left(\mathcal{Y}\left(s_{k}\right) \mid h_{k-1} s_{k}\right) \text { for all } s_{k}
\end{aligned}
$$

To this end, consider any $n$-stage process where

$$
\begin{aligned}
D_{k} & =\left\{x_{1}, \ldots, x_{p}\right\} \\
D_{k+1}=\cdots=D_{n-1} & =\{d\} \\
D_{n} & =\left\{y_{1}, \ldots, y_{q}\right\}
\end{aligned}
$$

and

$$
\begin{aligned}
r_{k}\left(h_{k-1} x_{i} s_{k}\right) & =X_{i}\left(s_{k}\right) \\
r_{k+1}\left(h_{k} d s_{k+1}\right)=\cdots=r_{n-1}\left(h_{n-2} d s_{n-1}\right) & =0 \\
r_{n}\left(h_{n-1} y_{j} s_{n}\right) & =Y_{j\left(s_{k}\right)}\left(s_{k}\right)\left(f_{k+1}\right)
\end{aligned}
$$

where 0 is the identity element in $\mathcal{R}$ with respect to + . Observe that, for this process,

$$
\begin{aligned}
\mathcal{X}_{k}^{k}\left(h_{k-1}\right) & =\mathcal{X}, \\
\mathcal{X}_{k+1}^{n}\left(h_{k-1} s_{k}\right) & =\mathcal{Y}\left(s_{k}\right),
\end{aligned}
$$

and, as shown before,

$$
\mathcal{X}_{k}^{n}\left(h_{k-1}\right)=\mathcal{X}+\bigoplus_{s_{k}} E_{s_{k}} \mathcal{Y}\left(s_{k}\right)
$$

But, note that $\Pi_{k}^{n}(\cdot)=\Pi_{k}^{k}(\cdot) \times \Pi_{k+1}^{n}(\cdot)$ means that, for any $\pi_{k}^{n}=\left(x_{i}, y_{j(\cdot)}\right) \in \Pi_{k}^{n}$,

$$
\begin{aligned}
& \left(x_{i}, y_{j(\cdot)}\right) \in \Pi_{k}^{n}\left(h_{k-1}\right) \\
& \qquad x_{i} \in \Pi_{k}^{k}\left(h_{k-1}\right) \text { and } y_{j\left(s_{k}\right)} \in \Pi_{k+1}^{n}\left(h_{k-1} s_{k}\right) \text { for all } s_{k} .
\end{aligned}
$$

By definition of $\Pi_{k}^{n}\left(h_{k-1}\right), \Pi_{k}^{k}\left(h_{k-1}\right)$, and $\Pi_{k+1}^{n}\left(h_{k-1} s_{k}\right)$, and because of what we have just shown about $\mathcal{X}_{k}^{n}\left(h_{k-1}\right), \mathcal{X}_{k}^{k}\left(h_{k-1}\right)$, and $\mathcal{X}_{k+1}^{n}\left(h_{k-1} s_{k}\right)$, this is exactly equivalent to Eq. (14). 


\section{Locality for Choice Functions Induced by Co- herent Lower Previsions}

In this section we derive necessary and sufficient conditions for locality on four types of choice functions induced by coherent lower previsions: maximality, E-admissibility, $\Gamma$-maximin, and interval dominance.

We start with a brief outline of the theory of coherent lower previsions: they generalize the classical theory of probability by bounding probabilities, and are useful when information is scarce or conflicting [24, 15].

First, to work with coherent lower previsions, we must suppose that rewards are expressed in utiles, hence $\mathcal{R}=\mathbb{R}$, and that + simply represents addition. This will be assumed throughout the remainder of this paper, however do note that this is merely a practical assumption in order to work with lower previsions, and our central result, Theorem 3, does not actually depend on rewards being expressed in utiles.

A simple way to introduce coherent lower previsions on a finite possibility space $\Omega$, which also handles conditioning quite naturally, goes via Dubin's full conditional probabilities [6]. Essentially, a full conditional probability $p$ yields a probability $p(A \mid B)$ for every event $A$ and $B(A \subseteq \Omega, \emptyset \neq B \subseteq \Omega)$. Note that we cannot simply start from an unconditional probability, and use Kolmogorov's definition of conditional probability [13], because, as we will see in what follows, zero probability plays an essential role in our results, and Kolmogorov's approach excludes conditioning on events of probability zero.

A full conditional probability $p$ induces a full conditional expectation: for every gamble $X$ on $\Omega$ and every non-empty event $B \subseteq \Omega$, we can define

$$
E_{p}(X \mid B)=\sum_{\omega \in B} p(\omega \mid B) X(\omega)
$$

Using the properties of full conditional probabilities, it is easily proved that $E_{p}$ is a conditional prevision in the sense of Williams $[26, \S 1.2 .1$, p. 10], or a coherent conditional linear prevision in the sense of Walley [24, $\$ 7.1 .4$, p. 346-347].

A coherent conditional lower prevision is then simply the lower envelope of some set $\mathcal{M}$ of coherent conditional previsions $E_{p}$, and the corresponding conditional upper prevision is the upper envelope of $\mathcal{M}$ :

$$
\underline{P}(X \mid B)=\inf _{E_{p} \in \mathcal{M}} E_{p}(X \mid B) \quad \bar{P}(X \mid B)=\sup _{E_{p} \in \mathcal{M}} E_{p}(X \mid B)
$$

Although, for mathematical simplicity, we defined $\underline{P}$ and $\bar{P}$ by means of a set of full conditional probabilities, there are different ways of obtaining and interpreting lower and upper previsions [15]; this is beyond the scope of the paper.

The following properties of coherent conditional lower and upper previsions are well known (see for instance Williams [26]) and will be used further on:

Proposition 4. For every non-empty event $A \subseteq \Omega$, every gamble $X$ and $Y$ on $\Omega$, every real number $\mu$, and every non-negative real number $\lambda$, the following 
statements hold. *** TODO add references in text where these are used, check if more are needed ***

(i) $\underline{P}(X \mid A)=-\bar{P}(-X \mid A)$.

(ii) If $A X=A Y$ then $\underline{P}(X \mid A)=\underline{P}(Y \mid A)$ and $\bar{P}(X \mid A)=\bar{P}(Y \mid A)$.

(iii) $\underline{P}(X \mid A)+\underline{P}(Y \mid A) \leq \underline{P}(X+Y \mid A) \leq \underline{P}(X \mid A)+\bar{P}(Y \mid A) \leq \bar{P}(X+Y \mid A)$.

(iv) $\underline{P}(\lambda X \mid A)=\lambda \underline{P}(X \mid A)$ and $\bar{P}(\lambda X \mid A)=\lambda \bar{P}(X \mid A)$.

(v) If $X(\omega) \geq Y(\omega)$ for all $\omega \in A$, then $\underline{P}(X \mid A) \geq \underline{P}(Y \mid A)$ and $\bar{P}(X \mid A) \geq$ $\bar{P}(Y \mid A)$.

(vi) $\underline{P}(X+\mu \mid A)=\underline{P}(X \mid A)+\mu$ and $\bar{P}(X+\mu \mid A)=\bar{P}(X \mid A)+\mu$.

Observe that, by (i), $\bar{P}$ is completely determined by $\underline{P}$ : therefore, we usually only refer to $\underline{P}$.

The next property of coherent conditional lower previsions will reoccur frequently in our study. Remember that $H_{k}=S_{0} \times \cdots \times S_{k}$, and $F_{k}=S_{k} \times \cdots \times S_{n}$.

Definition 5. Let the possibility space be $\Omega=S_{0} \times \cdots \times S_{n}$. A coherent lower prevision $\underline{P}$ is then said to satisfy marginal extension (with respect to $S_{0}, \ldots$, $S_{n}$ ) whenever, for all $1 \leq k<n$, all gambles $Z$ on $F_{k}$, and all $h_{k-1} \in H_{k-1}$,

$$
\underline{P}\left(Z \mid h_{k-1}\right)=\underline{P}\left(\underline{P}\left(Z \mid h_{k-1} S_{k}\right) \mid h_{k-1}\right)
$$

In the above definition, $\underline{P}\left(Z \mid h_{k-1} S_{k}\right)$ denotes the gamble

$$
\underline{P}\left(Z \mid h_{k-1} S_{k}\right): s_{k} \mapsto \underline{P}\left(Z \mid h_{k-1} s_{k}\right)
$$

Note that the order of the state spaces is relevant for marginal extension. For instance, satisfying marginal extension with respect to $S_{0}, S_{1}, S_{2}$, is not equivalent to satisfying marginal extension with respect to $S_{0}, S_{2}, S_{1}$.

Finally, note that for conditional previsions $E_{p}$, marginal extension corresponds to disintegrability [6, p. 90, Eq. (3)], and hence is always satisfied in our case (since we are concerned with finite state spaces only).

\subsection{Maximality}

Assume that we are given a coherent lower prevision $\underline{P}(\cdot \mid \cdot)$ - by natural (or regular) extension, we may assume without loss of generality that $\underline{P}(\cdot \mid \cdot)$ is defined on all gambles on $S_{0} \times \cdots \times S_{n}$, and conditional on all non-empty events in $S_{0} \times \cdots \times S_{n}$. Then, a policy $\pi_{k}^{n *} \in \Pi_{k}^{n}$ is optimal, in the sense of maximality, whenever

$$
\bar{P}\left(X_{k}^{n}\left(h_{k-1}, \pi_{k}^{n *}\right)-X_{k}^{n}\left(h_{k-1}, \pi_{k}^{n}\right) \mid h_{k-1}\right) \geq 0
$$

for all policies $\pi_{k}^{n} \in \Pi_{k}^{n}$.

Proposition 6. Maximality with respect to a coherent lower prevision satisfies locality on $S_{0}, \ldots, S_{n}$, if and only if 
(i) $\underline{P}\left(E_{s_{k}} \mid h_{k-1}\right)>0$ for all $1 \leq k<n, h_{k-1} \in H_{k-1}$, and $s_{k} \in S_{k}$, and

(ii) $\underline{P}$ satisfies marginal extension with respect to $S_{0}, \ldots, S_{n}$.

Proof. By Theorem 3, it suffices to show that maximality satisfies sequential distributivity if and only if the given conditions hold.

"if". Consider any $1 \leq k<n$, any value $h_{k-1}$ of $H_{k-1}$, any finite set of gambles $\mathcal{X}$ on $S_{k}$, any finite sets of gambles $\mathcal{Y}\left(s_{k}\right)$ on $F_{k+1}$ (one such set for each $\left.s_{k} \in S_{k}\right)$, and any $X \in \mathcal{X}$ and $Y\left(s_{k}\right) \in \mathcal{Y}\left(s_{k}\right)$. We must show that

$$
\begin{aligned}
X+\bigoplus_{s_{k}} E_{s_{k}} Y\left(s_{k}\right) \in \operatorname{opt}\left(\mathcal{X}+\bigoplus_{s_{k}} E_{s_{k}} \mathcal{Y}\left(s_{k}\right) \mid h_{k-1}\right) \\
\Longleftrightarrow X \in \operatorname{opt}\left(\mathcal{X} \mid h_{k-1}\right) \text { and } Y\left(s_{k}\right) \in \operatorname{opt}\left(\mathcal{Y}\left(s_{k}\right) \mid h_{k-1} s_{k}\right) \text { for all } s_{k} .
\end{aligned}
$$

or, by the definition of maximality, that

$$
\begin{aligned}
\forall X^{\prime} \in \mathcal{X}, s_{k} \in & S_{k}, Y^{\prime}\left(s_{k}\right) \in \mathcal{Y}\left(s_{k}\right): \\
& \bar{P}\left(X+\bigoplus_{s_{k}} E_{s_{k}} Y\left(s_{k}\right)-X^{\prime}-\bigoplus_{s_{k}} E_{s_{k}} Y^{\prime}\left(s_{k}\right) \mid h_{k-1}\right) \geq 0
\end{aligned}
$$

is equivalent to

$$
\begin{gathered}
\forall X^{\prime} \in \mathcal{X}: \bar{P}\left(X-X^{\prime} \mid h_{k-1}\right) \geq 0, \text { and } \\
\forall s_{k}^{\prime} \in S_{k}, Y^{\prime \prime}\left(s_{k}^{\prime}\right) \in \mathcal{Y}\left(s_{k}^{\prime}\right): \bar{P}\left(Y\left(s_{k}^{\prime}\right)-Y^{\prime \prime}\left(s_{k}^{\prime}\right) \mid h_{k-1} s_{k}^{\prime}\right) \geq 0
\end{gathered}
$$

Obviously, Eq. (16) implies Eq. (17): simply consider the case in Eq. (16) where $Y^{\prime}\left(s_{k}\right)=Y\left(s_{k}\right)$ for all $s_{k}$.

Next, we show that Eq. (16) implies Eq. (18). Consider any $s_{k}^{\prime}$ and $Y^{\prime \prime}\left(s_{k}^{\prime}\right)$. In Eq. (16), take $X^{\prime}=X$, and $Y^{\prime}\left(s_{k}\right)=Y\left(s_{k}\right)$ for all $s_{k}$ except $s_{k}^{\prime}$, for which $Y^{\prime}\left(s_{k}^{\prime}\right)=Y^{\prime \prime}\left(s_{k}^{\prime}\right)$ :

$$
\begin{aligned}
0 & \leq \bar{P}\left(\bigoplus_{s_{k}} E_{s_{k}} Y\left(s_{k}\right)-\bigoplus_{s_{k}} E_{s_{k}} Y^{\prime}\left(s_{k}\right) \mid h_{k-1}\right) \\
& =\bar{P}\left(E_{s_{k}^{\prime}}\left(Y\left(s_{k}^{\prime}\right)-Y^{\prime \prime}\left(s_{k}^{\prime}\right)\right) \mid h_{k-1}\right)
\end{aligned}
$$

and by coherence [24, 6.3.5(5), p. 296],

$$
\leq \bar{P}\left(\bar{P}\left(E_{s_{k}^{\prime}}\left(Y\left(s_{k}^{\prime}\right)-Y^{\prime \prime}\left(s_{k}^{\prime}\right)\right) \mid h_{k-1} S_{k}\right) \mid h_{k-1}\right)
$$

and by separate coherence,

$$
=\bar{P}\left(E_{s_{k}^{\prime}} \bar{P}\left(Y\left(s_{k}^{\prime}\right)-Y^{\prime \prime}\left(s_{k}^{\prime}\right) \mid h_{k-1} s_{k}^{\prime}\right) \mid h_{k-1}\right)
$$


But, because $\underline{P}\left(E_{s_{k}^{\prime}} \mid h_{k-1}\right)>0$, the above inequality can only hold if

$$
\bar{P}\left(Y\left(s_{k}^{\prime}\right)-Y^{\prime \prime}\left(s_{k}^{\prime}\right) \mid h_{k-1} s_{k}^{\prime}\right) \geq 0
$$

which establishes the desired implication.

To complete the proof we show the opposite implication: if Eqs. (17) and (18) hold, will Eq. (16) hold too?

Consider any $X^{\prime} \in \mathcal{X}, s_{k} \in S_{k}$, and $Y^{\prime}\left(s_{k}\right) \in \mathcal{Y}\left(s_{k}\right)$. Then,

$$
\begin{aligned}
& \bar{P}\left(X+\bigoplus_{s_{k}} E_{s_{k}} Y\left(s_{k}\right)-X^{\prime}-\bigoplus_{s_{k}} E_{s_{k}} Y^{\prime}\left(s_{k}\right) \mid h_{k-1}\right) \\
& =\bar{P}\left(X-X^{\prime}+\bigoplus_{s_{k}} E_{s_{k}}\left(Y\left(s_{k}\right)-Y^{\prime}\left(s_{k}\right)\right) \mid h_{k-1}\right)
\end{aligned}
$$

and by assumption on $\underline{P}$,

$$
=\bar{P}\left(\bar{P}\left(X-X^{\prime}+\bigoplus_{s_{k}} E_{s_{k}}\left(Y\left(s_{k}\right)-Y^{\prime}\left(s_{k}\right)\right) \mid h_{k-1} S_{k}\right) \mid h_{k-1}\right)
$$

and by separate coherence,

$$
=\bar{P}\left(X-X^{\prime}+\bigoplus_{s_{k}} E_{s_{k}} \bar{P}\left(Y\left(s_{k}\right)-Y^{\prime}\left(s_{k}\right) \mid h_{k-1} s_{k}\right) \mid h_{k-1}\right)
$$

and by monotonicity of $\bar{P}$, and Eq. (18),

$$
\geq \bar{P}\left(X-X^{\prime} \mid h_{k-1}\right)
$$

and by Eq. (17),

$$
\geq 0 \text {. }
$$

"only if". We prove this part by contradiction. Assume $\underline{P}\left(E_{s_{k}^{\prime}} \mid h_{k-1}\right)=0$ for some $s_{k}^{\prime}$ and $h_{k-1}$. Consider the following sets of gambles:

$$
\begin{aligned}
\mathcal{X} & =\{0\} \\
\mathcal{Y}\left(s_{k}\right) & =\{0,1\} \text { for all } s_{k}
\end{aligned}
$$

Let $X=0$ and $Y\left(s_{k}\right)=1$ for all $s_{k}$ except $Y\left(s_{k}^{\prime}\right)=0$. Then

$$
X+\bigoplus_{s_{k}} E_{s_{k}} Y\left(s_{k}\right)=1-E_{s_{k}^{\prime}}
$$

obviously belongs to

$$
\operatorname{opt}\left(\mathcal{X}+\bigoplus_{s_{k}} E_{s_{k}} \mathcal{Y}\left(s_{k}\right) \mid h_{k-1}\right)
$$


because for any $X^{\prime} \in \mathcal{X}$ and $Y^{\prime}\left(s_{k}\right) \in \mathcal{Y}\left(s_{k}\right)$

$$
\begin{aligned}
& \bar{P}\left(X+\bigoplus_{s_{k}} E_{s_{k}} Y\left(s_{k}\right)-X^{\prime}-\bigoplus_{s_{k}} E_{s_{k}} Y^{\prime}\left(s_{k}\right) \mid h_{k-1}\right) \\
& =\bar{P}\left(\bigoplus_{s_{k}} E_{s_{k}}\left(Y\left(s_{k}\right)-Y^{\prime}\left(s_{k}\right)\right) \mid h_{k-1}\right)
\end{aligned}
$$

and by coherence $[24,2.6 .1(\mathrm{e}), \mathrm{p} .76]$,

$$
\geq \underline{P}\left(\bigoplus_{s_{k} \neq s_{k}^{\prime}} E_{s_{k}}\left(1-Y^{\prime}\left(s_{k}\right)\right) \mid h_{k-1}\right)+\bar{P}\left(-E_{s_{k}^{\prime}} Y^{\prime}\left(s_{k}\right) \mid h_{k-1}\right)
$$

which must be non-negative, because $1-Y^{\prime}\left(s_{k}\right)$ is non-negative for all $s_{k}$, and

$$
\left.\bar{P}\left(-E_{s_{k}^{\prime}} Y^{\prime}\left(s_{k}\right)\right) \mid h_{k-1}\right)=-Y^{\prime}\left(s_{k}\right) \underline{P}\left(E_{s_{k}^{\prime}} \mid h_{k-1}\right)=0 .
$$

But, $Y\left(s_{k}^{\prime}\right) \notin \operatorname{opt}\left(\mathcal{Y}\left(s_{k}^{\prime}\right) \mid h_{k-1} s_{k}^{\prime}\right)$, because

$$
\bar{P}\left(Y\left(s_{k}^{\prime}\right)-Y^{\prime \prime}\left(s_{k}^{\prime}\right) \mid h_{k-1} s_{k}^{\prime}\right)=-1 \nsupseteq 0
$$

for $Y^{\prime \prime}\left(s_{k}^{\prime}\right)=1$ : Property 2 fails. Hence, we have shown that $\underline{P}\left(E_{s_{k}} \mid h_{k-1}\right)>0$ must hold for Property 2 to hold as well.

Finally, assume that

$$
\underline{P}\left(Z \mid h_{k-1}\right)>\underline{P}\left(\underline{P}\left(Z \mid h_{k-1} S_{k}\right) \mid h_{k-1}\right) .
$$

for some gamble $Z$ and $h_{k-1}$ (indeed, if equality does not hold, we must have a strict inequality as given, by $[24,6.3 .5(5)$, p. 296]).

Consider the following sets of gambles:

$$
\begin{aligned}
\mathcal{X} & =\left\{\underline{P}\left(\underline{P}\left(Z \mid h_{k-1} S_{k}\right) \mid h_{k-1}\right), \underline{P}\left(Z \mid h_{k-1} S_{k}\right)\right\} \\
\mathcal{Y}\left(s_{k}\right) & =\left\{Z\left(s_{k}, \cdot\right), \underline{P}\left(Z \mid h_{k-1} s_{k}\right)\right\} \text { for all } s_{k}
\end{aligned}
$$

Let $X=\underline{P}\left(\underline{P}\left(Z \mid h_{k-1} S_{k}\right) \mid h_{k-1}\right)$ and $Y\left(s_{k}\right)=\underline{P}\left(Z \mid h_{k-1} s_{k}\right)$. Obviously, $X \in$ $\operatorname{opt}\left(\mathcal{X} \mid h_{k-1}\right)$ :

$$
\begin{aligned}
& \bar{P}\left(\underline{P}\left(\underline{P}\left(Z \mid h_{k-1} S_{k}\right) \mid h_{k-1}\right)-\underline{P}\left(Z \mid h_{k-1} S_{k}\right) \mid h_{k-1}\right) \\
& =\underline{P}\left(\underline{P}\left(Z \mid h_{k-1} S_{k}\right) \mid h_{k-1}\right)+\bar{P}\left(-\underline{P}\left(Z \mid h_{k-1} S_{k}\right) \mid h_{k-1}\right) \\
& =\underline{P}\left(\underline{P}\left(Z \mid h_{k-1} S_{k}\right) \mid h_{k-1}\right)-\underline{P}\left(\underline{P}\left(Z \mid h_{k-1} S_{k}\right) \mid h_{k-1}\right)=0
\end{aligned}
$$

If we can also show that

$$
Y\left(s_{k}\right) \in \operatorname{opt}\left(\mathcal{Y}\left(s_{k}\right) \mid h_{k-1} s_{k}\right) \text { for all } s_{k}
$$


but

$$
X+\bigoplus_{s_{k}} E_{s_{k}} Y\left(s_{k}\right) \notin \mathrm{opt}\left(\mathcal{X}+\bigoplus_{s_{k}} E_{s_{k}} \mathcal{Y}\left(s_{k}\right) \mid h_{k-1}\right)
$$

then it is established that Property 2 cannot hold.

Indeed, for any $Y^{\prime \prime}\left(s_{k}\right) \in \mathcal{Y}\left(s_{k}\right)$,

$$
\begin{aligned}
\bar{P}\left(Y\left(s_{k}\right)-Y^{\prime \prime}\left(s_{k}\right) \mid h_{k-1} s_{k}\right) & =\bar{P}\left(\underline{P}\left(Z \mid h_{k-1} s_{k}\right)-Y^{\prime \prime}\left(s_{k}\right) \mid h_{k-1} s_{k}\right) \\
& =\underline{P}\left(Z \mid h_{k-1} s_{k}\right)+\bar{P}\left(-Y^{\prime \prime}\left(s_{k}\right) \mid h_{k-1} s_{k}\right) \\
& =\underline{P}\left(Z \mid h_{k-1} s_{k}\right)-\underline{P}\left(Y^{\prime \prime}\left(s_{k}\right) \mid h_{k-1} s_{k}\right)
\end{aligned}
$$

but, $Y^{\prime \prime}\left(s_{k}\right)$ is either $\underline{P}\left(Z \mid h_{k-1} s_{k}\right)$ or $Z\left(s_{k}, \cdot\right)$, and in either case,

$$
=0 \text {. }
$$

which shows that Eq. (19) is satisfied.

However, for $X^{\prime}=\underline{P}\left(Z \mid h_{k-1} S_{k}\right)$ and $Y^{\prime}\left(s_{k}\right)=Z\left(s_{k}, \cdot\right)$, we have that

$$
\begin{aligned}
& \underline{P}\left(X^{\prime}+\bigoplus_{s_{k}} E_{s_{k}} Y^{\prime}\left(s_{k}\right)-X-\bigoplus_{s_{k}} E_{s_{k}} Y\left(s_{k}\right) \mid h_{k-1}\right) \\
& =\underline{P}\left(\underline{P}\left(Z \mid h_{k-1} S_{k}\right)+Z-\underline{P}\left(\underline{P}\left(Z \mid h_{k-1} S_{k}\right) \mid h_{k-1}\right)-\underline{P}\left(Z \mid h_{k-1} S_{k}\right) \mid h_{k-1}\right) \\
& =\underline{P}\left(Z \mid h_{k-1}\right)-\underline{P}\left(\underline{P}\left(Z \mid h_{k-1} S_{k}\right) \mid h_{k-1}\right)>0
\end{aligned}
$$

which means that Eq. (20) is satisfied.

A first observation is that locality provides a behavioural argument for marginal extension: violating marginal extension with respect to some sequence of states $S_{0}, \ldots, S_{n}$, then you must violate locality for some act-state independent sequential decision problems on $S_{0}, \ldots, S_{n}$. Although marginal extension is a convenient assumption to make, for instance due to computational reasons $[24, \S 6.7 .5$, p. 316], we are not aware of any other behavioural motivation.

Secondly, one can easily see that the condition of strictly positive lower probability can be relaxed quite easily through perturbation. Indeed, it is well known that a small perturbation of a coherent lower prevision does not affect optimality much [23]. Hence, for a coherent lower prevision $\underline{P}$ which satisfies marginal extension but has some zero lower probability events, we can consider the local models

$$
\underline{P}_{\epsilon}\left(\cdot \mid h_{k-1}\right)=(1-\epsilon) \underline{P}\left(\cdot \mid h_{k-1}\right)+\epsilon Q\left(\cdot \mid h_{k-1}\right)
$$

on gambles on $S_{k}$, where $Q$ is a linear prevision (i.e. expectation) which has all probabilities strictly positive - for example, the conditional expectation with respect to the uniform mass function. The local models $\underline{P}_{\epsilon}$ can be combined to a global model via marginal extension:

$$
\underline{P}_{\epsilon}\left(Z \mid h_{k-1}\right)=\underline{P}_{\epsilon}\left(\underline{P}_{\epsilon}\left(Z \mid h_{k-1} S_{k}\right) \mid h_{k-1}\right)
$$

for any $1 \leq k<n$ and any gamble $Z$ on $S_{k} \times \ldots S_{n}$. We observe then that: 
- Maximality with respect to $\underline{P}_{\epsilon}$ satisfies locality, because $\underline{P}_{\epsilon}$ now has strictly positive lower probabilities, and satisfies marginal extension by construction.

- For small $\epsilon>0$, maximality with respect to $\underline{P}_{\epsilon}$ is almost identical to maximality with respect to $\underline{P}$, because the global model $\underline{P}_{\epsilon}\left(\cdot \mid h_{k-1}\right)$ is still within distance $(n-k+1) \epsilon$ of the global model $\underline{P}$ : for any $0<k \leq n$ and any gamble $Z$ on $S_{k} \times \ldots S_{n},{ }^{1}$

$$
\left|\underline{P}_{\epsilon}\left(Z \mid h_{k-1}\right)-\underline{P}\left(Z \mid h_{k-1}\right)\right| \leq(n-k+1) \epsilon \sup |Z|
$$

Hence, for small $\epsilon>0$, the local solution to our decision problem with respect to $\underline{P}$ approximately coincides with the local (and hence, also global!) solution with respect to $\underline{P}_{\epsilon}$. This shows that solving problems locally, even if lower probabilities are zero, is not at all a silly thing to do, as you will still be (almost) optimal with respect to any close approximation of your original assessments (as long as this approximation has strictly positive lower probabilities). More specifically, if opt $\epsilon_{\epsilon}^{\delta}$ denotes $\delta$-maximality ${ }^{2}$ with respect to $\underline{P}_{\epsilon}$, then

$$
\operatorname{opt}_{\epsilon}^{0}\left(\mathcal{Z} \mid h_{k-1}\right) \subseteq \operatorname{opt}_{0}^{2(n-k+1) \epsilon}\left(\mathcal{Z} \mid h_{k-1}\right) \subseteq \operatorname{opt}_{\epsilon}^{4(n-k+1) \epsilon}\left(\mathcal{Z} \mid h_{k-1}\right)
$$

for any set of gambles $\mathcal{Z}$ [23, p. 664]. So, locally, you do not need to be concerned about zero lower probabilities, if you are willing to accept an (arbitrarily close) approximate version of maximality.

In our toy example, first, our agent must assess a coherent lower prevision $\underline{P}(\cdot \mid \cdot)$ reflecting his beliefs about the coin. For instance, he could use the imprecise Dirichlet model [25], which states that, for any gamble $X$ on $S_{k}=\{H, T\}$ :

$$
\underline{P}\left(X \mid h_{k-1}\right)=\frac{n_{H} X(H)+n_{T} X(T)+s \min \{X(H), X(T)\}}{n_{H}+n_{T}+s}
$$

where $n_{H}$ is the number of heads observed in $h_{k-1}, n_{T}$ is the number of tails in $h_{k-1}$, and $s$ is a hyper-parameter of the model, usually taken to be 1 or 2 . Eq. (21) is called the predictive lower prevision. Similarly, the predictive upper prevision is

$$
\bar{P}\left(X \mid h_{k-1}\right)=\frac{n_{H} X(H)+n_{T} X(T)+s \max \{X(H), X(T)\}}{n_{H}+n_{T}+s}
$$

The IDM models a completely vacuous state of knowledge if $n_{H}=n_{T}=0$, and converges to the empirical expectation as $n_{H}+n_{T}$ grows, hence this seems a reasonable model.

The predictive lower previsions yield marginals on gambles on $S_{k}$ conditional on $h_{k-1}$. The most conservative joint lower prevision that is compatible with

\footnotetext{
${ }^{1}$ This is not the tightest possible bound, but it is easily derived by induction on $k$ (start at $k=n)$ and suffices to make our argument.

${ }^{2} Z$ is $\delta$-maximal in $\mathcal{Z}$ with respect to $\underline{P}$ if $\bar{P}\left(Z-Z^{\prime}\right) \geq-\delta \sup _{Z^{\prime} \in \mathcal{Z}}\left|Z^{\prime}\right|$ for all $Z^{\prime} \in \mathcal{Z}$.
} 
these marginals is given by repeatedly applying the marginal extension theorem $[24$, p. $314-315, \S 6.7 .2]$ : for any gamble $Z$ on $S_{k} \times \cdots \times S_{n}$, define

$$
\underline{P}\left(Z \mid h_{k-1}\right)=\underline{P}\left(\underline{P}\left(Z \mid h_{k-1} S_{k}\right) \mid h_{k-1}\right)
$$

(where $\underline{P}\left(Z \mid h_{k-1} S_{k}\right)$ is considered as a gamble on $S_{k}$ ).

Applying maximality to our toy example is now straightforward. By definition of maximality (Eq. (15)), betting on heads is locally maximal if

$$
\bar{P}\left(X_{k}^{k}\left(h_{k-1}, d_{H}\right)-X_{k}^{k}\left(h_{k-1}, d_{T}\right) \mid h_{k-1}\right) \geq 0 .
$$

By $X_{k}^{k}\left(h_{k-1}, d_{H}\right)=-X_{k}^{k}\left(h_{k-1}, d_{T}\right)$, and Eqs. (7) and (22), we conclude that, locally, betting on heads is optimal whenever $n_{H} \geq n_{T}+s$, and similarly, betting on tails is optimal whenever $n_{T} \geq n_{H}+s$. By construction, $\underline{P}(\cdot \mid \cdot)$ satisfies marginal extension, so applying Proposition 6, we conclude that this is also the global solution (after small perturbation to get rid of any zero lower probabilities).

\section{$5.2 \quad$ E-admissibility}

Given a full conditional expectation $Q(\cdot \mid \cdot)$, let $\operatorname{opt}_{Q}(\cdot \mid A)$ be the choice function corresponding to maximising expected utility conditionally on $A$, and let $\mathcal{M}(\cdot \mid A)$ denote the set of full conditional expectations that dominate a given coherent lower prevision $\underline{P}(\cdot \mid A)$. Then E-admissibility with respect to $\underline{P}(\cdot \mid A)$ is:

$$
\operatorname{opt}(\mathcal{X} \mid A)=\bigcup_{Q(\cdot \mid A) \in \mathcal{M}(\cdot \mid A)} \operatorname{opt}_{Q}(\mathcal{X} \mid A) .
$$

Perhaps surprisingly, the conditions are identical to those for maximality:

Proposition 7. E-admissibility satisfies locality on $S_{0}, \ldots, S_{n}$ if and only if

(i) $\underline{P}\left(E_{s_{k}} \mid h_{k-1}\right)>0$ for all $1 \leq k<n, h_{k-1} \in H_{k-1}$, and $s_{k} \in S_{k}$, and

(ii) $\underline{P}$ satisfies marginal extension with respect to $S_{0}, \ldots, S_{n}$.

Proof. "if". If

$$
X+\bigoplus_{s_{k}} E_{s_{k}} Y\left(s_{k}\right) \in \operatorname{opt}\left(\mathcal{X}+\bigoplus_{s_{k}} E_{s_{k}} \mathcal{Y}\left(s_{k}\right) \mid h_{k-1}\right),
$$

then, by definition of E-admissibility, there is a $Q \in \mathcal{M}$ such that

$$
X+\bigoplus_{s_{k}} E_{s_{k}} Y\left(s_{k}\right) \in \operatorname{opt}_{Q}\left(\mathcal{X}+\bigoplus_{s_{k}} E_{s_{k}} \mathcal{Y}\left(s_{k}\right) \mid h_{k-1}\right)
$$

Since we assumed that $\underline{P}\left(E_{s_{k}} \mid h_{k-1}\right)>0$, we know that $Q\left(E_{s_{k}} \mid h_{k-1}\right)>0$. Given this, and Bayes theorem for linear previsions, it follows easily from Proposition 6 that Property 2 holds for maximality with respect to $Q$. Therefore,

$$
X \in \operatorname{opt}_{Q}\left(\mathcal{X} \mid h_{k-1}\right) \text { and } Y\left(s_{k}\right) \in \operatorname{opt}_{Q}\left(\mathcal{Y}\left(s_{k}\right) \mid h_{k-1} s_{k}\right) \text { for all } s_{k} .
$$


and so, again by definition of E-admissibility,

$X \in \operatorname{opt}\left(\mathcal{X} \mid h_{k-1}\right)$ and $Y\left(s_{k}\right) \in \operatorname{opt}\left(\mathcal{Y}\left(s_{k}\right) \mid h_{k-1} s_{k}\right)$ for all $s_{k}$.

We have proved one direction of Property 2.

Let us now prove the other direction. If

$X \in \operatorname{opt}\left(\mathcal{X} \mid h_{k-1}\right)$ and $Y\left(s_{k}\right) \in \operatorname{opt}\left(\mathcal{Y}\left(s_{k}\right) \mid h_{k-1} s_{k}\right)$ for all $s_{k}$,

then, there are linear previsions $Q\left(\cdot \mid h_{k-1}\right) \in \mathcal{M}\left(\cdot \mid h_{k-1}\right)$ and $R\left(\cdot \mid h_{k-1} s_{k}\right) \in$ $\mathcal{M}\left(\cdot \mid h_{k-1} s_{k}\right)$ for all $s_{k}$ such that

$X \in \operatorname{opt}_{Q}\left(\mathcal{X} \mid h_{k-1}\right)$ and $Y\left(s_{k}\right) \in \operatorname{opt}_{R}\left(\mathcal{Y}\left(s_{k}\right) \mid h_{k-1} s_{k}\right)$ for all $s_{k}$.

By the lower envelope theorem for marginal extension [24, 6.7.4], the linear prevision $Q$

$$
Q\left(\cdot \mid h_{k-1}\right)=Q\left(R\left(\cdot \mid h_{k-1} S_{k}\right) \mid h_{k-1}\right)
$$

belongs to $\mathcal{M}\left(\cdot \mid h_{k-1}\right)$. But, using the linearity of $Q$, Bayes theorem, and the above decomposition of $Q$,

$$
\begin{aligned}
& \max _{\substack{X^{\prime} \in \mathcal{X} \\
Y^{\prime}\left(s_{k}\right) \in \mathcal{Y}\left(s_{k}\right)}} Q\left(X^{\prime}+\bigoplus_{s_{k}} E_{s_{k}} Y^{\prime}\left(s_{k}\right) \mid h_{k-1}\right) \\
= & \max _{X^{\prime} \in \mathcal{X}} Q\left(X^{\prime} \mid h_{k-1}\right)+\sum_{s_{k}} Q\left(E_{s_{k}} \mid h_{k-1}\right) \max _{Y^{\prime}\left(s_{k}\right) \in \mathcal{Y}\left(s_{k}\right)} Q\left(Y^{\prime}\left(s_{k}\right) \mid h_{k-1} s_{k}\right) \\
= & \max _{X^{\prime} \in \mathcal{X}} Q\left(X^{\prime} \mid h_{k-1}\right)+\sum_{s_{k}} Q\left(E_{s_{k}} \mid h_{k-1}\right) \max _{Y^{\prime}\left(s_{k}\right) \in \mathcal{Y}\left(s_{k}\right)} R\left(Y^{\prime}\left(s_{k}\right) \mid h_{k-1} s_{k}\right) \\
= & Q\left(X \mid h_{k-1}\right)+\sum_{s_{k}} Q\left(E_{s_{k}} \mid h_{k-1}\right) R\left(Y\left(s_{k}\right) \mid h_{k-1} s_{k}\right) \\
= & Q\left(X \mid h_{k-1}\right)+\sum_{s_{k}} Q\left(E_{s_{k}} \mid h_{k-1}\right) Q\left(Y\left(s_{k}\right) \mid h_{k-1} s_{k}\right) \\
= & Q\left(X+\bigoplus_{s_{k}} E_{s_{k}} Y\left(s_{k}\right) \mid h_{k-1}\right)
\end{aligned}
$$

so,

$$
X+\bigoplus_{s_{k}} E_{s_{k}} Y\left(s_{k}\right) \in \operatorname{opt}_{Q}\left(\mathcal{X}+\bigoplus_{s_{k}} E_{s_{k}} \mathcal{Y}\left(s_{k}\right) \mid h_{k-1}\right)
$$

whence

$$
X+\bigoplus_{s_{k}} E_{s_{k}} Y\left(s_{k}\right) \in \operatorname{opt}\left(\mathcal{X}+\bigoplus_{s_{k}} E_{s_{k}} \mathcal{Y}\left(s_{k}\right) \mid h_{k-1}\right) .
$$

"only if". Assume $\underline{P}\left(E_{s_{k}^{\prime}} \mid h_{k-1}\right)=0$ for some $s_{k}^{\prime}$ and $h_{k-1}$. Then, there is a $Q \in \mathcal{M}$ such that $Q\left(E_{s_{k}^{\prime}} \mid h_{k-1}\right)=0$. Consider the following sets of gambles:

$$
\begin{aligned}
\mathcal{X} & =\{0\} \\
\mathcal{Y}\left(s_{k}\right) & =\{0,1\} \text { for all } s_{k}
\end{aligned}
$$


Let $X=0$ and $Y\left(s_{k}\right)=1$ for all $s_{k}$ except $Y\left(s_{k}^{\prime}\right)=0$. Then

$$
X+\bigoplus_{s_{k}} E_{s_{k}} Y\left(s_{k}\right)=1-E_{s_{k}^{\prime}}
$$

belongs to

$$
\operatorname{opt}\left(\mathcal{X}+\bigoplus_{s_{k}} E_{s_{k}} \mathcal{Y}\left(s_{k}\right) \mid h_{k-1}\right)
$$

because $Q\left(1-E_{s_{k}^{\prime}} \mid h_{k-1}\right)=1$ and of course there is no gamble in $\mathcal{X}+\bigoplus_{s_{k}} E_{s_{k}} \mathcal{Y}\left(s_{k}\right)$ with expectation greater than 1 . But clearly for any $s_{k}$, opt $\left(\mathcal{Y}\left(s_{k}\right) \mid h_{k-1} s_{k}\right)=$ $\{1\}$ and so $Y\left(s_{k}^{\prime}\right) \notin \operatorname{opt}\left(\mathcal{Y}\left(s_{k}^{\prime}\right) \mid h_{k-1} s_{k}^{\prime}\right)=\{1\}$. Therefore if $\underline{P}\left(E_{s_{k}^{\prime}} \mid h_{k-1}\right)=0$ for some $s_{k}^{\prime}$ and $h_{k-1}$, Property 2 cannot hold.

Finally, assume that

$$
\underline{P}\left(Z \mid h_{k-1}\right)>\underline{P}\left(\underline{P}\left(Z \mid h_{k-1} S_{k}\right) \mid h_{k-1}\right) .
$$

for some gamble $Z$ and $h_{k-1}$ (indeed, if equality does not hold, we must have a strict inequality as given, by $[24,6.3 .5(5)$, p. 296]).

Consider the sets of gambles used in the maximality proof:

$$
\begin{aligned}
\mathcal{X} & =\left\{\underline{P}\left(\underline{P}\left(Z \mid h_{k-1} S_{k}\right) \mid h_{k-1}\right), \underline{P}\left(Z \mid h_{k-1} S_{k}\right)\right\} \\
\mathcal{Y}\left(s_{k}\right) & =\left\{Z\left(s_{k}, \cdot\right), \underline{P}\left(Z \mid h_{k-1} s_{k}\right)\right\} \text { for all } s_{k}
\end{aligned}
$$

Let $X=\underline{P}\left(\underline{P}\left(Z \mid h_{k-1} S_{k}\right) \mid h_{k-1}\right)$ and $Y\left(s_{k}\right)=\underline{P}\left(Z \mid h_{k-1} s_{k}\right)$. When a set of gambles has only two elements, E-admissibility and maximality coincide. So we know from the previous proof that, for E-admissibility,

$$
\begin{aligned}
X & \in \operatorname{opt}\left(\mathcal{X} \mid h_{k-1}\right) \\
Y\left(s_{k}\right) & \in \operatorname{opt}\left(\mathcal{Y}\left(s_{k}\right) \mid h_{k-1} s_{k}\right) \text { for all } s_{k} .
\end{aligned}
$$

The set of E-admissible gambles is always a subset of the maximal gambles, so if a gamble is non-maximal in a set, it is not E-admissible either. In the proof for maximality, we showed that

$$
X+\bigoplus_{s_{k}} E_{s_{k}} Y\left(s_{k}\right)
$$

is not maximal in

$$
\mathcal{X}+\bigoplus_{s_{k}} E_{s_{k}} \mathcal{Y}\left(s_{k}\right),
$$

and so it is not E-admissible. Hence, again, Property 2 does not hold.

In order to apply E-admissibility to the example, simply note that E-admissibility is equivalent to maximality in case of binary choice: indeed, a gamble $X$ is maximal in $\{X, Y\}$ if and only if

$$
\bar{P}(X-Y) \geq 0
$$


which is, by the lower envelope theorem [24, p. 134, §3.3.3], equivalent to

$$
\exists Q \in \mathcal{M}: Q(X-Y) \geq 0
$$

which means exactly that $X$ is E-admissible in $\{X, Y\}$.

Hence, locally, E-admissibility and maximality coincide and the agent always selects the same action in both cases. So, by Proposition 7, whose conditions we already verified earlier, E-admissibility and maximality also coincide globally.

\section{$5.3 \Gamma$-maximin}

$\Gamma$-maximin with respect to a coherent lower prevision $\underline{P}$ is the choice function

$$
\operatorname{opt}(\mathcal{X} \mid A)=\arg \max _{X \in \mathcal{X}} \underline{P}(X \mid A) \text {. }
$$

Proposition 8. $\Gamma$-maximin with respect to a coherent lower prevision $\underline{P}$ satisfies locality on $S_{0}, \ldots, S_{n}$ if and only if the following three conditions hold:

(i) $\underline{P}\left(E_{s_{k}} \mid h_{k-1}\right)>0$ for all $1 \leq k<n, h_{k-1} \in H_{k-1}$, and $s_{k} \in S_{k}$,

(ii) $\underline{P}$ satisfies marginal extension with respect to $S_{0}, \ldots, S_{n}$, and

(iii) $\underline{P}$ is locally linear in the sense that, for all $1 \leq k<n$ and all gambles $X$ and $Y$ on $S_{k}$,

$$
\underline{P}\left(X+Y \mid h_{k-1}\right)=\underline{P}\left(X \mid h_{k-1}\right)+\underline{P}\left(Y \mid h_{k-1}\right),
$$

Proof. "if". Consider any $1 \leq k<n$, any value $h_{k-1}$ of $H_{k-1}$, any finite set of gambles $\mathcal{X}$ on $S_{k}$, any finite sets of gambles $\mathcal{Y}\left(s_{k}\right)$ on $F_{k+1}$ (one such set for each $\left.s_{k} \in S_{k}\right)$, and any $X \in \mathcal{X}$ and $Y\left(s_{k}\right) \in \mathcal{Y}\left(s_{k}\right)$. We must show that

$$
\begin{aligned}
X+ & \bigoplus_{s_{k}} E_{s_{k}} Y\left(s_{k}\right) \in \operatorname{opt}\left(\mathcal{X}+\bigoplus_{s_{k}} E_{s_{k}} \mathcal{Y}\left(s_{k}\right) \mid h_{k-1}\right) \\
& \Longleftrightarrow X \in \operatorname{opt}\left(\mathcal{X} \mid h_{k-1}\right) \text { and } Y\left(s_{k}\right) \in \operatorname{opt}\left(\mathcal{Y}\left(s_{k}\right) \mid h_{k-1} s_{k}\right) \text { for all } s_{k}
\end{aligned}
$$

We have, by marginal extension,

$$
\begin{aligned}
& \underline{P}\left(X+\bigoplus_{s_{k}} E_{s_{k}} Y\left(s_{k}\right) \mid h_{k-1}\right) \\
& =\underline{P}\left(X+\bigoplus_{s_{k}} E_{s_{k}} \underline{P}\left(Y\left(s_{k}\right) \mid h_{k-1} s_{k}\right) \mid h_{k-1}\right)
\end{aligned}
$$

whence, by local linearity

$$
=\underline{P}\left(X \mid h_{k-1}\right)+\underline{P}\left(\bigoplus E_{s_{k}} \underline{P}\left(Y\left(s_{k}\right) \mid h_{k-1} s_{k}\right) \mid h_{k-1}\right) .
$$


This expression can clearly be maximised by choosing $X$ to maximise $\underline{P}\left(X \mid h_{k-1}\right)$ and choosing each $Y\left(s_{k}\right)$ to maximise $\underline{P}\left(Y\left(s_{k}\right) \mid h_{k-1} s_{k}\right)$. That is, if $X \in \operatorname{opt}\left(\mathcal{X} \mid h_{k-1}\right)$ and $Y\left(s_{k}\right) \in \operatorname{opt}\left(\mathcal{Y}\left(s_{k}\right) \mid h_{k-1} s_{k}\right)$ for all $s_{k}$, then Eq. (24) is maximal. This establishes the left implication in Eq. (23).

Next, suppose that

$$
X+\bigoplus_{s_{k}} E_{s_{k}} Y\left(s_{k}\right) \in \operatorname{opt}\left(\mathcal{X}+\bigoplus_{s_{k}} E_{s_{k}} \mathcal{Y}\left(s_{k}\right) \mid h_{k-1}\right),
$$

so there is no other gamble in $\mathcal{X}+\bigoplus_{s_{k}} E_{s_{k}} \mathcal{Y}\left(s_{k}\right)$ with a higher lower prevision. Again considering Eq. (24), it is clear that $X \in \operatorname{opt}\left(\mathcal{X} \mid h_{k-1}\right)$, since otherwise we could increase the lower prevision by instead using an optimal element of $\mathcal{X}$. Also, because $\underline{P}\left(E_{s_{k}} \mid h_{k-1}\right)>0$, we see that $Y\left(s_{k}\right) \in \operatorname{opt}\left(\mathcal{Y}\left(s_{k}\right) \mid h_{k-1} s_{k}\right)$, form otherwise we could increase the lower prevision by using an optimal element of $\mathcal{Y}\left(s_{k}\right)$. This establishes the right implication in Eq. (23).

"only if". Suppose that $\underline{P}\left(\cdot \mid h_{k-1}\right)$ is not locally linear for some $h_{k-1} \in H_{k-1}$. Then there must be gambles $X$ and $Y$ on $S_{k}$, and an $\epsilon>0$, such that

$$
\underline{P}\left(X \mid h_{k-1}\right)+\underline{P}\left(Y \mid h_{k-1}\right)+\epsilon<\underline{P}\left(X+Y \mid h_{k-1}\right) .
$$

Let $\mathcal{X}=\left\{X, \underline{P}\left(X \mid h_{k-1}\right)+\epsilon\right\}$ and $\mathcal{Y}\left(s_{k}\right)=\left\{Y\left(s_{k}\right)\right\}$. Obviously, opt $\left(\mathcal{X} \mid h_{k-1}\right)=$ $\left\{\underline{P}\left(X \mid h_{k-1}\right)+\epsilon\right\}$, and $\operatorname{opt}\left(\mathcal{Y}\left(s_{k}\right) \mid h_{k-1}\right)=\left\{Y\left(s_{k}\right)\right\}$. However,

$$
\begin{aligned}
\operatorname{opt}\left(\mathcal{X}+\bigoplus_{s_{k}} E_{s_{k}} \mathcal{Y}\left(s_{k}\right) \mid h_{k-1}\right) & =\operatorname{opt}\left(\left\{X+Y, \underline{P}\left(X \mid h_{k-1}\right)+\epsilon+Y\right\} \mid h_{k-1}\right) \\
& =\{X+Y\}
\end{aligned}
$$

because

$$
\begin{aligned}
\underline{P}\left(\underline{P}\left(X \mid h_{k-1}\right)+\epsilon+Y \mid h_{k-1}\right) & =\underline{P}\left(X \mid h_{k-1}\right)+\epsilon+\underline{P}\left(Y \mid h_{k-1}\right) \\
& <\underline{P}\left(X+Y \mid h_{k-1}\right) .
\end{aligned}
$$

So, locality fails whenever local linearity fails.

Next, we show that marginal extension must hold. Suppose it does not, and hence that

$$
\underline{P}\left(Z \mid h_{k-1}\right)>\underline{P}\left(\underline{P}\left(Z \mid h_{k-1} S_{k}\right) \mid h_{k-1}\right) .
$$

for some gamble $Z$ and $h_{k-1}$.

Consider the following sets of gambles:

$$
\begin{aligned}
\mathcal{X} & =\left\{\underline{P}\left(\underline{P}\left(Z \mid h_{k-1} S_{k}\right) \mid h_{k-1}\right), \underline{P}\left(Z \mid h_{k-1} S_{k}\right)\right\} \\
\mathcal{Y}\left(s_{k}\right) & =\left\{Z\left(s_{k}, \cdot\right), \underline{P}\left(Z \mid h_{k-1} s_{k}\right)\right\} \text { for all } s_{k}
\end{aligned}
$$

Let $X=\underline{P}\left(\underline{P}\left(Z \mid h_{k-1} S_{k}\right) \mid h_{k-1}\right)$ and $Y\left(s_{k}\right)=\underline{P}\left(Z \mid h_{k-1} s_{k}\right)$. It is immediate that $\operatorname{opt}\left(\mathcal{X} \mid h_{k-1}\right)=\mathcal{X}$ and $\operatorname{opt}\left(\mathcal{Y}\left(s_{k}\right) \mid h_{k-1} s_{k}\right)=\bar{Y}\left(s_{k}\right)$. But as we saw in the proof of Proposition 6, the gamble $X+\bigoplus_{s_{k}} E_{s_{k}} Y\left(s_{k}\right)$ is not maximal. A $\Gamma$-maximin 
gamble is always maximal [24, 3.9.7], and therefore $X+\bigoplus_{s_{k}} E_{s_{k}} Y\left(s_{k}\right)$ is not $\Gamma$-maximin either, and so locality fails.

Finally, assume $\underline{P}\left(E_{s_{k}^{\prime}} \mid h_{k-1}\right)=0$ for some $s_{k}^{\prime}$ and $h_{k-1}$. Consider the following sets of gambles:

$$
\begin{aligned}
\mathcal{X} & =\{0\} \\
\mathcal{Y}\left(s_{k}\right) & =\{0,1\} \text { for all } s_{k}
\end{aligned}
$$

Let $X=0$ and $Y\left(s_{k}\right)=1$ for all $s_{k}$ except $Y\left(s_{k}^{\prime}\right)=0$. Then

$$
X+\bigoplus_{s_{k}} E_{s_{k}} Y\left(s_{k}\right)=1-E_{s_{k}^{\prime}}
$$

and this gamble has lower prevision 1 , which is certainly no smaller than that of any other gamble, and so this gamble is $\Gamma$-maximin. But it is also clear that $Y\left(s_{k}^{\prime}\right)$ is not in $\operatorname{opt}\left(\mathcal{Y}\left(s_{k}^{\prime}\right) \mid h_{k-1} s_{k}\right)$, and so locality fails.

The locality conditions for $\Gamma$-maximin imply full linearity on all gambles on $S_{0} \times \cdots \times S_{n-1}$, i.e. linearity on every gamble that does not involve the final state $S_{n}$. Of course, in cases where such strong form of linearity is satisfied, usually full linearity will actually be satisfied. In other words, one cannot really endorse locality for $\Gamma$-maximin and at the same time use imprecise probabilities.

Corollary 9. If $\Gamma$-maximin satisfies locality, then for any $1 \leq k<n$, any gamble $Z$ on $S_{k} \times \ldots S_{n-1}$, and any $h_{k-1} \in H_{k-1}$,

$$
\underline{P}\left(Z \mid h_{k-1}\right)=\bar{P}\left(Z \mid h_{k-1}\right) .
$$

Proof. Simply apply local linearity and marginal extension repeatedly. Indeed, the case $k=n-1$ follows from local linearity. Suppose that we have already established the result for $k=m+1$, let us show that it also holds for $k=m$. For any gamble $Z$ on $S_{m} \times \cdots \times S_{n-1}$, we have, by marginal extension

$$
\underline{P}\left(Z \mid h_{m-1}\right)=\underline{P}\left(\underline{P}\left(Z \mid h_{m-1} S_{m}\right) \mid h_{m-1}\right)
$$

but, $\underline{P}\left(Z \mid h_{m-1} S_{m}\right)$ is a gamble on $S_{m}$, and hence, by local linearity,

$$
=\bar{P}\left(\underline{P}\left(Z \mid h_{m-1} S_{m}\right) \mid h_{m-1}\right)
$$

but, by the induction hypothesis, $\underline{P}\left(\cdot \mid h_{m-1} s_{m}\right)$ is linear for all $s_{m} \in S_{m}$, and hence,

$$
=\bar{P}\left(\bar{P}\left(Z \mid h_{m-1} S_{m}\right) \mid h_{m-1}\right)
$$

and hence, again by marginal extension,

$$
=\bar{P}\left(Z \mid h_{m-1}\right)
$$

which establishes the desired result. 
However, a locally $\Gamma$-maximin policy is always locally maximal, and so if marginal extension holds it is globally maximal, by Proposition 6. So, using a locally $\Gamma$-maximin policy may be a reasonable choice, even though it is not always globally $\Gamma$-maximin.

In our example, betting on heads is optimal under local $\Gamma$-maximin whenever

$$
\underline{P}\left(X_{k}^{k}\left(h_{k-1}, d_{H}\right) \mid h_{k-1}\right) \geq \underline{P}\left(X_{k}^{k}\left(h_{k-1}, d_{T}\right) \mid h_{k-1}\right) .
$$

By Eqs. (7) and (21), we obtain

$$
n_{H} \geq n_{T} \text {. }
$$

So, betting on heads is locally optimal if $n_{H} \geq n_{T}$ and similarly on tails when $n_{T} \geq n_{H}$. However, by Proposition 8 , for this also to be a global $\Gamma$-maximin policy, $\underline{P}$ would be required to be a linear prevision (except on the last stage). Because the imprecise Dirichlet model starts with a vacuous lower prevision as prior, $\underline{P}$ does not satisfy the linearity condition, and hence, the policy we just found is not necessarily globally $\Gamma$-maximin. Nevertheless, the local $\Gamma$-maximin policy is still an interesting alternative for the reasons we highlighted earlier.

\subsection{Interval Dominance}

Interval dominance with respect to a coherent lower prevision $\underline{P}$ is:

$$
\operatorname{opt}(\mathcal{X} \mid A)=\{X \in \mathcal{X}:(\forall Y \in \mathcal{X})(\bar{P}(X \mid A) \geq \underline{P}(Y \mid A))\} .
$$

Proposition 10. Interval dominance with respect to a coherent lower prevision $\underline{P}$ satisfies locality on $S_{0}, \ldots, S_{n}$ if and only if

(i) $\underline{P}\left(E_{s_{k}} \mid h_{k-1}\right)>0$ for all $1 \leq k<n, h_{k-1} \in H_{k-1}$, and $s_{k} \in S_{k}$,

(ii) $\underline{P}$ satisfies marginal extension with respect to $S_{0}, \ldots, S_{n}$, and

(iii) $\underline{P}\left(\cdot \mid h_{k-1}\right)$ is locally linear, in the sense that for all $1 \leq k \leq n, h_{k-1} \in$ $H_{k-1}$, and gambles $X$ on $S_{k}$,

$$
\underline{P}\left(X \mid h_{k-1}\right)=\bar{P}\left(X \mid h_{k-1}\right)
$$

Proof. "if". If $\underline{P}$ satisfies properties (ii) and (iii), then it is easy to show that $\underline{P}\left(\cdot \mid h_{k-1}\right)$ is linear for any $1 \leq k \leq n$ and all gambles on $S_{k} \times \cdots \times S_{n}$ (see the proof of Corollary 11). This implies that interval dominance and maximality coincide at every stage, and so by Property (i) and Proposition 6, interval dominance satisfies locality.

"only if". Suppose first that $\underline{P}\left(\cdot \mid h_{k-1}\right)$ is not locally linear for some $1 \leq k<$ $n$ and $h_{k-1} \in H_{k-1}$. Then there must be a gamble $X$ on $S_{k}$ and an $\epsilon>0$ such that

$$
\underline{P}\left(X \mid h_{k}\right)+\epsilon<\bar{P}\left(X \mid h_{k}\right) .
$$


Let $\mathcal{X}=\{0\}$ and $\mathcal{Y}\left(s_{k}\right)=\left\{X\left(s_{k}\right), X\left(s_{k}\right)+\epsilon\right\}$. Obviously, opt $\left(\mathcal{X} \mid h_{k-1}\right)=$ $\{0\}$, and $\operatorname{opt}\left(\mathcal{Y}\left(s_{k}\right) \mid h_{k-1} s_{k}\right)=\left\{X\left(s_{k}\right)+\epsilon\right\}$. However,

$$
\begin{aligned}
\operatorname{opt}\left(\mathcal{X}+\bigoplus_{s_{k}} E_{s_{k}} \mathcal{Y}\left(s_{k}\right) \mid h_{k-1}\right) & =\operatorname{opt}\left(\left\{X+\epsilon I_{E}: E \subseteq S_{k}\right\} \mid h_{k-1}\right) \\
& =\left\{X+\epsilon I_{E}: E \subseteq S_{k}\right\}
\end{aligned}
$$

because

$$
\underline{P}\left(X+\epsilon I_{E} \mid h_{k}\right) \leq \underline{P}\left(X+\epsilon \mid h_{k}\right)<\bar{P}\left(X \mid h_{k}\right)
$$

for all $E \subseteq S_{k}$. We have shown that locality fails, or, in other words, local linearity for $1 \leq k<n$ is necessary for locality to hold for interval dominance (we will establish local linearity for $k=n$ further).

Next, suppose that local linearity holds for $1 \leq k<n$, but

$$
\underline{P}\left(Z \mid h_{k-1}\right)>\underline{P}\left(\underline{P}\left(Z \mid h_{k-1} S_{k}\right) \mid h_{k-1}\right) .
$$

for some gamble $Z$ on $S_{k} \times \cdots \times S_{n}$ and $h_{k-1}$ (indeed, if equality does not hold, we must have a strict inequality as given, by [24, 6.3.5(5), p. 296]).

Consider the following sets of gambles:

$$
\begin{aligned}
\mathcal{X} & =\{0\} \\
\mathcal{Y}\left(s_{k}\right) & =\left\{Z\left(s_{k}, \cdot\right), \underline{P}\left(Z \mid h_{k-1} s_{k}\right)\right\} \text { for all } s_{k}
\end{aligned}
$$

Let $X=0$ and $Y\left(s_{k}\right)=\underline{P}\left(Z \mid h_{k-1} s_{k}\right)$. These gambles are maximal in their respective sets so they must also be optimal with respect to interval dominance. We have

$$
\bar{P}\left(X+\bigoplus_{s_{k}} E_{s_{k}} Y\left(s_{k}\right) \mid h_{k-1}\right)=\bar{P}\left(\underline{P}\left(Z \mid h_{k-1} S_{k}\right) \mid h_{k-1}\right)
$$

and by local linearity

$$
=\underline{P}\left(\underline{P}\left(Z \mid h_{k-1} S_{k}\right) \mid h_{k-1}\right)
$$

Now consider the gamble

$$
X+\bigoplus_{s_{k}} E_{k} Z\left(s_{k}, \cdot\right)=Z .
$$

We have

$$
\begin{aligned}
\underline{P}\left(Z \mid h_{k-1}\right) & >\underline{P}\left(\underline{P}\left(Z \mid h_{k-1} S_{k}\right) \mid h_{k-1}\right) \\
& =\bar{P}\left(X+\bigoplus_{s_{k}} E_{s_{k}} Y\left(s_{k}\right) \mid h_{k-1}\right),
\end{aligned}
$$


so we have found a gamble in $\mathcal{X}+\bigoplus_{s_{k}} E_{s_{k}} \mathcal{Y}\left(s_{k}\right)$ that dominates $X+\bigoplus_{s_{k}} E_{s_{k}} Y\left(s_{k}\right)$ with respect to interval dominance, and so locality fails. In other words, if local linearity holds for $1 \leq k<n$ then marginal extension is necessary for locality to hold for interval dominance. From our result earlier in the proof, this implies that local linearity for $1 \leq k<n$ and marginal extension are necessary.

Next, suppose that local linearity for $1 \leq k<n$ and marginal extension hold, but local linearty for $k=n$ fails. Then, there must be an $h_{n-1}=$ $h_{n-2} s_{n-1}^{\prime} \in H_{n-1}$, a gamble $Z$ on $S_{n}$, and an $\epsilon>0$, such that $\underline{P}\left(Z \mid h_{n-1}\right)+\epsilon<$ $\bar{P}\left(Z \mid h_{n-1}\right)$. Let $\mathcal{Y}\left(s_{n-1}\right)=\{0\}$ for all $s_{n-1}$ except $\mathcal{Y}\left(s_{n-1}^{\prime}\right)=\{Z\}$. Let $\mathcal{X}=\left\{0, \epsilon P\left(E_{s_{n-1}^{\prime}} \mid h_{n-2}\right)\right\}$.

Then by marginal extension and local linearity for stage $n-1$,

$$
\begin{aligned}
\bar{P}\left(0+E_{s_{n-1}^{\prime}} Z \mid h_{n-2}\right) & =P\left(E_{s_{n-1}^{\prime}} \bar{P}\left(Z \mid h_{n-1}\right) \mid h_{n-2}\right) \\
& =P\left(E_{s_{n-1}^{\prime}} \mid h_{n-2}\right) \bar{P}\left(Z \mid h_{n-1}\right) \\
& \geq P\left(E_{s_{n-1}^{\prime}} \mid h_{n-2}\right)\left(\epsilon+\underline{P}\left(Z \mid h_{n-1}\right)\right),
\end{aligned}
$$

but

$$
\begin{aligned}
\underline{P}\left(\epsilon P\left(E_{s_{n-1}^{\prime}} \mid h_{n-2}\right)+E_{s_{n-1}^{\prime}} Z \mid h_{n-2}\right) & =P\left(\epsilon P\left(E_{s_{n-1}^{\prime}} \mid h_{n-2}\right)+E_{s_{n-1}^{\prime}} \underline{P}\left(Z \mid h_{n-1}\right) \mid h_{n-2}\right) \\
& =P\left(E_{s_{n-1}^{\prime}} \mid h_{n-2}\right)\left(\epsilon+\underline{P}\left(Z \mid h_{n-1}\right)\right) .
\end{aligned}
$$

Therefore the gamble $0+E_{s_{n-1}^{\prime}} Z$ is optimal with respect to interval dominance. But clearly, opt $\left(\mathcal{X} \mid h_{n-2}\right)=\left\{\epsilon P\left(E_{s_{n-1}^{\prime}} \mid h_{n-2}\right)\right\}$ and so locality fails. In other words, if local linearity holds for $1 \leq k<n$ and marginal extension holds, then local linearity for $k=n$ is necessary for locality to hold for interval dominance.

So, marginal extension and local linearity for $1 \leq k \leq n$ are necessary for locality. The proof of Corollary 11 shows that these conditions imply full linearity of $\underline{P}$. In such a case, interval dominance and maximality coincide at every stage, and so by Proposition 6 , it is also necessary that $\underline{P}\left(E_{s_{k}} \mid h_{k-1}\right)>0$ for all $1 \leq k<n, s_{k} \in S_{k}$, and $h_{k-1} \in H_{k-1}$.

Interval dominance requires even stronger conditions than $\Gamma$-maximin. Indeed, we have the following corollary.

Corollary 11. If interval dominance satisfies locality on $S_{0}, \ldots, S_{n}$, then, for all $1 \leq k \leq n$, all gambles $X$ on $F_{k}$, and all $h_{k-1} \in H_{k-1}$,

$$
\underline{P}\left(X \mid h_{k-1}\right)=\bar{P}\left(X \mid h_{k-1}\right) .
$$

Proof. Almost identical to the proof of Corollary 9.

So, for interval dominance to satisfy locality, $\underline{P}$ must essentially correspond to a coherent prevision $E_{p}$ for some full conditional probability $p$ : you cannot be imprecise, and at the same time endorse locality for interval dominance. 


\section{Conclusion}

We identified a reasonable and convenient condition that solutions to a particular class of sequential decision problems ought to satisfy under act-state independence: locality. Essentially, locality means that, under fairly general circumstances, solving a sequential decision problem can be reduced to solving a sequence of static decision problems, each contingent on the state history of the process. Locality seems quite compelling for both practical and philosophical reasons. For example, any sequence of unrelated (in the sense of rewards and information) decision problems clearly ought to be solvable by solving each problem in the sequence independently of the others.

For those theories that can be modelled via choice functions on gambles (which includes many), we identified a necessary and sufficient condition for locality to be satisfied: sequential distributivity. This property provides a test for any decision criterion whether locality is satisfied or not. We applied it to a number of well known choice functions induced by coherent lower previsions: maximality, E-admissibility, $\Gamma$-maximin, and interval dominance.

For maximality and E-admissibility, the conditions for locality interestingly coincide, and amount to (i) having strictly positive lower transition probabilities, (ii) satisfying marginal extension. The first condition can be relaxed, if we are willing to adopt an (arbitrarily close) approximate version of maximality. The second condition, marginal extension, is satisfied if lower previsions are specified locally at each stage, conditional on the full history at that stage. This is the natural way of specifying a joint model for a sequential problem, hence, usually, marginal extension will be satisfied. Interestingly, we can also turn the argument around, and interpret locality as a behavioural motivation for marginal extension: you must satisfy marginal extension if you wish to satisfy locality.

For $\Gamma$-maximin, locality will usually be violated, unless a precise conditional prevision is specified, in which case it reduces to maximizing expected utility. However, local $\Gamma$-maximin solutions will still be globally maximal (i.e. undominated), hence for this reason, $\Gamma$-maximin is perhaps still not that unreasonable.

For interval dominance, locality will also usually be violated unless a precise conditional prevision is specified.

\section{References}

[1] Eitan Altman. Applications of Markov decision processes in communication networks: a survey. Technical report, INRIA, 2000.

[2] Yakov Ben-Haim. Info-Gap Decision Theory: Decisions Under Severe Uncertainty. Academic Press, 2001.

[3] G. De Cooman and M. C. M. Troffaes. Dynamic programming for deterministic discrete-time systems with uncertain gain. International Journal of Approximate Reasoning, 39(2-3):257-278, Jun 2005. 
[4] Thomas Dean, Leslie Pack Kaelbling, Jak Kirman, and Ann Nicholson. Planning under time constraints in stochastic domains. Artificial Intelligence, 76:35-74, 1995.

[5] A. P. Dempster. Upper and lower probabilities induced by a multivalued mapping. Ann. Math. Statist., 38:325-339, 1967.

[6] Lester E. Dubins. Finitely additive conditional probabilities, conglomerability and disintegrations. The Annals of Probability, 3(1):89-99, Feb 1975.

[7] T. Fabian, J. L. Fisher, M. W. Sasieni, and A. Yardeni. Purchasing raw material on a fluctuating market. Operations Research, 7(1):107-122, Jan.Feb. 1959

[8] Dave Ferguson and Anthony Stentz. Focussed processing of MDPs for path planning. In Proceedings of the 16th IEEE International Conference on Tools with Artificial Intelligence (ICTAI 2004), 2004.

[9] Gordon B. Hazen. Does rolling back decision trees really require the independence axiom? Management Science, 33(6):807-809, June 1987.

[10] Nathan Huntley and Matthias C. M. Troffaes. Subtree perfectness, backward induction, and normal-extensive form equivalence for single agent sequential decision making under arbitrary choice functions. In preparation.

[11] Archana Jayakumar and Sohrab Asgarpoor. Maintenance optimization of equipment by linear programming. Probability in the Engineering and Informational Sciences, 20:186-193, 2006.

[12] Peter Kolesar. A Markovian model for hospital admission scheduling. Management Science, 16(6):B384-B396, February 1970.

[13] A. N. Kolmogorov. Foundations of the Theory of Probability. Chelsea Publishing Company, New York, 1950.

[14] Irving H. LaValle and Kenneth R. Wapman. Rolling back decision trees requires the independence axiom! Management Science, 32(3):382-385, March 1986.

[15] Enrique Miranda. A survey of the theory of coherent lower previsions. International Journal of Approximate Reasoning, 48(2):628-658, 2008.

[16] John Rust. When is it optimal to kill off the market for used durable goods? Econometrica, 54(1):65-86, 1986.

[17] Leonard J. Savage. The Foundations of Statistics. Dover, New York, 1972. Second revised edition. 
[18] R. Selten. Reexamination of the perfectness concept for equilibrium points in extensive games. International Journal of Game Theory, 4(1):25-55, Mar 1975.

[19] Amartya Sen. Social choice theory: A re-examination. Econometrica, 45(1):53-89, January 1977.

[20] Glenn Shafer. A Mathematical Theory of Evidence. Princeton University Press, 1976.

[21] Glenn Shafer and Vladimir Vovk. Probability and Finance: It's Only a Game! Wiley, 2001.

[22] Richard S. Sutton and Andrew G. Barto. Reinforcement Learning: An Introduction. MIT Press, Cambridge, 1998. http://www.cs.ualberta.ca/ sutton/book/the-book.html.

[23] Matthias C. M. Troffaes. Finite approximations to coherent choice. International Journal of Approximate Reasoning, 50(4):655-665, April 2009.

[24] Peter Walley. Statistical Reasoning with Imprecise Probabilities. Number 42 in Monographs on Statistics and Applied Probability. Chapman and Hall, London, 1991.

[25] Peter Walley. Inferences from multinomial data: Learning about a bag of marbles. Journal of the Royal Statistical Society. Series B (Methodological), $58(1): 3-57,1996$.

[26] Peter M. Williams. Notes on conditional previsions. Technical report, School of Math. and Phys. Sci., Univ. of Sussex, 1975.

[27] K. Karen Yin, Hu Liu, and Neil E. Johnson. Markovian inventory policy with application to the paper industry. Computers and Chemical Engineering, 26:1399-1413, 2002. 
Apêndice III - Artigo: Aritificial

Intelligence 2011 


\title{
Sequential decision making with partially ordered preferences
}

\author{
Daniel Kikuti, Fabio Gagliardi Cozman*, Ricardo Shirota Filho \\ Escola Politécnica, Universidade de São Paulo, Av. Prof. Mello Moraes, 2231 São Paulo, SP, Brazil
}

\section{A R T I C L E I N F O}

\section{Article history:}

Received 28 February 2009

Received in revised form 11 August 2010

Accepted 11 August 2010

Available online 2 December 2010

\section{Keywords:}

Sequential decision making under uncertainty

Partially ordered preferences

Sets of probability measures

Criteria of choice

Consequentialist and resolute norms

Linear and multilinear programming

\begin{abstract}
A B S T R A C T
This paper presents new insights and novel algorithms for strategy selection in sequential decision making with partially ordered preferences; that is, where some strategies may be incomparable with respect to expected utility. We assume that incomparability amongst strategies is caused by indeterminacy/imprecision in probability values. We investigate six criteria for consequentialist strategy selection: $\Gamma$-Maximin, $\Gamma$-Maximax, $\Gamma$-Maximix, Interval Dominance, Maximality and E-admissibility. We focus on the popular decision tree and influence diagram representations. Algorithms resort to linear/multilinear programming; we describe implementation and experiments.
\end{abstract}

(c) 2010 Elsevier B.V. All rights reserved.

\section{Introduction}

It is often possible, in a decision problem, to express preferences that are completely ordered; that is, for every two alternatives, the decision maker either prefers one to the other, or is indifferent between them. In fact, expected utility theory is based on the assumption that revealed preferences are completely ordered. However, preferences are often partially ordered; examples can be found in the theory of CP-nets and the theory of nondeterministic planning, as briefly discussed in Section 2. When preferences are partially ordered, two alternatives may be incomparable and incomparability may fail to be transitive.

In this paper we focus on preferences that can be represented by a single utility function and a set of probability measures. Whenever there are incomplete or partial beliefs, or disagreements amongst experts concerning chances, one may fail to assign a precise probability value to every event, thus producing a partial order with respect to expected utility $[3,46,73]$. This is the situation we wish to focus on. Section 2 contains the necessary background on these topics.

The literature describes many criteria of choice when preferences are partially ordered [71]. These criteria are covered in Section 3 and can be roughly divided into two groups: (1) criteria that enforce a complete ordering amongst choices ( $\Gamma$-Maximin, $\Gamma$-Maximax and $\Gamma$-Maximix); and (2) criteria that select a set of incomparable actions (Interval Dominance, Maximality and E-admissibility). Practical approaches to decision making with sets of probabilities have been mainly limited to the first category; however, recent discussions [60] have highlighted theoretical and behavioral problems when using this group of criteria, and the second group of criteria has been advocated as a more adequate approach. Nevertheless, incomparability comes at a cost, and very little has been observed in the literature in terms of algorithmic progress, mainly due to computational complexity and inability to deal with incomparable choices.

\footnotetext{
This work has been financially supported by FAPESP, grants 2003/11165-9, 2004/09568-0, 2005/58090-9, 2008/03995-5.

* Corresponding author.

E-mail addresses: danielkikuti@yahoo.com.br (D. Kikuti), fgcozman@usp.br (F.G. Cozman), ricardo.shirota@poli.usp.br (R.S. Filho).
} 
There are also distinct behavioral norms when it comes to sequential decision making with partially ordered preferences; that is, whenever a sequence of decisions must be made. For instance, a decision maker may be resolute in that she commits herself to a complete strategy once and for all, or consequentialist in that she allows herself to change the current strategy in case another one is appropriate in view of the future possible choices.

The interplay between criteria of choice, behavioral norms, and models such as decision trees and influence diagrams has not been explored in the literature; this paper aims at filling this gap to some extent. There are indeed insights to be learned from an organized discussion of criteria of choice and behavioral norms; for instance, we discuss in Section 5 the fact that the standard LIMID model clashes with a consequentialist stance.

Another, more substantial, contribution of the paper is the development of algorithms for consequentialist sequential decision making expressed through decision trees [56] and influence diagrams [34]. Algorithms for decision making under $\Gamma$-Maximin and similar criteria have appeared in many settings [58,69,74], while algorithms for decision making under Maximality and E-admissibility have been suggested by Kyburg and Pittarelli [43] and proposed more recently by Kikuti et al. [42] and Utkin and Augustin [72]. ${ }^{1}$ Section 3 presents algorithms and computational analysis for several criteria of choice. The most valuable contribution of Section 3 is the algorithm for E-admissibility. We also present a new algorithm for strategy selection using linear programming in a family of decision trees where partial preferences have considerable regularity.

Sections 4 and 5 respectively present algorithms for decision making in problems specified through decision trees and influence diagrams. We should note the scarcity of previous literature on influence diagrams under partially ordered preferences, perhaps due to the fact that several criteria of choice require the manipulation of an exponential number of strategies. To reduce this complexity, we examine "ordered" LIMIDs, and we analyze both their conceptual foundation (in particular their clash with consequentialism) and their computational properties.

In short, we present novel results and algorithms for sequential decision making with decision trees and influence diagrams, plus new insights for single-stage decision making under Interval Dominance and E-admissibility. The broader goal of the paper is to combine both the philosophical underpinnings and the computational properties of partially ordered preferences, a combination we feel is missing in the current literature.

\section{Partially ordered preferences, behavioral norms, and credal sets}

Throughout, our decision makers must select one or more actions within a finite set of possible alternatives $\mathbb{A}=$ $\left\{a_{1}, \ldots, a_{m}\right\}$. Performing action $a$ yields a reward $a(\omega)$ for each state of nature $\omega$; the set of states of nature is assumed to be a finite set $\Omega=\left\{\omega_{1}, \ldots, \omega_{n}\right\}$. We assume that $a(\omega)$ is a real number expressed in utiles. Even though some theories of preference allow multiple utilities to be defined for a single decision problem [2], in this paper we assume that utilities are precisely fixed in a given decision problem, and consequently every action is identified with a single real-valued function over the states of nature. Note that a utility function is a function that returns a value in utiles for each possible outcome; so we are assuming that a single utility function is fixed.

The connection between preference and expected utility, in decision making under risk [47], is based on the axiomatization of preference relations. Denote the strict preference of $a_{i}$ over $a_{j}$ by $a_{i} \succ a_{j}$, and define indifference between two actions as $a_{i} \sim a_{j} \Leftrightarrow \neg\left(a_{i} \succ a_{j}\right) \wedge \neg\left(a_{j} \succ a_{i}\right)$. Suppose $\succ$ satisfies (recall that actions are functions that can be multiplied and added) [23]:

Axiom 1 (completeness). The relation $\succ$ is complete and negatively transitive (recall that $\succ$ is negatively transitive if it satisfies for all $\left.a_{i}, a_{j}, a_{k}:\left(a_{i} \nsucc a_{j}\right) \wedge\left(a_{j} \nsucc a_{k}\right) \Rightarrow\left(a_{i} \nsucc a_{k}\right)\right)$.

Axiom 2 (independence). For $\alpha \in(0,1], a_{i} \succ a_{j} \Rightarrow \alpha a_{i}+(1-\alpha) a_{k} \succ \alpha a_{j}+(1-\alpha) a_{k}$ (this axiom says that whenever $a_{i} \succ a_{j}$, a compound action made of $a_{i}$ and $a_{k}$ will be preferred to a compound action made of $a_{j}$ and $a_{k}$, where $\alpha$ denotes the ratio of mixture between the actions).

Axiom 3 (continuity). If $a_{i} \succ a_{j} \succ a_{k}$, then there exists $\alpha, \beta \in(0,1)$ such that $\alpha a_{i}+(1-\alpha) a_{k} \succ a_{j} \succ \beta a_{i}+(1-\beta) a_{k}$.

Then there must exist a single probability measure $P$ and a related expected utility representation for $>$; that is, the value of an action $a_{i}$ is given by $E\left[a_{i}\right]=\sum_{j=1}^{n} P\left(\omega_{j}\right) a_{i}\left(\omega_{j}\right)$, and $a_{i} \succ a_{j}$ if and only if $E\left[a_{i}\right]>E\left[a_{j}\right]$.

Several theories relax these axioms, attempting to accommodate various observed decision making patterns [1,20,40]. For instance, lexicographic preferences violate Axiom 3 and are encoded through expected utility vectors, ordered with respect to a lexicographic hierarchy [5,23]. Other theories violate Axiom 2 and lead to non-additive functionals that represent preferences [49, Section 2.3]. Partially ordered preferences violate Axiom 1 by assuming that preferences are not completely ordered; this is exactly the situation we examine in the present paper. If we assume a single utility function and do not require the preference relation $\succ$ to be complete, we have that $a_{i} \succ a_{j}$ if and only if $E_{P}\left[a_{i}\right]>E_{P}\left[a_{j}\right]$ for all $P$ in

\footnotetext{
1 Most results are based on material presented in Kikuti et al. [42] and Kikuti and Cozman [41]. The third author participated in developing the column generation method, and the experiments, reported in Section 4.3 .
} 


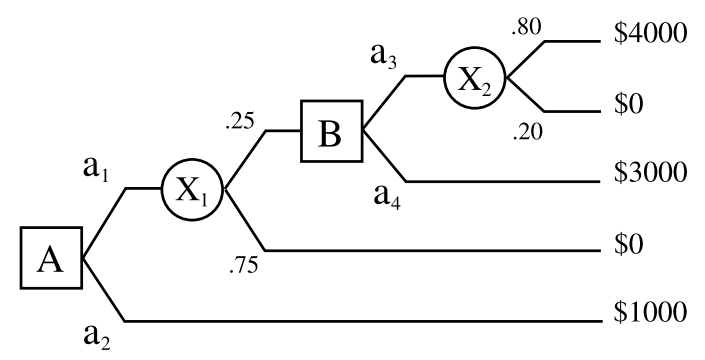

Fig. 1. A sequential decision problem represented through a decision tree.

a set of probability measures $[26,61,62]$. That is, the preference relation $\succ$ can be completely represented by a set of probability measures $K$. Incomparability between two actions $a_{i}$ and $a_{j}$ appears when one probability measure $P_{1} \in K$ produces $E_{P_{1}}\left[a_{i}\right]>E_{P_{1}}\left[a_{j}\right]$ while another probability measure $P_{2} \in K$ fails to produce $E_{P_{2}}\left[a_{i}\right]>E_{P_{2}}\left[a_{j}\right]$.

As alluded to in Section 1, several circumstances may preclude the assessment of a complete preference ordering, from incomplete understanding of a decision scenario, or perhaps a desire to abstract elements of a complex decision situation, to disagreements amongst experts involved in decision making. Sometimes the very language in which preferences are expressed allows for partial specification; this is particularly relevant in artificial intelligence applications. For instance, the semantics of "nondeterministic" actions in planning [6] is that these actions have effects whose probabilities are unknown, and consequently it is not possible to completely order them with respect to expected utility [69]. Another suggestive example is the theory of CP-nets [7], in which a graph-theoretical language organizes preferences about features of outcomes rather than outcomes themselves. A CP-net may generate a single preference ordering for outcomes, but in general it specifies a partial ordering. While a CP-net deals with outcomes (and thus reflects incomplete specification of utilities), a similar language for actions would be within the confines of the present paper. Hopefully the present paper will help shorten the gap between the current theories of nondeterministic planning and CP-nets, and the foundational literature on partially ordered planning.

When preferences are partially ordered, there may be no single "best" action to select. Before we examine criteria of choice in Section 3, we review behavioral norms for sequential decision problems (Section 2.1) and some properties of sets of probability measures (Section 2.2).

\subsection{Sequential decision problems: strategies and behavior norms}

In a sequential decision problem, a decision maker faces a sequence of decisions, and each decision may impact future decisions. A convenient language to introduce sequential decision problems is through decision trees [56]. A decision tree $\mathcal{T}$ is a connected graph without cycles, where each node belongs to one of three categories. A decision node $D \in \mathbb{D}$, typically drawn as a square, represents the place where the decision maker must choose an action. A chance node $C \in \mathbb{C}$, typically drawn as a circle, represents an event out of control of the decision maker. A utility node $U \in \mathbb{U}$ is associated with a realvalued utility. In decision trees, a leaf node is a utility node and vice versa. Edges out of a decision node represent the possible actions that the decision maker can choose and edges out of a chance node represent the possible outcomes of the event. A subtree of $\mathcal{T}$ is a tree $\mathcal{T}^{\prime}$ whose nodes and edges form subsets of $\mathcal{T}$. We assume that any tree or subtree is rooted at a decision node. A strategy is a complete set of actions specifying how the decision maker should act when she is actually called to decide. A strategy for a subtree is called a substrategy. We are interested in selecting strategies. The next example clarifies this notion.

Example 1. The decision tree in Fig. 1 is adapted from [40]. It has three strategies: $s_{1}=\left(a_{1}, a_{3}\right), s_{2}=\left(a_{1}, a_{4}\right)$ and $s_{3}=\left(a_{2}\right)$; where $\left(a_{1}, a_{3}\right)$ means that the decision maker will choose action $a_{1}$ at $A$ and $a_{3}$ if she reaches the decision node $B$ (if she does not reach $B$ then she receives $\$ 0$ ). (One might instead consider all conceivable combinations of decisions, as often done in game theory; in this case we would have strategies such as $\left(a_{2}, a_{3}\right)$ and $\left(a_{2}, a_{4}\right)$. We do not follow this route.)

There are two widely debated behavioral norms for decision makers engaged in sequential decision problems [21]. A resolute decision maker commits herself to a complete strategy once and for all, comparing simultaneously all strategies rooted at the first decision node [50]. A sophisticated or consequentialist decision maker selects strategies to follow out of a decision node only looking at the subtree rooted at that decision node, and may actually change the strategy previously selected $[28,29]$. There are other possible norms that we do not investigate further; for instance, a myopic decision maker constructs her strategy by selecting actions, at each decision node, independently of future choices $[28,67]$.

The following example illustrates the behavioral norms in the light of a non-expected utility model of preference.

Example 2. Consider again Example 1. Suppose the decision maker has adopted a rank-dependent utility model of preference [40], where strategies are ranked using a single utility/probability pair and the function 


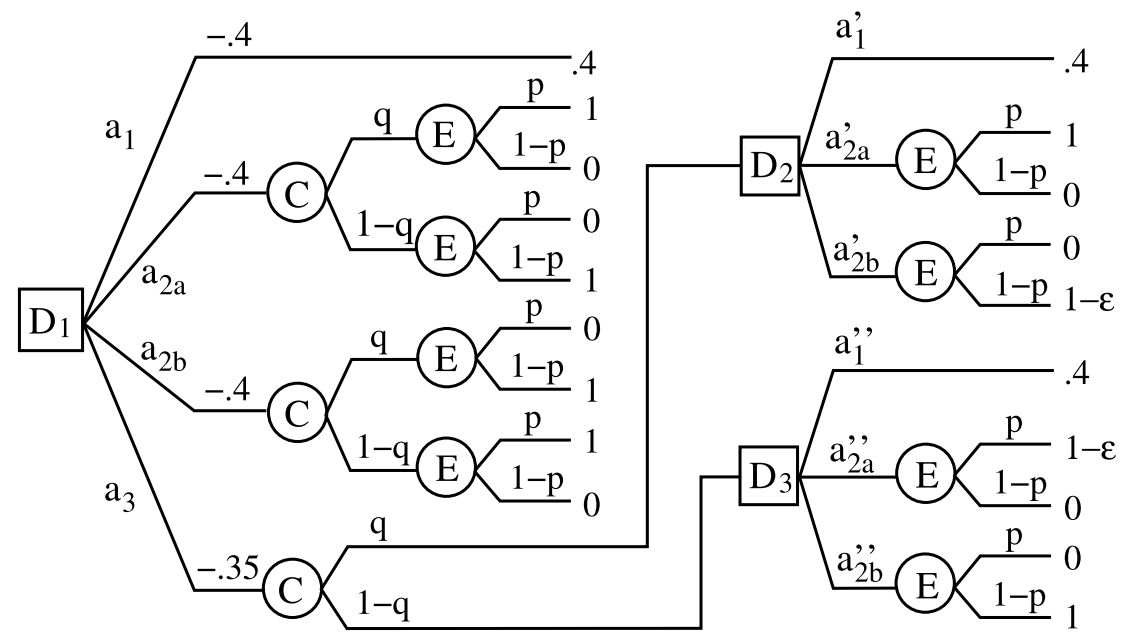

Fig. 2. Decision tree for Example 3.

$$
V(a)=a\left(\omega_{1}\right)+\sum_{j=2}^{n} \exp \left(-\left(-\ln \left(x_{j}\right)\right)^{0.5}\right)\left[a\left(\omega_{j}\right)-a\left(\omega_{j-1}\right)\right],
$$

where $x_{j}=\sum_{k=j}^{n} P\left(\omega_{k}\right)$ and the inequalities $a\left(\omega_{1}\right) \leqslant \cdots \leqslant a\left(\omega_{n}\right)$ are assumed to hold. At node $A$ we have $V\left(s_{1}\right)=1124.86$, $V\left(s_{2}\right)=924.23$ and $V\left(s_{3}\right)=1000$, so at node $A, s_{1} \succ s_{3} \succ s_{2}$. However at node $B$, action $a_{4}$ is preferred to $a_{3}$ as $V\left(a_{3}\right)=$ 2494.06 and $V\left(a_{4}\right)=3000$. If the decision maker is resolute, she selects $s_{1}$ and implements it; at $B$ she must choose $a_{3}$ even if $a_{4}$ is locally better. If the decision maker is consequentialist, she would anticipate that in $B$ she would prefer $a_{4}$ to $a_{3}$, so $s_{1}$ is infeasible for her: comparing $s_{2}$ and $s_{3}$ at $A$, she must choose $s_{3}$. If the decision maker is myopic, she would select $s_{1}$ at $A$, but when she reaches $B$ she deviates from $s_{1}$ by choosing $a_{4}$ (the best option locally), thus actually implementing $s_{2}$.

In general, resolute behavior demands examination of all strategies at the beginning. Even though the resolute norm has been forcefully defended when moral aspects of commitments are taken into account [9,51], resolution faces several problems as to how preferences are to be elicited and modeled. By renouncing consequentialism, the decision maker does not have well defined local preferences that can be revealed from her choices [57]. ${ }^{2}$ A consequentialist norm makes even more sense if we consider a resource bounded decision maker who cannot possibly optimize over the space of all strategies [66]. For this reason, we adopt the consequentialist norm throughout this paper.

If a decision maker eliminates an action at a non-root node, and this action might be selected from the perspective of the root node, we have an episode of incoherent choice. Another situation is that of inconsistent choice, where the decision maker selects a strategy but subsequently deviates from it $[28,49]$. Incoherent and inconsistent choices do not occur when a decision maker ranks preferences through expected utility with a single utility function and a single non-zero probability measure (in fact, resolute and consequentialist norms are equivalent for such a decision maker). However, incoherence may befall the decision maker when preferences are partially ordered:

Example 3 (Adapted from Seidenfeld [60]). In the decision tree depicted in Fig. 2, $p \in[0.25,0.75], q=1 / 2$ and $\epsilon>0$. There is a charge of 0.4 utiles to take action $a_{1}, a_{2 a}$ or $a_{2 b}$, and a charge of 0.35 utiles to take action $a_{3}$, thus $E_{P}\left[a_{1}\right]=0$ and $E_{P}\left[a_{2 a}\right]=E_{P}\left[a_{2 b}\right]=0.1$ for every possible $P$. There are 9 additional strategies to consider, by combining actions out of $D_{2}$ and $D_{3}$. Suppose we are interested in strategies with maximum minimum expected utility (that is, we adopt the $\Gamma$-Maximin criterion to be detailed later). For small $\epsilon$, at $D_{2}$ the $\Gamma$-Maximin action is $a_{1}^{\prime}$, and at $D_{3}$ the $\Gamma$-Maximin action is $a_{1}^{\prime \prime}$; however the strategy $\left(a_{3}, a_{1}^{\prime}, a_{1}^{\prime \prime}\right)$ is not $\Gamma$-Maximin at $D_{1}$, as $\left(a_{3}, a_{2 a}^{\prime}, a_{2 b}^{\prime \prime}\right)$ is a strategy with larger minimum expected utility. This is an episode of incoherent choice, since we throw away the actions that would lead to better strategy at the root node.

We will further discuss incoherent and inconsistent choices in Section 4.1, after detailing some criteria of choice.

\subsection{Partially ordered preferences through sets of probability measures}

As noted previously, partially ordered preferences can often be represented by sets of probability measures. We call a set of probability measures a credal set [46], and denote by $K(X)$ a credal set that contains distributions for a random

\footnotetext{
2 Jaffray [38] combines consequentialist preferences and non-consequentialist behavior. Nielsen and Jaffray [52] use the rank-dependent utility model with preferences function revealed by the anticipated utility theory of Quiggin [55].
} 
variable $X$. We assume throughout that credal sets are given by a finite set of linear constraints, thus being closed convex with finitely many vertices (Example 4 shows one such credal set).

Given a set of assessments containing constraints on probability values, any credal set that satisfies the constraints is an extension of the assessments. Given an event $A, \underline{P}(A)=\min _{P \in K} P(A)$ and $\bar{P}(A)=\max _{P \in K} P(A)$ are respectively the lower probability and the upper probability of $A$. Given a random variable $X, \underline{E}[X]=\min _{P \in K} E[X]$ and $\bar{E}[X]=\max _{P \in K} E[X]$ are respectively the lower expectation and the upper expectation of $X$ (we use expected value and expectation as synonyms). A conditional credal set $K(\cdot \mid A)$ is obtained by conditioning (Bayes' rule) every measure in a credal set with respect to $A$ (likewise, $K(\cdot \mid Y)$ is produced by elementwise conditioning with respect to a random variable $Y$ ). We assume that any conditioning event has lower probability strictly larger than zero $(\underline{P}(A)>0)$. If the (joint) credal set $K(X, Y)$ is such that every one of its vertices satisfies stochastic independence of $X$ and $Y$ (that is, all vertices factorize as $P(X) P(Y)$ ), then $X$ and $Y$ are said to be strongly independent. There are several other concepts of independence for credal sets in the literature [11,12]; strong independence is perhaps the most popular, and we adopt it in this paper. Conditional strong independence is defined in the obvious manner, by requiring conditional stochastic independence for every vertex of the conditional credal set of interest.

We shall, when we deal with influence diagrams, use elements of the theory of credal networks. A credal network is a graph-theoretical representation for a joint credal set $K\left(X_{1}, \ldots, X_{n}\right)$ that mimics the structure of a Bayesian network [13]. A credal network consists of a directed acyclic graph such that each node is identified with a random variable $X_{i}$. The parents of variable $X_{i}$ in the graph are denoted by $\mathrm{pa}\left(X_{i}\right)$. Each variable is associated with a conditional credal set $K\left(X_{i} \mid \mathrm{pa}\left(X_{i}\right)=\pi_{k}\right)$ for each value $\pi_{k}$ of $\mathrm{pa}\left(X_{i}\right)$, and every variable is assumed strongly independent of its nondescendants in the graph given its parents in the graph. Thus the largest extension of all assessments in a credal network, called the strong extension, is a joint credal set $K\left(X_{1}, \ldots, X_{n}\right)$ given by the convex hull of the set of joint distributions: $\left\{\prod_{i=1}^{n} p\left(X_{i} \mid \operatorname{pa}\left(X_{i}\right)\right): p\left(X_{i} \mid \mathrm{pa}\left(X_{i}\right)=\pi_{k}\right) \in K\left(X_{i} \mid \mathrm{pa}\left(X_{i}\right)=\pi_{k}\right)\right\}$ where this expression refers to densities induced by the appropriate probability distributions. An inference is then the computation of lower/upper probabilities for the values of some variable. In general, inference with strong extensions is $N P^{P P}$-complete; with constraints on the induced width of credal networks, the complexity of inferences is in $N P$ [17]. The best available algorithms for inference with strong extensions optimize a multilinear polynomial $\prod_{i=1}^{n} p\left(X_{i} \mid \mathrm{pa}\left(X_{i}\right)\right)$ subject to assessments in the credal network [16]. Some variables may be discarded when computing a particular inference, using the $d$-separation property that strong extensions inherit from Bayesian networks [12]. ${ }^{3}$

We will need to solve multilinear programs several times in this paper. The most refined algorithm for solution of multilinear programming problems arising from judgements of independence seems to be the adaptation of Sherali and Tuncbilek [65]'s RL method by de Campos and Cozman [17]. We have used the adapted RL method in our implementation (Section 5.2), and we usually take the solution of a multilinear program to be a "unit" of computation, even though such a solution may require substantial effort in itself.

\section{Criteria of choice in single-stage decision making}

In this section we study several criteria of choice for partially ordered preferences; that is, criteria that select one or more actions from a given set of actions. We present the basic computations that must be performed in a single-stage decision making problem, and we present short code fragments that are used later. While several of these algorithms have appeared in the literature [70,71], the discussion contributes with new analyses both for Interval Dominance and for E-admissibility [42]. The computational cost of the algorithms is presented as a function of the number of auxiliary optimization programs that must be solved. The following example clarifies the nature of these programs (the example deals with linear constraints; later we face situations where auxiliary programs are multilinear).

Example 4. Consider a decision problem with three states, $x_{1}, x_{2}$ and $x_{3}$ that are values of a random variable $X$. Actions and utilities are given in Fig. 3. Suppose the credal set $K(X)$ is specified through $P\left(X=x_{1}\right) \in[1 / 10 ; 7 / 20], P\left(X=x_{2}\right) \in$ $[1 / 5 ; 2 / 5]$, and $P\left(X=x_{3}\right) \in[7 / 20 ; 13 / 20]$, as depicted in Fig. 3. To obtain lower and upper expectations for actions $a_{i}$ (also in Fig. 3), we must solve linear programs of the form $\min / \max E\left[a_{i}\right]=\sum_{j} p_{j} a_{i}\left(x_{j}\right)$ subject to $\sum_{j} p_{j}=1$ and $\underline{P}\left(X=x_{j}\right) \leqslant$ $p_{j} \leqslant \bar{P}\left(X=x_{j}\right)$ for $j=1,2,3$.

Existing criteria can be roughly divided into two groups. Indecision-resistant criteria force a single ordering of choices, and thus select either a single action or a set of equally ranked (with respect to choice) actions. Indecision-prone criteria may return a set of actions that are deemed incomparable (with respect to preference).

We start by briefly examining indecision-resistant criteria: $\Gamma$-Maximin, $\Gamma$-Maximax, and $\Gamma$-Maximix. The $\Gamma$-Maximin criterion selects an action with highest lower expectation, a "pessimistic" solution that focuses on worst case scenarios [3,25]. Algorithm 1 is an easy translation of the $\Gamma$-Maximin criterion. The $\Gamma$-Maximax criterion selects an action with highest upper expectation, an "optimistic" solution that focuses on best case scenarios [58]. The $\Gamma$-Maximix criterion selects

\footnotetext{
3 Given three collections of variables $\mathbf{X}, \mathbf{Y}$ and $\mathbf{Z}$, suppose that along every path between a variable in $\mathbf{X}$ and a variable in $\mathbf{Y}$ there is a variable $W$ such that: either $W$ has two converging arrows and is not in $\mathbf{Z}$ and none of its descendants are in $\mathbf{Z}$, or $W$ is in $\mathbf{Z}$. Then $\mathbf{X}$ and $\mathbf{Y}$ are d-separated by $\mathbf{Z}$ [54].
} 


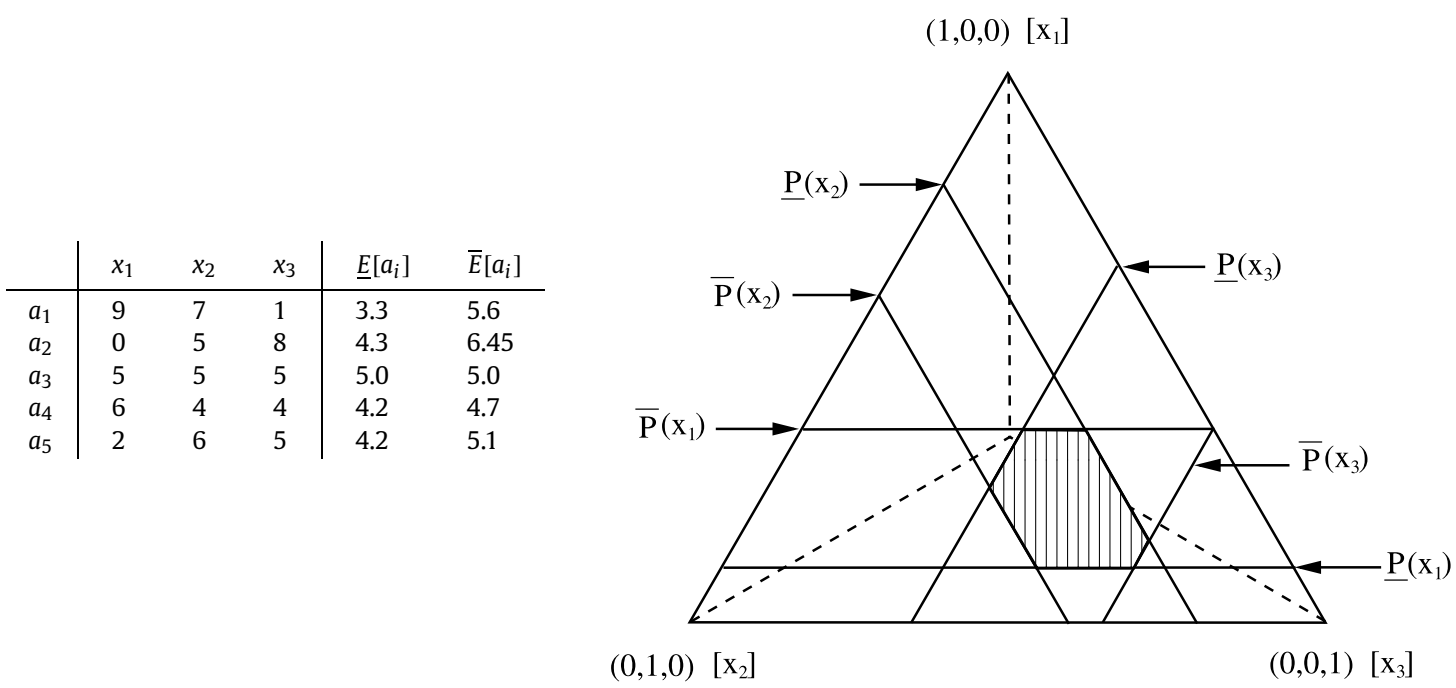

Fig. 3. Left: Actions, utilities, and lower and upper expected utilities for Example 4. Right: Credal set $K(X)$ (hatched area) defined by probability intervals in Example 4; axes denote probability of $x_{1}, x_{2}$ and $x_{3}$.
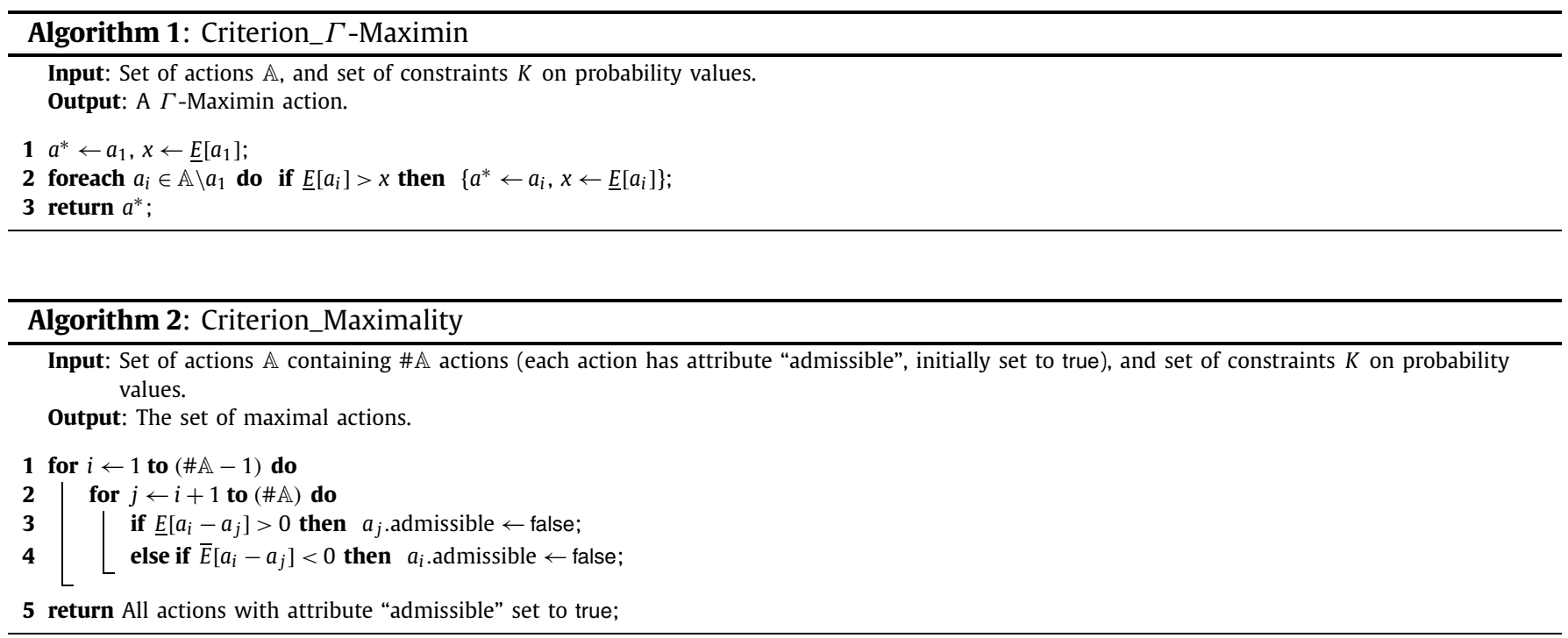

$a^{*}=\arg \max _{a_{i} \in \mathbb{A}}\left(\eta \underline{E}\left[a_{i}\right]+(1-\eta) \bar{E}\left[a_{i}\right]\right)$, where $\eta \in[0,1]$ reflects the degree of ambiguity aversion [72], and is already sketched by Hurwicz [36]. Algorithm 1 can be easily modified to deal with the $\Gamma$-Maximax and $\Gamma$-Maximix criteria. For these three criteria, Algorithm 1 returns the selected action by solving a number of optimization programs, each subject to constraints in $K$. The number of optimization programs is clearly linear on the number of actions. In Example 4 , the $\Gamma$-Maximin criterion selects $a_{3}$, while the $\Gamma$-Maximax criterion selects $a_{2}$ and the $\Gamma$-Maximix criterion also selects $a_{2}$ for $\eta=0.5$.

Consider indecision-prone criteria, starting with Maximality. An action $a_{i}$ is maximal if there is no action $a_{j}$ such that, for each possible probability measure $P \in K, E_{P}\left[a_{j}\right]>E_{P}\left[a_{i}\right]$. The maximality criterion is based on pairwise comparisons amongst actions, as indicated by Algorithm 2 (in Algorithm 2 we use the fact that $E_{P}\left[a_{i}\right]>E_{P}\left[a_{j}\right]$ for all $P$ is equivalent to $\underline{E}\left[a_{i}-a_{j}\right]>0$ [73]). To handle $n$ actions, the algorithm must solve at most $\left(n^{2}-n\right)$ optimization programs. In Example 4 , to determine whether $a_{4}$ can be maximal in the presence of $a_{3}$, we must solve the linear program $\max \left(6 p_{1}+4 p_{2}+4 p_{3}-\right.$ $\left.5 p_{1}-5 p_{2}-5 p_{3}\right)$ subject to $\sum_{i=1}^{3} p_{i}=1,1 / 10 \leqslant p_{1} \leqslant 7 / 20,1 / 5 \leqslant p_{2} \leqslant 2 / 5$, and $7 / 20 \leqslant p_{3} \leqslant 13 / 20$. We find that the maximum is $3 / 10$, hence $a_{4}$ is not maximal when $a_{3}$ is present.

We now examine Interval Dominance and E-admissibility in more detail.

\subsection{Interval Dominance}

Interval Dominance selects one or more I-admissible actions as follows [70]. Action $a_{j}$ is I-inadmissible when $a_{i}$ is present if $\underline{E}\left[a_{i}\right]>\bar{E}\left[a_{j}\right]$. The I-admissible actions are the actions that are never I-inadmissible. Note that Interval Dominance does not identify dominance in the sense that, given two actions $a_{i}$ and $a_{j}, a_{i}$ dominates $a_{j}$ if for all probability measures $P$, 

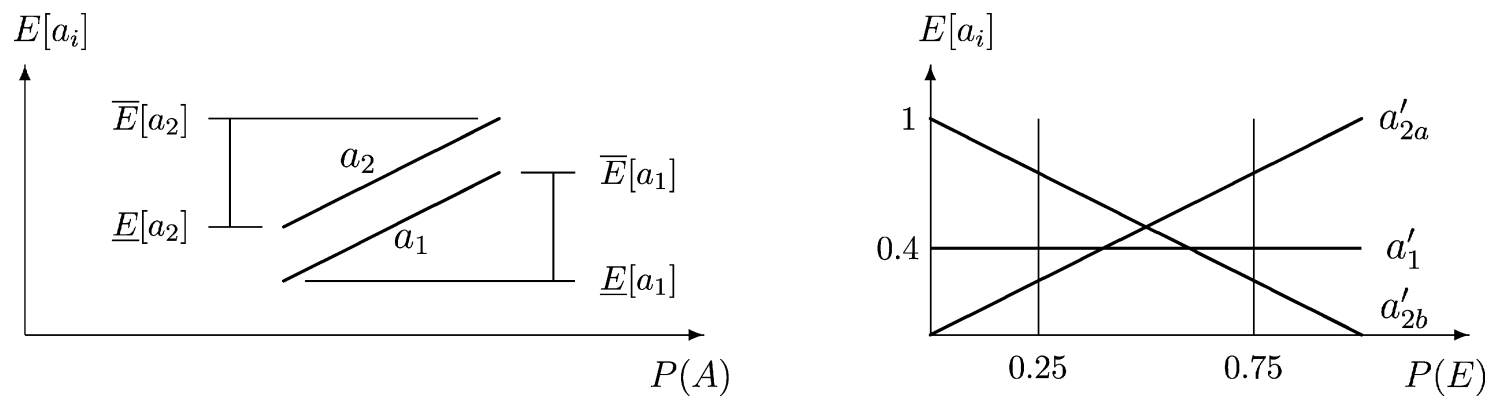

Fig. 4. Left: Interval Dominance does not capture "true" dominance (slanted lines denote the expectations of actions $a_{1}$ and $a_{2}$ as the probability of event $A$ varies). Right: Actions and their expected utilities in Example 5.

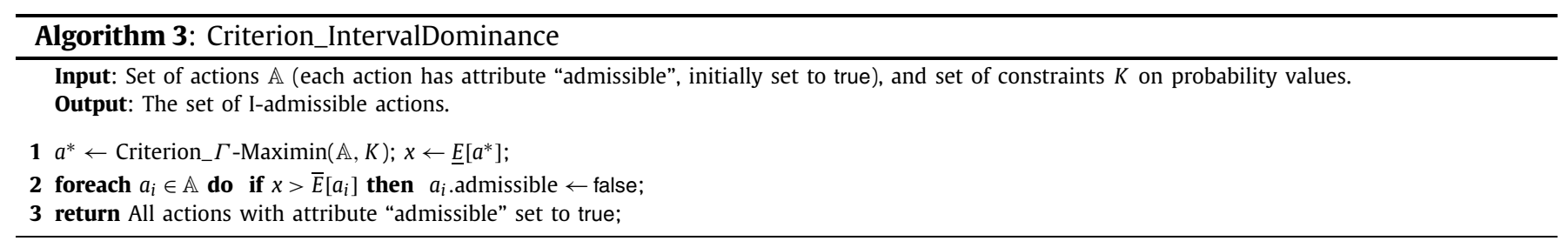

$E_{P}\left[a_{i}\right] \geqslant E_{P}\left[a_{j}\right]$ : in Fig. $4, a_{2}$ has higher expectation than action $a_{1}$ for every probability value $P(A) \in[0.3,0.7]$, but Interval Dominance does not choose between these actions.

A naive method to generate I-admissible actions would compare every pair of actions. Algorithm 3 avoids unnecessary computation of lower and upper expectations by using the $\Gamma$-Maximin solution $a^{*}$. Action $a^{*}$ is always I-admissible: suppose otherwise that $a^{*}$ is I-inadmissible; then there is $a^{\prime}$ with higher lower expectation, contradicting the hypothesis. The comparison of all actions with $a^{*}$ generates the I-admissible actions (an action $a^{\prime}$ dominated by another action $a^{\prime \prime}$ is also dominated by action $a^{*}$ with the maximum lower expectation, because $\underline{E}[a] \geqslant \underline{E}\left[a^{\prime \prime}\right]$ ). To find $a^{*}$ we must solve $n$ optimization programs; to determine the set of admissible actions we must solve $n-1$ additional optimization programs. Consequently, Algorithm 3 returns the I-admissible actions by solving a linear (on the number of actions) number of optimization programs.

\subsection{E-admissibility}

The criterion of E-admissibility, where E stands for "expectation" [45], focuses on actions that maximize expected utility. Given a set of actions $\mathbb{A}$ and a credal set $K$, the action $a_{i} \in \mathbb{A}$ is E-admissible when, for at least one $P \in K, a_{i}$ maximizes expected utility [59]:

$$
a_{i} \text { is E-admissible when } \exists(P \in K): \forall\left(a_{j} \in \mathbb{A}, j \neq i\right): E_{P}\left[a_{i}-a_{j}\right] \geqslant 0 \text {. }
$$

A variant of E-admissibility has been explored for Markov decision processes with imprecise probabilities by Itoh and Nakamura [37].

Example 5. Take $a_{1}^{\prime}, a_{2 a}^{\prime}$ and $a_{2 b}^{\prime}$ as in Example 3, and assume $\epsilon=0$. Expected utilities are shown in Fig. 4 (right). Only actions $a_{2 a}^{\prime}$ and $a_{2 b}^{\prime}$ are E-admissible: action $a_{2 b}^{\prime}$ maximizes expected utility for $P(E) \in[0.25,0.5]$, while action $a_{2 a}^{\prime}$ maximizes expected utility for $P(E) \in[0.5,0.75]$. Even though $a_{1}^{\prime}$ is the $\Gamma$-Maximin action, it never maximizes expected utility and is not E-admissible.

E-admissibility is qualitatively different from the previous criteria in that it does not depend on pairwise comparisons; rather, it is based on the existence of specific probability measures in the underlying credal set. Thus one might think that E-admissibility is more difficult to handle computationally than the other criteria. This feeling transpires in the literature on decision making with partially ordered preferences, as best expressed in Troffaes' [70] excellent review. However, it is possible to reduce the search for E-admissible actions to a linear sequence of optimization programs, using insights first derived by Kyburg and Pittarelli [43]. The original discussion by Kyburg and Pittarelli did not focus on computational cost, and it lay dormant until the same techniques surfaced independently in work by Kikuti et al. [42] and Utkin and Augustin [72], in response to Troffaes' [70] analysis.

The basic idea is that an action $a_{i}$ is E-admissible if there is $P \in K$ such that all constraints generated by Expression (1) are satisfied. If these constraints cannot be satisfied, then $a_{i}$ is not E-admissible. Algorithm 4 generates exactly these constraints: the linear expression $E_{P}\left[a_{i}-a_{j}\right] \geqslant 0$ in line 3 , stored in the set $\mathcal{C}$, denotes a symbolic constraint on the free (not yet bound) values of $P$. This way the algorithm avoids the need to represent credal sets explicitly (that is, the need to enumerate vertices). As every action is verified only once: 


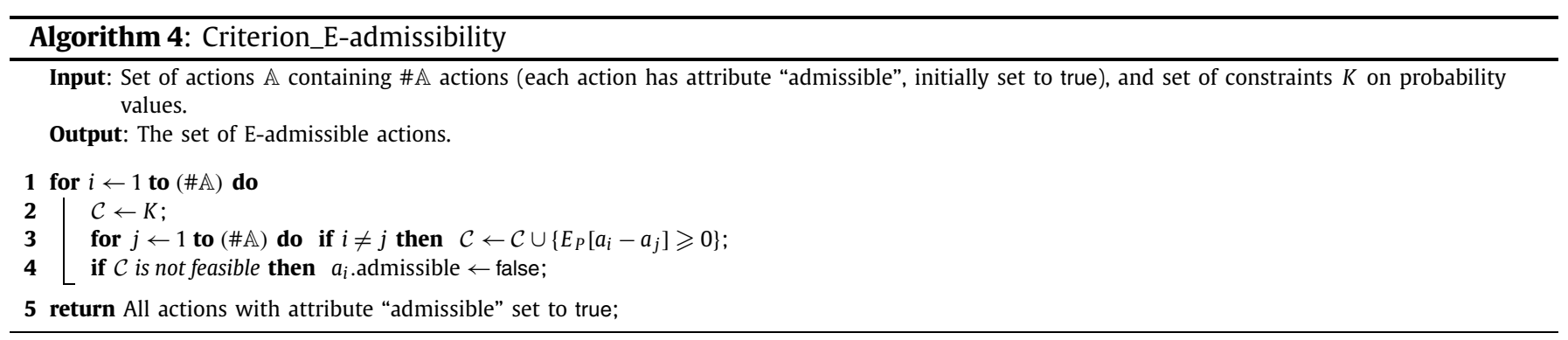

Proposition 1. Algorithm 4 returns the E-admissible actions by solving a linear (in the number of actions) number of optimization programs.

In Example 4, $a_{1}, a_{2}$ and $a_{3}$ are E-admissible actions. To verify whether $a_{1}$ is E-admissible, we must verify whether the following linear constraints can be satisfied together: $\sum_{i=1}^{3} p_{i}=1$ and

$$
\begin{aligned}
& 1 / 10 \leqslant p_{1} \leqslant 7 / 20, \quad 1 / 5 \leqslant p_{2} \leqslant 2 / 5, \quad 7 / 20 \leqslant p_{3} \leqslant 13 / 20, \\
& 9 p_{1}+7 p_{2}+p_{3}-0 p_{1}-5 p_{2}-8 p_{3} \geqslant 0, \quad 9 p_{1}+7 p_{2}+p_{3}-5 p_{1}-5 p_{2}-5 p_{3} \geqslant 0, \\
& 9 p_{1}+7 p_{2}+p_{3}-6 p_{1}-4 p_{2}-4 p_{3} \geqslant 0, \quad 9 p_{1}+7 p_{2}+p_{3}-2 p_{1}-6 p_{2}-5 p_{3} \geqslant 0 \text {. }
\end{aligned}
$$

\section{Algorithms for sequential decision making: decision trees}

In this section we derive algorithms for sequential decision making with decision trees that display indeterminacy/imprecision in probability values.

\subsection{Preliminaries: the option for consequentialism}

In a decision tree where chance nodes are associated with credal sets we may face differences between resolute and consequentialist behaviors.

We start by noting that E-admissibility and Maximality never lead to incoherent choice as both resolute and consequentialist norms produce identical sets of strategies [35,60]. ${ }^{4}$ Thus with E-admissibility and Maximality it is possible to run backward induction and produce a sequence of substrategies that satisfy consequentialism and that reach the strategies complying with the resolute norm. As a digression, note that such backward induction scheme is exactly given by Algorithm 5, detailed later, when this algorithm is specialized to the E-admissibility and Maximality criteria [42]; we also note that recent work by Huntley and Troffaes [35] yields simplifications to Algorithm 5 when applied to Maximality.

The remaining criteria in Section 3 may produce distinct consequentialist and resolute behaviors. The $\Gamma$-Maximin criterion was considered in Example 3. Clearly the same applies to the $\Gamma$-Maximix and $\Gamma$-Maximax criteria, since they do not guarantee that a discarded substrategy is not part of an optimal strategy. To illustrate this for $\Gamma$-Maximax criterion, consider again Example 3. At $D_{2}$, the $\Gamma$-Maximax action is $a_{2 a}^{\prime}$, and at $D_{3}$, the $\Gamma$-Maximax action is $a_{2 b}^{\prime \prime}$. However at $D_{1}$ the $\Gamma$-Maximax strategy is either $\left(a_{3}, a_{2 a}^{\prime}, a_{2 a}^{\prime \prime}\right)$ or $\left(a_{3}, a_{2 b}^{\prime}, a_{2 b}^{\prime \prime}\right)$.

Incoherent choice can also happen with Interval Dominance, a fact that apparently has not been indicated before:

Example 6. In the decision tree depicted in Fig. 5, suppose $p \in[1 / 10,3 / 10], q_{1} \in[1 / 5,2 / 5], q_{2} \in[2 / 5,3 / 5]$ and $q_{3} \in$ $[3 / 5,4 / 5]$. At $D_{2}$, action $a_{1}^{\prime}$ dominates action $a_{2}^{\prime}$, but the strategy $\left(a_{1}, a_{2}^{\prime}\right)$ is I-admissible at $D_{1}$.

In this paper we adopt the consequentialist position that the best substrategy starting at a decision node can depend only on the subtree starting at that node, motivated by the fact that the sensible alternative, resolute behavior, is computationally unfeasible in general for bounded agents (in a sequential decision problem, the resolute behavior can be viewed as a "bruteforce" method, that demands the enumeration of all possible strategies).

\subsection{Selecting strategies}

Algorithm 5 presents a general framework for consequentialist sequential decision making in the presence of indeterminacy/imprecision in probabilities - that is, under partially ordered preferences. The algorithm can be specialized by replacing throughout CRITERION by the desired criterion of choice. The intuition behind the algorithm is that a strategy can

\footnotetext{
4 Nevertheless, both E-admissibility and Maximality still may lead to inconsistent choice, where the decision maker plans for an action but then executes a different action.
} 


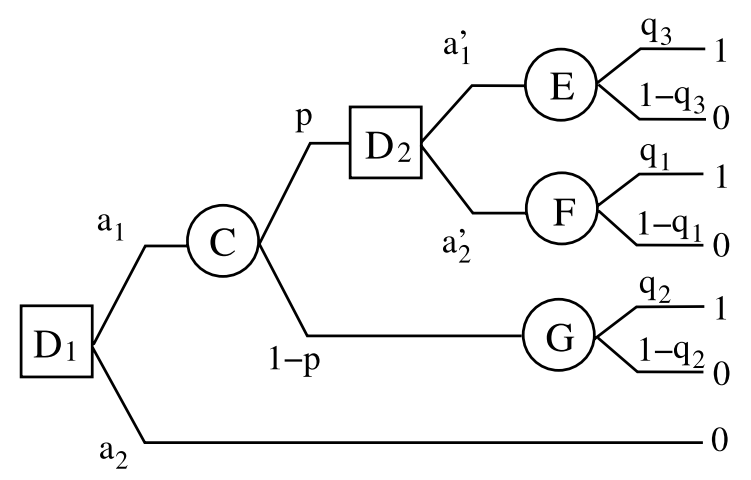

Fig. 5. Decision tree for Example 6.

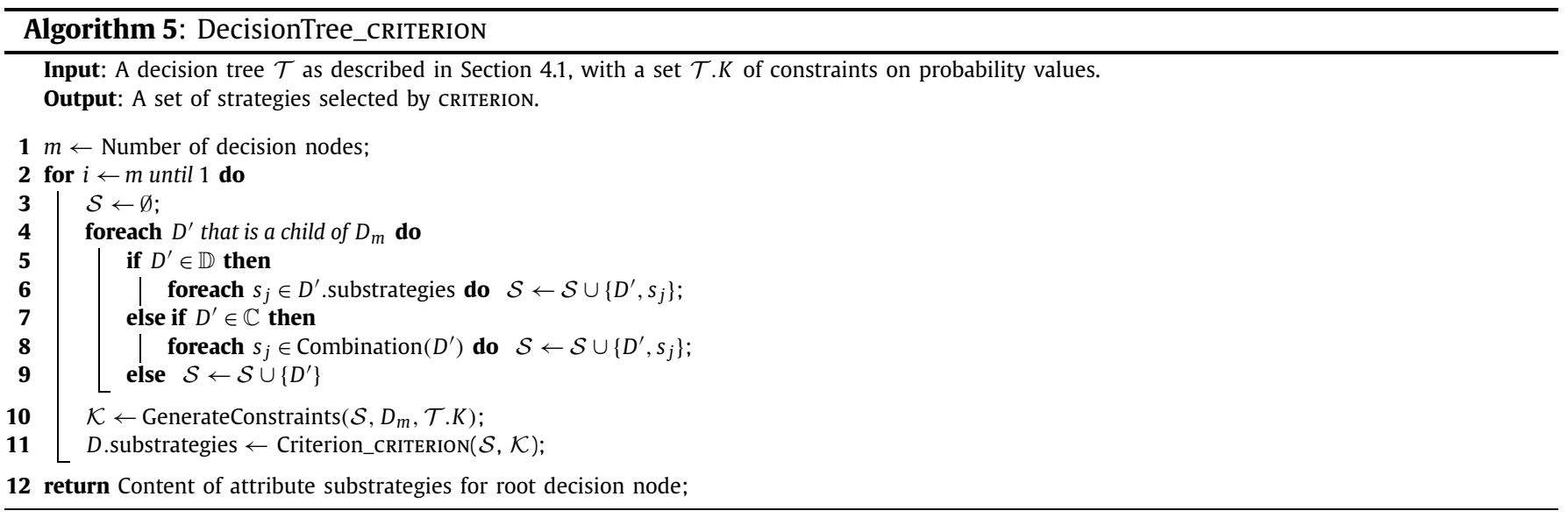

be constructed by visiting the nodes backwards, i.e. from last (leaf) to first (root), by selecting the optimal choice as decision nodes are encountered. The optimal choice at a decision node is then combined to the array of optimal choices selected at previously visited nodes, until the root node is resolved. In order to accomplish this, Algorithm 5 assumes that each decision node keeps a list of admissible substrategies (according to CRITERION) rooted at that node; this list is kept in the "substrategies" attribute. The remainder of this section is dedicated to a detailed discussion of technical aspects of this algorithm. In addition, we propose a simple transformation that allows the algorithm to be solved using linear programming instead of the more computationally demanding multilinear program. We also show that this linear program can benefit from the use of the column generation technique in order to obtain solutions more efficiently.

We assume that the decision nodes in $\mathbb{D}$ are topologically sorted, that is, they follow a linear (temporal) ordering of decisions such that if $\mathcal{T}$ contains a path from decision node $D_{x}$ to $D_{y}$, then $D_{x}$ appears before the decision node $D_{y}$ in the ordering. We assume that $D_{1}$ is the root of $\mathcal{T}$ and $D_{m}$ is the last decision node in such ordering.

As mentioned, the construction of strategies in our algorithm starts backwards at a leaf of the tree, that is, at a utility node (line 9 of Algorithm 5). We visit each decision node $D_{m}$ (from last to first) and examine its children. If a child $D^{\prime}$ of $D$ is a decision node, the substrategies rooted at $D^{\prime}$ are combined with the action that prescribes a move to $D^{\prime}$ (this is indicated by storing $D^{\prime}$ at the beginning of the substrategies). If $D^{\prime}$ is a chance node, there are two cases to handle. If a chance node has no decision nodes as successors, the only substrategy rooted at $D$ is a substrategy that simply moves to $D^{\prime}$. If a chance node has decision nodes as successors, it is then necessary to combine all substrategies that can branch out of $D^{\prime}$. For instance, if there are three decision nodes out of a chance node, and each one of these decision nodes leads to two substrategies, then eight substrategies must be produced. We assume that these substrategies are returned by the function Combination in line 8 of Algorithm 5.

The substrategies $\mathcal{S}$ generated in the loop from line 4 to line 9 are fed to the appropriate function Criterion_CRITERION in line 11. This function treats each substrategy as an action, and it must also receive the constraints and parameters of optimization programs that are run so as to select substrategies in Criterion_CRITERION. These constraints are basically contained in the input set $\mathcal{T} . K$, and given in terms of local assignments.

Once the constraints are generated, they are processed by Criterion_CRITERION in a sequence of optimization programs. Fix a decision node $D$ and strategy $s$; the expectation of $s$ is:

$$
\sum_{x_{1}, \ldots, x_{N} \in\{0,1\}} P\left(X_{1}=x_{1} \mid \operatorname{an}\left(X_{1}\right)\right) \ldots P\left(X_{N}=x_{N} \mid \operatorname{an}\left(X_{N}\right)\right) U\left(s, x_{1}, \ldots, x_{N}\right),
$$

where $X_{i}$ denotes indicator functions for each one of the events in the subtree rooted at $D$ only with branches selected by $s, \operatorname{an}\left(X_{i}\right)$ is the set of nodes in the path from $D$ to $X_{i}$, and $P\left(X_{i}=x_{i} \mid \operatorname{an}\left(X_{i}\right)\right)$ is a local assignment on the probability 
that the event $X_{i}$ obtains $x_{i}$. This expression is clearly in a multilinear form, and optimizing it subject to constraints on probability values (the terms in the product) takes us to nonlinear programming, as has been pointed out before [14,15,42]. Nonlinear programming is known to be a class of very difficult problems to solve, and specific optimization methods have been recently applied to the particular case of sequential decision making under the $\Gamma$-Maximax criteria by de Campos and Ji [19].

There are, however, interesting situations where all optimization programs in Criterion_CRITERION can be transformed into linear programs, as discussed in the next section.

\subsection{Selecting strategies with linear programming}

A linear formulation is obtained for strategy selection when assessments are linear constraints and are separately specified in the sense that probability constraints for the event at a particular chance node do not depend on probability values for any other event. For instance, assessments are separately specified if $\mathcal{T} . K$ contains only bounds on probabilities such as $P(A \mid B) \in[\alpha, \beta]$ where $A$ is an event at a chance node and $B$ is a conjunction of events in the decision tree from the root to that node (as illustrated by Example 6).

To obtain linear programs, we first note that Expression (2) can be written as

$$
\sum_{x_{1}, \ldots, x_{N} \in\{0,1\}} P\left(X_{1}=x_{1}, \ldots, X_{N}=x_{N}\right) U\left(s, x_{1}, \ldots, x_{N}\right),
$$

where $P\left(X_{1}=x_{1}, \ldots, X_{N}=x_{N}\right)$ are the probability values to optimize over. For instance, consider again constraints such as $P(A \mid B) \in[\alpha, \beta]$, where $A$ and $B$ are (conjunctions of) events in the decision tree. We can use Bayes' rule of conditioning to transform these constraints into the form $\alpha P(B) \leqslant P(A \cap B) \leqslant \beta P(B)$ under the assumption that probabilities are strictly positive. In Example 6, we have the constraints:

$$
\begin{aligned}
& 1 / 10 \leqslant P(C) \leqslant 3 / 10, \quad 3 / 5 P(C) \leqslant P(E \cap C) \leqslant 4 / 5 P(C), \\
& 1 / 5 P(C) \leqslant P(F \cap C) \leqslant 2 / 5 P(C), \quad 2 / 5 P\left(C^{C}\right) \leqslant P\left(G \cap C^{C}\right) \leqslant 3 / 5 P\left(C^{C}\right) .
\end{aligned}
$$

It is instructive to analyze the size of the linear programs that are generated by this method. One extreme (favorable) situation is represented by a symmetric decision tree where each decision node in the same slice branches into a constant number of chance nodes containing the same event. That is, the first decision node branches into several nodes containing event $A$; then the decision nodes out of these two chance nodes branch into several chance nodes all labeled with event $B$, and so on, as depicted in Fig. 6 (left) for the case of branching factor equal to two. For a fixed strategy, we have a symmetric binary tree, and if the problem deals with $N$ events, this binary tree contains $2^{N}-1$ nodes. Each complete path from the root to a utility node corresponds to a complete conjunction of events and complements of events, and the linear program to be built has as many optimization variables as there are paths in this symmetric binary tree. We reach the satisfying conclusion that, for symmetric decision trees, the linear programs that must be solved within Algorithm 5 are polynomial on the size of the decision tree (this is of course not entirely comforting as the size of these decision trees is exponential on the number of events). Fig. 6 (right) shows running times for the computation of lower expected value for a given strategy, as this is the basic operation for all criteria of choice. Points in that graph have been produced by generating symmetric decision trees with randomly generated utilities and lower/upper probabilities for events. The implementation is coded in AMPL and uses the CPLEX commercial package as linear programming solver; experiments were run in a microcomputer with two dual-core processors and 4 GBytes of memory. One sees that running times are quite small for $N \leqslant 10$; it is hard to imagine a symmetric decision tree with more than 10 chance nodes. Just to compare, we have also solved the multilinear formulation (2) using the programming package Multilin [17]. The multilinear programs took minutes even for $N=5$ and often failed to converge (Multilin produces successive approximations and typically reaches a vicinity of the solution quickly, but then converges very slowly).

Now consider the other extreme situation, where for every fixed strategy we have a symmetric binary tree such that each chance node contains a different event. Here the linear program that computes the lower/upper expectation of a strategy is exponentially larger than the decision tree: for a binary tree with height $H$, there are $N=2^{H}-1$ chance nodes and $2^{N}=2^{\left(2^{H}-1\right)}$ optimization variables. That is, we may have a relatively small decision tree that leads to very large linear programs: for example, in a binary tree of height 5 ( 5 levels), there are $N=2^{5}-1=31$ chance nodes and $2^{N}=2^{31}$ optimization variables. As indicated in Fig. 6 (right), running times grow substantially as $N$ grows beyond 10 . Here we face a situation that is similar to probabilistic logic; that is, we have a relatively small set of constraints on $N$ events and we must handle $2^{N}$ configurations of these events [24,27,31]. Such problems have been tackled with column generation [39] and redundancy detection $[32,48]$. We are interested in minimizing/maximizing an objective function given by Expression (3). We can write this expression as a product of vectors $u \cdot p$, where $u$ contains the values of the utility nodes and $p$ the probability values over the $2^{N}$ possible configuration of events. We also know from $\mathcal{T} . K$ the constraints $p$ is subject to, that can also be easily written in matrix form by $\mathbf{A} p \geqslant \mathbf{b}$.

It is clear that $\mathbf{A}$ has a very large number of columns (more precisely, $2^{N}$, one for each possible configuration of events). Storing and manipulating such an amount of columns is very inefficient and time consuming. However, by using column 

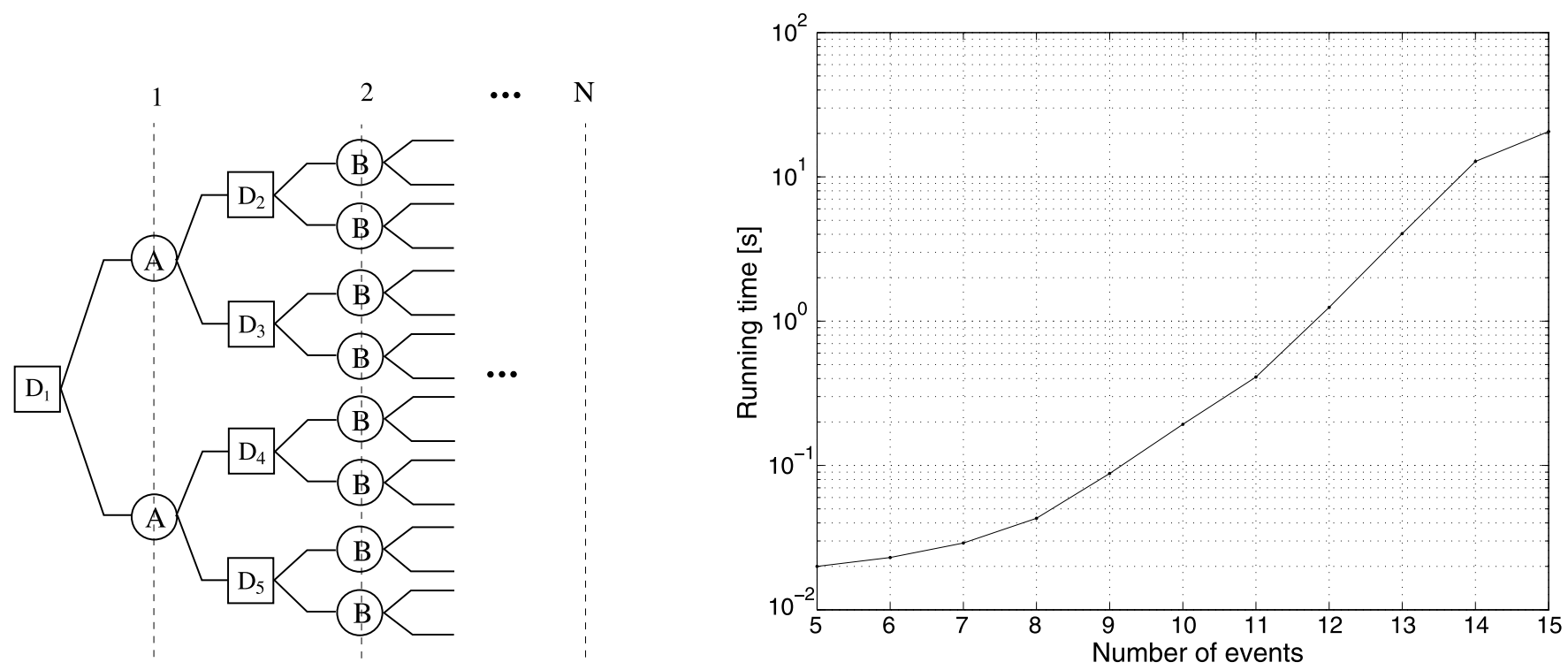

Fig. 6. Linear programming solution for exponentially large, separately specified decision trees. Left: Symmetric decision tree. Right: Running times, in logarithmic scale, for growing $N$ (number of events).

generation, it is possible to solve this linear program by manipulating only a smaller sub-matrix $\mathbf{A}^{\prime}$ of $\mathbf{A}$ with as many rows and columns as there are rows in $\mathbf{A}$. The challenge is that, to run the simplex method, at each iteration we must select one of the previously discarded columns of $\mathbf{A}$ to replace an existing column in $\mathbf{A}^{\prime}$. This is done by computing the reduced cost $c-y \mathbf{A}$, where $y$ is the dual cost of the current solution [4]. We can write every column of $\mathbf{A}$ as a vector of multilinear expressions $\left[A_{1} B_{1}-\alpha_{1} B_{1}, \ldots, A_{m} B_{m}-\alpha B_{m}\right]^{T}$, where $A_{i}$ is an event and $B_{i}$ is a conjunction of events, and $\alpha_{i}$ is an assessment. Thus $c-y \mathbf{A}$ is a multilinear expression on optimization variables that are either 0 or 1 ; there are standard techniques to reduce such an optimization problem to integer programming [18, Section 4.2]. To summarize: we run the simplex method with $\mathbf{A}^{\prime}$, and to decide which column of $\mathbf{A}$ to enter into $\mathbf{A}^{\prime}$, we run an auxiliary integer program. We note that problems with hundreds of variables in probabilistic logic have been solved using column generation with relative ease [32], so the exact solution of large decision trees can be obtained. Note also that these techniques can be extended to chance nodes that are associated with random variables with finitely many values. The added effort is to binarize the random variables into sets of binary variables, and to add Boolean constraints so that these binary variables only take on possible values. The result is again a probabilistic logic problem that can be solved using linear programming, possibly with column generation if $N$ is large.

\subsection{Consequentialist backward induction...?}

One might argue that constraints $\mathcal{K}$ should be generated only once before any other computation in Algorithm 5 , as assessments $\mathcal{T} . K$ are available as input. However such an approach may miss significant simplifications, because there may be chance nodes that are discarded during execution of the algorithm, given our consequentialist perspective. For instance, in Fig. 5 the event $F$ can be discarded when one is at $D_{1}$, because action $a_{2}^{\prime}$ and the subsequent nodes are not admissible for any criteria of choice previously discussed. Thus it makes sense to generate constraints "inside" the loop (line 10) in Algorithm 5. Nevertheless, in the worst case the function GenerateConstraints may build, if implemented as described in the previous section, an exponentially large optimization program at the root node. This is somewhat unsatisfying, particularly when compared to backward induction in standard decision trees.

In a standard decision tree, an already processed decision node is completely summarized by the unique expected value of the selected substrategy from that node on. Instead in the function GenerateConstraints described in the previous section, the programs built at decision nodes grow in size. The natural question is: Can we have a function GenerateConstraints that summarizes the already processed decision nodes through an interval of expected utility? For instance, in Example 6 we would like the choice between $a_{1}$ and $a_{2}$ to be resolved at $D_{1}$ only by processing an expectation interval from $D_{2}$. Alas, such an interval-based backward induction fails in general:

Example 7. Consider the decision tree in Fig. 7, adapted from Hammond [30]. Here $p \in[\epsilon, 1-\epsilon]$ and $q=\epsilon$, for some small $\epsilon>0$. Actions $a$ and $b$ are maximal and E-admissible at $D_{2}$, and $c$ and $d$ are maximal and E-admissible at $D_{3}$, but strategy $\left\{a^{\prime}, a\right\}$ dominates $\left\{a^{\prime \prime}, d\right\}$, and $\left\{a^{\prime \prime}, c\right\}$ dominates $\left\{a^{\prime}, b\right\}$. If $D_{2}$ and $D_{3}$ were to return the expectation intervals for their maximal/E-admissible actions, it would not be possible to detect that some strategies are dominated; in fact, all four strategies have overlapping expectation intervals. 


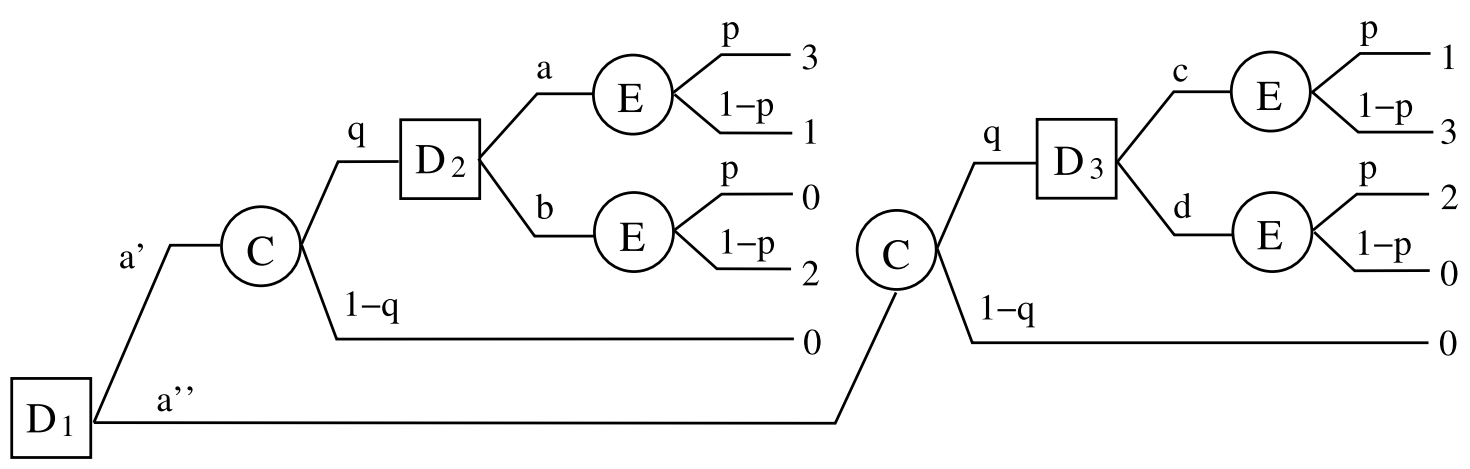

Fig. 7. Decision tree for Example 7.

Maximality and E-admissibility may fail in an interval-based backward induction because the necessary information about the constraints on probability values may be lost. We now wish to show that an interval-based backward induction can succeed for $\Gamma$-Maximin, $\Gamma$-Maximax, $\Gamma$-Maximix and Interval Dominance. That is, for these criteria and under the assumption that constraints are separately specified, we can evaluate actions at a decision node $D$ as a one-step decision problem where each decision node reached from $D$ is replaced by a single expectation interval. We start by rehearsing, in the next paragraph, an argument by Danielson and Ekenberg [14], who derived algorithms for computation of lower/upper expectations of a fixed strategy in the presence of local bounds on probabilities and expectations.

The central idea by [14] is as follows. Fix a strategy s; using Expression (2),

$$
E[s]=\min _{P_{1}, \ldots, P_{N}} \sum_{X_{1}} \cdots \sum_{X_{N}} P_{1}\left(X_{1} \mid \operatorname{an}\left(X_{1}\right)\right) \ldots P_{N}\left(X_{N} \mid \operatorname{an}\left(X_{N}\right)\right) U\left(s, X_{1}, \ldots, X_{N}\right),
$$

where we have subscripted the probability distributions so as to emphasize the scope of the minimization. We can move the summations to the right, eliminating variables one by one from $X_{N}$ to $X_{1}$, and we can place the minimization over a probability distribution right before the probability distribution of interest; this is only possible because all constraints are local and independently specified for each path from root to the node of interest. Hence to compute the lower expectation of $s$, we can run a backward induction scheme where a reduced optimization program is built at each decision node $D$ by encoding constraints in the subtree rooted at $D$, with action selected by $s$, and with branches cut at future decision nodes. These latter decision nodes are replaced by their lower and upper expected utilities; and as $D$ produces its own lower and upper expected utilities, it passes only these values back to its ancestors. Reduced programs are multilinear due to the presence of probability constraints and expectation intervals; that is, in Expression (2) we have both values of $P_{i}$ and of $U$ as free variables to optimize for. Danielson and Ekenberg [14] present techniques that simplify the solution of these local multilinear programs.

Returning to our problem, consider the criterion of Interval Dominance. At decision node $D$ we can build a complete optimization program with all chance nodes in the subtree rooted at $D$, except those nodes in subtrees eliminated in previous stages of the backward induction method. Alternatively, we can build a reduced optimization problem with the chance nodes between $D$ and the decision nodes that are direct descendants, with the proviso that these descendants summarize the content of their subtrees by intervals of expected utility. Recall that each decision node that is direct descendant of $D$ is attached to a fixed set of substrategies, because our decision maker is consequentialist. Thus the only question is whether the set of I-admissible actions at $D$ is the same regardless of whether we use the complete or the reduced program. Given that all that matters for Interval Dominance are lower/upper expectations, the argument in the previous paragraph leads to a positive answer: the two programs produce identical results, as the complete program can be divided into several smaller programs that are all encoded in the reduced program and its expectation intervals.

Similar arguments work for $\Gamma$-Maximin, $\Gamma$-Maximax and $\Gamma$-Maximix. Each descendant $D^{\prime}$ of $D$ is attached to a single selected substrategy $s^{\prime}$ rooted at $D^{\prime}$. Every expected utility in the expectation interval for $s^{\prime}$ can be attained by selecting probability distributions in the subtree rooted at $D^{\prime}$. Consequently, at $D$ we lose nothing by restricting attention to the expectation interval of $s^{\prime}$, and likewise for every descendant of $D$.

To finish this section, we compare several kinds of assessments and criteria of choice in the context of Example 3:

Example 8. Consider Example 3, and suppose $\epsilon$ is small. Algorithm 5 starts at $D_{2}$, where three substrategies, corresponding to actions $a_{1}^{\prime}, a_{2 a}^{\prime}$ and $a_{2 b}^{\prime}$, are evaluated by the appropriate function Criterion_CRITERION. The same happens at node $D_{3}$. At node $D_{1}$, the actions $a_{1}, a_{2 a}, a_{2 b}$ are evaluated, together with all combinations of selected strategies from $D_{2}$ and $D_{3}$. We have the implicit constraint that a single value $p$ refers to several probability values $\left(P\left(E \mid C, D_{1}\right), P\left(E \mid C^{C}, D_{2}\right)\right.$, etc.); that is, constraints are not separately specified. Running the complete backward induction algorithm, we obtain the following selected strategies. The $\Gamma$-Maximin actions are $a_{1}^{\prime}$ and $a_{1}^{\prime \prime}$ at $D_{2}$ and $D_{3}$, and the $\Gamma$-Maximin strategy is either $\left(a_{2 a}\right)$ or $\left(a_{2 b}\right)$ at $D_{1}$. (Note that action $a_{2 a}^{\prime}$ is inadmissible at $D_{2}$, action $a_{2 b}^{\prime \prime}$ is likewise inadmissible at $D_{3}$, but their combination is identical to $a_{2 a}$, a $\Gamma$-Maximin action at $D_{1}$ !) The $\Gamma$-Maximax criterion prescribes $a_{2 a}^{\prime}$ and $a_{2 b}^{\prime \prime}$ at $D_{2}$ and $D_{3}$, and then 
$\left(a_{3}, a_{2 a}^{\prime}, a_{2 b}^{\prime \prime}\right)$ at $D_{1}$. Interval Dominance selects all actions at nodes $D_{2}$ and $D_{3}$, so we have twelve strategies to evaluate at $D_{1}$, five of which are inadmissible $\left(\left(a_{1}\right),\left(a_{2 a}\right),\left(a_{2 b}\right),\left(a_{3}, a_{1}^{\prime}, a_{1}^{\prime \prime}\right)\right.$ and $\left.\left(a_{3}, a_{2 b}^{\prime}, a_{2 a}^{\prime \prime}\right)\right)$. E-admissibility discards only $a_{1}^{\prime}$ and $a_{1}^{\prime \prime}$ at $D_{2}$ and $D_{3}$, while Maximality does not discard any of them. At $D_{1}$ Maximality discards the same strategies as Interval Dominance plus the strategies $\left(a_{3}, a_{1}^{\prime}, a_{2 a}^{\prime \prime}\right)$ and $\left(a_{3}, a_{2 a}^{\prime}, a_{1}^{\prime \prime}\right)$. Finally, at $D_{1}$ E-admissibility discards the same strategies as Maximality plus the strategies $\left(a_{3}, a_{1}^{\prime}, a_{2 b}^{\prime \prime}\right)$ and $\left(a_{3}, a_{2 a}^{\prime}, a_{1}^{\prime \prime}\right)$. There are three E-admissible strategies: $\left(a_{3}, a_{2 a}^{\prime}, a_{2 a}^{\prime \prime}\right)$, $\left(a_{3}, a_{2 a}^{\prime}, a_{2 b}^{\prime \prime}\right)$, and $\left(a_{3}, a_{2 b}^{\prime}, a_{2 b}^{\prime \prime}\right)$.

Suppose we change Example 3 so that the probability of $E$ depends on the path from root to the chance node labeled with $E$; that is, we have eight probability values $p_{i} \in[0.25,0.75]$ (constraints are separately specified). A linear programming solution is now possible. However, if we wish we can deal with reduced programs through multilinear programming. Note that we face a decrease in selectivity by separating assessments: the only inadmissible strategy for Interval Dominance, Maximality and E-admissibility is $\left(a_{1}\right)$.

To summarize the discussion on decision trees: the same strategies are selected under resolute and consequentialist norms for Maximality and E-admissibility, and there, a backward induction procedure is fully justified; for the remaining criteria, a backward induction method is only justified by a consequentialist position (as adopted in this paper). Another point is that the size of optimization programs generated by Algorithm 5 may grow exponentially; however for $\Gamma$-Maximin, $\Gamma$-Maximax, $\Gamma$-Maximix and Interval Dominance it is possible to run reduced programs when constraints are separately specified. And finally, a linear programming formulation is possible in some cases but multilinear programming is required in general, and in particular when dealing with reduced programs.

\section{Influence diagrams with partially ordered preferences}

Decision trees can hardly represent large, or even medium size, decision problems, as the number of nodes in a decision tree increases exponentially with the number of chance and decision variables. A more compact way to represent sequential decision problems is through influence diagrams. A seminal work on influence diagrams with interval probabilities was presented by Breese and Fertig [8,22]; no substantial advance seems to have appeared in the literature after that work. We now expand that analysis by considering several criteria of choice.

The section is organized as follows. In Section 5.1 we introduce influence diagrams with partially ordered preferences, we define a class of problems that we are interested in, we present an algorithm to solve such class of problems and provide an analysis of complexity for the algorithm. In Section 5.2 we discuss several examples and experiments.

\subsection{Preliminaries, strategy selection and algorithm}

An influence diagram with imprecise probabilities is a directed acyclic graph over a set of decision nodes $\mathbb{D}$ (square shaped), chance nodes $\mathbb{C}$ (circle shaped) and utility nodes $\mathbb{U}$ (diamond shaped). Edges into a chance node indicate stochastic dependence; edges into a decision node indicate the available information at the time of the decision; edges into a utility node indicate functional dependence. Each decision node is associated with a finite set of actions conditional on its parents. Each chance node is associated with a random variable $C$ and with a set of credal sets: for each instantiation $\pi_{i}$ of the parents of $C(\mathrm{pa}(C))$, we have a credal set $K\left(C \mid \mathrm{pa}(C)=\pi_{k}\right)$ specified as in credal networks ${ }^{5}$; each utility node $U$ is associated with a function $u(\mathrm{pa}(U))$ that depends only on the parents of $U$. If more than one utility node is specified, then the total utility is the sum of all functions in utility nodes [68]. The standard definition of influence diagrams [34] requires a linear temporal order of all decisions (typically represented by a directed path comprising all decision nodes) and the no forgetting assumption, that is, at each decision node the decision maker knows all her previous decisions and past observations. However, some past information may be irrelevant and should not be considered for computational reasons [53,64]. In Limited Memory Influence Diagrams (LIMIDs) [44], the no forgetting assumption is relaxed, that is, the decision maker knows only the past decisions and observations that are explicitly linked to the decision nodes. This allows the representation of a broad class of decision problems, including situations with many decision makers.

A policy $\delta_{D}$ for decision node $D$ is a mapping from the parents of $D$ to the possible actions in $D$. A strategy $s$ is an ordered set of prescribed actions for all decision nodes, where each action depends on the parents of the decision node; that is, an ordered set of policies $s=\left\{\delta_{D_{1}}, \ldots, \delta_{D_{n}}\right\}$. The expression of expected utility for a strategy $s$, for a fixed probability distribution $P$, is

$$
\sum_{U \in \mathbb{U}, X \in\{\mathbb{C}, \mathbb{D}\}}\left(u(\operatorname{pa}(U)) \prod_{X} P(X \mid \operatorname{pa}(X))\right),
$$

where we note that if $X \in \mathbb{D}$, then its value is fixed by strategy $s$ (the variable is associated to zero/one probabilities given $s$ ). In standard influence diagrams and standard LIMIDs, an optimal strategy is a strategy with maximum expected utility (such strategies are called global maximum strategies in LIMIDs [44]).

It is important to pause for a moment and consider the properties of LIMIDs. First, any influence diagram is a LIMID where a decision node is informed about all previous decisions. However, if a LIMID contains a small number of arcs into

\footnotetext{
5 In this paper all variables have finitely many values.
} 


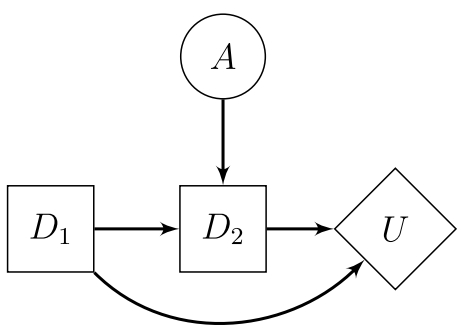

Fig. 8. A simple influence diagram with too many strategies.

decision nodes, the number of possible strategies is small when compared to the number of strategies in an influence diagram with identical graph. Hence the number of arcs into decision nodes is a critical parameter in a LIMID. Another important property of LIMIDs is that decision nodes are not necessarily ordered, so a decision maker contemplating a particular decision node may have no clue as to which decisions are implemented already and which decisions are still to be reached.

The lack of ordering amongst decisions in LIMIDs brings about a point that seems to have been missed in the literature. Namely, that LIMIDs are intrinsically inappropriate for consequentialist behavior. Clearly a decision maker can enumerate all strategies in a LIMID and then select a strategy with maximum expected utility, presumably to follow it all the way (in a resolute manner). A consequentialist behavior is harder to describe in the context of a LIMID. Suppose a decision maker seats at a decision node, considering only future moves in an attempt to evaluate its current decision; that is, in a consequentialist position. But how is this decision maker to know what are the "future" moves in a LIMID? There may be decisions that are not ordered with respect to the current decision, and the only way to examine the relative value of strategies is to consider all possible orderings. This may only be possible by considering the set of strategies from the outset, as a resolute decision maker would do. Indeed, the popular algorithm Single Policy Update (SPU) [44] finds nonoptimal strategies in LIMIDs by updating policies in some given order; the resulting strategies are not guaranteed to be optimal and, more importantly, the whole reasoning behind SPU cannot be given a consequentialist justification. For this reason, we do not attempt in this paper to adapt SPU to LIMIDs with imprecise probabilities; quite the contrary, we use a direct multilinear formulation of the strategy-selection problem. Note that a version of SPU for indecision-resistant criteria is not so difficult to conceive (as every decision node must yield a single policy) but a version of SPU for indecision-prone criteria seems not to be possible.

For these reasons, in this paper we are interested only in those LIMIDs that have a temporal order for decisions, so that we can always consider consequentialist behavior. Such an assumption about LIMIDs clearly limits the scope of models we can use, but it should be noted that the resulting class of LIMIDs is substantially larger than the class of influence diagrams; even though there is an ordering on decisions, the set of strategies that is allowed for a LIMID does not necessarily require each decision node to be aware of all previous decisions in the ordering. That is, the number of possible strategies in the LIMID may be substantially smaller than the number of strategies in an influence diagram of identical structure. In fact, the reason why we focus on "ordered" LIMIDs is exactly so that we can limit the number of possible strategies as compared to influence diagrams proper.

Example 9. Consider Fig. 8. Suppose that $A$ has four possible outcomes and $D_{1}$ has four possible actions. A policy for $D_{2}$ specifies one action for each configuration of the parents. If $D_{2}$ has two possible actions, then there are $2^{16}$ policies. In general, if we have $m$ configurations and $n$ actions, we can generate $n^{m}$ policies. This example shows that the number of policies grows quickly (exponentially) when decision nodes depend on several parents. In this case the search for optimal strategies may easily become intractable. For instance, suppose we intend to apply the Maximality criterion, then we have a total of $n^{m^{2}}$ optimization programs to evaluate.

As noted, our approach to strategy selection is to use a (consequentialist) backward induction process, rather than to resort to a variant of SPU or any other approximate scheme. The algorithm proceeds from the last decision node up to the first, building the strategies during execution by selecting admissible actions for each configuration of pa $(D)$, and then combining only the selected actions. The subtle point is that, for indecision-prone criteria, we may have to consider more than one admissible policy for a decision node $D$ as long as the algorithm iterates. This approach can save computations in two ways: (1) the computations are done locally (we do not need to consider all variables on the graph, thus, the optimization programs are smaller), and (2) if the number of selected actions is smaller than $n$, then we reduce the number of possible policies to be considered. Algorithm 6 summarizes this idea. Once the fact that we adopt a consequentialist position is understood, the algorithm can be viewed as a mix of the algorithm for policy selection in standard influence diagrams (where consequentialism is natural) and our previous algorithms for decision trees.

In Algorithm 6, we keep track of a list $\mathcal{S}_{i}$ associated to decision node $D_{i}$. The list $\mathcal{S}_{i}$ is used to hold the set of (sub)strategies suggested by indecision-prone criteria. It contains all admissible substrategies, evaluated by CRITERION, rooted at $D_{i}, \mathcal{S}_{i}=\left\{\delta_{D_{i}}, \ldots, \delta_{D_{m}}\right\}$. The initialization in line 2 indicates that previous to the first iteration there is no substrategy. As 


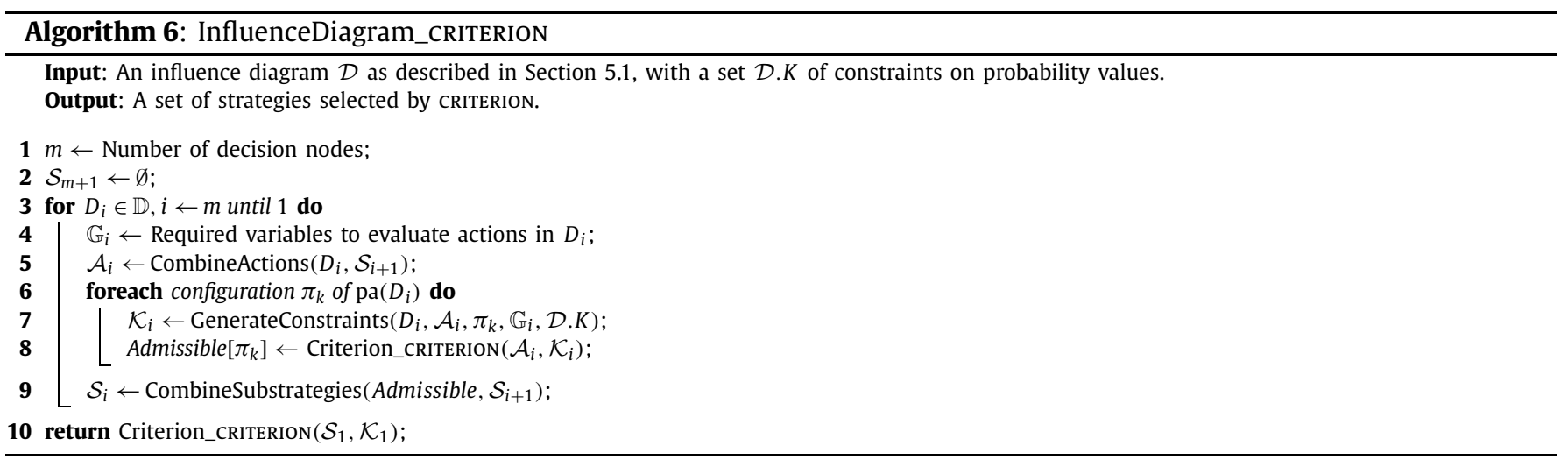

we have pointed out before, to evaluate an action in a given decision node, we do not need to consider all variables in the graph. This is exactly what we do in line 4 of Algorithm 6 . First we note that a utility node $U$ is relevant to a decision $D$ if there exists a directed path connecting $D$ and $U$. Then we use a standard d-separation algorithm [63] to obtain the needed variables, that is, the set of variables that are not d-separated from the set $\mathbb{U}_{i}$ of utility nodes relevant to $D_{i}$, given that the set $\left\{D_{i}, \mathrm{pa}\left(D_{i}\right)\right\}$ are "observed" (the decision node is clamped to the selected action). ${ }^{6}$ The function CombineActions takes the list of substrategies $\mathcal{S}_{i+1}$ and attaches an action of $D_{i}$ to each $s \in \mathcal{S}_{i+1}$. Suppose that in Example 9 there are two admissible policies in $D_{2}$, then at decision node $D_{1}$ the function CombineActions returns to $\mathcal{A}_{i}$ eight possible combinations. These combined actions will be evaluated by the criteria of choice before building the policies for decision node $D_{i}$. We rely on the criteria for reducing the number of admissible policies. This is done in the inner loop.

The function GenerateConstraints is responsible for creating the constraints on probabilities that are passed to the function Criterion_CRITERION. This function must encode the state space, as done for decision trees, and then encode constraints on probability values based on the input constraints $\mathcal{D}$.K. Differently from decision trees, the constraints in $\mathcal{K}_{i}$ must take into account the fact that lower/upper expectations are now: (1) restricted to variables in $\mathbb{G}_{i}$; and (2) conditional on a set of "observed" nodes $\left\{D_{i}, \operatorname{pa}\left(D_{i}\right)\right\}$. Thus the new element here is that we must minimize/maximize conditional expectations; this is done by introducing an auxiliary variable $z$ and a new constraint,

$$
\sum_{\mathbb{U}_{i}, \mathbb{G}_{i} \backslash \mathbb{E}_{i}} P\left(\mathbb{G}_{i}\right) u\left(\mathbb{U}_{i}\right)-z P\left(\mathbb{E}_{i}\right)=0,
$$

where $\mathbb{E}_{i}$ denotes the set of nodes "observed" at $D_{i}$. This constraint forces $z$ to be the desired conditional expectation. Hence the inner loop in Algorithm 6 builds the optimization programs as in decision trees, using Expression (5) in symbolic form whenever necessary.

The function CombineSubstrategies is responsible for building $\mathcal{S}_{i}$. It receives the set of admissible actions and the set of substrategies $S_{i+1}$, builds the possible policies for $D_{i}$, and appends them to the substrategies in $S_{i+1}$.

The complexity of the algorithm obviously depends on the criteria of choice. For indecision-resistant criteria, we always consider one optimal policy at each decision node and, consequently, we have only one strategy (similar to influence diagrams with precise probabilities). This is also the best case for indecision-prone criteria (when the criterion is very selective). The worst case happens when the criterion does not discard any action. This implies that we need to consider all possible combination of policies as long as we proceed in a backward fashion, and at the first decision node we have the same amount of strategies as a resolute decision maker.

\subsection{Examples and experiments}

The algorithm presented in the previous section has been implemented and run in several well-known examples. Most of the implementation was coded in the Java language, with calls to optimization packages (the multilinear programming package Multilin [17] and the CPLEX and Minos commercial packages respectively for linear and nonlinear optimization). Tests were run in a microcomputer with two dual-core processors and 4 GBytes of memory. We report the character of selected strategies and the computational effort spent to select these strategies.

We start with the classic oil wildcatter problem [56]; this is a small influence diagram, so we can indicate the steps of the algorithm in some detail.

Example 10. The oil wildcatter problem is depicted in Fig. 9. An oil wildcatter must decide whether to drill or not to drill (decision $D$ ). The cost of drilling is $\$ 70 \mathrm{~K}$. If the decision is to drill, the soil may be soaking, wet or dry (with a return

\footnotetext{
6 Due to their independence relations, influence diagrams and LIMIDs can be viewed as extended Bayesian networks [10,63,64]. Our framework can be viewed as an extended credal network, so d-separation applies.
} 


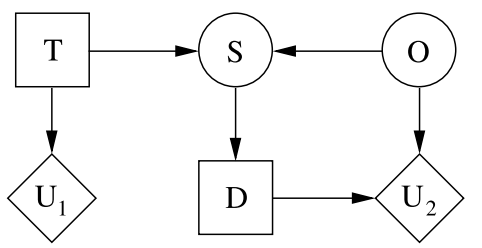

\begin{tabular}{c|ccc}
\multirow{2}{*}{ Test $(S)$} & Ary & Amount of oil $(0)$ \\
\hline ns & {$[0.60,0.65]$} & {$[0.30,0.35]$} & {$[0.10,0.10]$} \\
os & {$[0.25,0.30]$} & {$[0.40,0.40]$} & {$[0.40,0.45]$} \\
cs & {$[0.10,0.10]$} & {$[0.25,0.30]$} & {$[0.45,0.50]$}
\end{tabular}

Fig. 9. Influence diagram for the oil wildcatter problem and probability intervals for seismic test given amount of oil.

of $\$ 270 K, \$ 120 K$ or $\$ 0$ respectively). Suppose probabilities for the amount of oil $(0)$ are: $P(0=$ soaking $) \in[0.2,0.2]$, $P(O=$ wet $) \in[0.3,0.35], P(O=$ dry $) \in[0.45,0.5]$. At the cost of $\$ 10 K$, the oil wildcatter can take seismic soundings of the site. The result of this test $(S)$ may be ns (no oil), os (some oil), cs (abundance of oil), with interval probabilities in Fig. 9. If the test is not conducted, then $P(S=S \mid 0)=1$ if $\{S=\mathrm{nt}\}$ and $P(S \mid O)=0$ otherwise, where nt is a special value of $S$ that indicates absence of test.

To select strategies, we start with the decision $D$; node $U_{2}$ is required and all nodes but $U_{1}$ are returned by GetdConnected. The expected utility of not drilling is 0.00 regardless of $S$. The expected utility of drilling depends on $S$. To compute the lower expected utility of drilling given that $\{S=n s\}$, we must minimize auxiliary variable $z$ subject to constraints on probabilities and to

$$
\sum_{O} P(O) P(S=\mathrm{ns} \mid O, T) u(D=\text { yes, } O)-z P(S=\mathrm{ns})=0 .
$$

Running this and similar multilinear programs, we obtain:

\begin{tabular}{lllll}
\hline$S$ & nt & ns & os & cs \\
\hline$E[D=$ yes $\mid S]$ & 20.00 & -32.76 & 32.86 & 82.61 \\
$\bar{E}[D=$ yes $\mid S]$ & 26.00 & -21.27 & 50.00 & 91.29 \\
\hline
\end{tabular}

Using the $\Gamma$-Maximix criterion with $\eta=0.5$ we find that the action not to drill is not admissible, except when the seismic test indicates ns. Thus we have the policy $\delta_{D}^{*}(S)=$ ns if $\{S=$ no $\}$ and $\delta_{D}^{*}(S)=$ yes otherwise. As the $\Gamma$-Maximix criterion specifies only one policy in $D$, we have only two policies to analyze at $T\left(\delta_{T}=\right.$ yes and $\delta_{T}=$ no). The overall utility is given by the sum of $U_{1}$ and $U_{2}$; we then obtain $E[T=$ yes $] \in[-10 K,-10 K]+[31.75 K, 37.23 K]=[21.75 K, 27.23 K]$ and $E[T=$ no $] \in[0,0]+[20 K, 26 K]=[20 K, 26 K]$. The selected action at $T$ is to take the seismic test $\left(\delta_{T}^{*}=\right.$ yes $)$. The selected strategy is $s^{*}=\left\{\delta_{T}^{*}, \delta_{D}^{*}\right\}$. This strategy is also selected by the $\Gamma$-Maximin and $\Gamma$-Maximax criteria. With E-admissibility, we obtain at $D$ the same policy suggested by the $\Gamma$-Maximix criterion. At node $T$, we have two E-admissible policies, $\delta_{T}=$ yes and $\delta_{T}=$ no. The combination of optimal policies leads to two strategies with expected utilities respectively in $[21.75 \mathrm{~K}, 27.23 \mathrm{~K}]$ and $[20.0 \mathrm{~K}, 26.0 \mathrm{~K}]$. Interval Dominance and Maximality select the same strategies as E-admissibility.

The next influence diagram we examine is the "Breeding Pigs" problem described by Lauritzen and Nilsson [44], and represented by the influence diagram in Fig. 10. A pig breeder is growing pigs for a period of four months and subsequently selling them. During this period the pig may or may not develop a certain disease $\left(h_{i}\right.$ represents the pig's health, healthy or ill, in the $i$ th month). Once a month, a doctor makes a test for the presence of the disease $\left(t_{i}\right.$ represents the test's results, disease free or otherwise), and the doctor may or may not treat the pig for the disease by injecting a certain drug (decision node $d_{i}$ ). The utility nodes $u_{1}, u_{2}, u_{3}$ represent the cost of treating the pig, and $u_{4}$ represents the payoff for selling the pig. Additionally, we have: the price of a pig with disease is 300DKK (Danish kroner) and of a disease-free pig is $1000 D K K$ (utility node $u_{4}$ ); the cost of an injection is $100 D K K$ (utility nodes $u_{1}, u_{2}, u_{3}$ ); the test is correct when the pig is ill with probability 0.80 , and correct when the pig is healthy with probability 0.90 (chance nodes $t_{i}$ ); a healthy pig develops the disease in the subsequent month with probability 0.20 without injection, whereas a healthy and treated pig develops the disease with probability 0.10 ; an untreated pig that is unhealthy will remain so in the subsequent month with probability 0.90 , whereas the similar probability is 0.5 for an unhealthy pig that is treated (chance nodes $h_{2}, h_{3}, h_{4}$ ). The decision maker is uncertain about the pig's health in the first month $\left(h_{1}\right)$. In our experiments we assume two intervals for $h_{1}$ : the first one is a small interval defined near the probability of the original problem $(P(\mathrm{ill})=[0.1,0.2])$; the second one is a large interval $(P($ ill $)=[0.0,0.5])$.

In this example the full previous treatment and test history are available when decisions are made. At decision node $d_{3}$, there are 5 conditioning nodes. A policy at $d_{3}$ specifies an action for 32 configurations (all five conditioning nodes have just two possible values). An indecision-resistant criterion must run 64 inferences to find out the best policy, while an indecision-prone criterion may have to keep track of many incomparable substrategies. It is indeed possible that the large number of incomparable substrategies makes it impossible to finish the algorithm, as we will see in a moment.

Now, consider a LIMID for the Breeding Pigs problem, where the decision maker does not remember past decisions and results of tests from previous months (the decision maker remembers only the result of the test taken in the current month). The resulting LIMID is also depicted in Fig. 10. 

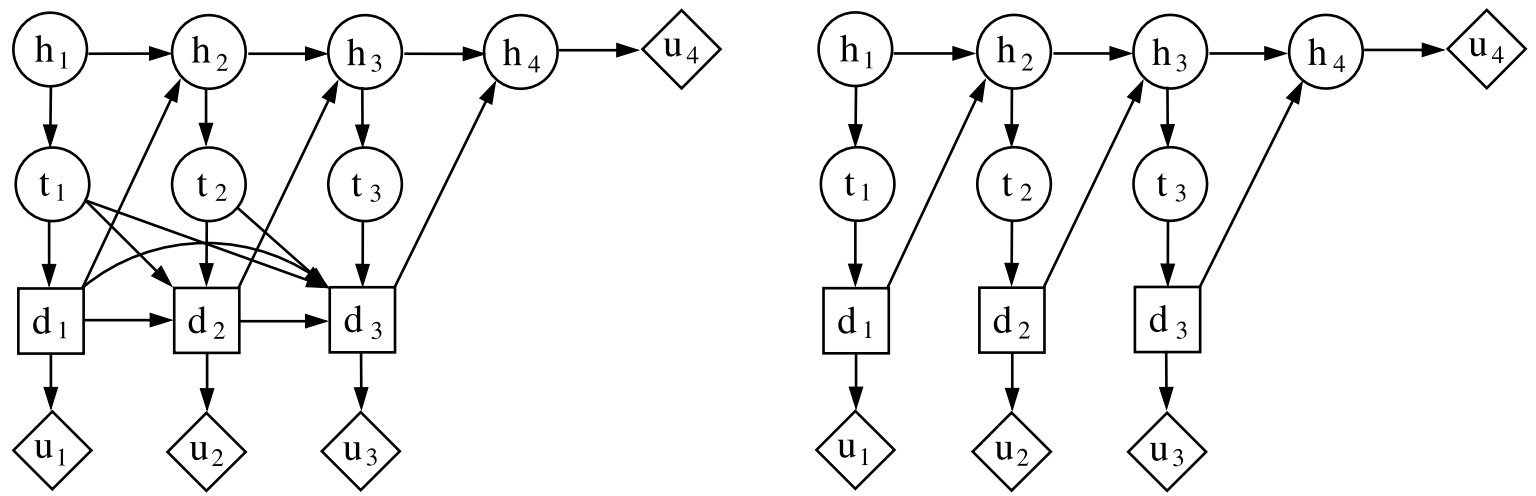

Fig. 10. Left: Influence diagram (with complete history) for the Breeding Pigs problem. Right: LIMID version for the Breeding Pigs problem.

Table 1

Experiments with the Breeding Pigs problem.

\begin{tabular}{|c|c|c|c|c|c|}
\hline$P\left(h_{1}=\right.$ ill $)$ & Criteria & $\begin{array}{l}\text { \# of admissible } \\
\text { strategies LIMIDs }\end{array}$ & $\begin{array}{l}\text { Elapsed time (s) } \\
\text { LIMIDs }\end{array}$ & $\begin{array}{l}\text { \# of admissible } \\
\text { strategies IDs }\end{array}$ & $\begin{array}{l}\text { Elapsed time (s) } \\
\text { IDs }\end{array}$ \\
\hline \multirow[t]{6}{*}[0.1,0.2]{} & $\Gamma$-Maximin & 1 & 2.41 & 1 & 10.20 \\
\hline & $\Gamma$-Maximax & 1 & 2.63 & 1 & 11.45 \\
\hline & $\Gamma$-Maximix & 1 & 3.59 & 1 & 16.03 \\
\hline & I. Dominance & 2 & 5.35 & 16 & 89.95 \\
\hline & Maximality & 1 & 1.84 & 2 & 17.59 \\
\hline & E-admissibility & 1 & 2.39 & 2 & 23.58 \\
\hline \multirow[t]{6}{*}[0.0,0.5]{} & $\Gamma$-Maximin & 1 & 3.74 & 1 & 16.48 \\
\hline & $\Gamma$-Maximax & 1 & 5.27 & 1 & 32.23 \\
\hline & $\Gamma$-Maximix & 1 & 7.57 & 1 & 43.39 \\
\hline & I. Dominance & 8 & 23.42 & - & - \\
\hline & Maximality & 2 & 3.76 & - & - \\
\hline & E-admissibility & 1 & 4.14 & 7 & 584.64 \\
\hline
\end{tabular}

Table 1 presents results for all criteria we have discussed. The column labeled \# of admissible strategies reports the number of strategies selected by our implementation, and the column labeled Elapsed time shows the average time of execution over thirty runs for each criterion. An interesting fact is that the smaller probability interval leads to smaller execution times: the sharper the probabilities, the smaller the number of incomparable substrategies to process. Another point to note is that Interval Dominance and Maximality could not be run to termination for the larger probability interval, due to the huge number of strategies that these criteria fail to discard during execution. A curious fact is that E-admissibility does not crash the system, and indeed leads to a relatively small number of selected strategies: even though E-admissibility seems more complex computationally, the fact that it is more selective than Interval Dominance and Maximality is quite valuable in practice.

Another curious fact in Table 1 is that, with the LIMID, Maximality and E-admissibility seem to be faster than the indecision-resistant criteria. This result can be traced to a few computational aspects that are not apparent from a superficial analysis. As we can define the set of maximal/E-admissible actions without computing exact probability values (just find a distribution satisfying the constraints), we use a fast approximated solver in Minos to produce a preliminary selection of actions. After this, we use the exact solver Multilin to compute lower/upper expectations. The approximated solver quickly gets close to the exact solution, so the overall computing time is greatly benefited. It is also possible to explain the weak showing of Interval Dominance, as it requires the use of the exact solver to compute lower and upper expectations so as to compare actions. ${ }^{7}$ It is noteworthy that Interval Dominance is a simple criterion to apply in single-stage decision problems while in sequential decision problems it faces difficulties due to its low selectivity.

Our final example deals with a relatively large LIMID that has been proposed by de Campos and Ji [19] as a model for Effects-Based Operation planning (EBO). The LIMID is shown in Fig. 11; all variables are binary, and the decision nodes have two possible actions (yes/no). The cost of actions, given by $\left\{U_{i}\right\}_{i=1}^{11}$, is: if $D_{i}$ is yes, then cost is 50 for $U_{3}, 150$ for $U_{8}, 80$ for $U_{10}, 100$ for $U_{11}$ and 20 for the all others. The reward for achieving the main goal is 1000 , while not achieving it costs 500 (utility node $U_{H}$ ). The chance nodes $C_{i}$ represent the rate of success with an interval probability $P\left(C_{i}=1 \mid D_{i}=\right.$ yes $) \in[0.9,1.0]$. The chance nodes $B_{j}$ and $A_{k}$ have probability 1 if all parents are positive, probability 0.5 if only one parent is positive, and probability 0 otherwise. The probability of chance node $G$ is 1 given that its parents

\footnotetext{
7 If we use the solver Minos to find I-admissible actions, Interval Dominance takes 2.68 and 56.70 seconds in the LIMID and influence diagram respectively, when $P\left(h_{1}=\right.$ ill $) \in[0.1,0.2]$; and it takes 9.45 seconds in the LIMID, and crashes with the influence diagram, when $P\left(h_{1}=\right.$ ill $) \in[0.0,0.5]$.
} 


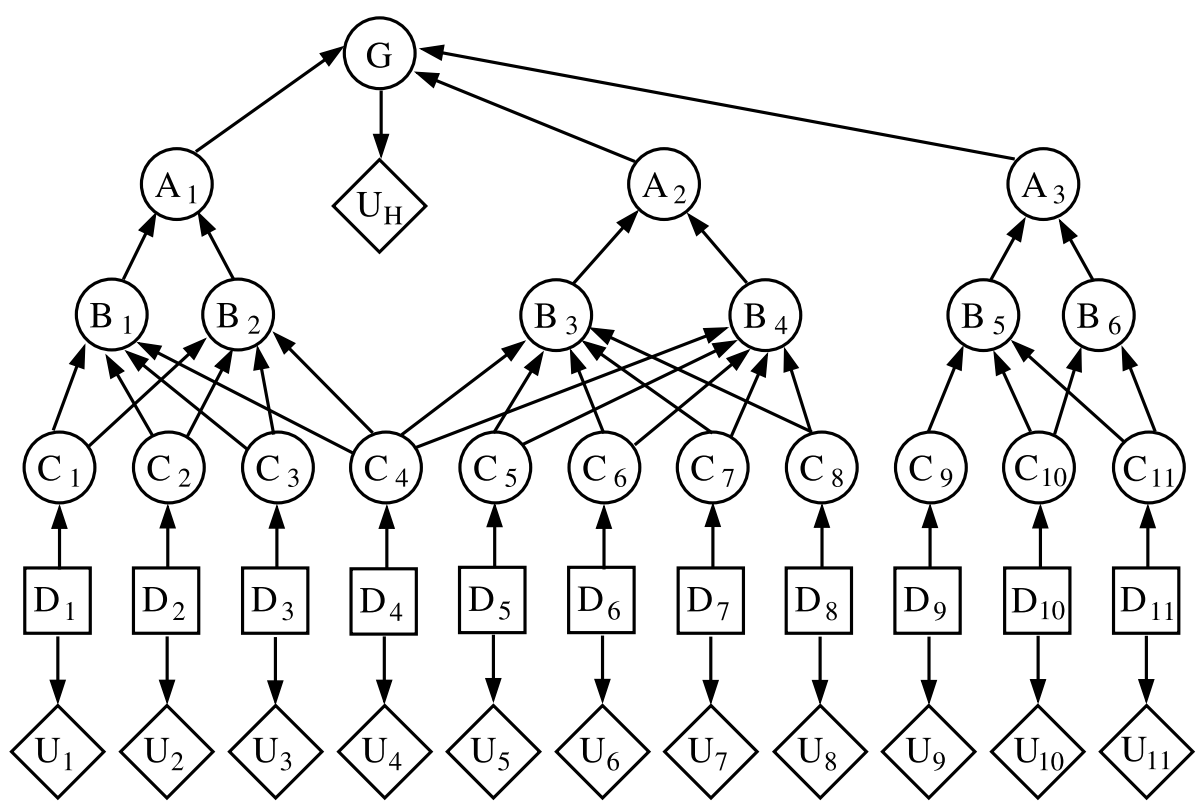

Fig. 11. Influence diagram for the EBO problem.

are positive, 0.6 if only one parent is negative, 0.3 if two parents are negative, and 0 otherwise. Decision nodes have no parents (an extreme LIMID), hence an arbitrary ordering of decision nodes is adopted when selecting strategies. Using the $\Gamma$-Maximin criterion, the selected strategy is to take action yes in all decision nodes except $D_{5}, D_{6}, D_{7}$ and $D_{8}$ (approximately 40 seconds were taken to select it). The lower expected utility of this strategy is -55.28 ; the elapsed time to select it was approximately 40 seconds. The only E-admissible strategy is to take action yes in all decision nodes except $D_{8}$ (approximately 120 seconds were taken to select it). The expected utility of this strategy belongs to [68.97, 330]; note that the consequentialist E-admissible strategy is always better than the consequentialist $\Gamma$-Maximin strategy. ${ }^{8}$

\section{Conclusion}

This paper has examined the selection of strategies in sequential decision making when preferences are partially ordered. In particular, we have focused on preference patterns that are encoded through a single utility and a set of probability measures. A partial order over strategies introduces subtle ingredients into the decision problem, as we have several criteria of choice and behavioral norms, episodes of incoherent/inconsistent choice, and varying degrees of computational gain. We have tried to shed some light into these matters from a consequentialist perspective, and to present algorithms that select strategies by solving sequences of optimization programs. Most algorithms employ multilinear programming, and some particular cases can be tackled by linear programming. Clearly, algorithms based on multilinear programming can be adapted to handle interval-valued utility, as we then have products between probabilities and utilities that must be optimized over; we have refrained from discussing interval-valued utility so as to limit the length of the paper.

The current literature on sequential decision making with partially ordered preferences can be roughly divided in two streams. The philosophical debate tends to favor abstract comparisons amongst criteria and norms, with little consideration of computational costs. On the other hand, if one looks at techniques that do involve decision making with partially ordered preferences, such as nondeterministic planning and CP nets, and even the theory of LIMIDs, one finds detailed study of computational costs but little attention to criteria, norms, and consistency. We hope that this paper strikes some needed balance between conceptual discussion and computational development, and helps shorten some of the gaps between these viewpoints. In particular we believe that the effect of the consequentialist perspective is currently not appreciated in the artificial intelligence literature, exactly where this norm is most adequate as one must deal with bounded agents.

We can summarize our contributions as follows. We have first derived new algorithmic techniques for Interval Dominance and E-admissibility (using insights by Kyburg and Pittarelli [43], as done independently by Utkin and Augustin [72]). We also presented a brief analysis of incoherent choice with Interval Dominance. More importantly, we have studied decision trees with partially ordered preferences, by presenting a consequentialist backward induction framework with multilinear and linear programming instantiations, and by noting that different criteria do affect the computational properties of backward induction. We have then applied these insights to influence diagrams, and actually to ordered LIMIDs, where the technology of credal networks (d-separation and multilinear programming) is used as much as possible. We have

\footnotetext{
8 By brute-force enumeration of strategies, we find that there is an E-admissible strategy with lower expected utility 156.41 (and upper expected utility 480.00); the consequentialist approach misses this possibility.
} 
also presented experiments with a complete implementation of the algorithms for influence diagrams. Given the lack of literature on influence diagrams with indeterminacy and imprecision in probability values, our results are a first step in understanding this powerful but intricate model. We have noted already that recently Huntley and Troffaes [35] and de Campos and Ji [19] have presented specialized algorithms respectively for Maximality in decision trees and for $\Gamma$-Maximax in influence diagrams, that can be more efficient than the algorithms discussed in this paper in particular problems.

An important subject this paper has not tackled is Markov Decision Processes (MDPs). MDPs are probably the most prominent model for sequential decision making under uncertainty in use in artificial intelligence today, and could likewise benefit from partial preference orderings. It should be noted that the initial translation into the framework of MDPs is not difficult, in fact there is a considerable amount of publications that deal with MDPs with sets of probability (usually referred to as Markov Decision Processes with Imprecise Probabilities) [33,58,74]. The topic deserves an investigation of its own; additionally, there are many questions that must be answered before partial preferences can be extensively used in MDPs, such as how to deal with act-state dependence and how to choose between the set of incomparable choices suggested by criteria such as Maximality and E-admissibility.

We certainly leave many avenues for future exploration. A necessary next step is a detailed empirical characterization of computational effort in solving decision trees and influence diagrams, including a comparison between multilinear and linear programming schemes whenever the latter are possible. It would also be important to characterize the class of influence diagrams that can be solved through reduced programs (that is, programs that deal with small subsets of chance and decision nodes, passing back interval-valued expected utility). There are also conceptual questions that deserve further scrutiny, as there are other criteria of choice and behavioral norms besides the ones investigated in this paper. Even within the scope of criteria discussed in this paper, there are questions to answer. For instance: we have produced algorithms that compute all E-admissible strategies; perhaps in practice one should be content with just one E-admissible strategy?

\section{References}

[1] M. Allais, O. Hagen, Expected Utility Hypotheses and the Allais Paradox, D. Reidel Publishing Company, Dordrecht, Holland, 1979.

[2] R.J. Aumann, Utility theory without the completeness axiom, Econometrica 30 (3) (July 1962) 445-461.

[3] J.O. Berger, Statistical Decision Theory and Bayesian Analysis, Springer, New York, 1985.

[4] D. Bertsimas, J.N. Tsitsiklis, Introduction to Linear Optimization, Athena Scientific, Belmont, Massachusetts, 1997.

[5] L. Blume, A. Brandeburger, E. Dekel, Lexicographic probabilities and choice under uncertainty, Econometrica 59 (1) (January 1991) 61-79.

[6] B. Bonet, R. Givan, in: 5th International Planning Competition: Non-deterministic Track, call for participation, 2005.

[7] C. Boutilier, R.I. Brafman, H.H. Hoos, D. Poole, CP-nets: A tool for representing and reasoning with conditional ceteris paribus preference statements, Journal of Artificial Intelligence Research 21 (2004) 135-191.

[8] J. Breese, K. Fertig, Decision making with interval influence diagrams, in: Sixth Conference on Uncertainty in Artificial Intelligence, Elsevier Science, New York, 1990, pp. 122-129.

[9] K. Bykvist, Time-partial morality and dynamic choice, in: W. Rabinowicz (Ed.), Value and Choice - Some Common Themes in Decision Theory and Moral Philosophy, Lund Philosophy Reports, 2000, pp. 53-64.

[10] G.F. Cooper, A method for using belief networks as influence diagrams, in: Proceedings of the 4th Conference on Uncertainty in Artificial Intelligence, Minneapolis, 1988, pp. 55-63.

[11] I. Couso, S. Moral, P. Walley, A survey of concepts of independence for imprecise probabilities, Risk, Decision and Policy 5 (2) (2000) $165-181$.

[12] F.G. Cozman, Separation properties of sets of probabilities, in: C. Boutilier, M. Goldszmidt (Eds.), Proceedings of the 16th Conference on Uncertainty in Artificial Intelligence, Morgan Kaufmann, San Francisco, July 2000, pp. 107-115.

[13] F.G. Cozman, Graphical models for imprecise probabilities, International Journal of Approximate Reasoning 39 (2-3) (June 2005) 167-184.

[14] M. Danielson, L. Ekenberg, Computing upper and lower bounds in interval decision trees, European Journal of Operational Research 181 (2) (September 2007) 808-816.

[15] M. Danielson, L. Ekenberg, J. Johansson, A. Larsson, The DecidelT decision tool, in: J.-M. Bernard, T. Seidenfeld, M. Zaffalon (Eds.), Proceedings of the 3rd International Symposium on Imprecise Probabilities and Their Applications, Carleton Scientific, Lugano, Switzerland, July 2003 , pp. $204-217$.

[16] C.P. de Campos, F.G. Cozman, Inference in credal networks using multilinear programming, in: Proceedings of the 2nd European Starting AI Researcher Symposium, IOS Press, Valencia, Spain, August 2004, pp. 50-61.

[17] C.P. de Campos, F.G. Cozman, The inferential complexity of Bayesian and credal networks, in: Proceedings of the 9th International Joint Conference on Artificial Intelligence, Edinburgh, Scotland, UK, July-August 2005, pp. 1313-1318.

[18] C.P. de Campos, F.G. Cozman, Inference in credal networks through integer programming, in: International Symposium on Imprecise Probability: Theories and Applications, Prague, 2007, pp. 145-154.

[19] C.P. de Campos, Q. Ji, Strategy selection in influence diagrams using imprecise probabilities, in: Proceedings of the 24th Conference on Uncertainty in Artificial Intelligence, Helsinki, Finland, July 2008, pp. 121-128.

[20] D. Ellsberg, Risk, ambiguity, and the Savage axioms, The Quarterly Journal of Economics 75 (4) (1961) 643-669.

[21] N. Etchart, Adequate moods for Non-EU decision making in a sequential framework, Theory and Decision 52 (February 2002 ) 1-28.

[22] K. Fertig, J. Breese, Probability intervals over influence diagrams, IEEE Transactions on Pattern Analysis and Machine Intelligence 15 (3) (1993) 280-286.

[23] P.C. Fishburn, Utility Theory for Decision Making, Kriefer Publishing Company, New York, 1970.

[24] G. Georgakopoulos, D. Kavvadias, C.H. Papadimitriou, Probabilistic satisfiability, Journal of Complexity 4 (1) (March 1988) 1-11.

[25] I. Gilboa, D. Schmeidler, Maxmin expected utility with non-unique prior, Journal of Mathematical Economics 18 (2) (1989) $141-153$.

[26] F.J. Giron, S. Rios, Quasi-Bayesian Behaviour: A More Realistic Approach to Decision Making? University Press, Valencia, 1980.

[27] T. Hailperin, Best possible inequalities for the probability of a logical function of events, American Mathematical Monthly 72 (1965) $343-359$.

[28] P.J. Hammond, Changing tastes and coherent dynamic choice, The Review of Economic Studies 43 (1) (1976) $159-173$.

[29] P.J. Hammond, Consequentialism and the independence axiom, in: B.R. Munier (Ed.), Risk, Decision and Rationality (Proceedings of the 3rd International Conference on the Foundations and Applications of Utility, Risk and Decision Theories), Dordrecht, Holland, 1988, pp. 503-516.

[30] P.J. Hammond, Orderly decision theory: a comment on Professor Seidenfeld, Economics and Philosophy 4 (1988) $272-297$.

[31] P. Hansen, B. Jaumard, Probabilistic satisfiability, Tech. Rep. G-96-31, Les Cahiers du GERAD, École Polytechique de Montréal, 1996.

[32] P. Hansen, S. Perron, Merging the local and global approaches to probabilistic satisfiability, International Journal of Approximate Reasoning 47 (2) (2008) 125-140. 
[33] D. Harmanec, Generalizing Markov decision processes to imprecise probabilities, Journal of Statistical Planning and Inference 105 (1) (June 2002) 199-213.

[34] R.A. Howard, J.E. Matheson, Influence diagrams, Decision Analysis 2 (3) (2005) 127-143.

[35] N. Huntley, M. Troffaes, An efficient normal form solution to decision trees with lower previsions, in: International Workshop on Soft Methods in Probability and Statistics, 2008, pp. 419-426.

[36] L. Hurwicz, A class of criteria for decision-making under ignorance, Cowles Comission Paper 356, 1951.

[37] H. Itoh, K. Nakamura, Partially observable Markov decision processes with imprecise parameters, Artificial Intelligence 171 (8-9) (2007) 453-490.

[38] J.-Y. Jaffray, Rational decision making with imprecise probabilities, in: G.D. Cooman, F.G. Cozman, S. Moral, P. Walley (Eds.), Proceedings of the 1st International Symposium on Imprecise Probabilities and Their Applications, Ghent, Belgium, June 1999, pp. 183-188.

[39] B. Jaumard, P. Hansen, M.P. de Aragão, Column generation methods for probabilistic logic, ORSA Journal on Computing 3 (2) (1991) 135-148.

[40] D. Kahneman, A. Tversky, Prospect theory: An analysis of decisions under risk, Econometrica 47 (1979) $262-291$.

[41] D. Kikuti, F.G. Cozman, Influence diagrams with partially ordered preferences, in: 3rd Multidisciplinary Workshop on Advances in Preference Handling, 2007.

[42] D. Kikuti, F.G. Cozman, C.P. de Campos, Partially ordered preferences in decision trees: Computing strategies with imprecision in probabilities, in: Workshop on Advances in Preference Handling, Edinburgh, United Kingdom, July 2005, pp. 118-123.

[43] H.E. Kyburg Jr., M. Pittarelli, Set-based Bayesianism, IEEE Transactions on Systems, Man and Cybernetics, Part A 26 (3) (1996) $324-339$.

[44] S.L. Lauritzen, D. Nilsson, Representing and solving decision problems with limited information, Management Science 47 (9) (2001) $1235-1251$.

[45] I. Levi, On indeterminate probabilities, The Journal of Philosophy 71 (1974) 391-418.

[46] I. Levi, The Enterprise of Knowledge, The MIT Press, Massachusetts, 1980.

[47] R.D. Luce, H. Raiffa, Games and Decisions, Wiley, New York, 1957.

[48] C. Luo, C. Yu, J. Lobo, G. Wang, T. Pham, Computation of best bounds of probabilities from uncertain data, Computational Intelligence 12 (4) (1996) 541-566.

[49] M.J. Machina, Dynamic consistency and non-expected utility models of choice under uncertainty, Journal of Economic Literature 27 (4) (December 1989) 1622-1688.

[50] E.F. McClennen, Rationality and Dynamic Choice: Foundational Explorations, Cambridge University Press, Cambridge, 1990.

[51] E.F. McClennen, Pragmatic rationality and rules, Philosophy and Public Affairs 26 (3) (1997) 210-258.

[52] T.D. Nielsen, J.-Y. Jaffray, An operational approach to rational decision making based on rank dependent utility, 2001, unpublished manuscript available on http://www.cs.aau.dk/ tdn/papers/nielsen-jaffray-01.pdf.

[53] T.D. Nielsen, F.V. Jensen, Welldefined decision scenarios, in: Proceedings of the Fifteenth Conference on Uncertainty in Artificial Intelligence, Morgan Kaufmann, Stockholm, Sweden, July 1999, pp. 502-511.

[54] J. Pearl, Probabilistic Reasoning in Intelligent Systems: Networks of Plausible Inference, Morgan Kaufmann Publishers, Los Altos, CA, 1988.

[55] J.C. Quiggin, A theory of anticipated utility, Journal of Economic Behavior \& Organization 3 (4) (December 1982 ) $323-343$.

[56] H. Raiffa, Decision Analysis: Introductory Lectures on Choices under Uncertainty, Addison-Wesley, Massachusetts, 1968.

[57] P.A. Samuelson, Consumption theory in terms of revealed preference, Econometrica 15 (1948) 243-253.

[58] J.K. Satia, R.E. Lave Jr., Markovian decision processes with uncertain transition probabilities, Operations Research 21 (3) (May-June 1973) 728-740.

[59] M.J. Schervish, T. Seidenfeld, J.B. Kadane, I. Levi, Extensions of expected utility theory and some limitations of pairwise comparisons, in: Proceedings of the 3rd International Symposium on Imprecise Probabilities and Their Applications, Lugano, Switzerland, July 2003, pp. 496-510.

[60] T. Seidenfeld, A contrast between two decision rules for use with (convex) sets of probabilities: $\Gamma$-Maximin versus E-Admissibility, Synthese 140 (1-2) (May 2004) 69-88.

[61] T. Seidenfeld, M.J. Schervish, J.B. Kadane, Decisions without ordering, in: W. Sieg (Ed.), Acting and Reflecting, Kluwer Academic Publishers, Dordrecht, 1990, pp. 143-170.

[62] T. Seidenfeld, M.J. Schervish, J.B. Kadane, A representation of partially ordered preferences, Annals of Statistics 23 (6) (December 1995 ) $2168-2217$.

[63] R. Shachter, Bayes-Ball: the rational pastime (for determining irrelevance and requisite information in belief networks and influence diagrams), in: Proceedings of the 14th Annual Conference on Uncertainty in Artificial Intelligence (UAI-98), Morgan Kaufmann, San Francisco, CA, 1998 , pp. 480-487.

[64] R. Shachter, Efficient value of information computation, in: Proceedings of the 15th Annual Conference on Uncertainty in Artificial Intelligence (UAI-99), Morgan Kaufmann, San Francisco, CA, 1999, pp. 594-601.

[65] H.D. Sherali, C.H. Tuncbilek, A global optimization algorithm for polynomial programming problems using a reformulation-linearization technique, Journal of Global Optimization 2 (1) (March 1992) 101-112.

[66] H.A. Simon, A behavioral model of rational choice, The Quarterly Journal of Economics 69 (1) (1955) 99-118.

[67] R. Strotz, Myopia and inconsistency in dynamic utility maximization, The Review of Economic Studies 23 (3) (1956) 165-180.

[68] J.A. Tatman, R.D. Shachter, Dynamic programming and influence diagrams, IEEE Transactions on Systems, Man and Cybernetics 20 (2) (1990) 365-379.

[69] F.W. Trevizan, F.G. Cozman, L.N. de Barros, Planning under risk and Knightian uncertainty, in: International Joint Conference on Artificial Intelligence, 2007, pp. 2023-2028.

[70] M.C.M. Troffaes, Decision making with imprecise probabilities: A short review, in: F. Cozman (Ed.), Society for Imprecise Probability Theory and Applications Newsletter, Manno, Switzerland, December 2004, pp. 4-7.

[71] M.C.M. Troffaes, Decision making under uncertainty using imprecise probabilities, International Journal of Approximate Reasoning 45 (1) (2007) 17-29.

[72] L.V. Utkin, T. Augustin, Powerful algorithms for decision making under partial prior information and general ambiguity attitudes, in: Proceedings of the 4th International Symposium on Imprecise Probabilities and Their Applications, Pittsburgh, Pennsylvania, July 2005 , pp. 349-358.

[73] P. Walley, Statistical Reasoning with Imprecise Probabilities, Chapman and Hall, London, 1991.

[74] C.C. White III, H.K. El-Deib, Markov decision processes with imprecise transition probabilities, Operations Research 42 (4) (July-August 1994 ) 739-749. 